\title{
CHARACTERIZATION OF RESISTANCE TO HALOGENATED AROMATIC HYDROCARBONS IN A POPULATION OF FUNDULUS BETEROCLITUS FROM A MARINE SUPERFUND SITE
}

\author{
By \\ Submitted in partial fulfillment of the requirements for the degree of
}

\begin{abstract}
Susan M. Bello
B.S., Michigan State University, 1993

Doctor of Philosophy

at the

MASSACHUSETTS INSTITUTE OF TECHNOLOGY

and the

WOODS HOLE OCEANOGRAPHIC INSTITUTION
\end{abstract}

September 1999
(C)1999 Susan Bello
All rights reserved.

The author hereby grants to MIT and WHOI permission to reproduce paper and electronic copies of this thesis in whole or in part and to distribute them publicly.

Signature of Author

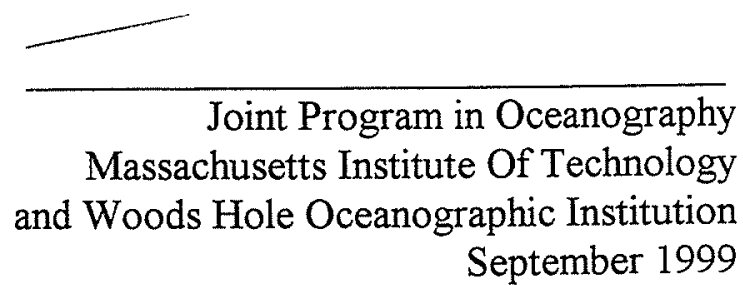

Certified by

Mark E. Hahn

Thesis Supervisor

Accepted by

Mark E. Hahn

Chair, Joint Committee for Biological Oceanography

Massachusetts Institute Of Technology/

Woods Hole Oceanographic Institution 


\title{
Characterization Of Resistance To Halogenated Aromatic Hydrocarbons In A Population Of Fundulus heteroclitus From A Marine Superfund Site
}

\author{
By \\ Susan M. Bello \\ Submitted to the Department of Biological Oceanography \\ September 3, 1999 in Partial Fulfillment of the Requirements \\ for the degree of Doctor of Philisophy in Biological Oceanography
}

\section{$\underline{\text { Abstract }}$}

New Bedford Harbor (NBH), MA, is contaminated with halogenated aromatic hydrocarbons (HAH) including some potent aryl hydrocarbon receptor (AhR) agonists. To determine if Fundulus heteroclitus from NBH have developed resistance to $\mathrm{HAH}$, we examined the inducibility of cytochrome P4501A1 (CYP1A1) in fish from NBH and Scorton Creek (SC, reference site). Despite higher PCB concentrations in NBH than in SC fish - 1500-fold CYP1A1 expression, in most tissues, was not higher in NBH fish than in $\mathrm{SC}$ fish. Glutathione S-transferase (GST) and UDP-glucuronosyltransferase (UGT) activities were higher in NBH fish than in SC fish, but only when fish were collected during different seasons. GST activity was higher in the intestines of NBH fish than in any other tissue.

2,3,7,8-Tetrachlorodibenzofuran (TCDF) induced CYP1A1 expression, in all tissues examined, in SC fish. In contrast, NBH fish showed little CYP1A1 induction by any measure, in any tissue. Hepatic GST activity was induced only in male NBH fish. Hepatic UGT activity showed no relationship to treatment in fish from either site.

2,3,7,8-Tetrachlorodibenzo-p-dioxin (TCDD) and $\beta$-naphthoflavone (BNF) induced CYP1A1 activity to the same level in primary cultures of hepatocytes from either SC or NBH fish. However, hepatocytes from NBH fish were 14-fold less sensitive to TCDD and 3-fold less sensitive to BNF than hepatocytes from SC fish.

To examine the heritability of resistance, $\mathrm{NBH}$ and $\mathrm{SC} \mathrm{F}_{1}$ fish were treated with ${ }^{3} \mathrm{H}$ TCDD or BNF. ${ }^{3} \mathrm{H}-\mathrm{TCDD}$ induced CYP1Al expression only in $\mathrm{SC} \mathrm{F}_{1}$ fish. BNF induced CYP1A1 expression in both $\mathrm{SC}$ and $\mathrm{NBH} \mathrm{F}_{1}$ fish. There was no significant difference in hepatic ${ }^{3} \mathrm{H}$-TCDD concentrations between $\mathrm{SC}$ and $\mathrm{NBH} \mathrm{F}_{1}$ fish.

Hepatic AhR content, as measured by photoaffinity labeling with ${ }^{125} \mathrm{I}_{-} \mathrm{N}_{3} \mathrm{Br}{ }_{2} \mathrm{DD}$, was lower in NBH fish than in SC fish and lower in males than in females. After 90 days in captivity, the sex difference persisted, but the site difference did not. TCDF induced hepatic AhR content in $\mathrm{NBH} \mathrm{F}_{1}$ fish.

These results indicate that NBH Fundulus have developed a pre-translational, systemic, heritable resistance to HAHs. These findings suggest that an alteration in the AhR pathway is responsible for this resistance; this is the subject of continuing research.

Thesis Supervisor: Mark E. Hahn

Title: Associate Scientist 


\section{Acknowledgments}

First, I have to thank my parents and my sister for their love, support, and understanding; without them I would probably not have finished this work.

Next, I owe more than I can say to my thesis advisor and friend Mark Hahn. He was always available whenever I needed to consult on ideas, was endlessly tolerant of the bizarre hours I kept, and fostered a truly enjoyable work environment.

Bruce Woodin was an ever accommodating and delightful person to work with. I want to thank him for all of his help with dissections, his enlightening field trips, and for generally making my time here that much more enjoyable. I thank Diana Franks for her work with the primary culturing of the Fundulus hepatocytes, for her help with fish care and for generally helping to make my life a little easier. Diane Nacci, Ken Rocha, and the EPA laboratory in Narragansette for kindly providing fish. Diane also for her help during the planning stages of this work and her willingness to exchange results. Adria Elskus for the PCB analysis and her advice.

I need to thank all of my labmates Connie Hart, Eli Hesterman, Brenda Jensen, Sibel Karchner, and Wade Powell for their friendship and for their help. I would also like to thank Shannon Bard and Joanna Wilson, their willingness to listen when I needed to vent made my time here much less stressful. Jennifer Schlezinger and Rachel Cox, my officemates, for their tolerance and advice.

My thanks also go to Kathy Barbeau, Liz Minor, Lisa Max, Tad Snow, Jay Austin and Hobbes, the full and part time residents of Castle Angst, a better group of housemates I could not have found no matter how I tried.

I thank my committee John Stegeman, Judy McDowell, Keith Cooper, and Phil Gschwend for their advice and comments. They were essential in guiding this work.

I thank the Joint Committee for Biological Oceanography and the Education Office for their support.

This work was supported by a National Science Foundation Graduate Fellowship Program and by a National Institute of Environmental Health and Safety grant P42 ES07381 (Superfund Basic Research Program at Boston University) 


\section{Table of Contents}

Abstract

Acknowledgments

Table of Contents

List of Figures

List of Tables

Abbreviations Used

Chapter 1. Introduction

Introduction

Background Material

New Bedford Harbor

Fundulus heteroclitus Biology

Resistance

Resistance and Fundulus heteroclitus

Dioxin Toxicity

Dioxin and AhR

AhR Signal Transduction Pathway

AhR in Mammals

AhR in Fish

AhR Influenced Genes

ARNT 
$\begin{array}{ll}\text { ARNT in Fish } & 38\end{array}$

$\begin{array}{ll}\text { DRE } & 38\end{array}$

Cytochrome P450 1A1 39

Glutathione S-Transferase $\quad 42$

$\begin{array}{ll}\text { UDP-Glucuronosyltransferase } & 42\end{array}$

$\begin{array}{ll}\text { Specific Aims } & 43\end{array}$

Chapter 2: EXPRESSION OF XENOBIOTIC METABOLIZING ENZYMES AND 45

CHARACTERIZATION OF PCB CONCENTRATIONS IN AN ESTUARINE

TELEOST (FUNDULUS HETEROCLITUS) FROM THE NEW BEDFORD

HARBOR, MA SUPERFUND SITE.

$\begin{array}{ll}\text { Introduction } & 46\end{array}$

$\begin{array}{ll}\text { Materials and Methods } & 48\end{array}$

$\begin{array}{lr}\text { Materials } & 48\end{array}$

Fundulus Collection and Maintenance $\quad 48$

$\begin{array}{lr}\text { Tissue Collections } & 48\end{array}$

Microsomal Preparation $\quad 49$

Enzyme Activity Assays $\quad 50$

Protein and RNA Blotting 51

$\begin{array}{ll}\mathrm{IHC} & 52\end{array}$

PCB Analysis $\quad 52$

Results

Characteristics of SC and NBH Fundulus 
$\begin{array}{ll}\text { CYP1A1 Activity and Expression } & 70\end{array}$

$\begin{array}{lr}\text { GST Activity } & 80\end{array}$

$\begin{array}{ll}\text { UGT Activity } & 80\end{array}$

$\begin{array}{ll}\text { Discussion } & 86\end{array}$

Chapter 3: RESISTANCE TO HALOGENATED AROMATIC HYDROCARBONS 93

IN A CHRONICALLY EXPOSED POPULATION OF KILLIFISH: IN VIVO

AND IN VITRO STUDIES ON THE INDUCTION OF XENOBIOTIC

METABOLIZING ENZYMES IN FERAL FISH.

Introduction

Materials and Methods

Materials

Fundulus Collection and Maintenance

Fundulus Treatments

Hepatocyte Culture

Microsome and Cytosol Preparation

Enzyme Activity Assays

Protein and RNA Blotting

IHC

Results

In Vivo Studies 
Characteristics of SC and NBH Fundulus 103

$\begin{array}{ll}\text { CYP1A1 Activity and Expression } & 105\end{array}$

$\begin{array}{ll}\text { GST Activity } & 122\end{array}$

$\begin{array}{ll}\text { UGT Activity } & 122\end{array}$

$\begin{array}{lr}\text { In Vitro Studies: CYP1A1 Activity } & 122\end{array}$

$\begin{array}{ll}\text { Discussion } & 127\end{array}$

Chapter 4: HERITABILITY OF DIOXIN RESISTANCE IN FUNDULUS 133

HETEROCLITUS FROM NEW BEDFORD HARBOR, MA.

Introduction

$\begin{array}{ll}\text { Materials and Methods } & 136\end{array}$

$\begin{array}{ll}\text { Materials } & 136\end{array}$

$\begin{array}{ll}\text { Fundulus Collection and Maintenance } & 139\end{array}$

$\begin{array}{ll}\text { Experiment 1: } & 136\end{array}$

Fundulus Treatments $\quad 136$

Microsome and Cytosol Preparation 137

$\begin{array}{ll}\text { Enzyme Activity Assays } & 137\end{array}$

$\begin{array}{ll}\text { Protein and RNA Blotting } & 138\end{array}$

$\begin{array}{ll}\text { IHC } & 139\end{array}$

$\begin{array}{ll}\text { PCB Analysis } & 140\end{array}$

$\begin{array}{ll}\text { Experiment } 2 & 140\end{array}$

$\begin{array}{ll}\text { Fundulus Treatments } & 140\end{array}$

Homogenate Preparation 141 
Results

Experiment 1

Characteristics of NBH Fundulus

CYP1A1 Activity and Expression

GST and UGT Activity

PCB Analysis

Experiment 2

Characteristics of $\mathrm{SC}$ and $\mathrm{NBH} \mathrm{F}_{1}$ Fish

CYP1A1 Activity and Expression

TCDD Distribution

160

Discussion ASSAYS.

Introduction

Materials and Methods 
Catalase Labeling

Sucrose Gradients

Photoaffinity Labeling

172

Fundulus AhR1 and AhR2 Synthesis

173

Results

174

Sucrose Gradients

174

Photoaffinity Labeling

181

Discussion

186

Chapter 6: SUMMARY, CONCLUSIONS, AND SPECULATIONS

189

Resistance to HAH in Fundulus heteroclitus from NBH

190

Future Directions

193

Speculations on Ramifications

194

Bibliography

196 


\section{List of Figures}

\section{Chapter 1:}

1-1: Map of Collection Sites

1-2: Chemical Structures AhR Agonists 29

1-3: Signal Transduction Pathway 33

1-4: PAS Protein Structure $\quad 34$

1-5: CYP1A1 Metabolism $\quad 41$

Chapter 2:

2-1: Distribution of PCBs in Carcasses (A) and Livers (B) 65

2-2: Distribution of PCB Isomers in SC and NBH Fish 67

2-3: EROD Activity in Liver Extracts 69

2-4: EROD Activity (A) and CYP1A1 (B) Protein Levels During the First 90 Days After Collection

2-5: IHC analysis of CYP1A1 in the Atria and Kidneys of Fundulus from SC and NBH One Day After Collection (photographs).

2-6: CYP1A1 mRNA Levels in Fundulus from SC and NBH One Day After Collection.

2-7: GST (A) and UGT (B) Activity During the First 90 Days After Collection.

2-8: GST (A) and UGT (B) Activity in Hepatic and Extrahepatic Tissues of

Fundulus from SC and NBH One Day After Collection.

\section{Chapter 3:}

3-1: EROD Activity (A) and CYP1A1 (B) Protein Levels in Female Fundulus from SC and NBH treated with TCDF.

3-2: IHC analysis of CYP1A1 in the Atria of Female Fundulus from SC and NBH treated with TCDF (photographs).

3-3: IHC analysis of CYP1A1 in the Heart, Spleen, and Gonad of Female Fundulus from $\mathrm{SC}$ and $\mathrm{NBH}$ treated with TCDF.

3-4: CYP1A1 mRNA Levels in Male Fundulus from SC and NBH treated with 
TCDF.

3-5: IHC analysis of CYP1A1 in the Heart, Kidney, Intestine, and Gill of Male

Fundulus from SC and NBH treated with TCDF

3-6: GST Activity in Female (A) and Male (B) Fundulus from SC and NBH

Treated with TCDF.

3-7: UGT Activity in Female (A) and Male (B) Fundulus from SC and NBH

Treated with TCDF.

3-8: CYP1A1 Activity in Hepatocytes from SC or NBH Fundulus Treated with TCDD or BNF.

Chapter 4:

4-1: Distribution of PCBs in Carcasses of $F_{1}$ Fish. 146

4-3: Comparison of PCB \#138 Ratios of Selected Congeners 149

4-2: EROD Activity as a Function of Measured Liver ${ }^{3} \mathrm{H}$-TCDD Concentrations. 155

4-3: IHC analysis of CYP1A1 in the Atria of $\mathrm{SC}$ and $\mathrm{NBHF}_{1}$ Fish Treated with 157

TCDD or BNF (photographs)

4-4: IHC analysis of CYP1A1 in the Atria and Kidney of SC and $\mathrm{NBH}_{1}$ Fish

Treated with TCDD or BNF

Chapter 5:

5-1: Sucrose Density Gradient Analysis of Hepatic Cytosolic AhR Content in

Fundulus from SC, One Day After Collection.

5-2: Sucrose Density Gradient Analysis of Hepatic Cytosolic AhR Content in

Fundulus from NBH, One Day After Collection.

5-3: SDS-PAGE Analysis of Hepatic Cytosols from SC and NBH Fundulus

5-4: Photoaffinity Analysis of Hepatic Cytosolic AhR Content in Fundulus from $\mathrm{SC}$ and NBH, One Day After Collection.

5-5: Photoaffinity Analysis of Hepatic Cytosolic AhR Content in Fundulus from SC and NBH, One and 90-Days After Collection, and Control and TCDF Treated $\mathrm{NBH} \mathrm{F}_{1}$ Fish 


\section{List of Tables}

Chapter 2:

2-1: Sample Collections

2-2: Characteristics of Fundulus from SC and NBH

2-3: PCB Congener Concentrations in the Carcasses of Fundulus from SC 58 and $\mathrm{NBH}$

2-4: PCB Congener Concentrations in the Livers of Fundulus from SC and $\mathrm{NBH}$

2-5: EROD Activity in Hepatic and Extrahepatic Tissues of Fundulus from

$\mathrm{SC}$ and $\mathrm{NBH}$

2-6: CYP1A1 Protein in Extrahepatic Tissues of Fundulus from

$\mathrm{SC}$ and $\mathrm{NBH}$

\section{Chapter 3:}

3-1: Characteristics of Fundulus from SC and NBH Treated with TCDF (Experiment 1)

3-2: Characteristics of Fundulus from SC and NBH Treated with TCDF (Experiment 2)

3-3: Induction of Xenobiotic Metabolizing Enzymes in Male Fundulus from SC and NBH Treated with TCDF

3-4: EROD Activity in Hepatocyte Cultures from the livers of SC and NBH Fundulus Treated with TCDD or BNF 
Chapter 4:

4-1: Characteristics of and Induction of Xenobiotic Metabolizing Enzymes in NBH $F_{1}$ Fish Treated with TCDF

4-2: IHC analysis of CYP1A1 in Hepatic and Extrahepatic Tissues of NBH F 1

Fish Treated with TCDF

4-3: Characteristics of SC and $\mathrm{NBH} \mathrm{F}_{1}$ Fish Treated with TCDD and BNF

4-4: Hepatic ${ }^{3} \mathrm{H}-\mathrm{TCDD}$ Concentrations and EROD Activity in SC and NBH

$\mathrm{F}_{1}$ Fish Treated with TCDD

4-5: Extrahepatic ${ }^{3} \mathrm{H}-\mathrm{TCDD}$ Concentrations in $\mathrm{SC}$ and NBH $\mathrm{F}_{1}$ Fish Treated with TCDD

\section{Chapter 5:}

5-1: AhR Content in Fundulus from SC and NBH, Sucrose Density Gradient Analysis 
NBH: New Bedford Harbor

PCB: polychlorinated biphenyls

PCDF: polychlorinated dibenzofurans

PCDD: polychlorinated dibenzodioxins

CYP1A1: Cytochrome P450 1A1

SC: Scorton Creek

AhR: Aryl hydrocarbon receptor

ARNT: AhR nuclear translocator

HSP90: Heat shock protein 90

DRE: Dioxin responsive element

DLC: dioxin-like-compounds

HAH: Halogenated aromatic hydrocarbon

PAH: Polycyclic aromatic hydrocarbon

TCDF: 2,3,7,8-tetrachlorodibenzofuran

TCDD: 2,3,7,8-tetrachlorodibenzo-p-dioxin

BNF: $\beta$-naphthoflavone

CDNB: 1-chloro-2,4-dinitrobenzene

EROD: ethoxyresorufin O-deethylase

GST: Glutathione S-transferase 
UGT: Uridine Diphosphate-Glucuronosyl Transferase

LSI: Liver / Somatic Index

GSI: Gonad / Somatic Index

MAb: monoclonal antibody

TBS: tris buffered saline

IHC: Immunohistochemistry

PBS/BSA: bovine serum albumin in phosphate buffered saline ANOVA: Analysis of Variance 
Chapter 1: INTRODUCTION

$\begin{array}{ll}\text { Introduction } & 18\end{array}$

Background Material 20

New Bedford Harbor $\quad 20$

Fundulus heteroclitus Biology

$\begin{array}{ll}\text { Resistance } & 25\end{array}$

Resistance and Fundulus heteroclitus $\quad 26$

Dioxin Toxicity 28

Dioxin and AhR 31

AhR Signal Transduction Pathway 31

AhR in Mammals $\quad 35$

AhR in Fish $\quad 36$

AhR Influenced Genes $\quad 37$

$\begin{array}{ll}\text { ARNT } & 37\end{array}$

$\begin{array}{ll}\text { ARNT in Fish } & 38\end{array}$

$\begin{array}{ll}\text { DRE } & 38\end{array}$

Cytochrome P450 1Al 39

$\begin{array}{ll}\text { Glutathione S-Transferase } & 42\end{array}$

UDP-Glucuronosyltransferase $\quad 42$

Specific Aims 


\section{Introduction}

The long-term effects of contaminants in marine organisms are poorly understood. Most studies consider only the effects of acute exposures and those few studies that do attempt to examine chronic exposures rarely examine more than one or two generations. The results of these studies are then used to determine which biological or biochemical effects should be monitored in wild-populations to assess exposure to environmental contaminants. An implicit assumption in this approach is that the range of responses in a population will remain constant over multiple generations. A few studies have cast doubt on this assumption, indicating that exposure to high concentrations of some pollutants including halogenated aromatic hydrocarbons $(\mathrm{HAH})$, polycyclic aromatic hydrocarbons $(\mathrm{PAH})$, and heavy metals, can change responses in some populations (reviewed in (Hahn, 1998)).

Natural populations are comprised of individuals that often vary in their sensitivity to toxic compounds. Long-term exposures to these compounds can exert selective pressure on such populations. One possible result is the development of resistance to the compound or class of compounds. The classic examples of this phenomenon are resistance to antibiotics in bacteria and to insecticides in insects.

An understanding of the phenomenon of acquired resistance is important for better understanding of the ecological impacts of pollution, more accurate interpretation of biomonitoring programs, development of appropriate biomarkers for highly contaminated sites, and the prediction of impacts of additional stressors or remediation efforts. Acquired resistance will change the range of responses in a population to the compound or class of compounds against which the resistance has developed. This could result in false negative results in biomonitoring studies, if the response of the biomarker has been altered. It may be possible to use acquired resistance itself as a biomarker for highly impacted sites; as this phenomenon indicates that the pollutant has effected not just individual organisms but the population as well. Since acquired resistance is the result of directed selective pressure, it will necessarily change the gene pool in the affected population; most likely resulting in a decrease in genetic diversity in the affected population. This decrease in diversity may negatively affect the ability of individuals or the whole population to adapt to additional stressors. Finally, resistant individuals, by definition, can tolerate higher concentrations of a chemical than can sensitive 
individuals. If the compound to which resistance has been developed happens to bioaccumulate, the high body burdens in resistant organisms may constitute a significant reservoir of the compound, complicating remediation efforts.

In this thesis, I examined a population of fish, Fundulus heteroclitus (killifish), from the New Bedford Harbor (NBH), MA, Superfund site to determine if this population had developed resistance to HAHs. NBH is heavily contaminated with polychlorinated biphenyls (PCBs) and to a lesser extent with dibenzofurans and dibenzo-p-dioxins (Weaver, 1984). These compounds were released over the course of $\sim 3$ decades, beginning in the 1940's. The generation time of killifish is $\sim 1.5$ years (Able, 1984); suggesting that if the contaminants in NBH had any selective effect on the killifish population there had been ample time for resistance to develop.

The classical biomarker of exposure to HAHs is the induction of cytochrome P450 1A1 (CYP1A1) (Stegeman et al., 1992). Induction of CYP1A1 is mediated by interaction of the inducer with the aryl hydrocarbon receptor (AhR) signal transduction pathway (Safe and Krishnan, 1995). CYP1A1 in killifish have been shown to be inducible both by laboratory (Kloepper- Sams and Stegeman, 1989; Prince, 1995b) and environmental (Burns, 1976; Elskus, 1989) exposures to HAHs and PAHs. The components of the AhR signal transduction pathway have also been identified in killifish (Hahn et al., 1997; Morrison et al., 1998;

Karchner et al., 1999; Powell et al., 1999), making killifish an ideal species in which to examine acquired resistance to HAHs. 


\section{Background}

\section{New Bedford Harbor}

New Bedford Harbor (NBH) is a Superfund site in eastern Massachusetts (Fig. 1-1) The main source of contamination in the upper harbor was the release of PCBs used in the manufacturing of capacitors. (PCBs in the USA were manufactured solely by the Monsanto corporation. PCBs were produced as complex mixtures of the various congeners ( 209 in all). The mixtures were referred to as Aroclors, and named according to the percent of chlorination.) In $\mathrm{NBH}$, the Aroclors released consisted mostly of 1242 (42\% chlorinated) and 1254 (54\% chlorinated). Capacitor manufacturing began around 1947 and continued until the late 70's (Lake, 1995). During this time, the harbor was also contaminated with dibenzofurans, dibenzo-p-dioxins, and heavy metals, but the PCBs are the major contaminant (Pruell et al., 1990 ) in the Superfund site. The contamination was first discovered in 1976 during an EPA PCB survey of New England. Elevated concentrations have been found throughout the entire harbor and well out into Buzzards Bay. Levels in the sediment ranged from a few ppm to well over $100,000 \mathrm{ppm}$ (Weaver, 1984). In contrast, the highest reported PCB level in any other New England harbor was only 17.1 ppm (Pruell et al., 1990). In the water column, levels exceeded the EPA's limit (0.001 ppb) by over 1000 fold (Weaver, 1984). NBH was added to the EPA Superfund list in the early 1980's.

Following the listing, most of the harbor was closed to fishing. The harbor was divided into three sections, loosely corresponding to the inner, middle, and outer harbor. The Superfund site is limited to the area of the harbor above the 195 bridge (Fig. 1-1). The extent of the fishing ban varied in each section, with the inner harbor being closed for all takings of finfish, shellfish and lobsters, the middle harbor allowing the taking of non-bottom feeding finfish and shellfish, and the outer harbor only closed to lobster fishing. Local fishermen have sued the state and the manufacturers over these closings, since the outer harbor area includes the lobstering grounds in Buzzards Bay. Lobsters in the outer harbor were extensively surveyed in 1982; mean PCB concentrations were found to be $4.4 \mathrm{ppm}$ with a high of $8.8 \mathrm{ppm}$ and a low of $0.7 \mathrm{ppm}$ (Weaver, 1984). At the time, this level was just below the FDA action limit of $5.0 \mathrm{ppm}$ in the edible portion of shellfish; this limit has since been lowered to $2 \mathrm{ppm}$. 
Measurements of water column PCB concentrations taken in 1991, 1992, and 1993 $(1.5,3.2$, and $3.4 \mathrm{ppb}$ respectively) continued to exceed the EPA guideline both in the hotspot (the area closest to the source of contamination, which has the highest reported PCB levels, Fig. 1-1) as well as near the lower end of the Superfund site (a mean $1.87 \mathrm{ppb}$ was found at Coggeshall bridge over the same 3 years) (Lake, 1995). Some of these recent elevated water column levels may be the result resuspension of contaminated sediments during the dredging of the most contaminated sediments. Levels in fish and shellfish from the area greatly exceed the FDA guidelines of $2 \mathrm{ppm}$. F. heteroclitus from the hot spot and Coggeshall bridge area had mean body burdens over these 3 years of $1370 \mathrm{ppm}$ and $655 \mathrm{ppm}$ respectively. Mussels from these 2 sites had mean PCB concentrations of $732 \mathrm{ppm}$ and $128 \mathrm{ppm}$. Eels from the same study collected just south of the hot spot had mean PCB concentrations of 380 ppm (Lake, 1995). All of these measurements were made before the dredging project had finished and represent a cumulative exposure to contaminated sediments. The results of the remediation may not be seen for some time, but the accumulated data may allow any recovery to be readily discerned. 
Fig. 1-1: Map of collection sites. SC (Scorton Creek) reference site; NBH (New Bedford Harbor) contaminated site. Inset NBH showing the hotspot and manufacturing plant that was a major PCB source 


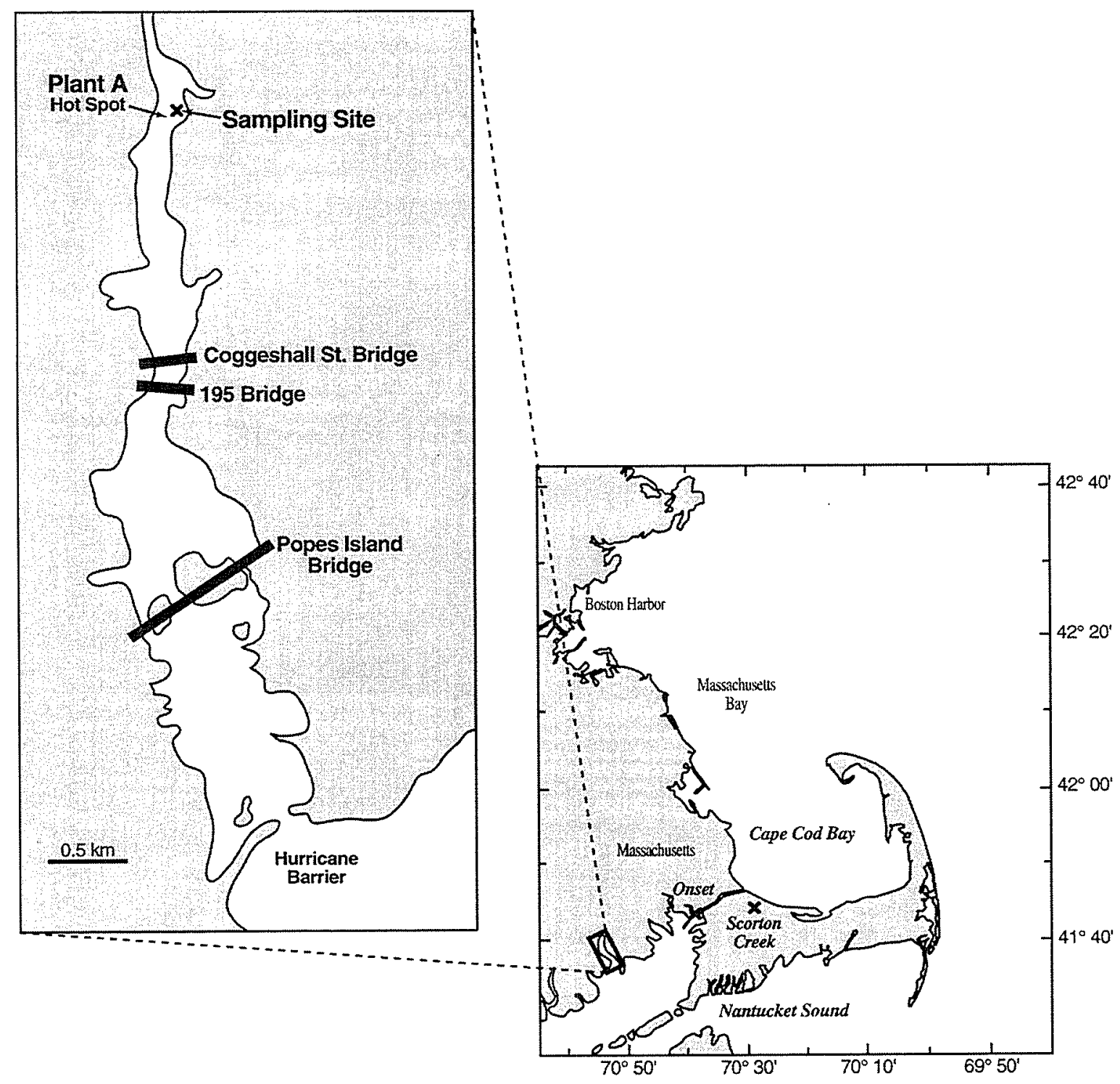


A number of studies have been conducted to assess the biological impacts of this contamination using a variety of organisms. Soft shell clams (Mya arenaria) from NBH had a consistently elevated prevalence of leukemia (Harper et al., 1994). This disease state could be induced in transplanted clams in as little as 6 months (Craig et al., 1993). These studies were conducted in the 'mid-harbor' (below the 195 bridge) away from the areas of highest PCB contamination. Studies in winter flounder (Pseudopleuronectes americanus) found that in the laboratory larvae from these fish are smaller in length and weight than larvae from uncontaminated fish (Black et al., 1988). Mean PCB concentrations in the liver of adult winter flounder was $155 \mathrm{ppm}$. CYP1Al protein expression was elevated in these fish, compared to reference fish, although ethoxyresorufin O-deethylase (EROD; a measure of CYP1Al activity, see below) activity was not (Elskus et al., 1989). Scup (Stenotomus chrysops) collected in the outer harbor (outside the hurricane barrier) also had elevated expression of CYP1A1 protein and EROD activity (Stegeman et al., 1991). Smooth dogfish (Mustelus canis) collected just outside NBH had elevated levels of CYP1A1 compared to animals collected in Vineyard Sound (Hahn et al., 1998). Finally, eels (Anguilla rostrata) collected at the 'hot spot' also had elevated EROD activity (Schlezinger and Stegeman, 1999).

\section{Fundulus heteroclitus biology}

There are a few key facts of killifish, Fundulus heteroclitus, biology and life history that are important to this study. First, killifish do not have large home ranges (Lotrich, 1975) and are not believed to frequently migrate to new areas (Powers et al., 1986). The little migratory behavior that has been reported was migration during the winter months towards lower salinity water (Fritz et al., 1975). Second, their eggs are surrounded by a sticky chorion and spawning usually occurs in salt marsh grass and muscle beds (Taylor, 1986), therefore Fundulus eggs are not typically dispersed far from the spawning site. These factors may act to isolate the NBH population from other populations in uncontaminated sites. The existence of a hurricane barrier across the mouth of NBH may also decrease migration into the harbor. All of the organisms used in the studies mentioned in the NBH section (above) have at least one stage in their lifecycle during which dispersal to remote sites typically occurs. The influx of individuals from uncontaminated sites might interfere with the development of resistance in the local population. 
This type of interference should not occur in the development of resistance in a killifish population. Finally the short generation time of killifish (offspring can reach sexual maturity in one year (Able and Hata, 1984)) could allow for noticeable changes in population characteristics to occur in only a few decades.

Killifish may burrow into the sediments during the winter (Chidester, 1920). This behavior could help them survive the cold weather while in shallow water, but would also increase their contact with contaminated sediments. Killifish are opportunistic feeders eating a wide range of plant and animal material. These behaviors may tend to increase the amount of PCBs that killifish accumulate. The PCB body burdens reported in NBH killifish are extremely high (1370 ppm, compared to the FDA action guideline of $2 \mathrm{ppm}$ ) suggesting that the killifish could have significant negative effects on their predators.

\section{Resistance}

Resistance mechanisms can be separated into two broad categories, active and passive. Active resistance requires the continual input of energy to prevent damage. Mechanisms that can be included in this category include the p-glycoprotein based multi-xenobiotic resistance and metallothioneins. Both of these mechanisms result in lower concentrations of the compound at the active site. The multi-xenobiotic (or drug) resistance (MXR) requires the constant input of ATP to pump chemicals, which have diffused in through the cytoplasmic membrane, out of the cell against a gradient (reviewed in (Endicott and Ling, 1989)). This mechanism can be overwhelmed by a large enough dose, can cost the organism a great deal of energy to maintain, and may be futile if the chemical is slowly metabolized or difficult to excrete and continues to diffuse back into the cell. In the present study, the MXR mechanism could play a role. TCDD has been reported to induce p-glycoprotein expression in some animals (Burt and Thorgeirsson, 1988; Schuetz et al., 1995). If some tissues are relatively more sensitive than other tissues, than overexpression of the p-glycoprotein in these tissues could protect the organism. However, the extremely high body burdens of PCBs in NBH killifish suggest that such a mechanism would be overwhelmed.

Metallothioneins (MT), 6 to $7 \mathrm{kDa}$, cysteine rich metal binding proteins, are induced in response to exposure to metals. These proteins typically bind to metals such as zinc, cadmium, 
and mercury and may be involved in the increase in tolerance for metals seen after exposure to low levels of a metal (Klerks and Weis, 1987). Studies in metallothionein-null mice have shown that these proteins protect against the toxicity of some metals (Satoh et al., 1997; Liu et al., 1998). Metallothioneins also have a role in maintaining homeostasis of essential metals, such as zinc and copper (Roesijadi, 1992; Kelly et al., 1996). Again the protective role requires energy to increase production of the proteins and then maintain an effective concentration of unbound protein. This mechanism can also be overwhelmed by a high enough dose or prolonged exposure. Metalothioniens are unlikely to play any role in resistance to the HAHs, but theoretically a $\mathrm{HAH}$ binding protein, with no receptor function, could function in a similar manner to protect against HAH toxicity. A possible example of this is type of a protein is CYP1A2, which has been reported to bind up to $90 \%$ of the total TCDD in mice (Diliberto et al., 1999). Active resistance can stress an organism; making it less capable of successfully meeting additional challenges.

Passive resistance can also be overwhelmed by a sufficiently high dose but does not cost the organism any energy while it is functioning. These animals simply are not as sensitive to the toxicant as other organisms. Examples of this type of resistance are the Ah-nonresponsive mouse strains. These strains can be exposed to levels of $\mathrm{HAH} 10$ to 15 fold higher than sensitive mice before eliciting a response in the resistant mouse (Okey et al., 1989). There may be other costs associated with the tolerant phenotype such as impaired immune function (as seen in $\mathrm{AhR}^{-}$mice and, under chronic exposure conditions, in low affinity AhR mice) (Fernandez Salguero et al., 1995) (Morris and Holsapple, 1991), which are less tangible and difficult to quantify. It is unknown which strategy or combination of strategies, active and/or passive, is occurring in the NBH fish.

\section{Resistance and Fundulus heteroclitus}

The occurrence of decreased chemical sensitivity in Fundulus heteroclitus living in polluted environments (when compared to killifish from clean environments) has been documented several times. The phenotype of resistance differs in each case, presumably depending on the types and concentrations of the pollutants in each site. Killifish from a creosote polluted site (containing PAHs) in VA had embryos that were resistant to acute 
exposures, but the adults were susceptible to sublethal effects of long term exposures. This susceptibility was manifested as a high prevalence of hepatic lesions in wild caught fish (Vogelbein et al., 1990) and in the abnormal function of cytotoxic cells (Faisal et al., 1991). These fish have elevated expression of glutathione S-transferase in the liver and intestine (Armknecht et al., 1998), which may protect them from some of the toxic effects of the PAHs. CYP1A1 expression was seasonally elevated in these fish, compared to fish from a reference site, but was not induced by exposure to 3-mthylcholanthrene (Van Veld and Westbrook, 1995).

Another population of resistant killifish was found in Newark Bay, NJ (Prince, 1995a) The major pollutant in this bay is TCDD. Again, embryos show resistance to acutely lethal exposures, and the adults appear to be susceptible to long term exposures, as shown by increased prevalence of hepatic lesions. Adults exhibit consistently elevated hepatic CYP1A1 activities, increased liver to body weight ratios, and a loss of inducibility of CYP1A1 activity by 2,3,7,8-TCDD (Prince, 1995b). There appeared to be no change in TCDD metabolism or excretion rates despite the alterations in CYP1A1 activity.

F. heteroclitus from Piles Creek (NJ) have been shown to have developed resistance to some toxic metals (Weis and Weis, 1989). This site is contaminated with mercury and lead from surrounding industrial sources. This resistance appears to be restricted to particular developmental stages (embryo and larvae) of the fish and a form of mercury (meHg). In unexposed populations the sensitivity of egg batches laid by different females, as determined by percentage of eggs that failed to hatch at any given dose of meHg, varies greatly. In the exposed population the range of sensitivities is greatly reduced (Weis et al., 1981). Reduced sensitivity was accompanied, in both populations, by a number of other traits including higher numbers of fin rays in females and shorter, sparser chorionic fibers. Short term selection pressure can result in a rapid shift of the population towards the less-sensitive extreme. This change can occur in one spawning season. But without continued pressure the population can return to the prior heterogeneous state (Weis and Weis, 1989).

The tolerance to methyl-mercury (meHg) expressed by Fundulus is restricted to gametes and embryos, probably relating to the critical period for $\mathrm{meHg}$ toxicity, gastrulation, which occurs during the second day after fertilization. Because the critical period is so early in 
development the mechanism should not involve MTs, which are not expressed until several stages later in development. The PC embryos showed no decreased sensitivity to PAHs and actually seemed more sensitive to inorganic mercury than reference embryos (Weis and Weis, 1982). This increased sensitivity would seem to indicate that the Hg in PC is mostly meHg and that there is some trade off in terms of sensitivity to other pollutants that was made in the development of the PC tolerant phenotype.

\section{Dioxin (and Dioxin like) Toxicity}

Halogenated aromatic hydrocarbons ( $\mathrm{HAH}$ ), a class of compounds which includes polychlorinated-biphenyls (PCBs), dibenzofurans, and dibenzo-p-dioxins (Fig 1-2), are widespread contaminants in the environment. HAHs were produced by industries, either intentionally or as contaminants, before their toxic properties were fully understood; often they were simply disposed of by being dumped in a nearby body of water. To further complicate the problem these compounds, especially $\mathrm{PCBs}$, were often used in complex mixtures where little was known about what congeners were present in the mix. The degree of chlorination of a congener as well as its ability to achieve a planar or coplanar conformation influences the toxicity, hydrophobicity, and persistence of the congener. In general congeners with a planar or coplanar conformation (the mono- or non-ortho substituted compounds) are more toxic than congeners that are not planar (Okey, 1994). In mixtures, congeners may interact in an additive, synergistic, or antagonistic fashion. These mixtures constitute a significant threat to organisms, but their variable nature makes it difficult to judge the severity of the risk or the impact of past exposures.

For the purpose of this work dibenzo-p-dioxin (dioxin) will be used as a representative $\mathrm{HAH}$. The reasons for this are many. First when comparing the relative potency of different $\mathrm{HAH}$, dioxin, specifically 2,3,7,8-tetrachlorodibenzo-p-dioxin (TCDD, the most potent HAH), is used as a reference base line and potencies are reported as TCDD equivalents. Second, the slow degradation of many dioxins avoids complications created by metabolites of differing 


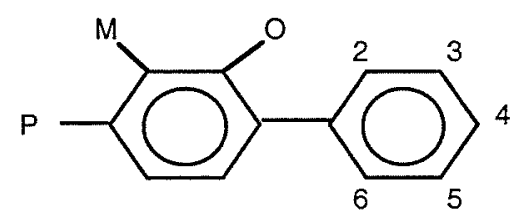

Polychlorinated biphenyls (209 congeners)<smiles>Clc1cc2c(cc1Cl)Oc1cc(Cl)c(Cl)cc1O2</smiles>

Chlorinated Dibenzodioxins (75 congeners)<smiles>Clc1cc2oc3cc(Cl)c(Cl)cc3c2cc1Cl</smiles>

Chlorinated Dibenzofurans (135 congeners)

Fig. 1-2: Chemical structure of $\operatorname{PCBs}(\mathrm{A})$, dibenzo-p-dioxins (B), dibenzofurans (C). The numbering and para, ortho, and meta positions are labeled on the PCBs. 2,3,7,8tetrachlorodibenzo-p-dioxin and 2,3,7,8-tetrachlorodibenzofuran are shown. 
potencies. Also the slow degradation and excretion prolongs the peak of induction as well as the life-span of receptor-ligand complexes. Third, many of the effects exerted by dioxins are also exerted by other HAH (Whitlock, 1990). Finally, virtually all of the effects of the planar and coplanar HAHs, which include TCDD, are dependent on the AhR signal transduction pathway (see below) (Fernandez Salguero et al., 1996).

Dioxins elicit a wide range of responses in an exposed organism. These responses have been examined across the spectrum of biological organization, from the whole organism through the tissues to molecular interactions. These responses include: the induction of CYP1Al, teratogenicity, thymic atrophy, hepatomegaly, and immunotoxicity (Holsapple et al., 1991; Safe and Krishnan, 1995; Mimura et al., 1997). Organismal effects are highly variable across species. One common tie is that an acutely lethal dose does not result in a rapid death, but instead a slow wasting with death occurring 1 to 2 weeks after administration of the dose. The exact cause of death from dioxin toxicity is not yet known (Poland and Knutson, 1982), but the resistance of AhR knock-out mice to this syndrome suggests that the AhR pathway is involved (Fernandez Salguero et al., 1996). Following a non-lethal exposure a number of organs are affected. These effects can be grouped into three general classes. Hyperplasia and/or altered cellular differentiation can be seen in the liver especially in the parenchymal cells, in the epidermis of some mammals, in the gastro-intestinal tract, in the epithelium, and the gills of fish. Tissue loss, hypoplasia, and/or atrophy can be seen throughout the lymphoid system, in the bone marrow, in the gastro-intestinal tract, and in the testes. Finally, there is a miscellaneous grouping that includes edema (Poland and Knutson, 1982).

Some of these cellular and tissue level changes can be related to the biochemical effects of dioxins. The most important of these, for the purpose of this thesis, are those which result from the binding to the AhR. Endocrine effects are believed to be altered by dioxin through the induction of some protein(s), most likely tumor necrosis factor (TNF-a), which is also a ligand for the estrogen receptor (Clark et al., 1991). There is also evidence that dioxin can alter levels of endocrine receptors, such as the progesterone and oestrogen receptors (Landers and Bunce, 1991). It is further probable that dioxin induces an enzyme, most likely a cytochrome P450, which can metabolize steroids. Alteration in steroid homeostasis may explain decreased sperm 
production and other changes in gonadal structure and function leading to decreased reproductive success in chronically exposed animals (Poland and Knutson, 1982).

\section{Dioxin and AhR}

A strong case can be made for the involvement of the AhR pathway in the toxicity of dioxins. Studies in AhR knock-out mice demonstrating greatly reduced susceptibility to the effects of TCDD (Fernandez Salguero et al., 1996) provide compelling evidence for the dominant role of AhR in the mechanism of TCDD toxicity. Comparison of the structureactivity relationships (SAR) for CYP1A1 induction and toxicity provides evidence that the results in mice can be extended to other species. In both of these SARs, the potency of a chemical is strongly correlated to halogens on 3 or 4 lateral ring positions (Fig 1-2), at least one unsubstituted ring position, and the ability to assume a planar or coplanar conformation. This relationship is strongest when considered within one species and one class of chemicals. When one compound is compared across species receptor affinity is often similar while toxic potency varies widely. This may be due to alterations in the control of induction and/or members of the AhR gene battery (Poland and Knutson, 1982).

\section{Aryl Hydrocarbon Receptor (AhR) Signal Transduction Pathway}

\section{AhR Signal Transduction Pathway}

The Aryl hydrocarbon receptor (AhR) signal transduction pathway alters gene expression via a multi-step process (Fig 1-3). First a ligand must diffuse through the cytoplasmic membrane. Most ligands are non-polar and are not actively transported into the cell, but readily diffuse through the lipid rich membrane. Once in the cytoplasm the ligand can bind to AhR in its heat shock protein 90 (hsp90) bound form. This binding results in translocation of the AhR-ligand complex to the nucleus, the loss of hsp90s, and recruitment of the AhR nuclear translocator (ARNT). All of these events must occur before DNA binding can take place (Hankinson, 1995). The dimerization of AhR and ARNT, as well as binding of the dimer to DNA, maybe dependent on phosphorylation, possibly involving a tyrosine kinase (Gradin et al., 1994). 
The ligand-AhR-ARNT complex binds to DNA at specific sites, known as dioxin (or xenobiotic) responsive elements (DRE) (Swanson and Bradfield, 1993). There may be one or more DREs upstream of the target gene. After binding of one or more AhR-ARNT dimers to the target gene(s), transcription can be activated or suppressed. Activation of the AhR pathway is fairly rapid with peak recruitment of $A h R$, in mammalian hepatoma cells, occurring within 6 hrs of exposure. Following nuclear recruitment, AhR levels in the cell fall to approximately $20 \%$ of pre-exposure levels (Okey et al., 1994). In rats and mice, AhR function is downregulated following exposure to an AhR ligand. This suppression can be blocked by use of inhibitors of protein or mRNA synthesis, suggesting that this repression is mediated by an inducible factor (Hankinson, 1995). Elevated levels of target proteins will be maintained for varying lengths of time depending on a variety of factors, including message stability, protein stability, and rate of active degradation of the protein. 


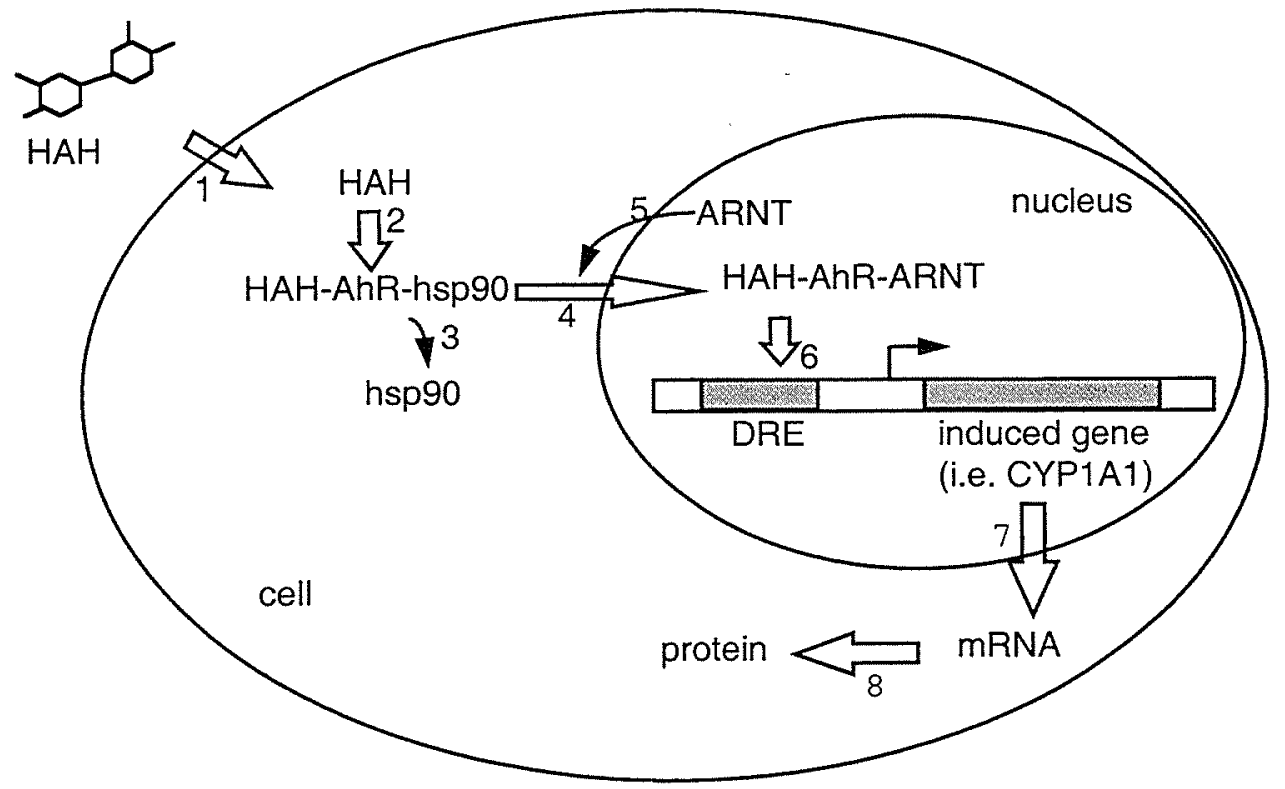

Fig. 1-3: AhR signal transduction pathway. Each of the sites in the pathway where a change could result in decreased sensitivity to AhR ligands are numbered. 1. Diffusion of the ligand into the cell. 2. Binding of ligand to AhR. 3. Migration into the nucleus. 4. Release of Hsp90 5. Binding to ARNT 6. Binding to the DRE. 7 \& 8. modified transcription and translation (Modified from (Hahn, 1998)) 


\section{PAS proteins}

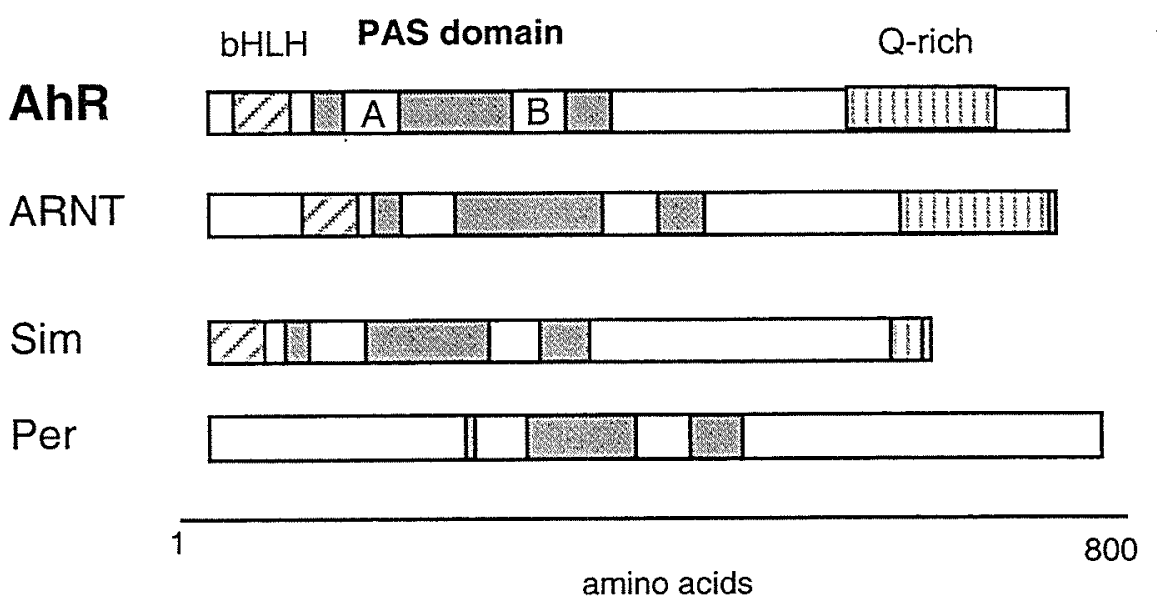

Fig. 1-4: AhR and ARNT protein structure. The features shown are those that are common to most members of the PAS family of proteins. bHLH is the basic helix-loophelix. The PAS domain contains two imperfect amino acid repeats, A and B. The $\mathrm{Q}$ rich region which has several glutamine rich regions. Per and Sim are Drosophila proteins. (Modified from Swanson and Bradfield, 1993) 


\section{Aryl Hydrocarbon Receptor (AhR) in Mammals}

The AhR is a cytoplasmic protein in the PAS family of proteins (reviewed in (Swanson and Bradfield, 1993)). The PAS family, named for the original members: Per (a Drosophila protein), ARNT (the AhR binding partner), and Sim (also in Drosophila), consists of proteins that have several common features (Fig 1-4). These features include a basic-helix-loop-helix (bHLH) region, which has a series of basic amino acids followed by two regions where the sequence implies helices will be formed. These two helices are joined by a "loop" of several amino acids. The PAS domain covers 200-300 amino acids and contains two regions of degenerate amino acid repeats (A and $\mathrm{B}$ ). The final section is a glutamine-rich (Q-rich) region near the carboxy terminus. Not all members of the family have the bHLH or the $\mathrm{Q}$ region.

The basic (b) region of the bHLH domain is involved with DNA binding, while the HLH part aids in dimerization with ARNT (Fukunaga and Hankinson, 1996). The AhR bregion is insufficient alone to bind with DNA, it must be paired with the b-region of ARNT for successful DNA binding to occur. The HLH pairing provides most of the strength to the AhRARNT bond, but the interaction between the two PAS regions determines the specificity of the pairing (Pongratz et al., 1998) and confers additional stability (Lindebro et al., 1995). The PAS region of the AhR is also involved in binding to ligand and HSP90. The Q-rich region is seen in many proteins which are involved in transcriptional activation (Okey et al., 1994). Functions are localized to these general regions but not exclusively, and one region may have multiple functions or a function may span parts of two (or more) regions.

The best understood cases of resistance mediated by differences in the AhR involve strains of inbred mice and mutant murine (Hepa-1) cell lines. The non-responsive mice have an allele of the AhR with an affinity for ligand that is generally 10 times lower than responsive mice (Okey et al., 1989). This lowered affinity has been attributed to 1 or 2 amino acid changes; one of which is located in the ligand binding region (Poland, 1994). Complementation assays with a number of non-responsive murine cell lines have shown that changes in other components of the AhR signal transduction pathway could result in lower induction without altering ligand affinity. One of these is linked to a change in ARNT (Hankinson, 1995).

AhR transcriptional activity is regulated in a tissue and gene dependent manner. Studies with human cell lines have indicated that non-responsive cell lines contain a constitutive DRE 
binding protein that prevents the AhR complex from binding to the DRE (Gradin et al., 1999). There is also a protein found in mice, called the AhR repressor (AhRR), which can inhibit the AhR pathway by competing with AhR for ARNT and/or DREs (Mimura et al., 1999). The $5^{\prime}$ flanking region of the AhRR gene contains multiple DREs suggesting that this protein can also be induced by HAHs. This implies the possibility that the alteration in Fundulus could be due to expression of a similar repressor protein in tissues where it is normally not expressed. A case of induction of a dioxin-dependent repressor of CYP1A1 transcription was reported by Watson et al (Watson et al., 1992).

\section{AhR in Fish}

AhRs have been found in a variety of bony, cartilaginous fish, and jawless fish (Hahn et al., 1997). Unlike in mammals, several fish species have been reported to have 2 AhRs, including Fundulus heteroclitus (Hahn et al., 1997). In killifish, these 2 AhRs are the products of distinct genes and are as divergent from each other as either is from mammalian receptors. These receptors have distinct patterns of expression. AhR 1 is expressed in a tissue specific pattern with the greatest amount of messenger RNA found in the brain, heart, and ovary and little or no expression in the liver, kidney, or gill. AhR2 is expressed ubiquitously (Karchner et al., 1999). These differences in expression patterns suggest that each of the AhR in killifish may have a distinct function.

Two studies have reported differences in CYP1Al inducibility which could be linked to differences in the AhR pathway. In Atlantic tomcod from the Hudson River CYP1A1 mRNA is not induced by treatment with HAHs but is induced by treatment with PAHs (Roy and Wirgin, 1997). Hepatic nuclear AhR binding to DREs was lower in Hudson River tomcod than in tomcod from a clean site when they were treated with $3,3^{\prime}, 4,4^{\prime}$-tetrachlorobiphenyl (TCB). There was no such difference when the fish were treated with benzo-a-pyrene. There was no difference in hepatic AhR mRNA expression between the Hudson River fish and fish from a reference site. A study in cell lines derived from rainbow trout reported an $\sim 3$-fold difference in AhR expression that corresponded to a difference in CYP1A1 inducibility (Pollenz and Necela, 1998). 


\section{AhR Influenced Genes}

Almost all of the work to date on genes influenced by the AhR has been done in mammalian systems. If the NBH killifish do have an alteration in the AhR pathway, comparisons between this population and responsive fish could be instrumental in investigating the differences between teleost and mammalian AhR influenced genes. In mammals, the AhR has been found to influence a wide spectrum of genes including some phase I and phase II xenobiotic metabolizing enzymes. In mice there are currently six genes which constitute the AhR "gene battery" (Nebert et al., 1993). These include genes encoding for two phase I enzymes (CYP1A1 and CYP1A2) and four phase II enzymes (NADPH: menadione oxidoreductase (Nmo-1), a tumor specific aldehyde dehydrogenase (ALHD-3), UDPglucuronyltransferase (UGT1*06), and a glutathione S-transferase (GST-Ya)). These genes are up-regulated by AhR and down-regulated in the presence of a functional CYP1A1/1A2 (Vasiliou et al., 1995).

AhR can also affect, directly or indirectly, a number of proteins involved with cell growth and differentiation. Included among these are transforming growth factor-alpha (TGFa) (Hankinson, 1995), epidermal growth factor (EGF) receptor (Lin et al., 1991), testosterone 7-hydroxylase, interleukin-1 (Sutter, 1992), fos, jun (Hoffer et al., 1996) and tumor necrosis factor (Clark et al., 1991). The effect of the AhR pathway on these proteins has not been examined in fish. AhR is believed to play a role monocyte function, and therefore in immune function. AhR mRNA is specifically induced during myelomonocytic differentiation ((Hayashi et al., 1995) and AhR knock-out mice have impaired immune systems (Fernandez Salguero et al., 1995).

\section{ARNT}

The Ah Receptor Nuclear Translocator (ARNT) binds to the AhR after ligand binding has occurred. The exact cellular location of ARNT is not known for certain, but evidence now suggests that ARNT is localized in the nucleus (Hankinson, 1995). It was originally thought that ARNT was involved in the translocation of the AhR-ligand complex to the nucleus, hence the name, but this is now known to be incorrect (Hoffman et al., 1991). ARNT is now known to be essential for AhR binding to DNA. It is also thought that ARNT plays an active role in 
the release of Hsp90 (Lindebro et al., 1995). ARNT is also capable of binding to other PAS family members, including hypoxia inducible factor (HIF-1 $\alpha$ ) (Wood et al., 1996).

In rodents, two distinct ARNT genes have been reported. ARNT1 is expressed ubiquitously (Hirose et al., 1996). Expression of ARNT2 is limited to the brains and kidney in adults.

ARNT, like AhR, is a member of the PAS family of proteins (Hoffman et al., 1991). Deletion experiments have indicated that the $\mathrm{BHLH}$ region is involved with dimerization with AhR (Whitelaw et al., 1993) and that PAS domain is involved with dimerization and/or DNA binding ( $\mathrm{Li}$ et al., 1994). In any case deletion of either region abolishes TCDD-induction. The presence of defective ARNT may be detected by the failure of ligand bound AhR to accumulate in the nucleus (Hoffman et al., 1991). What role ARNT may play in keeping AhR in the nucleus, beyond facilitating binding to DNA, is currently unknown.

\section{ARNT in Fish}

A few studies have reported on ARNT in fish. In killifish only one form of ARNT has been found, so far (Powell et al., 1999). This form is most similar to the rodent ARNT2s, but is expressed ubiquitously. Two splice variants of ARNT have been found in rainbow trout (Pollenz et al., 1996). One of these is a truncated form of ARNT which appears to have a dominant negative phenotype.

\section{DRE}

The DNA sequence that the AhR-ARNT dimer recognizes is known as the dioxin (or xenobiotic) responsive element (DRE). This sequence is found upstream of the promoter region of genes which are responsive to dioxin and dioxin like chemicals. The consensus sequence for this element is GGGNAT(C/T)GCGTGANNCC (Lusska et al., 1993). The motif required for binding is CGT* $G$. The presence of this motif only establishes binding, the sequence of surrounding nucleotides is important for activating transcription (Fukunaga and Hankinson, 1996). It is possible to mutate the DRE in mouse hepatoma cells to abolish transcriptional activation without changing binding to the DRE (Lusska et al., 1993). The AhR-ARNT binding sequence is different from the "e-box" sequence (CANNTG) that most bHLH proteins recognize (Hankinson, 1994). But x-ray crystallography indicates that ARNT 
contacts the DRE at the $\mathrm{T}^{*}$, thus the region that ARNT contacts is quite similar to the e-box half site (NTG). AhR is thought to contact 5 ' to the ARNT contact site in a site that does not resemble an e-box half-site (Fukunaga and Hankinson, 1996).

In general, multiple DRE's are found in a gene which is responsive to the AhR (Swanson and Bradfield, 1993). This multiplicity is thought to increase the chances that an activated receptor will be able to find a sequence in the chromatin to which it can bind (Wu and Whitlock, 1993). It has also been hypothesized that the multiple copies might play a role in the magnitude of the response the AhR pathway initiates (Denison et al., 1988).

\section{Cytochrome P450 1A1}

Cytochrome P450 1A1 (CYP1A1) is a membrane bound, heme protein, which catalyzes the addition of oxygen to planar or coplanar aromatic hydrocarbons. Elemental oxygen $\left(\mathrm{O}_{2}\right)$ is the oxygen source used. In the normal course of events electrons are passed from the initial donor (NADH or NADPH) to a secondary donor (NADH-cytochrome b5 reductase or NADPH-cytochrome P450 reductase, respectively). The secondary donor either passes both electrons to cytochrome b5 (NADH-cytochrome b5 reductase) or passes one electron to b5 and one to the P450 (reviewed in (Stegeman and Hahn, 1994)) (Fig. 1-5).

The addition of oxygen to the substrate can have different consequences depending on the nature of the substrate and the position to which the oxygen is added. The oxygenated intermediate may be more or less toxic than the original material and any one substrate may produce some metabolites that are more toxic and others that are less toxic. The oxygen handle on the substrate may be used by phase II xenobiotic metabolizing enzymes to add a large polar group resulting in an easily excreted metabolite. It has been hypothesized that the toxicity of some polyaromatic hydrocarbons $(\mathrm{PAH})$ is related to the extent to which the PAH, via the AhR, activates cytochrome P450s and inactivates phase II enzymes (Hankinson, 1995). Finally, some substrates (ex. dioxins) are very slowly metabolized perhaps due to difficulties in transferring oxygen from the $\mathrm{P} 450$ to the substrate. This difficulty may lead to the formation of active oxygen and indirectly cause toxicity.

For the purposes of this thesis the role of CYP1A1 as a biomarker is most important. A biomarker is generally accepted to be any biochemical, physiological or histological change 
used to estimate exposure to chemicals or the effects of such exposures (Huggett et al., 1992). Biomarkers may be important for their ability to bridge the gap between measured levels of contamination and the observed changes in environmental health. CYP1Al makes a good biomarker in this sense, because the mechanism of induction is relatively specific to a class of chemicals, and therefore should correlate well to the level of bioavailable toxicant. Also the mechanism of CYP1Al induction plays a role in the mechanism of toxicity of $\mathrm{HAH}$, creating a strong link to the observed state of environmental health.

The interpretation of any biomarker can be complicated, with intraspecies variability in response, and perhaps past exposure, influencing current responses. To interpret the biomarker we need to understand how these compounds exert their effects and how they interact with other environmental contaminants. A serious limitation to this interpretation is that we do not know what chronic exposure can do to expression of many biomarkers. 


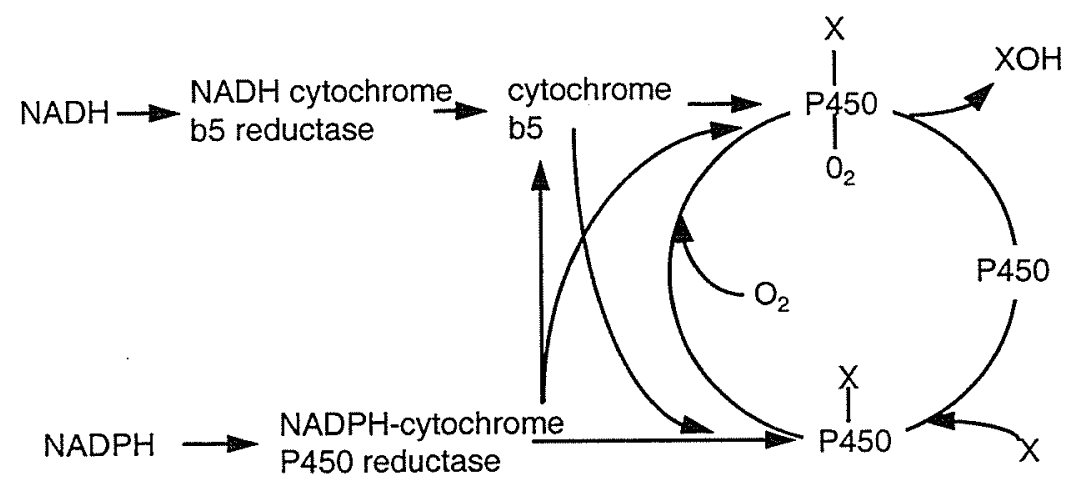

Fig. 1-5: CYP1A1 metabolism. X represents the xenobiotic compound. The $\mathrm{P} 450$ binds to $\mathrm{X}$ and then accepts an electron donated by either NADH or NADPH through their respective pathways. Oxygen then binds. A second electron is added. The $\mathrm{O}-\mathrm{O}$ bond is cleaved forming a substrate radical. This radical is hydroxylated and released (XOH) regenerating the unbound $\mathrm{P} 450$. 


\section{Glutathione S-Transferase}

Glutathione S-Transferases (GSTs) are a superfamily of phase II xenobiotic metabolizing enzymes that catalyze the reaction of the - $\mathrm{SH}$ group of glutathione with electrophilic sites of compounds such as PAHs and HAHs (reviewed in (Hayes and Pulford, 1995)). Mammalian GSTs are dimeric and 5 different families of subunits have been identified. Induction of the $\alpha$ subunit is mediated by the AhR. Induction of other subunits can be mediated by epoxides or steroid hormones. Dimers formed from the combination of different subunits have ligand specificities that may overlap. 1-Chloro-2, 4-Dinitrobenzene (CDNB) can be a ligand for most GSTs and is used to assess overall GST activity.

Induction studies in fish have yielded contradictory results. In Arctic char embryos and larvae, $\beta$-naphthoflavone (BNF) failed to change GST activity (Monod et al., 1996). In rainbow trout and cod, TCDD failed to change GST activity (Hektoen et al., 1994). GST expression in rainbow trout was decreased by $2,3,3^{\prime}, 4,4^{\prime}$-pentachlorobiphenyl (Bernhoft et al., 1994), but was increased by 3, 3', 4, 4'-tetrachlorobiphenyl (Otto and Moon, 1995). GST activity was induced in carp by exposure to 3-methylcholanthrene (3MC) (Taysse et al., 1998). These all used CDNB as the substrate and therefore integrated the activity of multiple GSTs. A plaice GST-A gene has been sequenced and found to have 2 DREs suggesting that AhR may mediate changes in expression of this gene (Leaver and George, 1995).

\section{UDP-glucuronysyltranserase}

UDP-glucuronysyltranserases (UGTs) are a superfamily of phase II xenobiotic metabolizing enzymes that catalyze the reaction of the glucuronic acid with nucleophilic compounds such as phenols, quinols, and $\mathrm{N}$-oxidized aromatic amines (reviewed in (Bock, 1991)). Many substrates can be acted on by more than one type of UGT. Induction of the UGT enzymes with activity towards phenol is mediated by the AhR pathway. UGT activity can also be changed by steroid hormones and antioxidants.

Studies in fish have again yielded mixed results. UGT activity was induced in sea bass by BaP (Lemaire et al., 1992). BNF exposure did not affect UGT activity in rainbow trout (Koponen et al., 1997). UGT activity in rainbow trout was induced by 3, 3', 4, 4'tetrachlorobiphenyl and 2, 3, 3', 4, 4'-pentachlorobiphenyl (Huuskonen et al., 1996). All of 
these studies used 4-nitrophenol, which can be metabolized by most UGTs. One study in plaice using multiple substrates demonstrated that UGT activity for 1-naphthol but not bilirubin or testosterone was induced by exposure to 3MC or Aroclor 1254 (Clarke et al., 1992).

\section{$\underline{\text { Specific Aims }}$}

To assess the impacts of multigenerational exposures to high concentrations of HAHs, a population of killifish ( $\mathrm{F}$. heteroclitus) from New Bedford Harbor $(\mathrm{NBH})$, a Superfund site, were examined. $\mathrm{NBH}$ is contaminated with PCBs, polychlorinated-dibenzofurans (PCDF), polychlorinated-dibenzo-p-dioxins (PCDD), and many heavy metals. $\underline{\mathrm{F}}$. heteroclitus from NBH have extremely high whole body PCB levels, averaging $1370 \mathrm{ug} / \mathrm{g}$ for fish caught close to the source of contamination (Lake, 1995). In spite of this body burden, these fish seem to be able to live and reproduce without major problems. Fundulus were selected because of the broad background of knowledge about their behavior, biochemistry, and gene expression. The relative isolation of Fundulus populations makes them good candidates to develop resistance following prolonged exposures to toxic chemicals. Given the exposure of NBH fish and the negative effects of PCBs we hypothesized that the NBH killifish had developed resistance to HAHs. The specific questions I addressed to investigate this hypothesis were:

1a. Are the expression of phase I and phase II xenobiotic metabolizing enzymes in Fundulus from NBH different from the expression of these enzymes in fish from a reference site (Scorton Creek, MA; SC)?

1b. Does expression of these enzymes change after the NBH fish are removed from NBH?

2a. Does induction of these enzymes by HAH differ between NBH and SC fish?

2b. Does induction of these enzymes by PAH differ between NBH and SC fish?

3. Are differences in inducibility between NBH and SC fish heritable?

4. Is there a difference in AhR binding between NBH and SC fish? 
Each of the questions is addressed in the following chapters. In Chapter 2, the expression of CYP1Al and the activity of GST and UGT is characterized in killifish from SC and NBH shortly after collection (1a). The expression of CYP1A1 and activity of GST and UGT were also monitored over the first 90 days after collection (1b). As part of the characterization of the expression of these enzymes in field fish, the PCB concentrations and TCDD-equivalents were determined in SC and NBH fish; this allowed for comparisons in enzyme expression to be related to relative concentrations of inducers. In Chapter 3 , the inducibility of CYP1A1, GST, and UGT by 2,3,7,8-TCDF was examined in feral female and male SC and NBH fish (2a). The inducibility of CYP1A1 by 2,3,7,8-TCDD and BNF was also examined in primary cultures of hepatocytes from feral SC and NBH fish ( $2 \mathrm{a} \& 2 \mathrm{~b}$ ). In Chapter 4, the inducibility of CYP1A1, GST, and UGT by $2,3,7,8-\mathrm{TCDF}$ was examined in $\mathrm{NBHF}_{1}$ fish (3). The inducibility of CYP1A1 by ${ }^{3} \mathrm{H}-2,3,7,8-\mathrm{TCDD}$ and BNF was examined in SC and NBH $F_{1}$ fish (3). In Chapter 5, AhR binding in hepatic cytosols from feral SC and NBH fish and in TCDF-treated and control $\mathrm{NBH}_{1}$ fish are compared (4). Chapter 6 summarizes the findings reported in Chapters 2 through 5 and provides some speculation on future directions and the possible repercussions of acquired resistance. 
Chapter 2: EXPRESSION OF XENOBIOTIC METABOLIZING ENZYMES AND CHARACTERIZATION OF PCB CONCENTRATIONS IN AN ESTUARINE TELEOST (FUNDULUS HETEROCLITUS) FROM THE NEW BEDFORD HARBOR, MA SUPERFUND SITE.

Introduction $\quad 46$

Materials and Methods $\quad 48$

$\begin{array}{ll}\text { Materials } & 48\end{array}$

Fundulus Collection and Maintenance $\quad 48$

$\begin{array}{ll}\text { Tissue Collections } & 48\end{array}$

Microsomal Preparation 49

Enzyme Activity Assays $\quad 50$

Protein and RNA Blotting $\quad 51$

IHC 52

PCB Analysis $\quad 52$

Results $\quad 55$

Characteristics of SC and NBH Fundulus $\quad 55$

PCB Analysis $\quad 55$

$\begin{array}{ll}\text { CYP1A1 Activity and Expression } & 70\end{array}$

GST Activity $\quad 80$

UGT Activity $\quad 80$

$\begin{array}{ll}\text { Discussion } & 86\end{array}$ 


\section{Introduction}

New Bedford Harbor (NBH, Fig. 1-1), MA, is a federal Superfund site contaminated with polychlorinated biphenyls (PCBs), dibenzofurans ( $\mathrm{PCDFs}$ ), dioxins (PCDDs) and heavy metals. Concentrations of PCBs in NBH sediment range from a few $\mathrm{ppm}$, in the lower harbor, up to $100,000 \mathrm{ppm}$ in the area closest to the source of contamination (the hot spot) (Weaver, 1984) Lake (Lake, 1995) examined PCB concentrations in intact carcasses of several species from NBH and reported that Fundulus heteroclitus from the hotspot had the highest body burdens, with total PCB body burdens averaging $1370 \mathrm{ppm}$ (dry weight). Despite having such high PCB concentrations, these fish continue to survive and reproduce (Gleason et al., 1997).

Planar halogenated aromatic hydrocarbons (pHAHs), such as those found in $\mathrm{NBH}$, elicit a number of toxic responses including immunotoxicity, porphyria, thymic atrophy, and hepatocarcinomas. Most of these responses are mediated through the aryl hydrocarbon receptor (AhR). The planar or coplanar HAHs that elicit these AhR-mediated responses are also referred to as dioxin-like-compounds (DLCs). The sensitivity of an organism to 2,3,7,8-tetrachlorodibenzo-p-dioxin (TCDD) is commonly used to compare the responsiveness of different organisms to HAHs. Activation of the AhR signal transduction pathway has been linked to the toxicity of DLCs in mammals (Poland and Knutson, 1982). For example, studies in mice have shown that strains with AhRs having lower affinity for DLCs (Okey et al., 1989) (Hori et al., 1997) and AhR null strains (Fernandez Salguero et al., 1996) are less sensitive to the toxic effects of these compounds. The results in the AhR null mice suggest that all of the pathological changes normally seen in the livers of TCDD-treated mice are mediated by the AhR.

Fundulus have been shown to possess all the components of a functional AhR signal transduction pathway (Hahn et al., 1997; Karchner et al., 1999; Powell et al., 1999) that functions in the same manner as the mammalian AhR pathway. The typical mammalian biochemical response to exposure to TCDD is the induction of a suite of proteins, including cytochrome P4501A1 (CYP1A1) and certain members of the 
glutathione-S-transferase (GST) and UDP-glucuronosyltransferase (UGT) families (Sutter, 1992). The primary evidence for the functionality of the Fundulus AhR signal transduction pathway is the inducibility of CYP1A1 following either laboratory (KloepperSams and Stegeman, 1989; Willett et al., 1995) or field exposures (Burns, 1976; Elskus, 1989) to many of the same compounds that are known to be inducers in mammals.

We hypothesize that NBH Fundulus exposed to high PCB concentrations for multiple generations may have developed biochemical adaptations to minimize the toxicological effects of these compounds. Support for this hypothesis comes from the prior reports of Fundulus developing resistance following exposure to a host of chemicals, including mercury (Weis and Weis, 1989), creosote/PAHs (Van Veld and Westbrook, 1995) and dioxins (Prince, 1995b). In this paper we examine some of the biochemical endpoints of HAH exposure in NBH Fundulus for evidence of changes in expression patterns that could indicate decreased sensitivity to $\mathrm{HAH}$.

We chose to examine the expression of genes thought to be regulated by the AhR pathway in the NBH fish. This approach is based on the presence of a functional AhR pathway in Fundulus and its importance in the toxicity of HAH. Induction of CYP1A1 is the clearest marker of activation of the AhR induction pathway in teleosts, but the responses of other xenobiotic metabolizing enzymes potentially regulated by the $A h R$ pathway were also of interest. We examined the expression of CYP1A1 (a phase I xenobiotic metabolizing enzyme) and two phase II xenobiotic metabolizing enzyme families, GST and UGT, in Fundulus from NBH and a reference site, Scorton Creek (SC). To determine if any observed site specific differences were systemic or tissue specific, responses were examined in both hepatic and extrahepatic tissues. The PCB content of carcasses and livers of animals from both sites were measured to determine the relative contamination of fish from each site. 


\section{Materials and Methods}

Materials: Frozen krill was purchased from MidJersey Pet Supply (Carteret, NJ). APconjugated $2^{\circ} \mathrm{Ab}$ goat anti-mouse was purchased from Bio-Rad (Hercules, CA). Chemiluminescent substrates were purchased from S\&S (Keene, NH) and Tropix (Bedford, MA). UPC-10 Ab was purchased from Organon Teknika (West Chester, PA). A universal immunoperoxidase kit (murine) was purchased from Signet Laboratories (Dedham, MA) ${ }^{32} \mathrm{P}$ was purchased from Dupont NEN (Boston, MA). RNA STAT-60 ${ }^{\text {TM }}$ was purchased from TEL-Test (Friendswood, TX). Permount, SDS and acrylamide were purchased from Fisher (Pittsburgh, PA). All other chemicals were purchased from Sigma (St Louis, MO).

F. heteroclitus collection and maintenance: NBH fish were collected by using minnow traps baited with dog food (6/28 - 6/29/94) or by Ken Rocha at the EPA's Narragansett office (9/8/97) using unbaited minnow traps and given to us immediately after collection. SC fish were collected with minnow traps baited with dog food (7/18/94 \& 7/15/97). Fish were maintained in $20^{\circ} \mathrm{C}$ flowing sea water and fed Tetramin ${ }^{\circledR}$ stapleflake and minced krill (NJ pet supply).

1994 and 1997 sampling: Tissues were sampled as described in Table 1. Tissues for microsomal preparation (see below) were placed immediately into ice cold AhR buffer. Tissues for RNA were frozen in liquid nitrogen and then stored at $-80^{\circ} \mathrm{C}$ until prepared. Tissues for IHC were placed in 10\% neutral buffered formalin (NBF) until embedded. 
Table 1: Number of fish used per site for each tissue for each method of tissue preparation in 1994 and 1997.

\begin{tabular}{|l|l|l|l|l|l|l|l|l|l|}
\hline $\begin{array}{l}\text { Tissue } \\
\text { Method }\end{array}$ & Year & Liver & Heart & Spleen & Kidney & Intestine & Gill & Gonad & Carcass \\
\hline $\begin{array}{l}\text { Micros- } \\
\text { ome \& } \\
\text { Cytosol }\end{array}$ & 1994 & 8 & & & & & & & \\
\hline & 1997 & 12 & $24^{*}$ & & $24^{*}$ & $24^{*}$ & $24^{*}$ & & \\
\hline \multirow{2}{*}{ IHC } & 1994 & 4 & 8 & & & 8 & 8 & & \\
\cline { 2 - 10 } & 1997 & 6 & 6 & 6 & 6 & 6 & 6 & 6 & \\
\hline RNA+ & 1994 & & & & & & & & \\
\cline { 2 - 10 } & 1997 & 12 & 12 & 12 & 12 & 12 & 12 & 12 & \\
\hline \multirow{2}{*}{$\begin{array}{l}\text { Chemis } \\
\text {-try. }\end{array}$} & 1994 & & & & & & & & 12 \\
\cline { 2 - 10 } & 1997 & $24^{* *}$ & & & & & & & \\
\hline
\end{tabular}

The total number of fish analyzed in 1994 or 1997 per site is presented in each of the columns (liver through carcass). *Heart, kidney, intestine and spleen of SC fish were pooled into 6 pools per tissue, 3 male and 3 female, with 4 fish in each pool. There were insufficient NBH male fish caught so there were only 4 pools per tissue, 3 female and 1 male, with 4 fish per pool. + Tissues for RNA preparation were pooled from two fish of the same sex from the same site. ${ }^{* *}$ In 1997, the total number of livers for NBH was 16 (12 female, 4 male). Livers from SC fish were pooled into 3 pools ( 8 fish per pool). Livers from NBH fish were pooled into 4 pools ( 3 fish per pool).

Microsome and Cytosol preparation: Tissues were homogenized in $9 \mathrm{ml}$ of cold AhR buffer per gram of tissue. AhR buffer consists of $25 \mathrm{mM}$ MOPS ( $\mathrm{pH}$ 7.5) with $1 \mathrm{mM}$ EDTA, 5 mM EGTA, $0.02 \% \mathrm{NaN}_{3}, 20 \mathrm{mM} \mathrm{Na}_{2} \mathrm{MoO}_{4}, 10 \%$ (v:v) glycerol, $1 \mathrm{mM}$ dithiothreitol, plus protease inhibitors ( $20 \mathrm{uM}$ TLCK, $5 \mu \mathrm{g} / \mathrm{ml}$ leupeptin, $13 \mu \mathrm{g} / \mathrm{ml}$ aprotinin, $7 \mu \mathrm{g} / \mathrm{ml}$ pepstatin A, and $0.1 \mathrm{mM} \mathrm{PMFS}$ ) (Hahn et al., 1994). Tissues were 
homogenized with a Teflon-glass homogenizer (10 passes); intestines and gills were minced with dissecting scissors prior to homogenization. Homogenates were centrifuged for $10 \mathrm{~min}$ at $750 \mathrm{~g}$ and $10 \mathrm{~min}$ at $12,000 \mathrm{~g}$ at $4^{\circ} \mathrm{C}$. The supernatant was then centrifuged at $100,000 \mathrm{~g}$ for $70 \mathrm{~min}$ at $4^{\circ} \mathrm{C}$. This supernatant (cytosol) was removed and frozen in liquid $\mathrm{N}_{2}$. The pellet (microsomes) was resuspended in TEDG $(0.05 \mathrm{M}$ Tris pH 7.5, $1 \mathrm{mM}$ EDTA, $1 \mathrm{mM}$ dithiothreitol, and 20\% glycerol) (Stegeman et al., 1979) and frozen in liquid $\mathrm{N}_{2}$.

EROD activity: CYP1A1 activity was determined using a fluorometric, kinetic assay for EROD activity (Hahn et al., 1993). Assays were run in 48 well plates with $2 u \mathrm{M} 7$-ethoxy resorufin and 1.0 mM NADPH (final concentrations). Samples were scanned once every minute for 15 minutes. A standard resorufin curve (ranging from 0 to $200 \mathrm{pmol}$ of resorufin, with 4 replicates of each concentration) was run with each set of samples. EROD activity is presented as pmol resorufin per minute per mg total microsomal protein. Samples were run in duplicate. EROD activity in individual wells was linear for at least 10 minutes.

Total protein content: Total microsomal or cytosolic protein was measured fluorometrically (Lorenzen and Kennedy, 1993).

GSH transferase (GST) activity: The protocol described in Habig etal (Habig et al., 1974) as modified by Van Veld etal (Van Veld et al., 1991)was followed, with modifications. Briefly, GST activity was measured by adding cytosol ( 0.02 to $0.14 \mathrm{mg}$ total protein) to $1 \mathrm{ml}$ of reaction buffer containing $1 \mathrm{mM} \mathrm{CDNB}, 100 \mathrm{mM}$ Tris- $\mathrm{HCl}$, and $1 \mathrm{mM}$ reduced glutathione and then monitoring the change in absorbance for 1 minute at $344 \mathrm{nM}$. GST activity was expressed as nmol of conjugate produced per minute per mg total cytosolic protein. UDP Glucuronosyl Transferase (UGT) activity: The protocol described by Andersson etal (Andersson et al., 1985) was followed, with modifications. Briefly, microsomes (0.01 to 0.11 mg total protein) were added to $0.5 \mathrm{M} \mathrm{KH}_{2} \mathrm{PO}_{4}$ with $p$-nitrophenol $(2.43 \mathrm{mg} / 50 \mathrm{ml})$ and digitonin $(2 \mathrm{mg} / \mathrm{ml})$, plus or minus UDPGA ( $4 \mathrm{mg} / \mathrm{ml}$ ). This mixture was incubated for $20 \mathrm{~min}$ at RT, shaking in the dark. Then $0.45 \mathrm{ml}$ of $3 \%$ trichloroacetic acid was added and the entire mix centrifuged for $15 \mathrm{~min}$ at $4000 \mathrm{rpm}$. $\mathrm{KOH}(0.05 \mathrm{ml}$ of $5 \mathrm{M})$ was added to $0.375 \mathrm{ml}$ of the 
supernatant and the absorbance was read at $400 \mathrm{~nm}$. UGT activity was expressed as nmol of conjugate produced per minute per mg total microsomal protein.

Immunoblotting: CYP1A1 protein content was measured with a chemiluminescent western blot assay (Hahn et al., 1996) using the Scup 1-12-3 monoclonal antibody (MAb) (Park et al., 1986). A standard curve (0.025 to 0.5 pmol CYP1A1) using scup microsomes with known CYP1A1 content was used to determine CYP1A1 protein in the NBH and SC samples.

Samples were loaded so that they fell within the range of the standard curve. Samples were run either on $6 \%$ to $15 \%$ acrylamide gradient gels or $10 \%$ acrylamide non-gradient gels. Proteins were transferred to nylon membranes and then incubated with non-specific blocking solution (S\&Sß blocking powder). Membranes were then incubated with MAb 1-12-3 (10 ug/ml) for 1 hr, washed 3 times ( 3 water rinses then a 5 min wash with $100 \mathrm{ml}$ of tris buffered saline (TBS)) and incubated for $1 \mathrm{hr}$ with $2^{\circ} \mathrm{Ab}$ (AP-conjugated goat anti-mouse, Bio-Rad, 1.1:1000 dilution). Finally the membrane was washed 3 times again, exposed to a chemiluminescent substrate and placed on x-ray film (Kodak, AR). Multiple exposures were taken of each blot. RNA preparation \& dot blotting: Total RNA was prepared from frozen tissues using the RNA STAT-60 ${ }^{\text {TM }}$ (TEL-TEST; Friendswood, TX) protocol with modifications. Briefly tissue was homogenized in RNA STAT- $60^{\mathrm{TM}}$ ( $1 \mathrm{ml}$ per 50 to $100 \mathrm{mg}$ of tissue) then incubated at RT for $\sim 5 \mathrm{~min}$. Chloroform was added $\left(0.2 \mathrm{ml}\right.$ per ml RNA STAT $\left.-60^{\mathrm{TM}}\right)$, mixed by inverting and incubated for 2 to $3 \mathrm{~min}$ at $\mathrm{rt}$. This mix was then centrifuged for $15 \mathrm{~min}$ at $4^{\circ} \mathrm{C}$ at $12,000 \mathrm{~g}$ and the aqueous portion transferred to a new centrifuge tube. Isopropanol $(0.5 \mathrm{ml}$ per $1 \mathrm{ml}$ RNA STAT $-60^{\mathrm{TM}}$ ) was added, mixed by inverting, and incubated for 5 to $10 \mathrm{~min}$ at RT. This mixture was centrifuged for $30 \mathrm{~min}$ at $4^{\circ} \mathrm{C}$ at $12,000 \mathrm{~g}$. The supernatant was poured off and the pellet was washed with ice cold $75 \%$ ethanol and air dried. The pellet was dissolved in DEPC-treated water and stored at $-80^{\circ} \mathrm{C}$. RNA (10 or $20 \mathrm{ug}$ ) diluted in $6 \mathrm{X} \mathrm{SSC}(20 \mathrm{X} \mathrm{SSC}=3 \mathrm{M} \mathrm{NaCl}, 0.3$ $\mathrm{MNa}$ citrate, $\mathrm{pH} 7.0$ ) and 7.4\% formaldehyde was applied to nitrocellulose using the Milliblot dotblot system (Hahn and Stegeman, 1994). The dotblot was dried, exposed to UV light to crosslink the RNA, and probed with ${ }^{32} \mathrm{P}$ labeled $\underline{\mathrm{F}}$. heteroclitus full length CYP1A1 (Morrison 
et al., 1998). Positive (TCDF treated $\underline{\mathrm{F}}$. heteroclitus gill RNA) and negative controls (untreated $\underline{F}$. heteroclitus gill RNA) were run with each blot.

Immunohistochemistry (IHC): IHC was done according to the methods of Smolowitz et al (Smolowitz et al., 1991), with modifications. In brief, sections were deparaffinated and hydrated ending in $1 \%$ bovine serum albumin in phosphate buffered saline (PBS/BSA). The sections were inserted into Shandon cover slips and incubated with normal goat serum for 5 minutes to block nonspecific binding of the secondary antibody. Two 1-hour incubations with $150 u \mathrm{~L}$ of Monoclonal antibody $1-12-3(1.7 \mathrm{ug} / \mathrm{ml}$ in PBS/BSA) were done. The specificity of this antibody for CYP1A has been shown previously (Miller et al., 1989). Sections were washed with PBS/BSA after this step and the two following steps. The sections were incubated with secondary antibody (Goat antimouse IgG, 1/200 dilution) for 20 minutes, followed by peroxidase-linked mouse IgG, also for 20 minutes. After washing with PBS/BSA and then PBS, two 15 minute incubations (150uL each) of color developer (Signet) were performed. Sections were then washed with water, removed from the Shandon covers, and washed twice with water. Sections were next counter stained with Mayers hematoxylin and mounted in crystalmount. After drying, coverslips were attached with permount. Sections of induced and uninduced scup liver were run with each batch of sections as a positive and negative control, respectively. Matching sections were stained with a nonspecific IgG (purified mouse myeloma protein, UPC-10, Organon Teknika, West Chester, PA, $1.7 \mathrm{ug} / \mathrm{ml}$ in PBS/BSA) as a negative control. Sections were read blind and scored on two scales, occurrence: 0 (no cells staining) to 3 (all cells staining) and intensity: 0 (no staining) to 5 (very dark red staining). These 2 scores were multiplied for a final score ("staining index") of 0 to 15.

PCB analysis: Decapitated, eviscerated carcasses or liver pools were homogenized using a virtishear (Virtis). To improve fluidity of the sample during homogenization, 1-2 $\mathrm{ml}$ of methylene chloride-extracted DI water was added to the tissue. An aliquot of the homogenate was taken for wet weight/dry weight determination prior to being dried with sodium sulfate $(4: 1$, sodium sulfate:tissue). The dried tissue/sodium sulfate mixture was 
then pulverized with a mortar and pestle, and soxhlet extracted overnight in a 1:1 mixture of acetone:hexane. (An aliquot of this extract was saved to determine TEQs.) This extracted was then exchanged into hexane, spiked with PCB congener IUPAC 143 (Ballschmiter and Zell, 1980) as surrogate standard and layered onto a chromatography column packed with $5 \%$ deactivated alumina $/ 5 \%$ deactivated silica. The sample was eluted with $70 \mathrm{ml}$ of hexane to obtain the PCB-containing fraction, volume reduced, exchanged to $1 \mathrm{ml}$ in heptane and injected onto a gas chromatograph (HP5890 SERIES II) fitted with a $30 \mathrm{~m} \mathrm{db}-5$ column and an electron capture detector. Octachloronaphthalene was added just prior to injection to determine recoveries. Total PCB content was measured as a sum of 45 congeners, quantified using a standard mixture of AROCLORS 1232, 1248, and $1262(25: 18: 18)$. Values presented are corrected for recovery. Average recovery was $57 \%$ ( $\min .=30 \%, \max .=\sim 80 \%)$. PCB concentrations were expressed on a dry weight basis. Dioxin Equivalents: The soxhlet extracts were transferred to DMSO from the acetone : hexane mixture prior to dilution. Serial dilutions $(0.001-1.0$, where 1.0 is undiluted extract) in DMSO were prepared from each of the liver extracts ( $\mathrm{SC} \mathrm{N}=3, \mathrm{NBH} \mathrm{N}=4$ ). Expanded dilution series $(0.0001-1.0)$ were run for one extract from each site in a preliminary range finding experiment. Each extract was run on duplicate plates. Three replicate wells were exposed at each concentration on each plate. PLHC cells were plated in 96-well plates at $>1.3 \times 10^{6}$ cells $/ \mathrm{ml}$ and allowed to attach overnight. The final concentration of extract plus carrier (DMSO) was $0.5 \%$ of the total volume. The PLHC cells were then exposed to dilutions of the soxhlet extracts or TCDD $(0.001-30 \mathrm{nM})$ in DMSO or DMSO alone for 24 hours in serum free media, using methods described previously (Hahn et al., 1996).

EROD activity and total protein were determined using a fluorometric, 2-point kinetic assay for EROD activity and total protein ((Kennedy et al., 1995) with modifications by (Hahn et al., 1996)). Briefly medium with inducers was removed and the cells were washed with phosphate-buffered saline ( $136 \mathrm{mM} \mathrm{NaCl}, 0.81 \mathrm{mM} \mathrm{Na} 2 \mathrm{HPO} 4,0.15 \mathrm{mM} \mathrm{KH} 2 \mathrm{PO} 4,0.27$ mM KCL). Phosphate buffer ( $25 \mathrm{mM} \mathrm{Na2HPO4-7H2O} \mathrm{/} \mathrm{NaH2PO4-H2O)} \mathrm{and} \mathrm{7-ER} \mathrm{(} 2 \mathrm{uM}$ ) 
were added to each sample well and an initial reading (time 0 ) was taken. This mix was then incubated for $8 \mathrm{~min}$ at $\mathrm{rt}$ and a final reading taken. The reaction was stopped with the addition of cold fluorescamine in acetonitrile $(4.04 \mathrm{nM})$. The plates were then incubated for $15 \mathrm{~min}$ at $\mathrm{rt}$ and scanned. BSA and resorufin standards were run on each plate. Dioxin equivalents were determined by dividing the EC50 for TCDD (determined in the same experiment) by the EC50 for each extract. 


\section{Results}

\section{Characteristics of NBH and SC fish:}

LSI, GSI total body weight and microsomal yield are reported in Table 2. In 1994 and 1997, there was no significant difference in LSI or in total body weight between sites or sexes. In 1994 there was no significant difference in GSI between site or sex, but in 1997 there was a significant difference in GSI between sites, although not between sexes. Microsomal yield was significantly higher in livers from SC fish than in livers from NBH fish in 1997 ( $\mathrm{p}<0.001)$, but not in 1994. The microsomal yield was also significantly higher in the extrahepatic tissues of SC fish compared to extrahepatic tissues from NBH fish.

\section{PCB Analysis:}

Total PCB levels (the sum of the 45 congeners analyzed) in the carcasses of NBH fish were $\sim 1500$-fold higher than PCB levels in carcasses of SC fish (272 ug/g vs 0.177 ug/g dry weight) (Table $2, \mathrm{p}<0.005$ ). There was no significant difference in total PCB accumulation between the sexes at either site, although the concentration of some congeners did differ significantly between the sexes in NBH fish (Table 3 ). The contribution of individual PCB congeners to the total PCB body burden differed significantly between the two sites (Fig. 2-1A). The lower chlorinated congeners contributed more to the total PCB concentration in the carcasses of NBH fish, while the higher chlorinated congeners contributed more to the total PCB concentration in the carcasses of SC fish.

Similar results to those found in the carcasses were obtained for the concentrations of PCBs in the livers of NBH and SC fish. Total PCB concentrations in the livers of NBH fish $(809 \mathrm{ug} / \mathrm{g}$ ) were 726 -fold higher than PCB concentrations in the livers of SC fish $(1.11 \mathrm{ug} / \mathrm{g})($ Table 4$)$. Again, there were significant differences in the contribution of individual congeners to the total liver PCB concentration (Fig. 2-1B); with the lower 
chlorinated congeners contributing proportionally more to the total PCB concentration in the livers of NBH fish and the higher chlorinated congeners contributing proportionally more to the total PCB concentration in the livers of SC fish. Within site comparisons of the carcass congener distributions (on the basis of the number of chlorines) to the liver distributions reveals a distinct tissue related difference in the SC fish (Fig 2-2A) but not in the NBH fish (Fig 2-2B). The presence of this tissue related difference may obscure any changes in congener concentrations that might have resulted from changing field conditions.

Dioxin equivalents in soxhlet extracts of livers from $\mathrm{NBH}$ fish were significantly higher than in livers from SC fish (Table 2). None of the extracts from the livers of SC fish induced EROD activity in the PLHC cells at any of the concentrations tested (Fig. 2-3). All of the extracts from the livers of NBH fish induced EROD activity in the PLHC cells (EC50 $=0.004-0.03 \mathrm{mg}$ liver, wet weight). Dioxin equivalents could only be determined for the livers from NBH fish; these ranged from $\sim 8,000-70,000 \mathrm{pg}$ TCDD / g liver, wet weight. These TEQs are orders of magnitude higher than the FDA guideline for the edible portions of fish ( $25 \mathrm{pg} \mathrm{TCDD} / \mathrm{g}$ ) 
Table 2: Characterization of NBH and SC Fundulus collected in 1994 and 1997.

\begin{tabular}{|l|c|c|c|c|}
\hline \multicolumn{1}{|c|}{ Year of Collection } & \multicolumn{2}{|c|}{1994} & \multicolumn{2}{c|}{1997} \\
\hline \multicolumn{1}{|c|}{ Collection site } & SC & NBH & SC & NBH \\
\hline Body weight (g) & $\begin{array}{c}9.29 \pm 1.10 \\
(12)\end{array}$ & $\begin{array}{c}7.00 \pm 0.95 \\
(12)\end{array}$ & $\begin{array}{c}6.80 \pm 0.32 \\
(42)\end{array}$ & $\begin{array}{c}6.87 \pm 0.50 \\
(34)\end{array}$ \\
\hline LSI (\%) & $\begin{array}{c}2.91 \pm 0.48 \\
(12)\end{array}$ & $\begin{array}{c}3.51 \pm 0.66 \\
(12)\end{array}$ & $\begin{array}{c}2.31 \pm 0.48 \\
(42)\end{array}$ & $\begin{array}{c}2.33 \pm 0.09 \\
(34)\end{array}$ \\
\hline GSI (\%) & $\begin{array}{c}8.81 \pm 1.70 \\
(12)\end{array}$ & $\begin{array}{c}5.81 \pm 0.74 \\
(12)\end{array}$ & $\begin{array}{c}4.25 \pm 0.42 \\
(34)\end{array}$ & $\begin{array}{c}0.61 \pm 0.06^{*} \\
(34)\end{array}$ \\
\hline $\begin{array}{l}\text { Microsomal Yield (mg } \\
\text { protein / g liver) }\end{array}$ & $\begin{array}{c}24.18+3.27 \\
(8)\end{array}$ & $\begin{array}{c}17.23+2.58 \\
(8)\end{array}$ & $\begin{array}{c}12.18+1.48 \\
(12)\end{array}$ & $\begin{array}{c}7.62 \pm 0.48^{*} \\
(12)\end{array}$ \\
\hline $\begin{array}{l}\text { Microsomal Yield (mg } \\
\text { protein / g heart) }\end{array}$ & nd & nd & $\begin{array}{c}7.15 \pm 0.76 \\
(6)\end{array}$ & $\begin{array}{c}3.88 \pm 0.63^{*} \\
(4)\end{array}$ \\
\hline $\begin{array}{l}\text { Microsomal Yield (mg } \\
\text { protein / g kidney) }\end{array}$ & nd & nd & $\begin{array}{c}15.41 \pm 1.46 \\
(6)\end{array}$ & $\begin{array}{c}4.35 \pm 0.47^{*} \\
(4)\end{array}$ \\
\hline $\begin{array}{l}\text { Microsomal Yield (mg } \\
\text { protein / g gill) }\end{array}$ & nd & nd & $\begin{array}{c}18.47 \pm 4.85 \\
(6)\end{array}$ & $\begin{array}{c}3.94 \pm 0.41^{*} \\
(4)\end{array}$ \\
\hline $\begin{array}{l}\text { Microsomal Yield (mg } \\
\text { protein / g intestine) }\end{array}$ & nd & nd & $\begin{array}{c}13.41 \pm 2.41 \\
(6)\end{array}$ & $\begin{array}{c}4.56 \pm 0.42^{*} \\
(4)\end{array}$ \\
\hline $\begin{array}{l}\text { Liver Total PCB (ug /g } \\
\text { dry weight) }\end{array}$ & nd & nd & $\begin{array}{c}1.11 \pm 0.13 \\
(3)\end{array}$ & $\begin{array}{c}809 \pm 215^{*} \\
(4)\end{array}$ \\
\hline $\begin{array}{l}\text { Liver TEQ's (pg TCDD } \\
\text { /g liver wet weight) }\end{array}$ & nd & nd & $\begin{array}{c}\text { ud } \\
(3)\end{array}$ & $\begin{array}{c}25,000 \pm \\
15,000(4)\end{array}$ \\
\hline $\begin{array}{l}\text { Carcass Total PCB (ug / } \\
\text { g dry weight) }\end{array}$ & $0.177 \pm .018$ & $272 \pm 36^{*}$ & nd & nd \\
\hline
\end{tabular}

nd - data not collected. The total $\mathrm{N}$ for each measurement is in parentheses below the mean \pm standard error for that measurement. *Significantly different from the reference site (SC) data from the same year $(p<0.05,2$-tailed t-test). ud - undetectable 
Table 3: Distribution of individual PCB congeners in the carcasses of fish from SC and

$\mathrm{NBH}$

\begin{tabular}{|c|c|c|c|c|c|}
\hline PCB Congener(s) & Position of Chlorines & \multicolumn{2}{|c|}{ PCB (ng/g dry weight) } & \multicolumn{2}{|c|}{ PCB (\% total PCB) } \\
\hline IUPAC \# & & $\mathrm{SC}$ & $\mathrm{NBH}^{+}$ & $\mathrm{SC}$ & $\mathrm{NBH}$ \\
\hline 10,4 & 2,$6 ; 2,2^{\prime}$ & 0 & 0 & 0 & 0 \\
\hline 7 & 2,4 & $\begin{array}{l}0.53 \\
\pm 0.51 \\
\end{array}$ & $\begin{array}{l}76 \\
\pm 42 \\
\end{array}$ & $\begin{array}{l}0.26 \\
\pm 0.13 \\
\end{array}$ & $\begin{array}{l}0.01 \\
\pm 0.00 \\
\end{array}$ \\
\hline 8,5 & $2,4^{\prime} ; 2,3$ & $\begin{array}{l}11.0 \\
\pm 4.68 \\
\end{array}$ & $\begin{array}{l}3147 \\
\pm 826 \\
\end{array}$ & $\begin{array}{l}5.65 \\
\pm 1.73 \\
\end{array}$ & $\begin{array}{l}1.27 \\
\pm 0.32 \\
\end{array}$ \\
\hline 19 & $2,2^{\prime}, 6$ & $\begin{array}{l}0.72 \\
\pm 0.72 \\
\end{array}$ & $\begin{array}{l}172 \\
\pm 86 \\
\end{array}$ & $\begin{array}{l}0.34 \\
\pm 0.18 \\
\end{array}$ & $\begin{array}{l}0.05 \\
\pm 0.02 \\
\end{array}$ \\
\hline 18,17 & $\begin{array}{l}2,2^{\prime}, 5 \\
2,2^{\prime}, 4\end{array}$ & $\begin{array}{l}2.23 \\
\pm 0.61 \\
\end{array}$ & $\begin{array}{l}7127 \\
\pm 1429 \\
\end{array}$ & $\begin{array}{l}1.33 \\
\pm 0.28 \\
\end{array}$ & $\begin{array}{l}2.47 \\
\pm 0.25 \\
\end{array}$ \\
\hline 24,27 & $\begin{array}{l}2,3,6 ; \\
2,3^{\prime}, 6 \\
\end{array}$ & $\begin{array}{l}0.04 \\
\pm 0.04 \\
\end{array}$ & $\begin{array}{l}793 \\
\pm 131 \\
\end{array}$ & $\begin{array}{l}0.02 \\
\pm 0.01 \\
\end{array}$ & $\begin{array}{l}0.30 \\
\pm 0.03^{1} \\
\end{array}$ \\
\hline 16,32 & $\begin{array}{l}2,2,3 ; \\
2,4^{\prime}, 6\end{array}$ & $\begin{array}{l}.02 \\
\pm 0.57 \\
\end{array}$ & $\begin{array}{l}5496 \\
\pm 756 \\
\end{array}$ & $\begin{array}{l}1.33 \\
\pm 0.32 \\
\end{array}$ & $\begin{array}{l}2.02 \\
\pm 0.10 \\
\end{array}$ \\
\hline 26 & $2,3^{\prime}, 5$ & $\begin{array}{l}0.83 \\
\pm 0.41 \\
\end{array}$ & $\begin{array}{l}10205 \\
\pm 1453 \\
\end{array}$ & $\begin{array}{l}0.44 \\
\pm 0.17 \\
\end{array}$ & $\begin{array}{l}3.76 \\
\pm 0.25^{1} \\
\end{array}$ \\
\hline 28,31 & $\begin{array}{l}2,4,4^{\prime} \\
2,4^{\prime}, 5 \\
\end{array}$ & $\begin{array}{l}9.05 \\
\pm 1.74 \\
\end{array}$ & $\begin{array}{l}45271 \\
\pm 7462 \\
\end{array}$ & $\begin{array}{l}5.45 \\
\pm 0.67 \\
\end{array}$ & $\begin{array}{l}16.3 \\
\pm 1.01^{1} \\
\end{array}$ \\
\hline $33,21,53$ & $\begin{array}{l}2^{\prime}, 3,4 ; \\
2,3,4 ; \\
2,2^{\prime}, 5,6^{\prime}\end{array}$ & $\begin{array}{l}1.32 \\
\pm 0.60\end{array}$ & $\begin{array}{l}1952 \\
\pm 248\end{array}$ & $\begin{array}{l}0.70 \\
\pm 0.27\end{array}$ & $\begin{array}{l}0.76 \\
\pm 0.06\end{array}$ \\
\hline 22 & $2,3,4$ & $\begin{array}{l}1.55 \\
\pm 0.76 \\
\end{array}$ & $\begin{array}{l}5449 \\
\pm 727 \\
\end{array}$ & $\begin{array}{l}0.70 \\
\pm 0.27 \\
\end{array}$ & $\begin{array}{l}2.05 \\
\pm 0.11^{1} \\
\end{array}$ \\
\hline 52,43 & $\begin{array}{l}2,2^{\prime}, 5,5^{\prime} \\
2,2^{\prime}, 3,5\end{array}$ & $\begin{array}{l}7.52 \\
\pm 2.09 \\
\end{array}$ & $\begin{array}{l}20509 \\
\pm 2968 \\
\end{array}$ & $\begin{array}{l}4.26 \\
\pm 0.86 \\
\end{array}$ & $\begin{array}{l}7.34 \\
\pm 0.28^{1} \\
\end{array}$ \\
\hline 49 & $2,2^{\prime}, 4,5^{\prime}$ & $\begin{array}{l}4.17 \\
\pm 0.74 \\
\end{array}$ & $\begin{array}{l}19888 \\
\pm 2743 \\
\end{array}$ & $\begin{array}{l}2.47 \\
\pm 0.33 \\
\end{array}$ & $\begin{array}{l}7.23 \\
\pm 0.25 \\
\end{array}$ \\
\hline 47,48 & $\begin{array}{l}2,2^{\prime}, 4,4^{\prime} \\
2,2^{\prime}, 4,5\end{array}$ & $\begin{array}{l}2.63 \\
\pm 0.65 \\
\end{array}$ & $\begin{array}{l}13945 \\
\pm 2105 \\
\end{array}$ & $\begin{array}{l}1.53 \\
\pm 0.26 \\
\end{array}$ & $\begin{array}{l}4.94 \\
\pm 0.15^{1} \\
\end{array}$ \\
\hline 44 & $2,2^{\prime}, 3,5^{\prime}$ & $\begin{array}{l}6.21 \\
\pm 1.65 \\
\end{array}$ & $\begin{array}{l}8424 \\
\pm 1108 \\
\end{array}$ & $\begin{array}{l}3.76 \\
\pm 0.81\end{array}$ & $\begin{array}{l}3.09 \\
\pm 0.10\end{array}$ \\
\hline $37,42^{*}$ & $\begin{array}{l}3,4,4^{\prime} ; \\
2,2^{\prime}, 3,4^{\prime} \\
\end{array}$ & $\begin{array}{l}6.05 \\
\pm 4.55 \\
\end{array}$ & $\begin{array}{l}6315 \\
\pm 995 \\
\end{array}$ & $\begin{array}{l}3.56 \\
\pm 1.45 \\
\end{array}$ & $\begin{array}{l}.39 \\
\pm 0.18 \\
\end{array}$ \\
\hline $41,71,64$ & $\begin{array}{l}2,2^{\prime}, 3,4 ; \\
2,3^{\prime}, 4^{\prime}, 6 \\
2,3,4^{\prime}, 6\end{array}$ & $\begin{array}{l}4.97 \\
\pm 0.93\end{array}$ & $\begin{array}{l}15113 \\
\pm 2172\end{array}$ & $\begin{array}{l}3.03 \\
\pm 0.43\end{array}$ & $\begin{array}{l}5.41 \\
\pm 0.20^{1}\end{array}$ \\
\hline $40^{*}$ & $2,2^{\prime}, 3,3^{\prime}$ & $\begin{array}{l}0.36 \\
\pm 0.17 \\
\end{array}$ & $\begin{array}{l}517 \\
\pm 91 \\
\end{array}$ & $\begin{array}{l}0.19 \\
\pm 0.07 \\
\end{array}$ & $\begin{array}{l}0.21 \\
\pm 0.03 \\
\end{array}$ \\
\hline $70,76^{*}$ & $\begin{array}{l}2,3^{\prime}, 4^{\prime}, 5 \\
2^{\prime}, 3,4,5\end{array}$ & $\begin{array}{l}6.67 \\
\pm 1.09 \\
\end{array}$ & $\begin{array}{l}6772 \\
\pm 965 \\
\end{array}$ & $\begin{array}{l}3.73 \\
\pm 0.33 \\
\end{array}$ & $\begin{array}{l}2.47 \\
\pm 0.10^{1} \\
\end{array}$ \\
\hline 66,95 & $\begin{array}{l}2,3^{\prime}, 4,4^{\prime} ; \\
2,2^{\prime}, 3,5^{\prime}, 6\end{array}$ & $\begin{array}{l}11.0 \\
\pm 1.92 \\
\end{array}$ & $\begin{array}{l}13428 \\
\pm 2895 \\
\end{array}$ & $\begin{array}{l}6.33 \\
\pm 0.58 \\
\end{array}$ & $\begin{array}{l}4.75 \\
\pm 0.56 \\
\end{array}$ \\
\hline
\end{tabular}


Table 3 (cont.): Distribution of individual PCB congeners in the carcasses of fish from $\mathrm{SC}$ and NBH

\begin{tabular}{|c|c|c|c|c|c|}
\hline $\begin{array}{c}\text { PCB } \\
\text { Congener(s) }\end{array}$ & Position of Chlorines & \multicolumn{2}{|c|}{$\begin{array}{l}\text { PCB (ng/g dry } \\
\text { weight) }\end{array}$} & \multicolumn{2}{|c|}{ PCB (\% total PCB) } \\
\hline IUPAC \# & & $\mathrm{SC}$ & $\mathrm{NBH}^{+}$ & $\mathrm{SC}$ & $\mathrm{NBH}$ \\
\hline $92,84,89$ & $\begin{array}{l}2,2^{\prime}, 3,5,5^{\prime} \\
2,2^{\prime}, 3,3^{\prime}, 6^{\prime} \\
2,2^{\prime}, 3,4,6^{\prime}\end{array}$ & $\begin{array}{l}6.13 \\
\pm 1.18\end{array}$ & $\begin{array}{l}6201 \\
\pm 760\end{array}$ & $\begin{array}{l}3.62 \\
\pm 0.41\end{array}$ & $\begin{array}{l}2.31 \\
\pm 0.08^{1}\end{array}$ \\
\hline $101^{*}$ & $2,2^{\prime}, 4,5,5^{\prime}$ & $\begin{array}{l}7.90 \\
\pm 0.98 \\
\end{array}$ & $\begin{array}{l}8673 \\
\pm 1119 \\
\end{array}$ & $\begin{array}{l}4.57 \\
\pm 0.27 \\
\end{array}$ & $\begin{array}{l}3.20 \\
\pm 0.09^{1}\end{array}$ \\
\hline $99^{*}$ & $2,2^{\prime}, 4,4^{\prime}, 5$ & $\begin{array}{l}4.59 \\
\pm 0.80 \\
\end{array}$ & $\begin{array}{l}8538 \\
\pm 992 \\
\end{array}$ & $\begin{array}{l}2.52 \\
\pm 0.26 \\
\end{array}$ & $\begin{array}{l}3.24 \\
\pm 0.11^{1} \\
\end{array}$ \\
\hline 97 & $2,2^{\prime}, 3^{\prime}, 4,5$ & $\begin{array}{l}2.45 \\
\pm 0.53 \\
\end{array}$ & $\begin{array}{l}1650 \\
\pm 252 \\
\end{array}$ & $\begin{array}{l}1.30 \\
\pm 0.20 \\
\end{array}$ & $\begin{array}{l}0.69 \\
\pm 0.09^{1} \\
\end{array}$ \\
\hline $87,81^{*}$ & $\begin{array}{l}2,2^{\prime}, 3,4,5^{\prime} \\
3,4,4^{\prime}, 5\end{array}$ & $\begin{array}{l}3.30 \\
\pm 0.63 \\
\end{array}$ & $\begin{array}{l}1490 \\
\pm 218 \\
\end{array}$ & $\begin{array}{l}1.84 \\
\pm 0.20 \\
\end{array}$ & $\begin{array}{l}0.59 \\
\pm 0.05^{1}\end{array}$ \\
\hline 110,77 & $\begin{array}{l}2,3,3^{\prime}, 4^{\prime}, 6 \\
3,3^{\prime}, 4,4^{\prime}\end{array}$ & $\begin{array}{l}8.06 \\
\pm 1.15 \\
\end{array}$ & $\begin{array}{l}16500 \\
\pm 1900 \\
\end{array}$ & $\begin{array}{l}4.52 \\
\pm 0.33 \\
\end{array}$ & $\begin{array}{l}6.28 \\
\pm 0.21^{1}\end{array}$ \\
\hline 82 & $2,2^{\prime}, 3,3^{\prime}, 4$ & $\begin{array}{l}2.37 \\
\pm 1.71 \\
\end{array}$ & $\begin{array}{l}80 \\
\pm 37 \\
\end{array}$ & $\begin{array}{l}1.14 \\
\pm 0.43 \\
\end{array}$ & $\begin{array}{l}0.03 \\
\pm 0.01 \\
\end{array}$ \\
\hline $151^{*}$ & $2,2^{\prime}, 3,5,5^{\prime}, 6$ & $\begin{array}{l}2.68 \\
\pm 0.37 \\
\end{array}$ & $\begin{array}{l}1960 \\
\pm 281 \\
\end{array}$ & $\begin{array}{l}1.64 \\
\pm 0.17 \\
\end{array}$ & $\begin{array}{l}0.68 \\
\pm 0.05^{1} \\
\end{array}$ \\
\hline $149,118^{*}$ & $\begin{array}{l}2,2^{\prime}, 3,4^{\prime}, 5^{\prime}, 6 \\
2,3^{\prime}, 4,4^{\prime}, 5\end{array}$ & $\begin{array}{l}11.0 \\
\pm 1.90 \\
\end{array}$ & $\begin{array}{l}14300 \\
\pm 2520 \\
\end{array}$ & $\begin{array}{l}6.12 \\
\pm 0.59 \\
\end{array}$ & $\begin{array}{l}5.16 \\
\pm 0.48 \\
\end{array}$ \\
\hline $153,132,105^{*}$ & $\begin{array}{l}2,2^{\prime}, 4,4^{\prime}, 5,5^{\prime} ; \\
2,2^{\prime}, 3,3^{\prime}, 4,6^{\prime} \\
2,3,3^{\prime}, 4,4^{\prime}\end{array}$ & $\begin{array}{l}20.2 \\
\pm 2.60\end{array}$ & $\begin{array}{l}15500 \\
\pm 2100\end{array}$ & $\begin{array}{l}11.5 \\
\pm 0.92\end{array}$ & $\begin{array}{l}5.73 \\
\pm 0.28^{1}\end{array}$ \\
\hline 141 & $2,2^{\prime}, 3,4,5,5^{\prime}$ & $\begin{array}{l}1.32 \\
\pm 0.43 \\
\end{array}$ & $\begin{array}{l}366 \\
\pm 78 \\
\end{array}$ & $\begin{array}{l}0.74 \\
\pm 0.12 \\
\end{array}$ & $\begin{array}{l}0.13 \\
\pm 0.03^{1} \\
\end{array}$ \\
\hline $138,163^{*}$ & $\begin{array}{l}2,2^{\prime}, 3,4,4^{\prime}, 5^{\prime} \\
2,3,3^{\prime}, 4^{\prime}, 5,6 \\
\end{array}$ & $\begin{array}{l}12.2 \\
\pm 1.78 \\
\end{array}$ & $\begin{array}{l}8090 \\
\pm 1100 \\
\end{array}$ & $\begin{array}{l}6.83 \\
\pm 0.61 \\
\end{array}$ & $\begin{array}{l}2.98 \\
\pm 0.15^{1} \\
\end{array}$ \\
\hline 182,187 & $\begin{array}{l}2,2^{\prime}, 3,4,4^{\prime}, 5,6^{\prime} \\
2,2^{\prime}, 3,4^{\prime}, 5,5^{\prime}, 6\end{array}$ & $\begin{array}{l}3.95 \\
\pm 0.84 \\
\end{array}$ & $\begin{array}{r}1430 \\
\pm 213 \\
\end{array}$ & $\begin{array}{l}2.16 \\
\pm 0.32 \\
\end{array}$ & $\begin{array}{l}0.54 \\
\pm 0.05^{1} \\
\end{array}$ \\
\hline $174^{*}$ & $2,2^{\prime}, 3,3^{\prime}, 4,5,6^{\prime}$ & $\begin{array}{l}1.30 \\
\pm 0.23 \\
\end{array}$ & $\begin{array}{l}249 \\
\pm 64 \\
\end{array}$ & $\begin{array}{l}0.84 \\
\pm 0.12 \\
\end{array}$ & $\begin{array}{l}0.10 \\
\pm 0.02^{1} \\
\end{array}$ \\
\hline $202,171,156^{*}$ & $\begin{array}{l}2,2^{\prime}, 3,3^{\prime}, 5,5^{\prime}, 6,6^{\prime} \\
2,2^{\prime}, 3,3^{\prime}, 4,4^{\prime}, 6 \\
2,3,3^{\prime}, 4,4^{\prime}, 5\end{array}$ & $\begin{array}{l}2.10 \\
\pm 1.18\end{array}$ & $\begin{array}{l}686 \\
\pm 171\end{array}$ & $\begin{array}{l}1.05 \\
\pm 0.35\end{array}$ & $\begin{array}{l}0.26 \\
\pm 0.05\end{array}$ \\
\hline 180 & $2,2^{\prime}, 3,4,4^{\prime}, 5,5^{\prime}$ & $\begin{array}{l}3.36 \\
\pm 0.63 \\
\end{array}$ & $\begin{array}{l}1300 \\
\pm 232 \\
\end{array}$ & $\begin{array}{l}1.87 \\
\pm 0.28 \\
\end{array}$ & $\begin{array}{l}0.49 \\
\pm 0.06^{1} \\
\end{array}$ \\
\hline 199 & $2,2^{\prime}, 3,3^{\prime}, 4,5,6,6^{\prime}$ & $\begin{array}{l}0.15 \\
\pm 0.08 \\
\end{array}$ & 0 & $\begin{array}{l}0.09 \\
\pm 0.04 \\
\end{array}$ & 0 \\
\hline 170,190 & $\begin{array}{l}2,2^{\prime}, 3,3^{\prime}, 4,4^{\prime}, 5 \\
2,3,3^{\prime}, 4,4^{\prime}, 5,6 \\
\end{array}$ & $\begin{array}{l}1.38 \\
\pm 0.47 \\
\end{array}$ & $\begin{array}{l}726 \\
\pm 212 \\
\end{array}$ & $\begin{array}{l}0.69 \\
\pm 0.18 \\
\end{array}$ & $\begin{array}{l}0.26 \\
\pm 0.06 \\
\end{array}$ \\
\hline 200 & $2,2^{\prime}, 3,3^{\prime}, 4,5^{\prime}, 6,6^{\prime}$ & $\begin{array}{l}1.13 \\
\pm 0.34 \\
\end{array}$ & $\begin{array}{l}144 \\
\pm 59 \\
\end{array}$ & $\begin{array}{l}0.58 \\
\pm 0.14 \\
\end{array}$ & $\begin{array}{l}0.06^{1} \\
\pm 0.02 \\
\end{array}$ \\
\hline
\end{tabular}


Table 3 (cont.): Distribution of individual PCB congeners in the carcasses of fish from $\mathrm{SC}$ and $\mathrm{NBH}$

\begin{tabular}{|l|l|l|l|l|l|}
\hline $\begin{array}{c}\text { PCB } \\
\text { Congener(s) }\end{array}$ & Position of Chlorines & \multicolumn{2}{c|}{$\begin{array}{c}\text { PCB (ng/g dry } \\
\text { weight) }\end{array}$} & \multicolumn{2}{c|}{ PCB (\% total PCB) } \\
\hline IUPAC \# & & \multicolumn{1}{|c|}{ SC } & \multicolumn{1}{c|}{$\mathrm{NBH}^{+}$} & \multicolumn{1}{c|}{ SC } & \multicolumn{1}{c|}{ NBH } \\
\hline 203,196 & $2,2^{\prime}, 3,4,4^{\prime}, 5,5^{\prime}, 6 ;$ & 1.34 & 298 & 0.72 & 0.14 \\
& $2,2^{\prime}, 3,3^{\prime}, 4,4^{\prime}, 5^{\prime}, 6$ & \pm 0.38 & \pm 111 & \pm 0.17 & $\pm 0.04^{1}$ \\
\hline 195,208 & $2,2^{\prime}, 3,3^{\prime}, 4,4^{\prime}, 5,6 ;$ & 0.43 & 275 & 0.21 & 0.11 \\
& $2,2^{\prime}, 3,3^{\prime}, 4,5,5^{\prime}, 6,6^{\prime}$ & \pm 0.19 & \pm 167 & \pm 0.07 & \pm 0.05 \\
\hline 194 & $2,2^{\prime}, 3,3^{\prime}, 4,4^{\prime}, 5,5^{\prime}$ & 0.24 & 135 & 0.12 & 0.06 \\
& & \pm 0.13 & \pm 66 & \pm 0.04 & \pm 0.02 \\
\hline 206 & $2,2^{\prime}, 3,3^{\prime}, 4^{\prime}, 4^{\prime}, 5,5^{\prime}, 6$ & 0.63 & 414 & 0.30 & 0.17 \\
& & \pm 0.55 & \pm 229 & \pm 0.14 & \pm 0.07 \\
\hline total (ng/g) & & 177 & 272000 & 100 & 100 \\
& & \pm 17.7 & \pm 36100 & & \\
\hline
\end{tabular}

PCB concentrations are the mean \pm standard error of 12 individual carcasses, 6 male and 6 female. * PCB concentrations in male NBH fish are significantly higher than in female NBH fish ( $p<0.05,2$-tailed t-test). There was no significant difference between male and female fish from SC. ${ }^{+}$PCB concentrations (ug/g) were significantly higher $(\mathrm{p}<0.05,2-$ tailed $t$-test) in carcasses from NBH fish than in SC fish for all congeners except for PCBs 10, 4 and 199. ${ }^{1}$ The PCB concentration (\%) was significantly different between sites $(\mathrm{p}<$ $0.05,2$-tailed t-test). 
Table 4: Distribution of individual PCB congeners in the livers of fish from $\mathrm{SC}$ and NBH

\begin{tabular}{|c|c|c|c|c|c|}
\hline & Position of Chlorines & PCB (ng & y weight) & $\mathrm{PCB}$ & otal PCB) \\
\hline IUPAC \# & & $\mathrm{SC}$ & $\mathrm{NBH}^{+}$ & $\mathrm{SC}$ & $\mathrm{NBH}$ \\
\hline 10,4 & 2,$6 ; 2,2^{\prime}$ & $\begin{array}{l}3.33 \\
\pm 3.33 \\
\end{array}$ & $\begin{array}{l}61.5 \\
\pm 46.1 \\
\end{array}$ & $\begin{array}{l}0.24 \\
\pm 0.19 \\
\end{array}$ & $\begin{array}{l}0.005 \\
\pm 0.004 \\
\end{array}$ \\
\hline 7 & 2,4 & 0 & 0 & 0 & 0 \\
\hline 8,5 & $2,4^{\prime} ; 2,3$ & $\begin{array}{l}5.73 \\
\pm 4.64 \\
\end{array}$ & $\begin{array}{l}1701 \\
\pm 262 \\
\end{array}$ & $\begin{array}{l}0.57 \\
\pm 0.34 \\
\end{array}$ & $\begin{array}{l}0.29 \\
\pm 0.07 \\
\end{array}$ \\
\hline 19 & $2,2^{\prime}, 6$ & $\begin{array}{l}5.76 \\
\pm 3.10 \\
\end{array}$ & $\begin{array}{l}1297 \\
\pm 602 \\
\end{array}$ & $\begin{array}{l}0.47 \\
\pm 0.15 \\
\end{array}$ & $\begin{array}{l}0.15 \\
\pm 0.05 \\
\end{array}$ \\
\hline 18,17 & $\begin{array}{l}2,2^{\prime}, 5 \\
2,2^{\prime}, 4\end{array}$ & $\begin{array}{l}0.21 \\
\pm 0.21 \\
\end{array}$ & $\begin{array}{l}7522 \\
\pm 2789 \\
\end{array}$ & $\begin{array}{l}0.02 \\
\pm 0.01 \\
\end{array}$ & $\begin{array}{l}0.92 \\
\pm 0.21^{*}\end{array}$ \\
\hline 24,27 & $\begin{array}{l}2,3,6 \\
2,3^{\prime}, 6 \\
\end{array}$ & $\begin{array}{l}20.2 \\
\pm 10.8 \\
\end{array}$ & $\begin{array}{l}7188 \\
\pm 2560 \\
\end{array}$ & $\begin{array}{l}1.65 \\
\pm 0.54 \\
\end{array}$ & $\begin{array}{l}0.81 \\
\pm 0.14 \\
\end{array}$ \\
\hline 16,32 & $\begin{array}{l}2,2^{\prime}, 3 \\
2,4^{\prime}, 6\end{array}$ & $\begin{array}{l}1.5 \\
\pm 1.5 \\
\end{array}$ & $\begin{array}{l}5502 \\
\pm 2030 \\
\end{array}$ & $\begin{array}{l}0.11 \\
\pm 0.08 \\
\end{array}$ & $\begin{array}{l}0.57 \\
\pm 0.10 \\
\end{array}$ \\
\hline 26 & $2,3^{\prime}, 5$ & $\begin{array}{l}7.16 \\
\pm 1.63 \\
\end{array}$ & $\begin{array}{l}29505 \\
\pm 9562 \\
\end{array}$ & $\begin{array}{l}0.63 \\
\pm 0.05 \\
\end{array}$ & $\begin{array}{l}3.34 \\
\pm 0.34^{*} \\
\end{array}$ \\
\hline 28,31 & $\begin{array}{l}2,4,4^{\prime} \\
2,4^{\prime}, 5 \\
\end{array}$ & $\begin{array}{l}3.33 \\
\pm 3.33 \\
\end{array}$ & $\begin{array}{l}66378 \\
\pm 16416 \\
\end{array}$ & $\begin{array}{l}0.33 \\
\pm 0.25 \\
\end{array}$ & $\begin{array}{l}8.44 \\
\pm 0.23 * \\
\end{array}$ \\
\hline $33,21,53$ & $\begin{array}{l}2^{\prime}, 3,4 ; \\
2,3,4 ; \\
2,2^{\prime}, 5,6^{\prime}\end{array}$ & $\begin{array}{l}6.16 \\
\pm 1.48\end{array}$ & $\begin{array}{l}2928 \\
\pm 792\end{array}$ & $\begin{array}{l}0.54 \\
\pm 0.08\end{array}$ & $\begin{array}{l}0.40 \\
\pm 0.06\end{array}$ \\
\hline 22 & $2,3,4^{\prime}$ & $\begin{array}{l}0.97 \\
\pm 0.97 \\
\end{array}$ & $\begin{array}{l}3121 \\
\pm 1225 \\
\end{array}$ & $\begin{array}{l}0.07 \\
\pm 0.05 \\
\end{array}$ & $\begin{array}{l}0.32 \\
\pm 0.07 \\
\end{array}$ \\
\hline 52,43 & $\begin{array}{l}2,2^{\prime}, 5,5^{\prime} \\
2,2^{\prime}, 3,5\end{array}$ & $\begin{array}{l}2.30 \\
\pm 2.12 \\
\end{array}$ & $\begin{array}{l}45727 \\
\pm 11072 \\
\end{array}$ & $\begin{array}{l}0.17 \\
\pm 0.12 \\
\end{array}$ & $\begin{array}{l}5.84 \\
\pm 0.18^{*} \\
\end{array}$ \\
\hline 49 & $2,2^{\prime}, 4,5^{\prime}$ & $\begin{array}{l}12.0 \\
\pm 6.11 \\
\end{array}$ & $\begin{array}{l}55102 \\
\pm 12933 \\
\end{array}$ & $\begin{array}{l}1.23 \\
\pm 0.47 \\
\end{array}$ & $\begin{array}{l}7.13 \\
\pm 0.29 * \\
\end{array}$ \\
\hline 47,48 & $\begin{array}{l}2,2^{\prime}, 4,4^{\prime} \\
2,2^{\prime}, 4,5\end{array}$ & $\begin{array}{l}13.2 \\
\pm 7.55 \\
\end{array}$ & $\begin{array}{l}34832 \\
\pm 8577 \\
\end{array}$ & $\begin{array}{l}1.33 \\
\pm 0.51 \\
\end{array}$ & $\begin{array}{l}4.44 \\
\pm 0.19^{*} \\
\end{array}$ \\
\hline 44 & $2,2^{\prime}, 3,5^{\prime}$ & $\begin{array}{l}4.60 \\
\pm 4.60 \\
\end{array}$ & $\begin{array}{l}16208 \\
\pm 3778 \\
\end{array}$ & $\begin{array}{l}0.49 \\
\pm 0.37 \\
\end{array}$ & $\begin{array}{l}2.10 \\
\pm 0.09 \\
\end{array}$ \\
\hline 37,42 & $\begin{array}{l}3,4,4^{\prime} ; \\
2,2^{\prime}, 3,4^{\prime} \\
\end{array}$ & 0 & 0 & 0 & 0 \\
\hline $41,71,64$ & $\begin{array}{l}2,2^{\prime}, 3,4 ; \\
2,3^{\prime}, 4^{\prime}, 6 ; \\
2,3,4^{\prime}, 6\end{array}$ & $\begin{array}{l}11.6 \\
\pm 3.35\end{array}$ & $\begin{array}{l}39635 \\
\pm 10234\end{array}$ & $\begin{array}{l}1.14 \\
\pm 0.29\end{array}$ & $\begin{array}{l}4.96 \\
\pm 0.10^{*}\end{array}$ \\
\hline 40 & $2,2^{\prime}, 3,3^{\prime}$ & $\begin{array}{l}3.75 \\
\pm 3.75 \\
\end{array}$ & $\begin{array}{l}697 \\
\pm 523 \\
\end{array}$ & $\begin{array}{l}0.27 \\
\pm 0.21 \\
\end{array}$ & $\begin{array}{l}0.05 \\
\pm 0.04 \\
\end{array}$ \\
\hline 70,76 & $\begin{array}{l}2,3^{\prime}, 4^{\prime}, 5 ; \\
2^{\prime}, 3,4,5\end{array}$ & $\begin{array}{l}7.59 \\
\pm 1.89 \\
\end{array}$ & $\begin{array}{l}6882 \\
\pm 1772 \\
\end{array}$ & $\begin{array}{l}0.67 \\
\pm 0.07 \\
\end{array}$ & $\begin{array}{l}0.86 \\
\pm 0.01 \\
\end{array}$ \\
\hline 66,95 & $\begin{array}{l}2,3^{\prime}, 4,4^{\prime} ; \\
2,2^{\prime}, 3,5^{\prime}, 6\end{array}$ & $\begin{array}{l}7.16 \\
\pm 3.01 \\
\end{array}$ & $\begin{array}{l}34440 \\
\pm 8611 \\
\end{array}$ & $\begin{array}{l}0.72 \\
\pm 0.23 \\
\end{array}$ & $\begin{array}{l}4.35 \\
\pm 0.14^{*} \\
\end{array}$ \\
\hline
\end{tabular}


Table 4 (cont.): Distribution of individual PCB congeners in the livers of fish from SC and $\mathrm{NBH}$

\begin{tabular}{|c|c|c|c|c|c|}
\hline \multirow{2}{*}{$\begin{array}{c}\text { PCB } \\
\text { Congener(s) } \\
\text { IUPAC \# } \\
\end{array}$} & \multirow[t]{2}{*}{ Position of Chlorines } & \multicolumn{2}{|c|}{ PCB (ng/g dry weight) } & \multicolumn{2}{|c|}{ PCB (\% total PCB) } \\
\hline & & $\mathrm{SC}$ & $\mathrm{NBH}^{+}$ & $\mathrm{SC}$ & $\mathrm{NBH}$ \\
\hline $92,84,89$ & $\begin{array}{l}2,2^{\prime}, 3,5,5^{\prime} \\
2,2^{\prime}, 3,3^{\prime}, 6^{\prime} \\
2,2^{\prime}, 3,4,6^{\prime}\end{array}$ & $\begin{array}{l}7.24 \\
\pm 5.13\end{array}$ & $\begin{array}{l}10430 \\
\pm 3714\end{array}$ & $\begin{array}{l}0.58 \\
\pm 0.26\end{array}$ & $\begin{array}{l}1.10 \\
\pm 0.20\end{array}$ \\
\hline 101 & $2,2^{\prime}, 4,5,5^{\prime}$ & $\begin{array}{l}18.1 \\
\pm 9.01 \\
\end{array}$ & $\begin{array}{l}42554 \\
\pm 12173 \\
\end{array}$ & $\begin{array}{l}1.72 \\
\pm 0.74 \\
\end{array}$ & $\begin{array}{l}5.07 \\
\pm 0.47 \\
\end{array}$ \\
\hline 99 & $2,2^{\prime}, 4,4^{\prime}, 5$ & $\begin{array}{l}45.4 \\
\pm 22.8 \\
\end{array}$ & $\begin{array}{l}65000 \\
\pm 17300 \\
\end{array}$ & $\begin{array}{l}4.62 \\
\pm 1.78 \\
\end{array}$ & $\begin{array}{l}8.01 \\
\pm 0.23 \\
\end{array}$ \\
\hline 97 & $2,2^{\prime}, 3^{\prime}, 4,5$ & $\begin{array}{l}1.03 \\
\pm 1.03 \\
\end{array}$ & $\begin{array}{l}19900 \\
\pm 7310 \\
\end{array}$ & $\begin{array}{l}0.07 \\
\pm 0.06\end{array}$ & $\begin{array}{l}2.07 \\
\pm 0.35^{*} \\
\end{array}$ \\
\hline 87,81 & $\begin{array}{l}2,2^{\prime}, 3,4,5^{\prime} \\
3,4,4^{\prime}, 5\end{array}$ & $\begin{array}{l}10.4 \\
\pm 3.88 \\
\end{array}$ & $\begin{array}{l}9000 \\
\pm 2240 \\
\end{array}$ & $\begin{array}{l}0.98 \\
\pm 0.31\end{array}$ & $\begin{array}{l}1.14 \\
\pm 0.05 \\
\end{array}$ \\
\hline 110,77 & $\begin{array}{l}2,3,3^{\prime}, 4^{\prime}, 6 \\
3,3^{\prime}, 4,4^{\prime}\end{array}$ & $\begin{array}{l}1.89 \\
\pm 1.89 \\
\end{array}$ & $\begin{array}{l}75500 \\
\pm 20200\end{array}$ & $\begin{array}{l}0.20 \\
\pm 0.15\end{array}$ & $\begin{array}{l}9.31 \\
\pm 0.18^{*}\end{array}$ \\
\hline 82 & $2,2^{\prime}, 3,3^{\prime}, 4$ & $\begin{array}{l}24.8 \\
\pm 8.87 \\
\end{array}$ & $\begin{array}{l}2500 \\
\pm 558 \\
\end{array}$ & $\begin{array}{l}2.43 \\
\pm 0.79\end{array}$ & $\begin{array}{l}0.33 \\
\pm 0.03 \\
\end{array}$ \\
\hline 151 & $2,2^{\prime}, 3,5,5^{\prime}, 6$ & $\begin{array}{l}9.65 \\
\pm 4.82 \\
\end{array}$ & $\begin{array}{l}7050 \\
\pm 1630 \\
\end{array}$ & $\begin{array}{l}0.98 \\
\pm 0.38 \\
\end{array}$ & $\begin{array}{l}0.92 \\
\pm 0.04 \\
\end{array}$ \\
\hline 149,118 & $\begin{array}{l}2,2^{\prime}, 3,4^{\prime}, 5^{\prime}, 6 \\
2,3^{\prime}, 4,4^{\prime}, 5\end{array}$ & $\begin{array}{l}37.5 \\
\pm 7.06 \\
\end{array}$ & $\begin{array}{l}66900 \\
\pm 17800 \\
\end{array}$ & $\begin{array}{l}3.33 \\
\pm 0.17 \\
\end{array}$ & $\begin{array}{l}8.28 \\
\pm 0.08 \\
\end{array}$ \\
\hline $153,132,105$ & $\begin{array}{l}2,2^{\prime}, 4,4^{\prime}, 5,5^{\prime} ; \\
2,2^{\prime}, 3,3^{\prime}, 4,6^{\prime} ; 2,3,3^{\prime}, 4,4^{\prime}\end{array}$ & $\begin{array}{l}93.2 \\
\pm 2.05 \\
\end{array}$ & $\begin{array}{l}41600 \\
\pm 11100 \\
\end{array}$ & $\begin{array}{l}8.62 \\
\pm 0.76 \\
\end{array}$ & $\begin{array}{l}5.14 \\
\pm 0.15 \\
\end{array}$ \\
\hline 141 & $2,2^{\prime}, 3,4,5,5^{\prime}$ & $\begin{array}{l}35.8 \\
\pm 16.5 \\
\end{array}$ & $\begin{array}{l}1960 \\
\pm 1100 \\
\end{array}$ & $\begin{array}{l}3.01 \\
\pm 0.97 \\
\end{array}$ & $\begin{array}{l}0.24 \\
\pm 0.12 \\
\end{array}$ \\
\hline 138,163 & $\begin{array}{l}2,2^{\prime}, 3,4,4^{\prime}, 5^{\prime} \\
2,3,3^{\prime}, 4^{\prime}, 5,6 \\
\end{array}$ & $\begin{array}{l}100 \\
\pm 4.35 \\
\end{array}$ & $\begin{array}{l}33200 \\
\pm 9320 \\
\end{array}$ & $\begin{array}{l}9.27 \\
\pm 0.88 \\
\end{array}$ & $\begin{array}{l}4.03 \\
\pm 0.14^{*} \\
\end{array}$ \\
\hline 182,187 & $\begin{array}{l}2,2^{\prime}, 3,4,4^{\prime}, 5,6^{\prime} \\
2,2^{\prime}, 3,4^{\prime}, 5,5^{\prime}, 6^{\prime}\end{array}$ & $\begin{array}{l}49.8 \\
\pm 0.72 \\
\end{array}$ & $\begin{array}{l}8390 \\
\pm 2340 \\
\end{array}$ & $\begin{array}{l}4.58 \\
\pm 0.36\end{array}$ & $\begin{array}{l}1.02 \\
\pm 0.03 * \\
\end{array}$ \\
\hline 174 & $2,2^{\prime}, 3,3^{\prime}, 4,5,6^{\prime}$ & $\begin{array}{l}22.9 \\
\pm 8.75 \\
\end{array}$ & $\begin{array}{l}2850 \\
\pm 800 \\
\end{array}$ & $\begin{array}{l}1.94 \\
\pm 0.38 \\
\end{array}$ & $\begin{array}{l}0.34 \\
\pm 0.03 \\
\end{array}$ \\
\hline $202,171,156$ & $\begin{array}{l}2,2^{\prime}, 3,3^{\prime}, 5,5^{\prime}, 6,6^{\prime} ; \\
2,2^{\prime}, 3,3^{\prime}, 4,4^{\prime}, 6 ; \\
2,3,3^{\prime}, 4,4^{\prime}, 5\end{array}$ & $\begin{array}{l}50.7 \\
\pm 5.46\end{array}$ & $\begin{array}{l}10300 \\
\pm 2280\end{array}$ & $\begin{array}{l}4.57 \\
\pm 0.05\end{array}$ & $\begin{array}{l}1.37 \\
\pm 0.17\end{array}$ \\
\hline 180 & $2,2^{\prime}, 3,4,4^{\prime}, 5,5^{\prime}$ & $\begin{array}{l}53.1 \\
\pm 3.99 \\
\end{array}$ & $\begin{array}{l}12000 \\
\pm 3100 \\
\end{array}$ & $\begin{array}{l}4.82 \\
\pm 0.16\end{array}$ & $\begin{array}{l}1.50 \\
\pm 0.10^{*} \\
\end{array}$ \\
\hline 199 & $2,2^{\prime}, 3,3^{\prime}, 4,5,6,6^{\prime}$ & $\begin{array}{l}8.33 \\
\pm 6.03 \\
\end{array}$ & $\begin{array}{l}519 \\
\pm 73 \\
\end{array}$ & $\begin{array}{l}0.65 \\
\pm 0.32 \\
\end{array}$ & $\begin{array}{l}0.08 \\
\pm 0.02 * \\
\end{array}$ \\
\hline 170,190 & $\begin{array}{l}2,2^{\prime}, 3,3^{\prime}, 4,4^{\prime}, 5 \\
2,3,3^{\prime}, 4,4^{\prime}, 5,6\end{array}$ & $\begin{array}{l}15.11 \\
\pm 6.44 \\
\end{array}$ & $\begin{array}{l}5160 \\
\pm 1660 \\
\end{array}$ & $\begin{array}{l}1.27 \\
\pm 0.28 \\
\end{array}$ & $\begin{array}{l}0.58 \\
\pm 0.06 \\
\end{array}$ \\
\hline 200 & $2,2^{\prime}, 3,3^{\prime}, 4,5^{\prime}, 6,6^{\prime}$ & $\begin{array}{l}314 \\
\pm 90.5 \\
\end{array}$ & $\begin{array}{l}20430 \\
\pm 4430 \\
\end{array}$ & $\begin{array}{l}27.2 \\
\pm 3.34 \\
\end{array}$ & $\begin{array}{l}2.73 \\
\pm 0.30^{*} \\
\end{array}$ \\
\hline
\end{tabular}


Table 4 (cont.): Distribution of individual PCB congeners in the livers of fish from SC and $\mathrm{NBH}$

\begin{tabular}{|c|c|c|c|c|c|}
\hline PCB & Position of Chlorines & \multicolumn{2}{|c|}{ PCB (ng/g dry weight) } & \multicolumn{2}{|c|}{ PCB (\% total PCB) } \\
\hline IUPAC \# & & $\mathrm{SC}$ & $\mathrm{NBH}^{+}$ & $\mathrm{SC}$ & NBH \\
\hline 203,196 & $\begin{array}{l}2,2^{\prime}, 3,4,4^{\prime}, 5,5^{\prime}, 6 \\
2,2^{\prime}, 3,3^{\prime}, 4,4^{\prime}, 5^{\prime}, 6\end{array}$ & $\begin{array}{l}48.6 \\
\pm 29.0 \\
\end{array}$ & $\begin{array}{l}2630 \\
\pm 619 \\
\end{array}$ & $\begin{array}{l}3.90 \\
\pm 1.46 \\
\end{array}$ & $\begin{array}{l}0.34 \\
\pm 0.03 \\
\end{array}$ \\
\hline 195,208 & $\begin{array}{l}2,2^{\prime}, 3,3^{\prime}, 4,4^{\prime}, 5,6 \\
2,2^{2}, 3,3^{\prime} 4,5,5,5^{\prime}, 6,6^{\prime}\end{array}$ & $\begin{array}{l}2.78 \\
\pm 2.78 \\
\end{array}$ & $\begin{array}{l}2820 \\
\pm 1057 \\
\end{array}$ & $\begin{array}{l}0.29 \\
\pm 0.23 \\
\end{array}$ & $\begin{array}{l}0.29 \\
\pm 0.05 \\
\end{array}$ \\
\hline 194 & $2,2^{\prime}, 3,3^{\prime}, 4,4^{\prime}, 5,5^{\prime}$ & $\begin{array}{l}32.0 \\
\pm 6.05 \\
\end{array}$ & $\begin{array}{l}5570 \\
\pm 1496 \\
\end{array}$ & $\begin{array}{l}3.06 \\
\pm 0.62 \\
\end{array}$ & $\begin{array}{l}0.68 \\
\pm 0.05 \\
\end{array}$ \\
\hline 206 & $2,2^{\prime}, 3,3^{\prime}, 4,4^{\prime}, 5,5,5^{\prime}, 6$ & $\begin{array}{l}14.3 \\
\pm 1.74 \\
\end{array}$ & $\begin{array}{l}3670 \\
\pm 951 \\
\end{array}$ & $\begin{array}{l}1.31 \\
\pm 0.16 \\
\end{array}$ & $\begin{array}{l}0.46 \\
\pm 0.07 \\
\end{array}$ \\
\hline total (ng/g) & & $\begin{array}{l}1110 \\
\pm 132\end{array}$ & $\begin{array}{l}809000 \\
\pm 215000 \\
\end{array}$ & 100 & 100 \\
\hline
\end{tabular}

PCB concentrations are the mean \pm standard error of $3(\mathrm{SC})$ or $4(\mathrm{NBH})$ liver pools (see methods) + PCB concentrations (ug / g) are significantly higher in livers from NBH fish compared to SC fish for all congeners except PCBs 7 and 37, 42 ( $p<0.05,2$-tailed $t$-test). * PCB concentrations (\%) are significantly different between sites $(p<0.05,2$-tailed ttest) 
Fig 2-1: The difference in the distribution of $\mathrm{PCB}$ congeners in the carcass and liver of SC and NBH Fundulus. PCB congeners were converted to a percent of total PCB concentration then the mean percent of each congener in NBH carcasses (A) or livers (B) was subtracted from the percent of the same congener in SC carcasses (A) or livers (B). 

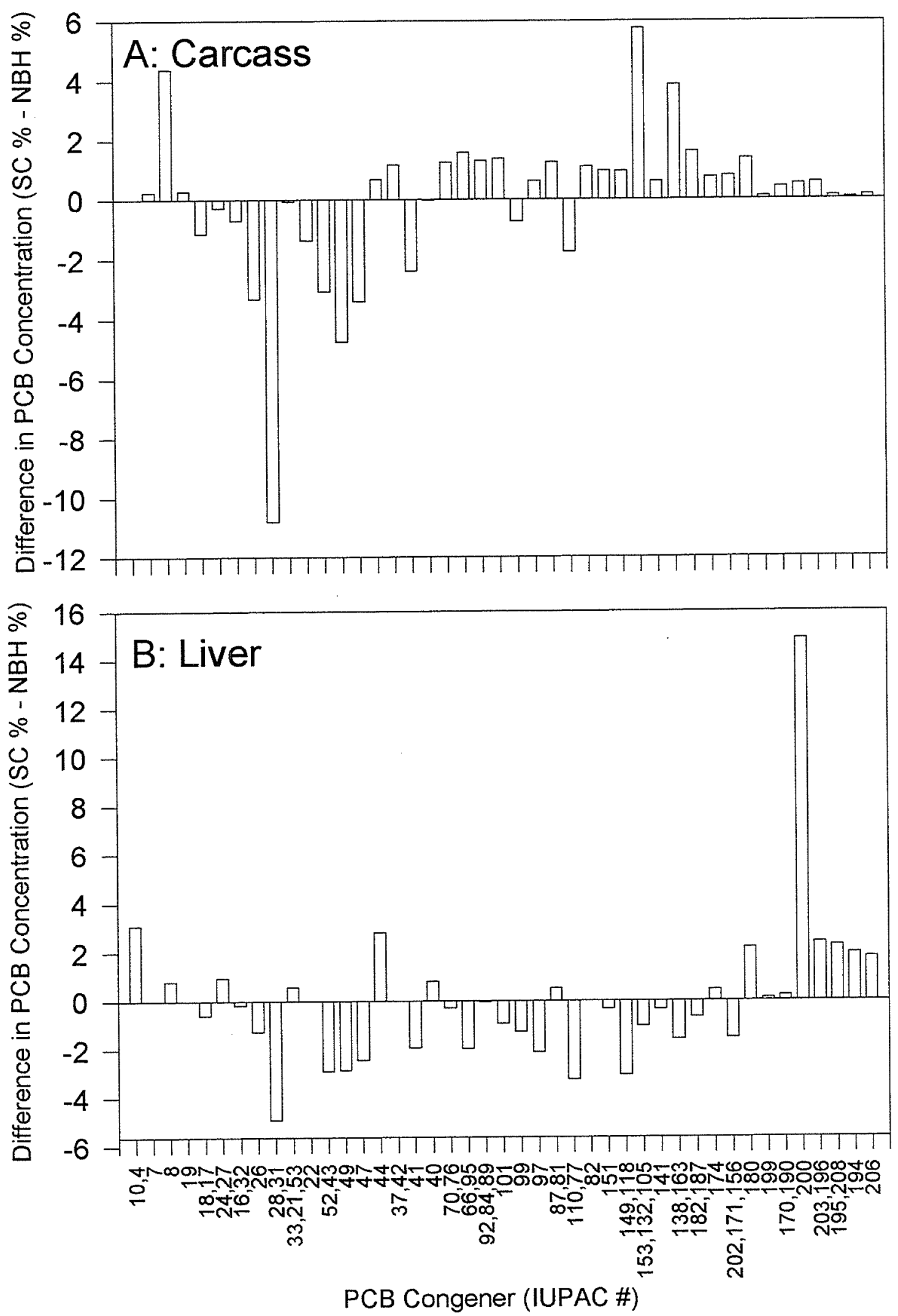
Fig. 2-2: PCB congener distribution in the liver and carcass of $\mathrm{SC}(\mathrm{A})$ and $\mathrm{NBH}(\mathrm{B})$ fish. Each bar represents the percent contribution of all of the congeners measured with the indicated number of chlorines. Peaks which contained congeners with differing numbers of chlorines were not included in this analysis. 

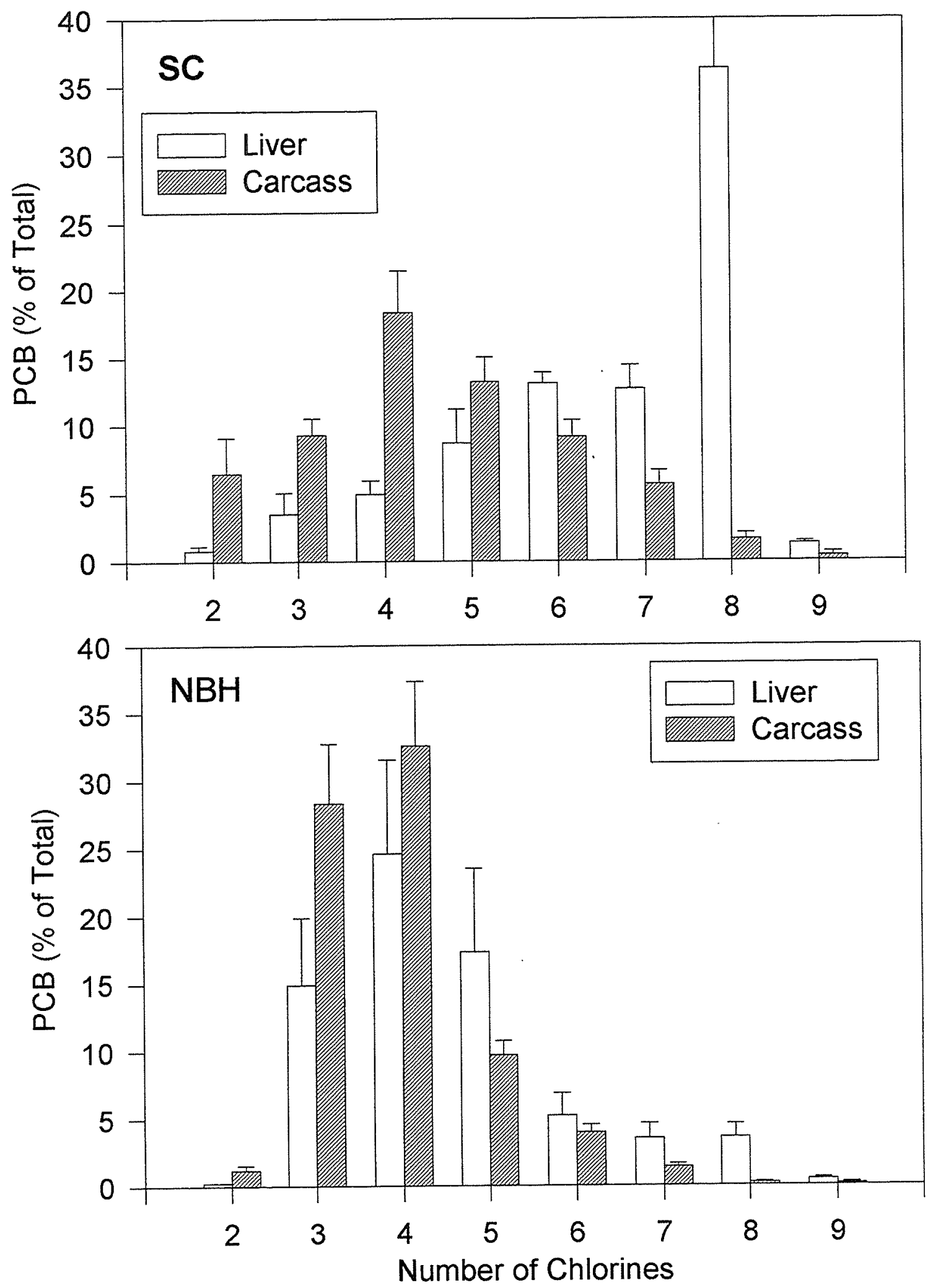
Fig. 2-3: EROD activity in PLHC cells treated with serial dilutions of soxhlet extracts of livers from $\mathrm{NBH}$ or SC fish $(0.00005-0.5 \%)$ or with serial dilutions of TCDD $(0.001$ 30nM). Circles represent TCDD-treated cells, squares represent cells treated with extracts from NBH livers, triangles represent cells treated with extracts from SC livers. Symbols are the means \pm the standard error of 3 replicate wells. 


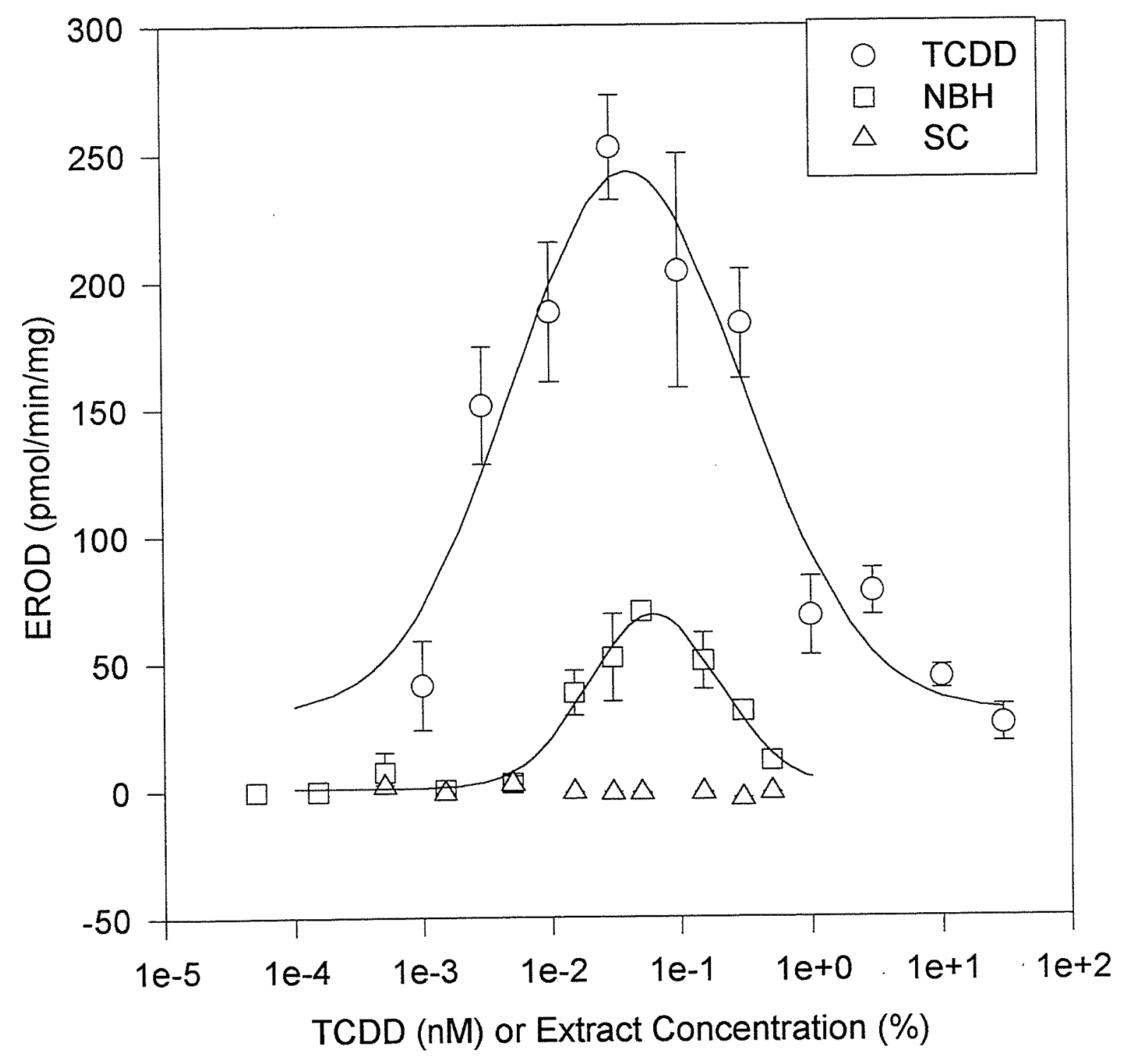




\section{CYP1A1:}

Rates of hepatic microsomal EROD activity were higher in freshly caught fish from $\mathrm{SC}$ than in fish from NBH. This was the case for fish caught in 1994 (Fig. 2-4A) and in 1997 (Table. 4) despite differences in the EROD values between years and regardless of how EROD activity was normalized (either to $\mathrm{mg}$ of total microsomal protein or to $\mathrm{g}$ of tissue weight, Table 4). Hepatic microsomal EROD values in control and TCDF treated Fundulus (Ch. 3) are shown in Figure 2-5A, for comparison; hepatic EROD activity in NBH fish is more similar to control than induced EROD values. The highest hepatic EROD activities in the SC fish were less than half of the activity found in the livers of TCDF treated fish, suggesting that while the hepatic EROD activity in SC Fundulus may be induced it is not maximally induced. There was no consistent trend to the change in EROD activity over the first 90 days after collection (Fig. 2-4A); i.e. there was no significant difference between EROD activity in the livers of fish 1 day after collection and 90 days after collection. However, EROD activity was higher in samples from SC fish than in $\mathrm{NBH}$ fish at each time.

In contrast to the EROD results, there was no consistent difference in hepatic CYP1Al protein between sites (Fig. 2-4B). Again, there was no significant difference between CYP1A1 protein content in the livers of fish 1 day after collection compared to 90 days after collection. In 1997, CYP1A1 protein in the livers was examined using IHC (Fig 2-5 C \& D). As in 1994, there were only low levels of CYP1A1 protein detected in the livers of fish from either site.

In 1997, CYP1A1 messenger RNA (mRNA) was also examined. No livers from $\mathrm{SC}$ fish were available for direct comparison to NBH fish, therefore CYP1A1 mRNA in livers from NBH fish was compared to CYP1A1 mRNA levels control and TCDF treated fish. This comparison revealed that CYP1A1 mRNA levels in livers from NBH fish was more similar to the expression seen in control fish than in fish treated with TCDF (Fig. 26). 
To determine if the differences observed in hepatic CYP1A1 expression were systemic or limited to the liver, EROD activity was examined in pools (4 fish per pool) of heart, kidney, intestine and gill from fish collected in 1997 (Table 5). Microsomal EROD rates were much lower in these extrahepatic tissues than in the liver. EROD activity was significantly elevated in NBH kidney microsomes compared to SC kidney microsomes ( $\mathrm{p}$ $<0.05$ ). There were no significant differences in any of the other extrahepatic tissues

Using IHC staining, CYP1Al protein was also examined in the extrahepatic tissues of freshly caught fish. Little CYP1A1 protein was detected in the heart, spleen, intestine, gill, or gonad of fish from either site (Fig. 2-6 A \& B and Table 6). Moderate levels of CYP1A1 protein (IHC scores from 5-10) were detected in the kidney proximal tubules of fish from both sites (Fig. 2-6 E \& F). There were no consistent differences in CYP1A1 protein levels between sites or sexes in any of the extrahepatic tissues examined.

CYP1A1 mRNA content was examined in the same suite of tissues. CYP1A1 mRNA expression in most of the extrahepatic tissues was barely detectable (Fig. 2-6). CYP1A1 mRNA levels in the hearts from SC fish were significantly higher than the levels in hearts from NBH fish. There was no significant difference in the CYP1A1 mRNA levels between any of the other extrahepatic tissues from NBH fish compared to SC fish. 
Table 5: Hepatic and extrahepatic EROD activity based on microsomal protein (mg) or tissue weight (g) in SC and NBH Fundulus.

\begin{tabular}{|l|l|l|l|l|}
\hline & \multicolumn{2}{|l|}{$\begin{array}{l}\text { EROD (pmol / min / mg total } \\
\text { protein) }\end{array}$} & $\begin{array}{l}\text { EROD (pmol / min / g tissue } \\
\text { weight) }\end{array}$ \\
\hline Site/Tissue & SC & NBH & SC & NBH \\
\hline Liver (1994, day1) & $354 \pm 52$ & $51.0 \pm 17.1^{*}$ & $1860 \pm 68$ & $302 \pm 137$ \\
\hline$(1994$, day 8) & $514 \pm 37$ & $38.6 \pm 11.6^{*}$ & $3080 \pm 1323$ & $154 \pm 68$ \\
\hline$(1994$, day 25) & $170 \pm 27$ & $57.2 \pm 20.8$ & $1540 \pm 263$ & $265 \pm 144$ \\
\hline$(1994$, day 90) & $268 \pm 46$ & $109 \pm 18^{*}$ & $2110 \pm 796$ & $1050 \pm 302$ \\
\hline Liver (1997) & $642 \pm 68$ & $326 \pm 25.1^{*}$ & $595 \pm 192$ & $216 \pm 55.7^{*}$ \\
\hline Heart (1997) & $76.0 \pm 23.6$ & $22.4 \pm 12.9$ & $19.3 \pm 4.2$ & $6.1 \pm 3.2$ \\
\hline Kidney (1997) & $23.0 \pm 4.8$ & $107 \pm 25.8^{*}$ & $20.2 \pm 4.1$ & $50.2 \pm 18.2^{*}$ \\
\hline Gill (1997) & $1.8 \pm 0.4$ & $4.4 \pm 1.3$ & $15.5 \pm 3.2$ & $16.0 \pm 5.6$ \\
\hline Intestine (1997) & $5.9 \pm 3.8$ & $13.9 \pm 3.4$ & $139 \pm 75.9$ & $150 \pm 40.5$ \\
\hline
\end{tabular}

The Ns for these data are presented in Table 1. * Significantly different from the activity in the same tissue in $\mathrm{SC}$ fish 
Table 6: CYP1Al protein levels as determined by IHC in SC and NBH fish collected in 1997 one day after collection

\begin{tabular}{|l|c|c|}
\hline & SC & NBH \\
\hline Liver & Int. x Occ. & Int. x Occ. \\
\hline hepatocytes & $1.3 \pm 0.7(6)$ & $2.6 \pm 0.5(6)$ \\
\hline vascular endothelium & $0.5 \pm 0.5(6)$ & $0(6)$ \\
\hline Gill & & \\
\hline epithelium & $1.2 \pm 0.7(5)$ & $2.7 \pm 0.4(6)$ \\
\hline vascular endothelium & $3.4 \pm 0.9(5)$ & $0(6)$ \\
\hline Kidney & & \\
\hline proximal tubules & $7.3 \pm 0.5(6)$ & $8.5 \pm 0.6(6)$ \\
\hline distal tubules & $0.8 \pm 0.4(6)$ & $1.5 \pm 0.4(6)$ \\
\hline vascular endothelium & $0(6)$ & $0(6)$ \\
\hline Spleen & & $0(6)$ \\
\hline parenchyma & $0(5)$ & $0(6)$ \\
\hline vascular endothelium & $0.2 \pm 0.2(5)$ & \\
\hline Intestine & & $2.3 \pm 0.4(6)$ \\
\hline epithelium & $2.3 \pm 0.3(6)$ & $0(6)$ \\
\hline vascular endothelium & $1.5 \pm 0.6(6)$ & \\
\hline Gonad & & $0(6)$ \\
\hline gametes & $0(4)$ & $0(5)$ \\
\hline vascular endothelium & $1.5 \pm 0.9(4)$ & \\
\hline Heart & & $3.5 \pm 0.5(2)$ \\
\hline atrial endothelium & $9.0(1)$ & $3.8 \pm 0.9(5)$ \\
\hline ventricle endothelium & $3.1 \pm 0.3(6)$ & \\
\hline
\end{tabular}

*Tissues were scored on two scales intensity (Int.) and occurrence (Occ.) resulting in final scores that could range from 0-15 (see methods). The number of individuals scored for each cell type are shown in parentheses after each score. 
Fig. 2-4: Hepatic EROD activity (A) and CYP1A1 protein (B) in NBH and SC fish collected in 1994 1, 8, 25, and 90 days after collection. (A) Hepatic EROD activity in control and TCDF-treated SC fish are shown for comparison for a complete description of these data see chapter 3 . 

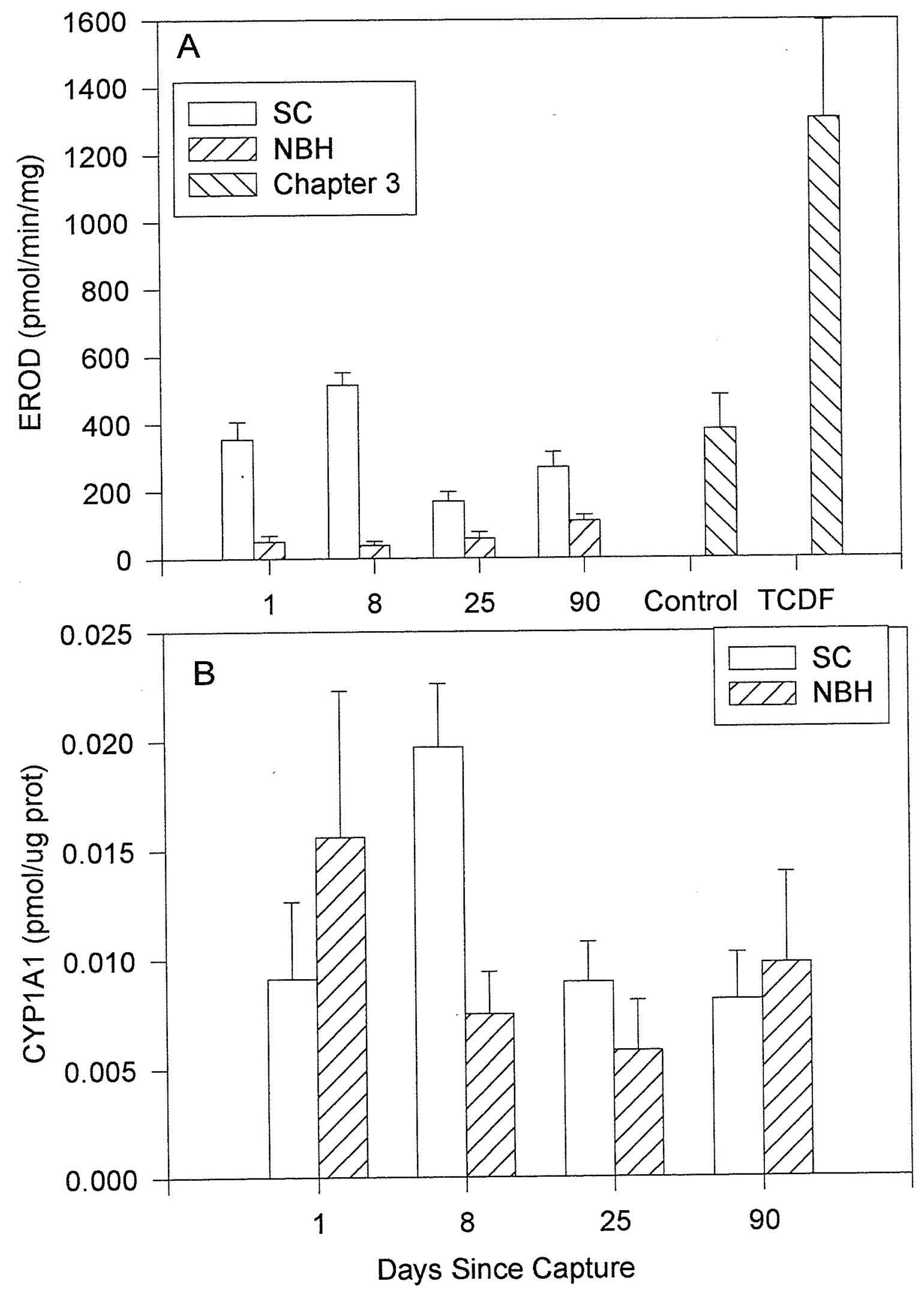
Fig. 2-5: Immunohistochemical sections stained for CYP1A1 in NBH and SC fish collected in 1997 one day after collection. (A) SC atrium, (B) NBH atrium, (C) SC liver (D) NBH liver (E) SC kidney (F) NBH kidney. The arrows indicate the proximal tubules. The NBH liver sample shows an extensive parasitic infestation which was present in many of the samples. Staining intensity is proportional to the amount of CYP1A1 protein present. 

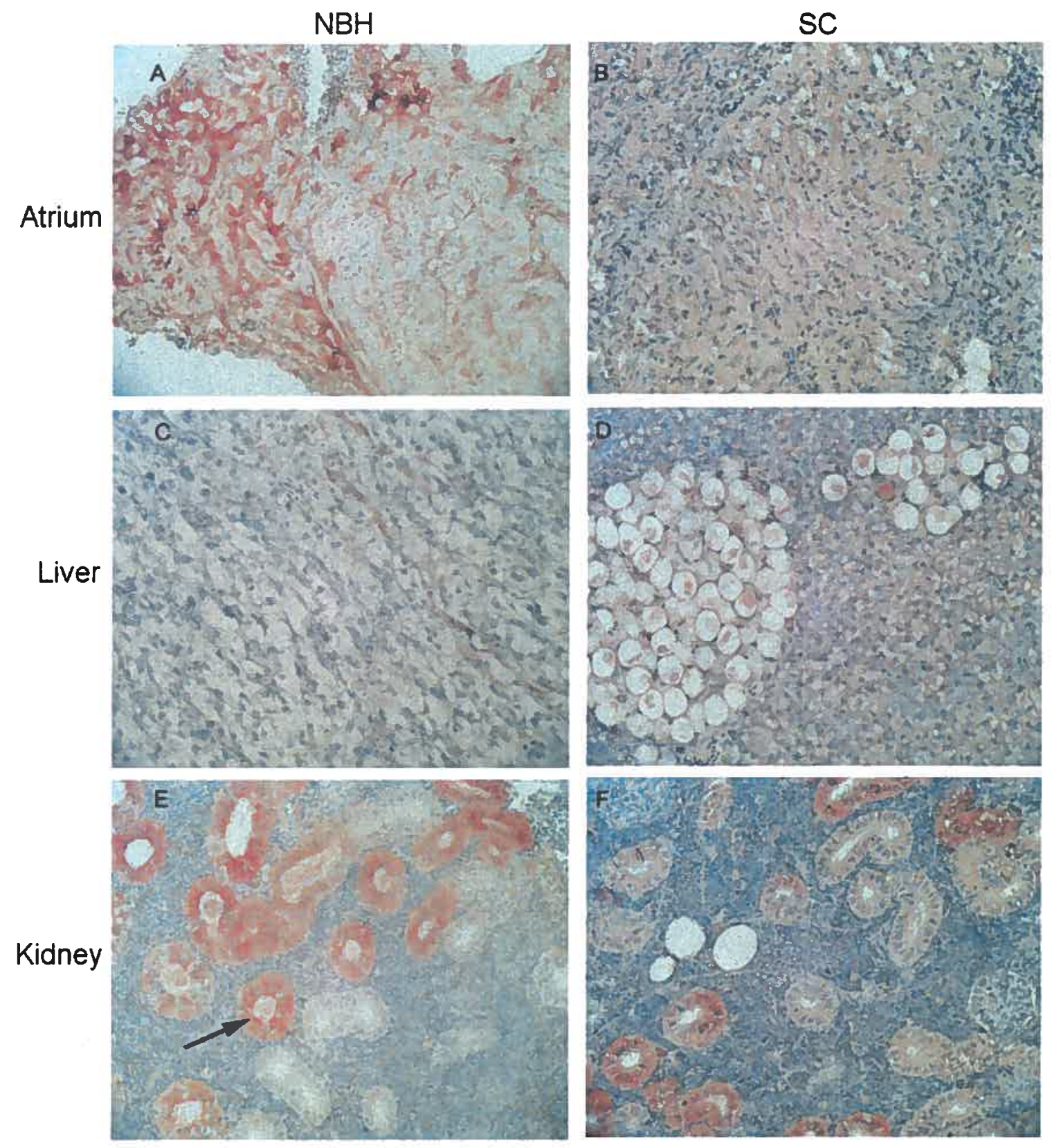
Fig. 2-6: CYP1A1mRNA levels in 10 ug of RNA prepared from pools of kidney, intestine, gonad, gill, heart, spleen, or liver from SC and NBH fish collected in 1997 one day after collection. Also shown are hepatic CYP1A1mRNA levels in 10 ug of RNA from control $\mathrm{SC}$ and $\mathrm{NBH}$ and from TCDF treated SC fish. Samples were normalized to positive control CYP1A1 mRNA (10 ug of gill RNA from TCDF treated killifish from SC (Ch. 3)). Samples were analyzed using a dotblot technique (see methods). $N D=$ not determined. * CYP1A1 mRNA was significantly higher in SC fish than in NBH fish ( $\mathrm{p}<$ $0.05,2$-tailed t-test) 


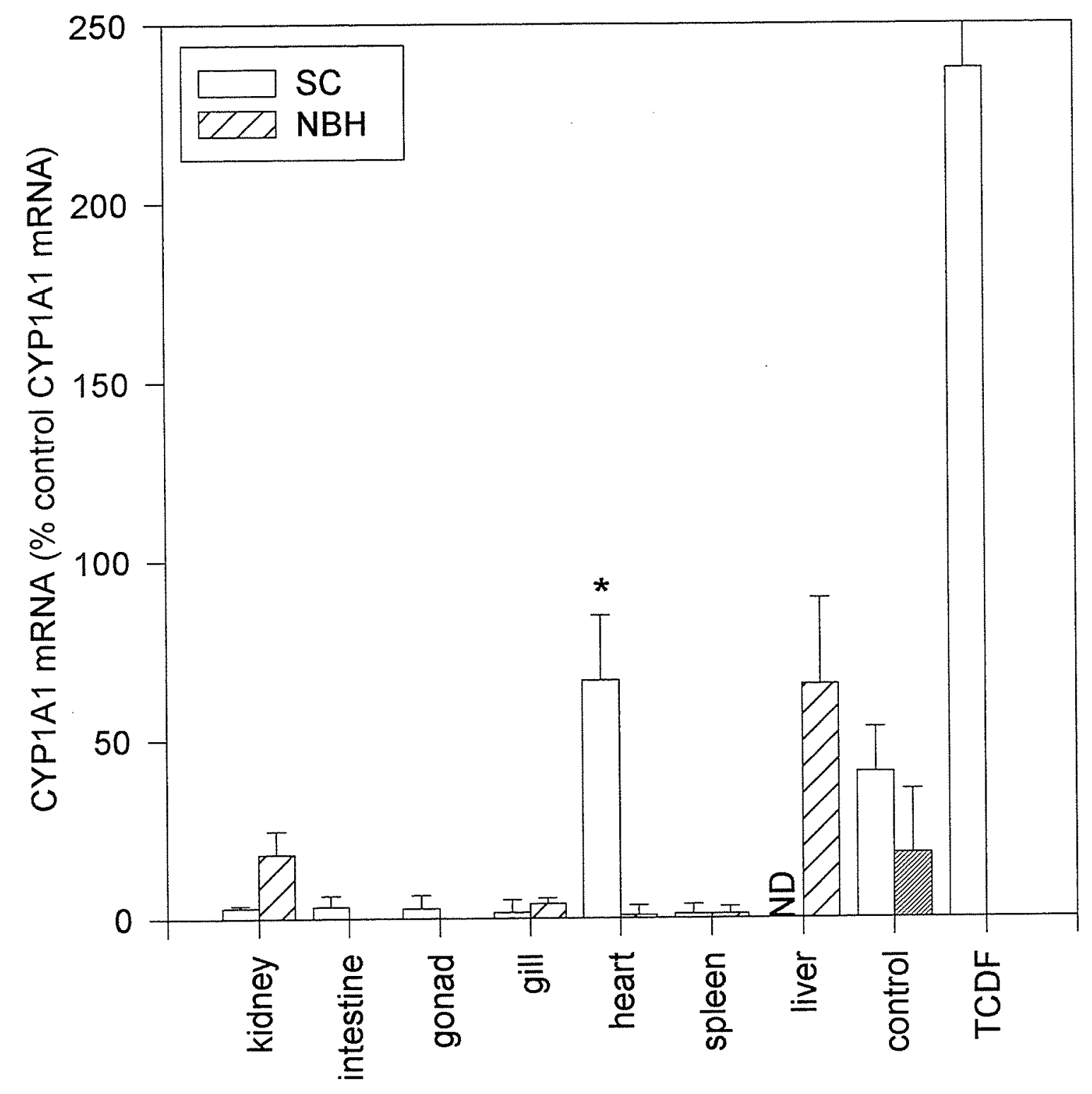


GST:

Hepatic GST activity was not significantly higher in NBH fish than in SC fish in 1994 (Fig. 2-7A). GST activity remained relatively constant over the first 90 days after removal of fish from the field. There was no consistent sex difference over the same 90 day period. In contrast, in 1997 there was a significant site difference in hepatic GST activity (Fig. 2-8A) 1 day after collection. Hepatic GST rates were approximately twofold higher in NBH fish than in SC fish.

To determine if the site difference observed in the hepatic GST activity of fish collected in 1997 was also present in extrahepatic tissues, GST activity was measured in pools of heart, kidney, gill, and intestine. The extrahepatic tissues examined all had GST activities similar to those found in the liver of fish from the same site, except for the intestines of NBH fish (Fig. 2-8A). GST activity in the intestines of NBH fish was approximately 4 fold higher than the activity in any other tissue and 6.7 fold greater than that in the intestines from SC fish. In general, extrahepatic tissues from NBH fish had higher GST activities than the same tissues from SC fish. These differences were significant for the kidney, gill, and intestine.

\section{UGT:}

In 1994, freshly caught male fish from NBH had much higher UGT activities than any other group of fish (Fig. 2-7B). Over the first 90 days in captivity, UGT activity in each group fluctuated, but some general trends can be identified. UGT activity decreased in NBH males from day 1 to day 8 and then remained relatively constant. In SC and NBH females and SC males over the same period there was no consistent pattern of UGT activities. Hepatic UGT activity was significantly higher in NBH fish collected in ' 97 compared to SC fish collected in the same year, but there was no significant difference between male and female fish (Fig. 2-8B).

Of the extrahepatic tissues, only NBH kidneys and SC hearts had UGT activities similar to those measured in their respective livers. Unlike the GST results, no tissue 
examined had UGT activity higher than that measured in the liver of fish from the same site. As with the GST results, NBH fish had higher UGT activities in all tissues examined (Fig. 2-8B). UGT activity was significantly higher in the kidney, gill, and intestine and slightly higher in the heart $(p=0.08)$. 
Fig. 2-7: Hepatic GST (A) and UGT (B) activity in NBH and SC fish, collected in 1994, $1,8,25$, and 90 days after collection. (A) GST activity in males and females from the same site were not significantly different on any day so combined GST activities are presented. (B) UGT activity significantly differed between sexes from the same site on at least one day. UGT activity in female fish is represented by the first two bar and UGT activity in male fish by the second two bars at each time point. 

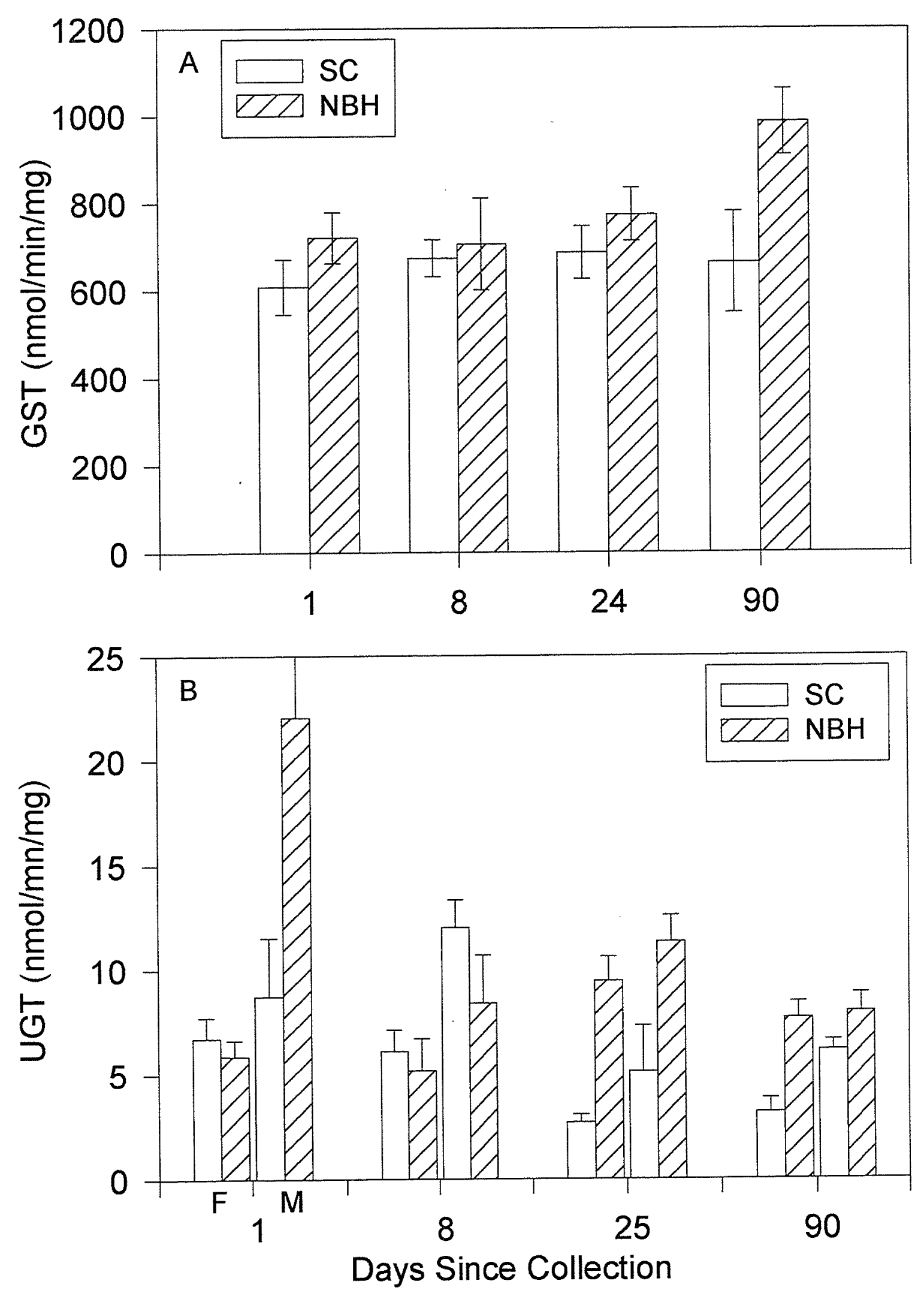
Fig. 2-8: Hepatic and extrahepatic GST (A) and UGT (B) activity in NBH and SC fish collected in 1997. ** NBH activity is significantly different from SC EROD activity ( $\mathrm{p}<$ $0.05,2$-tailed test). (A) GST activity was significantly different in both sex and site ( $\mathrm{p}<$ $0.05,2$-way ANOVA). Only site comparisons were made for extrahepatic tissues due to the low Ns. (B) Combined male and female UGT activities are presented for all tissues. 

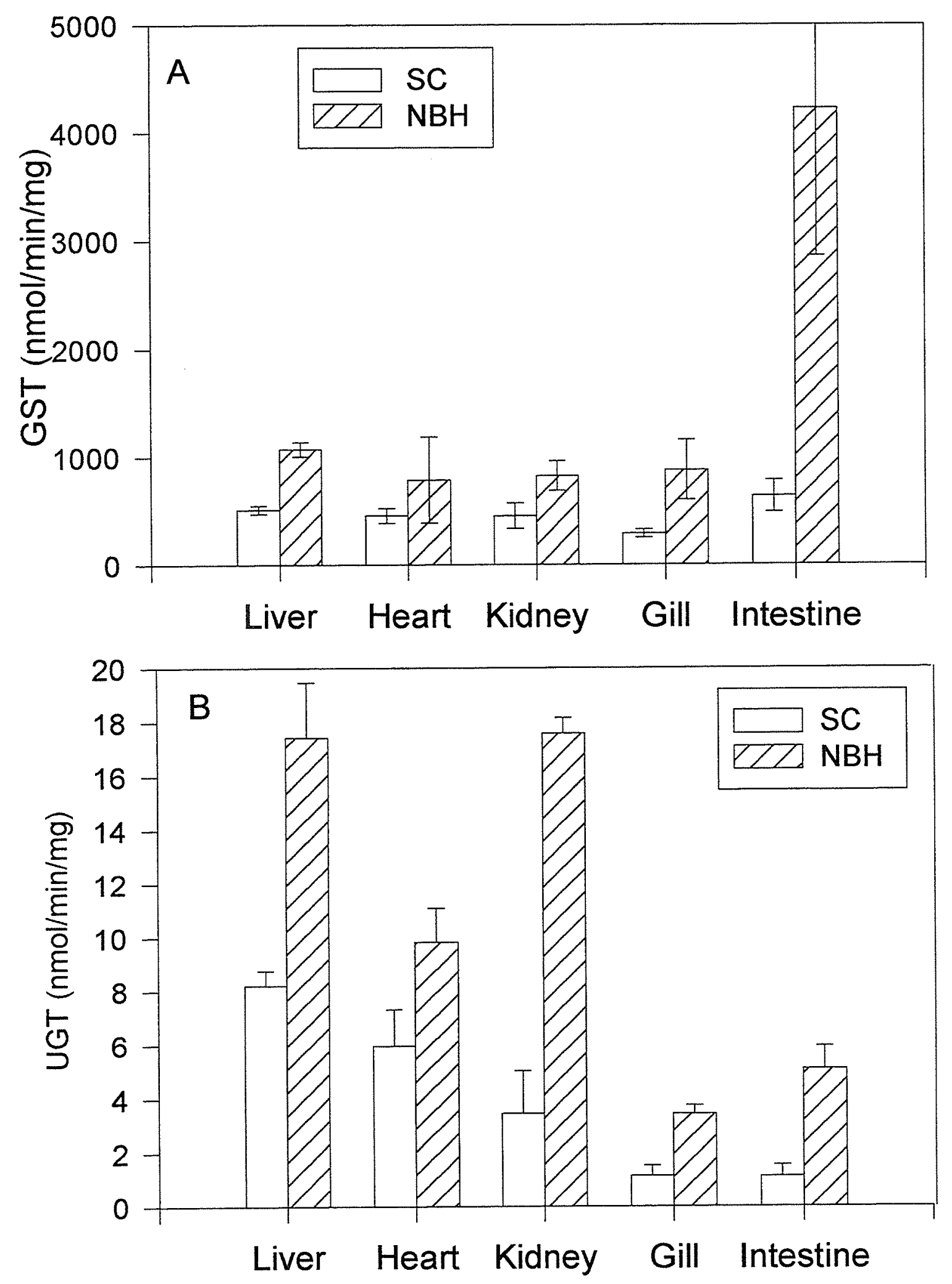


\section{$\underline{\text { Discussion }}$}

Prior studies have found that fish, including Fundulus, inhabiting contaminated sites express elevated levels of CYP1A1 compared to fish from reference sites (Elskus, 1989; Stegeman et al., 1991; Van Veld et al., 1992). Laboratory studies have also demonstrated that CYP1Al in Fundulus can be induced by AhR agonists, including BNF (Kloepper- Sams and Stegeman, 1989), PCBs (Elskus, 1989), and chlorinated dibenzo-pdioxins (Prince, 1995b). Given this background, one would expect that Fundulus inhabiting a site as heavily contaminated as NBH would show elevation of CYP1A1 at some level; activity, protein, and / or mRNA. The results in the present study contradict that expectation.

\section{$\underline{\text { PCB Analysis }}$}

PCB analysis was done in this study primarily to assess the relative contamination of our reference site (SC) compared to our contaminated site (NBH). Results from both the carcass and liver analyses clearly demonstrate that the total PCB content of SC fish is orders of magnitude lower than that of $\mathrm{NBH}$ fish. The comparison of the pattern of $\mathrm{PCB}$ congener distribution in fish from $\mathrm{SC}$ to that in NBH fish yields an interesting difference. In both the carcass and liver, $\mathrm{SC}$ fish have proportionally more of the higher chlorinated PCBs and less of the lower chlorinated PCBs than do NBH fish. This difference could be the result of a number of factors, including the composition of PCBs at the source contamination, the distance of the collection site from the source of the PCBs, the PCB concentration (which can affect the ability of microbes to metabolize PCBs (Yull-Rhee et al., 1993)), and metabolism of the PCBs by the fish. Different AROCLOR mixtures contain relatively more or less chlorine. AROCLORS 1242 and 1016, the major AROCLORS released into NBH (Weaver, 1984), are relatively enriched in the lower chlorinated PCB congeners. Lower chlorinated congeners are more water soluble and may be preferentially lost from the sediment as the distance from the source of contamination 
increases (Pruell et al., 1990). This could result in proportionally higher concentrations of the higher chlorinated congeners in $\mathrm{SC}$, which is presumably farther from its (unknown) source of PCBs. Lower chlorinated PCBs, especially the non-ortho- and mono-orthosubstituted congeners, are preferentially metabolized by CYP1As. This could result in a depletion of these congeners in the organism. A possible example of this is the relative depletion of the lower chlorinated congeners in eels from NBH (Lake, 1995), which have highly induced expression of CYP1Al (Schlezinger and Stegeman, 1999). The relative depletion of the lower chlorinated congeners in livers from $\mathrm{SC}$ fish compared to carcasses of SC fish and not in NBH fish suggests that PCB metabolism in SC fish may partially explain the overall difference in congener distribution between $\mathrm{SC}$ and $\mathrm{NBH}$ fish.

Three other studies have examined the PCB concentrations in NBH Fundulus, either in whole fish (Lake, 1995) (sampled between 1991 and 1993) or in livers (Black et al., 1998) (sampled in 1992 and 1993) (Nacci et al., 1999) (sampled in 1996). In all of these studies Fundulus were collected in the same area of the harbor (the hot spot) from which fish were obtained for this study. The total PCB concentrations reported by Lake etal (1370 ppm) were approximately 5-fold and 1.7-fold higher than the carcass and liver concentrations reported here. This difference may be the result of differences in the number of PCB congeners used to calculate total PCB concentrations, differences in the tissues used in the analysis, and/or actual differences in total PCB concentrations. Substantial dredging has been done in and around the hot spot and the lower PCB concentrations reported here may reflect this decrease in sediment PCB concentrations. When the contribution of individual congeners (on a percent of total PCB weight basis) are compared between the Lake study and this study, the results are remarkably similar. For example congeners 28 and 31 constituted $\sim 14 \%$ of the total in the Lake study and $16 \%$ (in the carcass) in this study, congener 49 constituted $\sim 5.5 \%$ (Lake) and $7 \%$ (this study), and congener 110 (and 77) constituted $\sim 5.5 \%$ (Lake) and $\sim 6 \%$ (this study).

Comparing the $\mathrm{PCB}$ concentrations in from the various liver studies is more problematic. The Black studies only analyzed a few of the mono-ortho and non-ortho 
PCBs. Three of these congeners $(118,105$, and 156) were also measured in this study as part of multi-congener peaks (Table 4); the sum of these three peaks in the Black study (25.8 ug/g) was $\sim 4$-fold lower than in this study $(118.8 \mathrm{ug} / \mathrm{g})$. The Nacci paper reported total PCB concentrations in the livers of NBH Fundulus; PCB concentrations were 324 $\mathrm{ug} / \mathrm{g}$ liver (dry weight). This is $\sim 2.5$-fold lower than the liver concentrations reported in this study. Comparing the same three congeners as above, the data of Nacci etal (38.8 $\mathrm{ug} / \mathrm{g}$, unpublished) is more similar to the results from the Black study than to results in this study. These studies were both conducted at the EPA Naragansette laboratory, suggesting that differences between the data reported here and in these studies is most probably due to differences in methodologies.

The livers from fish from the reference site used in the Black and Nacci studies (West Island) had higher PCB concentrations than SC. Comparing the same three congeners $(118,105$, and 156) as for the NBH fish, the livers from SC fish had PCB concentrations $(0.03 \mathrm{ug} / \mathrm{g})$ an order of magnitude lower than the concentrations found in West Island Fundulus $(0.44 \mathrm{ug} / \mathrm{g})$.

\section{CYP1A1}

Despite their high PCB burdens, NBH Fundulus had consistently low levels of CYP1A1 expression in the liver and several extrahepatic organs. Mean EROD activities in the livers of NBH fish were lower than those in the livers of SC fish. This difference could have been the result of induced expression of CYP1Al in SC fish, but EROD activities in the livers of SC fish were closer to activities seen in control fish compared to Fundulus exposed to BNF (Fig. 2-4). EROD activities in the livers of NBH were consistently equal to or lower than the control EROD activity in the BNF study. EROD activity can be inhibited by high concentrations of specific PCBs (Gooch et al., 1989; Murphy and Gooch, 1997; White et al., 1997); the lower EROD activities in the livers of NBH fish could reflect this. Evidence for inhibition of EROD activity was previously reported for winter flounder sampled from NBH (Elskus et al., 1992; Monosson and Stegeman, 1994). 
Consistent with this, the expression of CYP1A1 protein in the livers of NBH fish was not significantly different from CYP1A1 protein expression in the livers of SC fish. However, both were similar to CYP1A1 protein expression in the livers of control fish from the same TCDF study (0.02 pmol/ug, Ch. 3). Expression of CYP1Al protein can also be suppressed at a post-transcriptional level by concentrations of $\mathrm{PCBs}$ greater than those that suppress EROD activity (Gooch et al., 1989; White et al., 1997). The low CYP1A1 protein expression in the NBH fish could be the result of this type of suppression. However, CYP1Al mRNA expression also was low in the NBH fish. Suppression of CYP1A1 mRNA expression has not been seen in fish at any PCB concentration (up to $10 \mathrm{mg} / \mathrm{kg}$ ) so far tested(Gooch et al., 1989; White et al., 1996), although it is possible that with a sufficiently high concentration of PCBs, CYP1A1 mRNA expression could also be suppressed. Thus the present results suggest that the lack of inducibility of CYP1A1 in NBH fish is either the result of pre-translation suppression of or resistance to induction.

Our observation that EROD activity, CYP1A1 protein and mRNA levels in NBH fish were equivalent to or lower than in fish from a reference site differs from the results of Prince and Cooper (Prince, 1995b), who examined a TCDD resistant population of Fundulus heteroclitus from Newark Bay, NJ. The Newark Bay Fundulus had elevated EROD activity ( 2 to 5 fold) compared to a reference population. Comparing the EROD activities in livers from untreated or control Fundulus from each site showed that the Newark fish (524 \pm 75$)$ had EROD activities that were significantly higher than those of the Tuckerton $(177 \pm 16)$ and NBH $(117 \pm 54)$ and equivalent to those found in SC fish $(390 \pm 85)$. Such differences in the 'resistance phenotype' suggest that each resistant population of Fundulus may have developed a different mechanism(s) for dealing with the toxic chemicals present in their environment. The results of our study provide some insight into the nature of the resistance seen in NBH Fundulus.

The lack of CYP1Al induction in the NBH fish was not limited to the liver, EROD activity, CYP1A1 protein and mRNA levels also were equivalent to those in $\mathrm{SC}$ fish in 
almost all of the extrahepatic tissues examined. In fish from both sites, one cell type in one tissue - the proximal tubules of the kidney - expressed elevated levels of CYP1A1 protein when compared to other cell types in the same fish. The kidney is also the only tissue in which NBH Fundulus had significantly higher CYP1A1 (EROD) activity than SC fish. No significant elevation of CYP1A1 mRNA was detected in kidneys from NBH fish compared to SC fish. This may be the result of the method used to detect the message rather than a lack of induction. The proximal tubules are only $\sim 7 \%$ of the kidney mass and the dot blot technique has an error of $\sim \pm 10 \%$, therefore to detect a difference the CYP1A1 mRNA in the proximal tubules would have to be 20 -fold higher in the NBH fish than in the SC fish.

Elevated levels of CYP1A1 in the kidney proximal tubules have also been shown in control animals in studies using Fundulus larvae (Elskus et al., 1999) and adult fish (Ch. 3; Van Veld et al., 1997) but not in pre-hatch embryos (Toomey, 1999). This would not only suggest that there may be some endogenous upregulation of CYP1A1 in the kidney, but also that a change in the stability, substrate affinity, or metabolic activity of the CYP1A1 protein is not responsible for the low levels of expression of CYP1A1 in other organs of NBH Fundulus.

\section{Phase II Enzymes}

The results from the two phase II enzymes examined suggest that NBH Fundulus are capable of elevated expression of other xenobiotic metabolizing enzymes compared to $\mathrm{SC}$ fish. The consistently significant elevation of both GST and UGT activities in NBH fish from the 1997 collection compared to SC fish from the same collection was not seen in 1994, suggesting that environmental factors other than the presence or absence of contaminants may have been responsible for this difference (see below). The lack of a difference in GST activity in fish collected during the same month (in 1994) differs from results seen in creosote-resistant Fundulus where GST is overexpressed in the liver (and intestine) of resistant fish collected in the same month as fish from a reference site (Van Veld et al., 1991). The tissue specific pattern of GST activity also differs between fish 
from NBH and SC. In NBH fish, GST activity is 4 fold higher in the intestine than in any other tissue; in SC fish GST activity in the intestine is equivalent to the activity in other tissues. Higher levels of GST in the intestine (compared to the liver) of NBH fish is reminiscent of the result with creosote-resistant Fundulus, although the magnitude of the difference is smaller in the creosote-resistant fish ( $\sim 1.3$ fold versus 4 fold in NBH fish) (Van Veld et al., 1991).

Both GST (Hayes and Pulford, 1995) and UGT (Bock, 1991) are multigene families. In mammals, expression of only certain members of these families is controlled by the AhR signal transduction pathway (Sutter, 1992). A few studies in fish have examined the induction of different members of each family following exposure to an AhR agonist (Andersson et al., 1985; Clarke et al., 1992; Leaver et al., 1992; Washburn et al., 1996). Results from the UGT studies (Andersson et al., 1985; Clarke et al., 1992) were similar to those seen in mammalian studies (Bock, 1991) with only the 1-napthol-activity of UGT induced after exposure to AhR agonists. In both the Leaver and Washburn studies, GST activity for CDNB was induced by AhR agonists but the specific GST type responsible for the increase in activity could not be identified.

The assays used in this study to measure GST and UGT activity integrate a number of different members of each enzyme family, some of which may be increasing while others are decreasing in response to any number of environmental stimuli. Some of the differences seen within each data set, as well as between data sets, may be due to differences in the timing of collections. All of the 1994 fish were collected in early to mid July. For this collection, there were no significant inter- or intra-site differences in the condition of the fish, as measured by the LSI and the GSI. In 1997 a two month interval between collection of the SC fish (mid July) and the NBH fish (mid September) resulted in a significant difference in the GSIs of the NBH and SC fish, with the SC fish having a mean GSI approximately 7 fold higher than that of NBH fish. This difference in GSI reflects the fact that the SC fish were gravid at the time of collection while the NBH fish were predominantly gonadally regressed. This may help to explain the significantly higher 
GST and UGT activities in the NBH fish that year, as the expression of members of both families can be altered by steroid hormones (Bock, 1991; Hayes and Pulford, 1995)

\section{Implications for Resistance}

The results of these studies suggest that NBH Fundulus are resistant to CYP1A1 induction by contaminants encountered in their environment, a conclusion supported by results of HAH dosing experiments (see Ch. 3). This resistance extends from EROD activity to the level of mRNA. These results allow us to lay aside some of the many possible explanations for this resistance. The results of assays for UGT and GST activity suggest that the expression of some proteins in NBH fish can be induced, and therefore the resistance to CYP1A1 induction is probably not due to a general loss of inducibility. The general agreement between different measures of CYP1A1 (EROD activity, protein and mRNA expression) in the various tissues examined suggests that inhibition of EROD activity and / or post-translational suppression of CYP1A1 protein are not major factors in the mechanism of resistance. The expression of CYP1A1 in the kidney proximal tubules and the general agreement between different measures of CYP1A1 in the same tissue suggests that the NBH fish do not produce an dysfunctional form of the CYP1A1 protein. Overall these results suggest that there has been an alteration in the function of the AhR signal transduction pathway resulting in increased resistance to the biochemical effects of PCBs. The nature and heritability of this resistance is the subject of continuing research. 
Chapter 3: RESISTANCE TO HALOGENATED AROMATIC HYDROCARBONS IN A CHRONICALLY EXPOSED POPULATION OF FUNDULUS HETEROCLITUS FROM NEW BEDFORD HARBOR: IN VIVO AND IN VITRO STUDIES ON THE INDUCTION OF XENOBIOTIC METABOLIZING ENZYMES IN FERAL FISH.

Introduction $\quad 94$

Materials and Methods $\quad 97$

$\begin{array}{ll}\text { Materials } & 97\end{array}$

Fundulus Collection and Maintenance $\quad 97$

$\begin{array}{ll}\text { Fundulus Treatments } & 97\end{array}$

$\begin{array}{ll}\text { Hepatocyte Culture } & 98\end{array}$

Microsome and Cytosol Preparation 99

Enzyme Activity Assays 99

$\begin{array}{ll}\text { Protein and RNA Blotting } & 101\end{array}$

$\begin{array}{ll}\text { IHC } & 101\end{array}$

$\begin{array}{ll}\text { Results } & 103\end{array}$

$\begin{array}{ll}\text { In Vivo Studies } & 103\end{array}$

Characteristics of SC and NBH Fundulus 103

CYP1A1 Activity and Expression 105

GST Activity 122

UGT Activity 122

In Vitro Studies: CYPIAI Activity 122

$\begin{array}{ll}\text { Discussion } & 127\end{array}$ 


\section{Introduction}

New Bedford Harbor (NBH), MA, is a federal Superfund site contaminated with polychlorinated biphenyls (PCB), polychlorinated-dibenzofurans (PCDF), dibenzo-pdioxins (PCDD), and heavy metals. Sediment PCB concentrations in NBH ranged from a few ppm, in the lower harbor, up to $100,000 \mathrm{ppm}$ in the area closest to the source of PCB contamination (the hot spot) (Weaver, 1984) with a mean in the hotspot of $\sim 350 \mathrm{ppm}$ (Lake, 1995). Fundulus heteroclitus caught in and around the hotspot had body burdens averaging between 260 and $1370 \mathrm{ppm}$ (dry weight) (Ch. 2; Lake, 1995) depending on the time of collection and methods used to determine total PCB concentrations (see Ch. 2).

In the previous chapter we reported that despite their high PCB body burdens Fundulus inhabiting NBH expressed low levels of cytochrome P450 1A1 (CYP1A1) messenger RNA and protein, equivalent to expression in fish from a reference site, Scorton Creek (SC) (Ch 2). These findings suggested that the Fundulus inhabiting NBH may have developed resistance to some or all of the effects of PCBs and other halogenated aromatic hydrocarbons.

The toxic effects of PCBs, as well as other planar halogenated aromatic hydrocarbons ( $\mathrm{pHAH}$ ); including induction of CYP1A1, teratogenicity, thymic atrophy and immunotoxicity, are believed to be dependent on the aryl hydrocarbon receptor (AhR) signal transduction pathway (reviewed in (Landers and Bunce, 1991)). Studies in AhR null mice have conclusively linked the AhR to the nearly all of the toxic effects of TCDD in every organ so far examined (Fernandez Salguero et al., 1996). Fundulus have been shown to have 2 AhRs (Hahn et al., 1994; Hahn et al., 1997), as well as the other components of the AhR signal transduction pathway (Morrison et al., 1998; Powell et al., 1999). Given the predominant role of the AhR pathway in the toxicity of pHAHs in mammals, and the similarity of mammalian and teleost AhR pathways, it is probable that the AhR pathway mediates the toxicity of $\mathrm{pHAHs}$ in fish. Therefore we have focused our studies on the AhR pathway, using CYP1A1 induction as a marker for activation of this pathway. 
Fundulus express an inducible CYP1A1, as has been demonstrated in laboratory induction experiments (Kloepper- Sams and Stegeman, 1989; Gallagher et al., 1995) and through the comparison of fish from relatively contaminated and uncontaminated sites (Burns, 1976; Elskus et al., 1989). A number of recent studies have shown that in Fundulus CYP1Al inducibility can vary depending on prior exposure to inducers (reviewed in (Hahn, 1998)). In these studies CYP1A1 inducibility was impaired in Fundulus exposed to high levels of contaminants (PAHs (Van Veld and Westbrook, 1995), TCDD (Prince, 1995b), PCBs (Elskus et al., 1999; Nacci et al., 1999)) for multiple generations. The same traits that make Fundulus useful as integrators of local contamination - restricted home ranges, non-migratory nature (Lotrich, 1975) and lack of readily dispersed offspring (Brown and Chapman, 1991) - may also increase the probability that a population subject to long-term exposure to high levels of a toxic chemical(s) will develop resistance to that chemical(s).

In this paper we began to investigate the nature of the apparent dioxin resistance of $\mathrm{NBH}$ Fundulus by addressing several questions. Does the resistance to CYP1A1 induction persist in fish that have been held in the laboratory for prolonged periods? Can the resistance be overcome with sufficiently high doses of inducer? Is the resistance limited to halogenated compounds?

To address the first of these questions, we chose to compare the responses of fish from $\mathrm{NBH}$ and $\mathrm{SC}$ after exposure to a potent $\mathrm{AhR}$ agonist, 2,3,7,8tetrachlorodibenzofuran (TCDF). TCDF was chosen as the model inducer because previous experiments in marine teleosts had shown it to be a potent inducer of CYP1A1 (Hahn and Stegeman, 1994). We examined a suite of responses; including induction of CYP1A1 activity, protein, and mRNA, glutathione-S-tranferase (GST) activity, and UDPglucuronosyl transferase activity. These responses were chosen because they are mediated by the AhR signal transduction pathway in mammals (Sutter, 1992).

To address the second and third questions, we chose to examine the inducibility of EROD activity in primary cultures of hepatocytes from NBH and SC fish. The use of 
cultured hepatocytes allowed us to use a wider range of agonist concentrations than was possible using whole fish. The model halogenated agonist for this portion of the study was 2,3,7,8-tetrachlorodibenzo-p-dioxin (TCDD) and the model non-halogenated agonist was $\beta$-naphthoflavone (BNF). TCDD is the most commonly used model agonist for the AhR in mammals (Okey, 1994; Safe and Krishnan, 1995) and is a potent inducer of CYP1A1 in teleosts (Bend et al., 1974; Vodicnik et al., 1981). BNF is also an inducer of CYP1A1 in teleosts (Kloepper- Sams and Stegeman, 1989) and in mammals (Okey, 1994). 


\section{$\underline{\text { Materials and Methods }}$}

Materials: Frozen krill was purchased from MidJersey Pet Supply (Carteret, NJ). APconjugated $2^{\circ} \mathrm{Ab}$ goat anti-mouse was purchased from Bio-Rad (Hercules, CA). Chemiluminescent substrates were purchased from S\&S (Keene, NH) and Tropix (Bedford, MA). UPC-10 Ab was purchased from Organon Teknika (West Chester, PA). A universal immunoperoxidase kit (murine) was purchased from Signet Laboratories (Dedham, MA). ${ }^{32} \mathrm{P}$ was purchased from Dupont NEN (Boston, MA). RNA STAT-60 ${ }^{\mathrm{TM}}$ was purchased from TEL-Test (Friendswood, TX). Permount, SDS and acrylamide were purchased from Fisher (Pittsburgh, PA). All other chemicals were purchased from Sigma (St Louis, MO).

F. heteroclitus collection and maintenance: NBH (6/28 - 6/29/94 \& 7/12-7/14/95) and SC (7/18/94 \& 8/17 - 8/18/95) fish were collected using minnow traps baited with dogfood. Fish were maintained in $20^{\circ} \mathrm{C}$ flowing sea water and fed Tetramin ${ }^{\circledR}$ stapleflake and minced krill.

F. heteroclitus treatment: Fish were moved to 10 gal. experimental aquaria with $20^{\circ} \mathrm{C}$ flowing seawater at least 2 days before the start of the experiment. On the first day of the experiment, fish were weighed and then injected intraperitoneally with $2,3,7,8-$ tetrachlorodibenzofuran (TCDF) dissolved in corn oil, or corn oil alone. Treatment solutions were made so fish received 5 ul per $\mathrm{g}$ of fish. After treatment, fish were held for 7 days in static seawater $\left(\sim 20^{\circ} \mathrm{C}\right)$ during which time they were fed only Tetramin ${ }^{\circledR}$ stapleflake. After 7 days fish were killed by cervical transection. Tissues for microsomes were put immediately in ice cold AhR buffer (see below). Tissues for IHC were collected in biopsy cassettes and placed in 10\% neutral buffered formalin. Tissues for RNA were snap frozen in liquid nitrogen and stored at $-80^{\circ} \mathrm{C}$ until RNA preparation.

Experiment 1: Female fish, which had been held in the laboratory for 9 months, were injected with $0,0.06,0.31,1.5$, or $7.6 \mathrm{nmol} \mathrm{TCDF} / \mathrm{kg}$. There were 4 (control) or 5 
(TCDF) fish per tank, with 1 tank per treatment group per site, for a total of 24 fish per site. Whole livers were collected from individual fish for analysis of CYP1A1 activity and protein, GST activity, and UGT activity. Hearts, gills, and spleens were collected from individual fish for immunohistochemical (IHC) analysis of CYP1A1 protein.

Experiment 2: Male fish, which had been held in the laboratory for 17 months, were injected with 0 or $7.6 \mathrm{nmol}$ TCDF $/ \mathrm{kg}$. (Nine fish from each site were injected at each dose.) After injection, fish from the same site treated with the same dose were randomly assigned to one of three tanks for a total of 3 fish per tank and 3 tanks per dose per site. There was one additional tank, with 3 fish from NBH treated with 50 nmol TCDF $/ \mathrm{kg}$. Individual livers were sub-sampled, into 3 portions, with the major portion $(\sim 2 / 3$ total weight) used for analysis of CYP1A1 activity and protein, GST activity, and UGT activity ( $\mathrm{N}=9$ for all groups except $\mathrm{N}=3$ for $50 \mathrm{nmol} / \mathrm{kg}$ group). Smaller liver portions were used individually for $\mathrm{IHC}$ analysis $\mathrm{N}=9$ for each group except $\mathrm{N}=3$ for $50 \mathrm{nmol} / \mathrm{kg}$ group). Liver potions from three fish (in one tank) were pooled for RNA preparation ( $\mathrm{N}=$ 3 for each group except $\mathrm{N}=1$ for $50 \mathrm{nmol} / \mathrm{kg}$ group). Extrahepatic tissues (heart, gill, intestine, kidney, spleen, and gonad), from one fish in each tank, were preserved for IHC analysis $(\mathrm{N}=3$ for each group except $\mathrm{N}=1$ for $50 \mathrm{nmol} / \mathrm{kg}$ group). The extrahepatic organs from the other two fish in each tank were pooled for RNA preparation $(N=3$ for all groups except $\mathrm{N}=1$ for $50 \mathrm{nmol} / \mathrm{kg}$ group).

Hepatocyte culture: Fish were killed by cervical transection, the livers from 7 (SC) or $5(\mathrm{NBH})$ fish were pooled and placed immediately into $50 \mathrm{ml}$ of ice cold $\mathrm{Ca}^{++}$-free Ringer's solution ( $0.18 \mathrm{M} \mathrm{NaCl}, 1.54 \mathrm{mM} \mathrm{KCl}, 6.49 \mathrm{mM} 7 \mathrm{H}_{2} \mathrm{O}-\mathrm{MgSO}_{4}, 1.45 \mathrm{mM} \mathrm{Na} \mathrm{HPO}_{4}, \mathrm{~K}_{3} \mathrm{PO}_{4}, 0.08 \%$ (w:v) glucose, 2.0\% (w:v) BSA, $0.5 \mathrm{mM}$ EGTA, $10 \mathrm{ml} / 1 \mathrm{P} / \mathrm{S} / \mathrm{A}$ (Sigma, AntibioticAntimycotic solution). Working in a sterile hood, the livers were transferred to a petri dish with $20 \mathrm{ml}$ of fresh $\mathrm{Ca}^{++}$-free Ringer's solution and minced. The minced livers without the Ringer's buffer were transferred to sterile $50 \mathrm{ml}$ centrifuge tubes and incubated for $20 \mathrm{~min}$ in $20 \mathrm{ml}$ of trypsin/EDTA buffer (136 mM NaCl, $11 \mathrm{mM} \mathrm{KCl}, 0.35 \mathrm{mM} \mathrm{KH2PO4,} 0.21 \mathrm{mM} \mathrm{Na} 2 \mathrm{HPO} 4-$ $7 \mathrm{H} 2 \mathrm{O}, 0.24 \mathrm{nM}$ phenol red, $0.003 \%$ penicillin, $0.005 \%$ streptomycin, $0.05 \%$ Trypsin, $0.5 \mathrm{mM}$ 
EDTA). To aid in digestion, the livers were gently homogenized with a Teflon-glass homogenizer. The resulting suspension was filtered through 4 layers of cheesecloth then centrifuged for $5 \mathrm{~min}$ at $100 \mathrm{~g}$ at $4^{\circ} \mathrm{C}$. This pellet was resuspended in $25 \mathrm{ml}$ of MEM plus $10 \%$ calf serum and centrifuged for $5 \mathrm{~min}$ at $100 \mathrm{~g}$. This pellet was resuspended in $10 \mathrm{ml}$ of MEM plus $10 \%$ calf serum and the concentration of cells determined with a hemacytometer. The hepatocytes were plated in 96 well plates at $1 \times 10^{5}$ cells / well and allowed to attach overnight. The hepatocytes were then exposed to TCDD $(0.001-30$ $\mathrm{nM})$ or BNF ( $1-10,000 \mathrm{nM})$ in DMSO or DMSO alone for 24 hours, using methods described previously (Hahn et al., 1996). Four replicate wells were exposed at each concentration.

Microsome and Cytosol preparation: Tissues were homogenized in $9 \mathrm{ml}$ of cold AhR buffer per gram of tissue. AhR buffer consists of $25 \mathrm{mM}$ MOPS (pH 7.5) with $1 \mathrm{mM}$ EDTA, $5 \mathrm{mM}$ EGTA, $0.02 \% \mathrm{NaN}_{3}, 20 \mathrm{mM} \mathrm{Na}_{2} \mathrm{MoO}_{4}, 10 \%$ (v:v) glycerol, $1 \mathrm{mM}$ dithiothreitol, plus protease inhibitors ( $20 \mathrm{uM}$ TLCK, $5 \mu \mathrm{g} / \mathrm{ml}$ leupeptin, $13 \mu \mathrm{g} / \mathrm{ml}$ aprotinin, $7 \mu \mathrm{g} / \mathrm{ml}$ pepstatin A, and $0.1 \mathrm{mM}$ PMFS)(Hahn et al., 1994). Tissues were homogenized with a Teflon-glass homogenizer (10 passes), intestines and gills were minced with dissecting scissors prior to homogenization. Homogenates were centrifuged for $10 \mathrm{~min}$ at $750 \mathrm{~g}$ and $10 \mathrm{~min}$ at $12,000 \mathrm{~g}$ at $4^{\circ} \mathrm{C}$. The supernatant was then centrifuged at $100,000 \mathrm{~g}$ for $70 \mathrm{~min}$ at $4^{\circ} \mathrm{C}$. This supernatant (cytosol) was removed and frozen in liquid $\mathrm{N}_{2}$. The pellet (microsomes) was resuspended in TEDG (0.05M Tris pH 7.5, $1 \mathrm{mM}$ EDTA, $1 \mathrm{mM}$ dithiothreitol, and 20\% glycerol)(Stegeman et al., 1979) and frozen in liquid $\mathrm{N}_{2}$.

EROD activity: CYP1A1 activity in microsomes was determined using a fluorometric, kinetic assay for EROD activity (Hahn et al., 1993). Assays were run in 48 well plates with $2 u \mathrm{M} 7$ ethoxy resorufin and $1.0 \mathrm{mM} \mathrm{NADPH}$ (final concentrations). Samples were scanned once every minute for 15 minutes. A standard resorufin curve (ranging from 0 to 200 pmol of resorufin)was run with each set of samples. EROD activity is presented as pmol resorufin per 
minute per mg total microsomal protein. Samples were run in duplicate. EROD activity in individual wells was linear for at least 10 minutes.

CYP1A1 activity in primary hepatocytes was determined using a fluorometric, stopped assay for EROD activity and total protein ((Kennedy et al., 1995) with modifications by (Hahn et al., 1996)). Briefly medium with inducers was removed and the cells were washed with phosphate-buffered saline (136 mM NaCl, 0.81 mM Na2HPO4, 0.15 mM KH2PO4, $0.27 \mathrm{mM}$ KCL). Phosphate buffer ( $25 \mathrm{mM} \mathrm{Na2HPO4-7H2O} \mathrm{/} \mathrm{NaH2PO4-H2O)} \mathrm{and} \mathrm{7-ER} \mathrm{(} 2 \mathrm{uM}$ ) were added to each sample well and incubated for $10 \mathrm{~min}$ at $\mathrm{rt}$. The reaction was stopped with the addition of cold fluorescamine in acetonitrile $(0.23 \mathrm{pM})$. The plates were then incubated for 10 min at $\mathrm{rt}$ and scanned. BSA and resorufin standards were run on each plate.

Total protein content: Total microsomal or cytosolic protein was measured fluorometrically (Lorenzen and Kennedy, 1993).

GSH transferase (GST) activity: The protocol described in Habig etal (Habig et al., 1974) as modified by Van Veld etal (Van Veld et al., 1991) was followed, with modifications. Briefly, GST activity was measured by adding cytosol ( 0.02 to $0.14 \mathrm{mg}$ total protein) to $1 \mathrm{ml}$ of reaction buffer containing $1 \mathrm{mM}$ 1-chloro-2,4-dinitrobenzene (CDNB), $100 \mathrm{mM}$ Tris-HCl, and $1 \mathrm{mM}$ reduced glutathione and then monitoring the change in absorbance for 1 minute at 344 $\mathrm{nM}$. GST activity was expressed as nmol of conjugate produced per minute per mg total cytosolic protein.

UDP Glucuronosyl Transferase (UGT) activity: The protocol described by Andersson etal (Andersson et al., 1985) was followed, with modifications. Briefly, microsomes (0.01 to 0.11 mg total protein) were added to $0.5 \mathrm{M} \mathrm{KH}_{2} \mathrm{PO}_{4}$ with $p$-nitrophenol $(2.43 \mathrm{mg} / 50 \mathrm{ml})$ and digitonin $(2 \mathrm{mg} / \mathrm{ml})$, plus or minus UDPGA ( $4 \mathrm{mg} / \mathrm{ml})$. This mixture was incubated for $20 \mathrm{~min}$ at RT, shaking in the dark. Then $0.45 \mathrm{ml}$ of $3 \%$ trichloroacetic acid was added and the entire mix centrifuged for $15 \mathrm{~min}$ at $4000 \mathrm{rpm}$. $\mathrm{KOH}(0.05 \mathrm{ml}$ of $5 \mathrm{M})$ was added to $0.375 \mathrm{ml}$ of the supernatant and the absorbance was read at $400 \mathrm{~nm}$. UGT activity was expressed as nmol of conjugate produced per minute per mg total microsomal protein. 
Immunoblotting: CYP1A1 protein content was measured with a chemiluminescent western blot assay (Hahn et al., 1996) using the Scup 1-12-3 monoclonal antibody (MAb) (Park et al., 1986). A standard curve (0.025 to 0.5 pmol CYP1A1) using scup microsomes with known CYP1A1 content was used to determine CYP1A1 protein in the NBH and SC samples. Samples were loaded so that they fell within the range of the standard curve. Samples were run either on $6 \%$ to $15 \%$ acrylamide gradient gels or $10 \%$ acrylamide non-gradient gels. Proteins were transferred to nylon membranes and then incubated with non-specific blocking solution ( $S \& S \otimes$ blocking powder). Membranes were then incubated with MAb 1-12-3 (10 ug/ml) for 1 $\mathrm{hr}$, washed 3 times ( 3 water rinses then a $5 \mathrm{~min}$ wash with $100 \mathrm{ml}$ of tris buffered saline (TBS)) and incubated for $1 \mathrm{hr}$ with $2^{\circ} \mathrm{Ab}$ (AP-conjugated goat anti-mouse, Bio-Rad, 1.1:1000 dilution). Finally the membrane was washed 3 times again, exposed to a chemiluminescent substrate and placed on x-ray film (Kodak, AR). Multiple exposures were taken of each blot. Northern blotting: Total RNA was prepared from frozen tissues using TEL-TEST's RNA STAT $-60^{\mathrm{TM}}$ protocol with modifications. Briefly tissue was homogenized in RNA STAT- $60^{\mathrm{TM}}$ ( $1 \mathrm{ml}$ per 50 to $100 \mathrm{mg}$ of tissue) then incubated at RT for $\sim 5 \mathrm{~min}$. Chloroform was added $(0.2$ $\mathrm{ml}$ per $\mathrm{ml}$ RNA STAT $-60^{\mathrm{TM}}$ ), mixed by inverting and incubated for 2 to $3 \mathrm{~min}$ at $\mathrm{rt}$. This mix was then centrifuged for $15 \mathrm{~min}$ at $4^{\circ} \mathrm{C}$ at $12,000 \mathrm{~g}$ and the aqueous portion transferred to a new centrifuge tube. Isopropanol $\left(0.5 \mathrm{ml}\right.$ per $1 \mathrm{ml}$ RNA STAT- $\left.60^{\mathrm{TM}}\right)$ was added, mixed by inverting, and incubated for 5 to $10 \mathrm{~min}$ at RT. This mixture was centrifuged for $30 \mathrm{~min}$ at $4^{\circ} \mathrm{C}$ at $12,000 \mathrm{~g}$. The supernatant was poured off and the pellet was washed with ice cold $75 \%$ ethanol and air dried. The pellet was dissolved in DEPC-treated water and stored at $-80^{\circ} \mathrm{C}$. RNA (10 ug per lane) was run in 1\% agarose, $3.6 \%$ formaldehyde gels, transferred to nylon membrane overnight and probed with full length ${ }^{32} \mathrm{P}$ labeled $\mathrm{F}$. heteroclitus $\mathrm{CYP} 1 \mathrm{Al}$ [(Morrison et al., 1998).

Immunohistochemistry (IHC): IHC was done according to the methods of Smolowitz et al (Smolowitz et al., 1991), with modifications. In brief, the deparaffinated and hydrated sections were incubated with normal goat serum for 5 minutes to block nonspecific binding of the secondary antibody. Two 1-hour incubations with $150 u \mathrm{~L}$ of monoclonal 
antibody 1-12-3 (1.7 ug/ml in PBS/BSA) were done. The specificity of this antibody for CYP1A has been shown previously (Miller et al., 1989). Sections were washed after this step and the two following steps. The sections were incubated with secondary antibody (Goat antimouse IgG, 1/200 dilution) for 20 minutes, followed by peroxidase-linked mouse IgG, also for 20 minutes. After washing, two 15 minute incubations ( $150 u \mathrm{~L}$ each) of color developer (Signet) were performed. Sections were next counter-stained with Mayers hematoxylin and mounted in crystalmount. Sections of induced and uninduced scup liver were run with each batch of sections as a positive and negative control, respectively. Matching sections were stained with a nonspecific IgG (purified mouse myeloma protein, UPC-10, Organon Teknika, West Chester, PA, $1.7 \mathrm{ug} / \mathrm{ml}$ in PBS/BSA) as a negative control. Sections were read blind and scored on two scales, occurrence: 0 (no cells staining) to 3 (all cells staining) and intensity: 0 (no staining) to 5 (very dark red staining). These 2 scores were multiplied for a final score ("staining index") of 0 to 15 . 


\section{$\underline{\text { Results }}$}

In Vivo Studies:

The low levels of CYP1A1 expression despite high concentrations of PCBs in NBH Fundulus suggested that this population of fish had developed resistance to some AhR agonists (Ch. 2). To test this hypothesis we compared the response of NBH Fundulus and fish from a reference site, $\mathrm{SC}$, to $2,3,7,8-\mathrm{TCDF}$, a potent $\mathrm{AhR}$ agonist.

There was no significant difference in body weight, liver somatic index (LSI), gonad somatic index (GSI), or microsomal yield between control and treated fish from the same site in either experiment 1 (Table 1) or 2 (Table 2). In both experiments, fish from NBH had LSIs that were significantly higher than and GSIs that were significantly lower than those in fish from SC. Mean body weights of fish from NBH were significantly lower than those of fish from SC in experiment 1. In experiment 2 there was no difference in body weights between the sites. 
Table 1: Mean body weight, liver somatic index (LSI), and gonad somatic index (GSI) for experiment 1

\begin{tabular}{|c|c|c|c|c|c|c|c|c|}
\hline & \multicolumn{2}{|c|}{ Body Weight (g) } & \multicolumn{2}{c|}{ LSI (\%) } & \multicolumn{2}{c|}{ GSI (\%) } & \multicolumn{2}{c|}{ Microsomal Yield } \\
\hline $\begin{array}{c}\text { TCDF } \\
(\mathrm{nmol} / \mathrm{kg})\end{array}$ & SC & $\mathrm{NBH}^{*}$ & SC & NBH$^{*}$ & SC & $\mathrm{NBH}^{*}$ & SC & NBH \\
\hline 0 & 11.6 & 6.8 & 5.19 & 6.2 & 18.0 & 8.8 & 9.52 & 11.20 \\
& \pm 0.93 & \pm 0.48 & \pm 0.29 & \pm 0.40 & \pm 0.72 & \pm 2.5 & \pm 2.14 & \pm 2.68 \\
\hline 0.06 & 9.4 & 8.4 & 5.0 & 6.11 & 13.9 & 14.3 & 13.33 & 8.72 \\
& \pm 1.1 & \pm 0.51 & \pm 0.42 & \pm 0.51 & \pm 2.0 & \pm 2.2 & \pm 3.74 & \pm 2.04 \\
\hline 0.31 & 12.0 & 8.0 & 4.14 & 6.0 & 17.7 & 12.7 & 9.86 & 20.72 \\
& \pm 0.95 & \pm 0.58 & \pm 0.16 & \pm 0.42 & \pm 1.8 & \pm 0.84 & \pm 1.49 & \pm 2.11 \\
\hline 1.5 & 11.1 & 7.7 & 4.9 & 6.3 & 18.2 & 13.9 & 13.19 & 9.72 \\
& \pm 0.60 & \pm 0.45 & \pm 0.23 & \pm 0.50 & \pm 2.0 & \pm 1.2 & \pm 3.52 & \pm 2.09 \\
\hline 7.6 & 11.6 & 7.8 & 4.8 & 5.8 & 17.1 & 17.4 & 11.38 & 10.82 \\
& \pm 0.29 & \pm 0.66 & \pm 0.36 & \pm 0.31 & \pm 0.80 & \pm 4.3 & \pm 0.70 & \pm 0.56 \\
\hline
\end{tabular}

The LSI is the ratio of the total weight of the liver compared to the total weight of the fish. The GSI is the ratio of the total weight of the gonad compared to the total weight of the fish. *The sites were significantly different $(\mathrm{p}<0.05)$ in a 2 way ANOVA comparing site and dose

Table 2: Mean body weight, liver somatic index (LSI), and gonad somatic index (GSI) for experiment 2

\begin{tabular}{|c|c|c|c|c|c|c|c|c|}
\hline & \multicolumn{2}{|c|}{ Body Weight (g) } & \multicolumn{2}{c|}{ LSI (\%) } & \multicolumn{2}{c|}{ GSI (\%) } & \multicolumn{2}{c|}{ Microsomal Yield } \\
\hline $\begin{array}{c}\text { TCDF } \\
(\mathrm{nmol} / \mathrm{kg})\end{array}$ & SC & NBH & SC & NBH $^{*}$ & SC & NBH* $^{*}$ & SC & NBH \\
\hline 0 & 7.7 & 6.7 & 2.2 & 3.3 & 3.0 & 1.7 & 4.61 & 6.77 \\
& \pm 0.41 & \pm 0.53 & \pm 0.22 & \pm 0.16 & \pm 0.26 & \pm 0.25 & \pm 0.72 & \pm 0.66 \\
\hline 7.6 & 6.8 & 7.1 & 2.8 & 3.2 & 3.76 & 1.3 & 6.73 & 8.58 \\
& \pm 0.44 & \pm 0.53 & \pm 0.23 & \pm 0.32 & \pm 0.29 & \pm 0.25 & \pm 0.6 & \pm 0.86 \\
\hline 50 & + & 7.9 & & 3.0 & & 0.83 & & 6.48 \\
& & \pm 1.4 & & \pm 0.72 & & \pm 0.25 & & \pm 0.83 \\
\hline
\end{tabular}

The LSI is the ratio of the total weight of the liver compared to the total weight of the fish. The GSI is the ratio of the total weight of the gonad compared to the total weight of the fish. *The sites were significantly different $(\mathrm{p}<0.05)$ in a 2 way ANOVA comparing site and dose. No SC fish were treated with $50 \mathrm{nmol} \mathrm{TCDF} / \mathrm{kg}$. 
CYP1A1: In experiment 1, fish from SC responded to increasing doses of TCDF with increased CYP1Al expression. In the liver this increase in CYP1A1 could be measured as increased EROD activity and CYP1A1 protein (Fig. 3-1A \& B). Using IHC analysis induction of CYP1A1 protein could also be seen in the vascular endothelium of the gonad, spleen, and gill as well as in the heart (Fig. 3-2A \& B and Fig. 3-3). In contrast, no induction could be seen in the fish from NBH, by any measure, in any tissue (Fig. 3-1A \& B; Fig. 3-2 C \& D; Fig. 3-3).

In experiment 2, fish from both sites had induced EROD activity, although the dose required to achieve induction and the magnitude of the inducted CYP1A1 expression differed. In SC fish the $7.6 \mathrm{nmol} \mathrm{TCDF} / \mathrm{kg}$ dose significantly induced EROD activity as compared to controls $(p<0.01)$ (Table 3). EROD activity in the livers of fish from NBH was not significantly induced by $7.6 \mathrm{nmol} \mathrm{TCDF} / \mathrm{kg}$, but it was significantly induced by $50 \mathrm{nmol}$ TCDF $/ \mathrm{kg}(\mathrm{p}<0.001)$. The EROD activity induced in fish from NBH by this dose was only $900 \mathrm{pmol} / \mathrm{min} / \mathrm{mg}$; compared to $2850 \mathrm{pmol} / \mathrm{min} / \mathrm{mg}$ in fish from SC by treated with $7.6 \mathrm{nmol} / \mathrm{kg}$. Different results were found when CYP1A1 protein (Table 3) was measured. Fish from SC had significantly elevated levels of CYP1A1 $(p<0.005)$. However, fish from NBH also had significantly higher levels of CYP1A1 in the $7.6 \mathrm{nmol} /$ $\mathrm{kg}$ group as well as in the $50 \mathrm{nmol} / \mathrm{kg}$ group $(\mathrm{p}<0.05)$. Again, the magnitude of the induced expression differed between fish from the two sites. CYP1A1 levels induced by either dose in fish from NBH was $0.02 \mathrm{pmol} / \mathrm{ug}$, equal to the expression in control fish from SC, and much lower than that induced in SC fish $(0.08 \mathrm{pmol} / \mathrm{ug})$. Unlike both the EROD activity and CYP1A1 protein results, CYP1Al messenger RNA content was not induced in fish from $\mathrm{NBH}$ at any dose. CYP1Al mRNA was induced in the fish from $\mathrm{SC}$ (Fig. 3-4). 
Fig. 3-1: Hepatic induction of CYP1Al (EROD) activity (A) and protein (B) in SC (open bars) and NBH (hatched bars) Fundulus injected with corn oil, or $0.06,0.31,1.5$, or 7.6 nmol TCDF / kg dissolved in corn oil. Each bar is the mean $( \pm \mathrm{SE})$ of 4 (corn oil) or 5 (TCDF) fish. * Significantly different from the reference site (marked in the legend) or from the control from the same site ( $p<0.01,2$-way ANOVA comparing site and dose) 

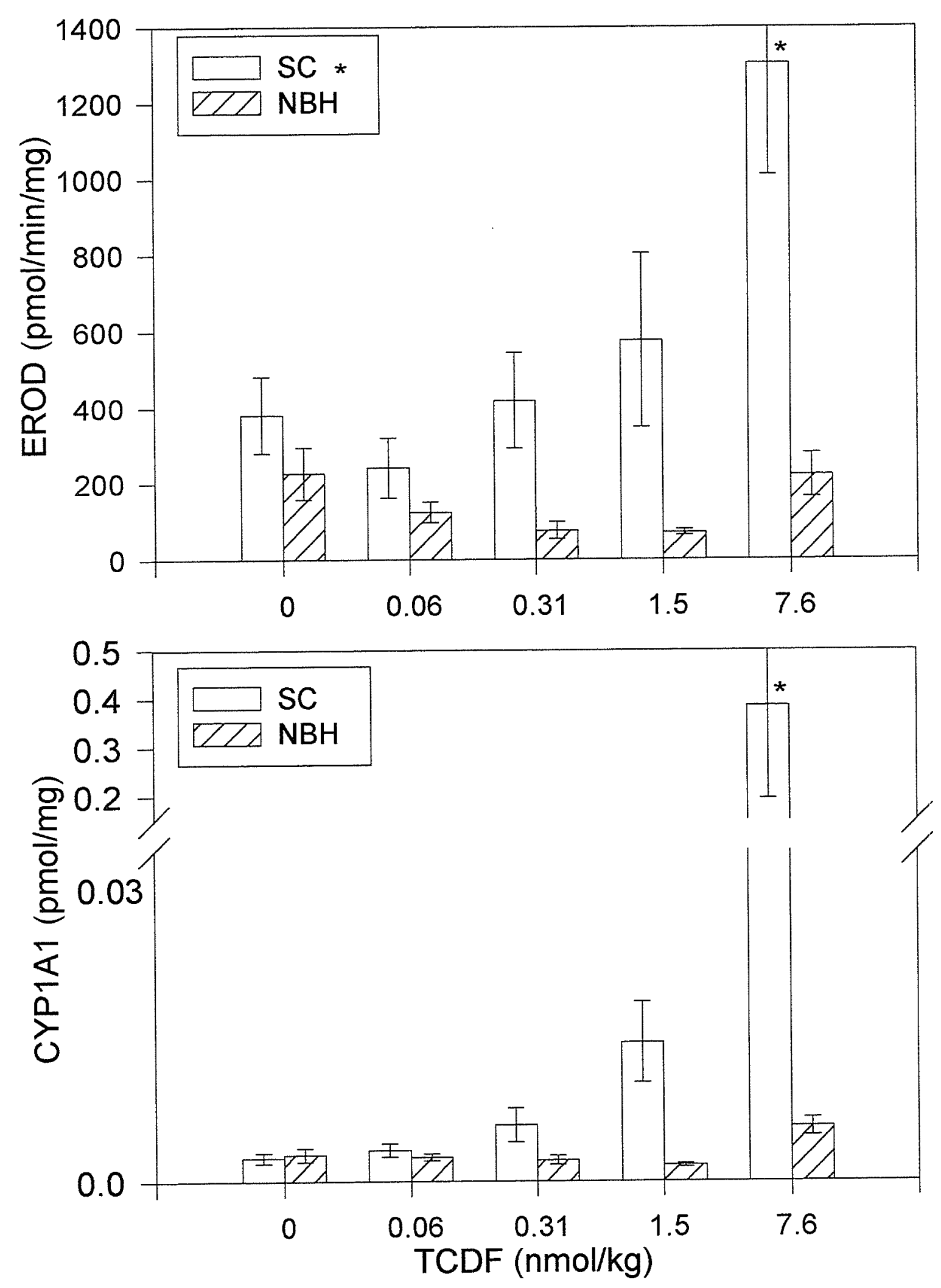
Fig. 3-2: CYP1Al expression in the atria of $\mathrm{SC}$ and $\mathrm{NBH}$ Fundulus injected with corn oil or $7.6 \mathrm{nmol}$ TCDF / $\mathrm{kg}$ dissolved in corn oil. (A) SC corn oil (B) SC TCDF (C) NBH corn oil (D) NBH TCDF 

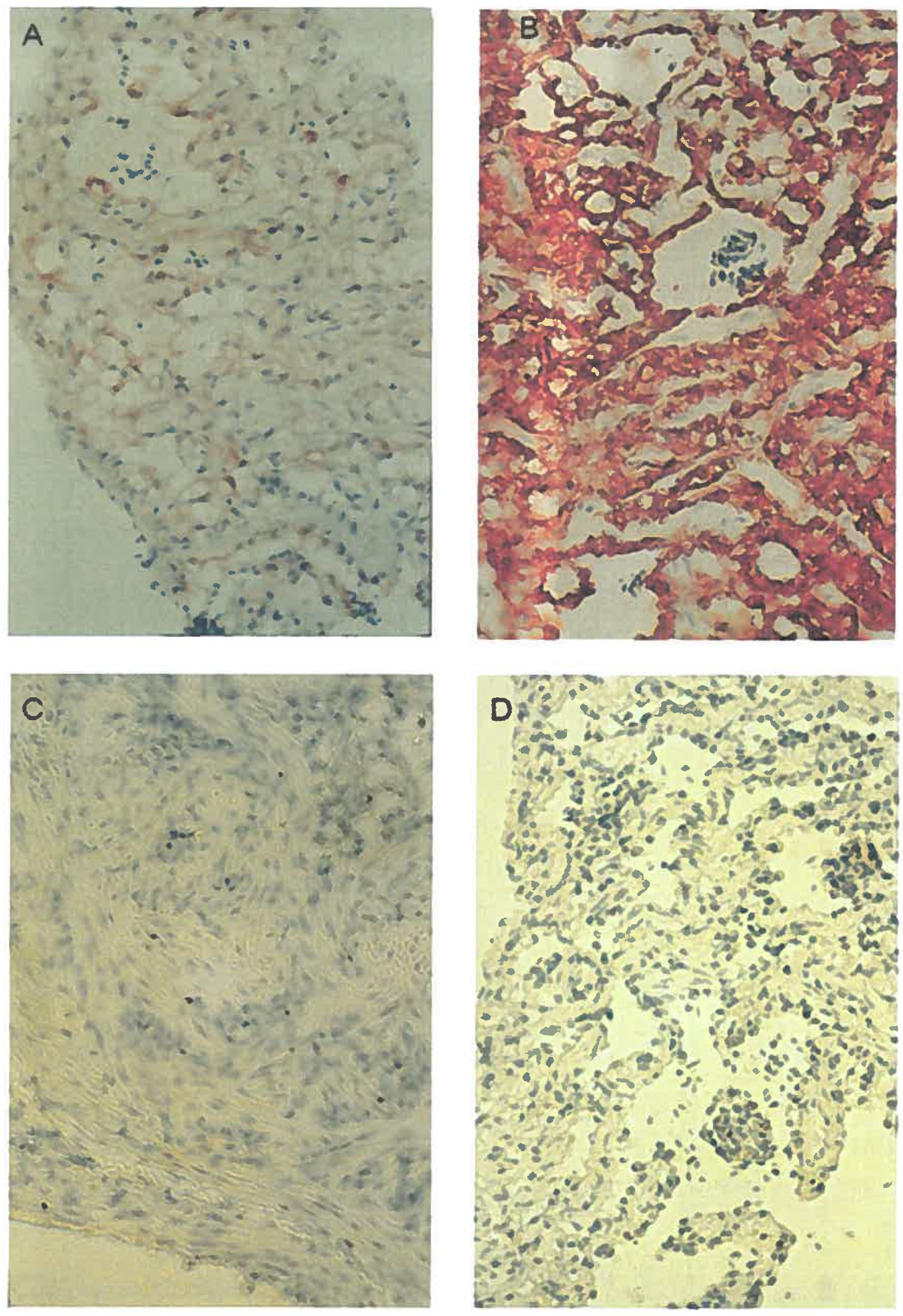
Fig. 3-3: CYP1A1 expression in the vascular endothelium of the (A) atrium and ventricle or (B) spleen and gonad of SC and NBH Fundulus injected with corn oil, or $0.31,1.5$, or $7.6 \mathrm{nmol}$ TCDF / $\mathrm{kg}$ dissolved in corn oil. *Significantly different ( $\mathrm{p}<0.01$, 2-way ANOVA comparing site and dose) from control fish from the same site. Overall mean NBH IHC scores for each tissue were significantly different ( $p<0.01,2$-way ANOVA) from the mean SC IHC score for the same tissue. 

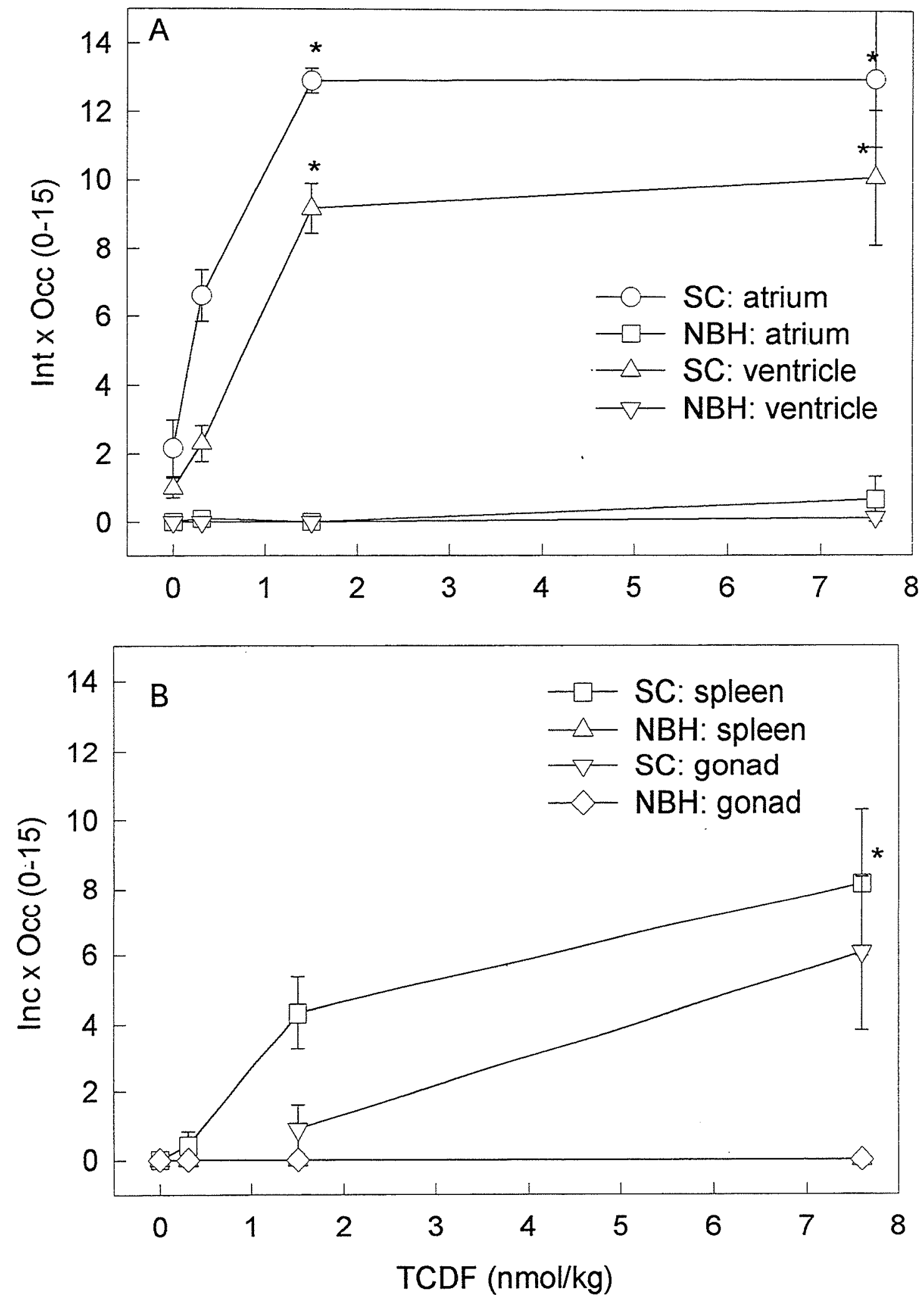
Fig. 3-4: Northern blots of total RNA from the liver (A), heart (B), kidney (C) and gill (D) of SC and NBH Fundulus injected with corn oil or 7.6 or $50 \mathrm{nmol} \mathrm{TCDF} / \mathrm{kg}$. Total RNA (10 ug per lane) from 3 (liver) or 2 (extrahepatics) fish was pooled and 3 pools per treatment ( 1 pool for $50 \mathrm{nmol} \mathrm{TCDF} / \mathrm{kg}$ ) were used for Northern blots with hybridization to Fundulus CYP1A1 cDNA probe as described in materials and methods. Individual tissues from the same 2 or 3 fish were pooled for each of the tissues examined and were loaded in the same position in each gel. Lanes 1-3: NBH corn oil; lanes 4-6: SC corn oil; lanes 7-9: NBH TCDF (7.6 nmol/kg); lanes 10-12: SC TCDF (7.6 nmol/kg); lane 13: $\mathrm{NBH}$ TCDF $(50 \mathrm{nmol} / \mathrm{kg})$. 


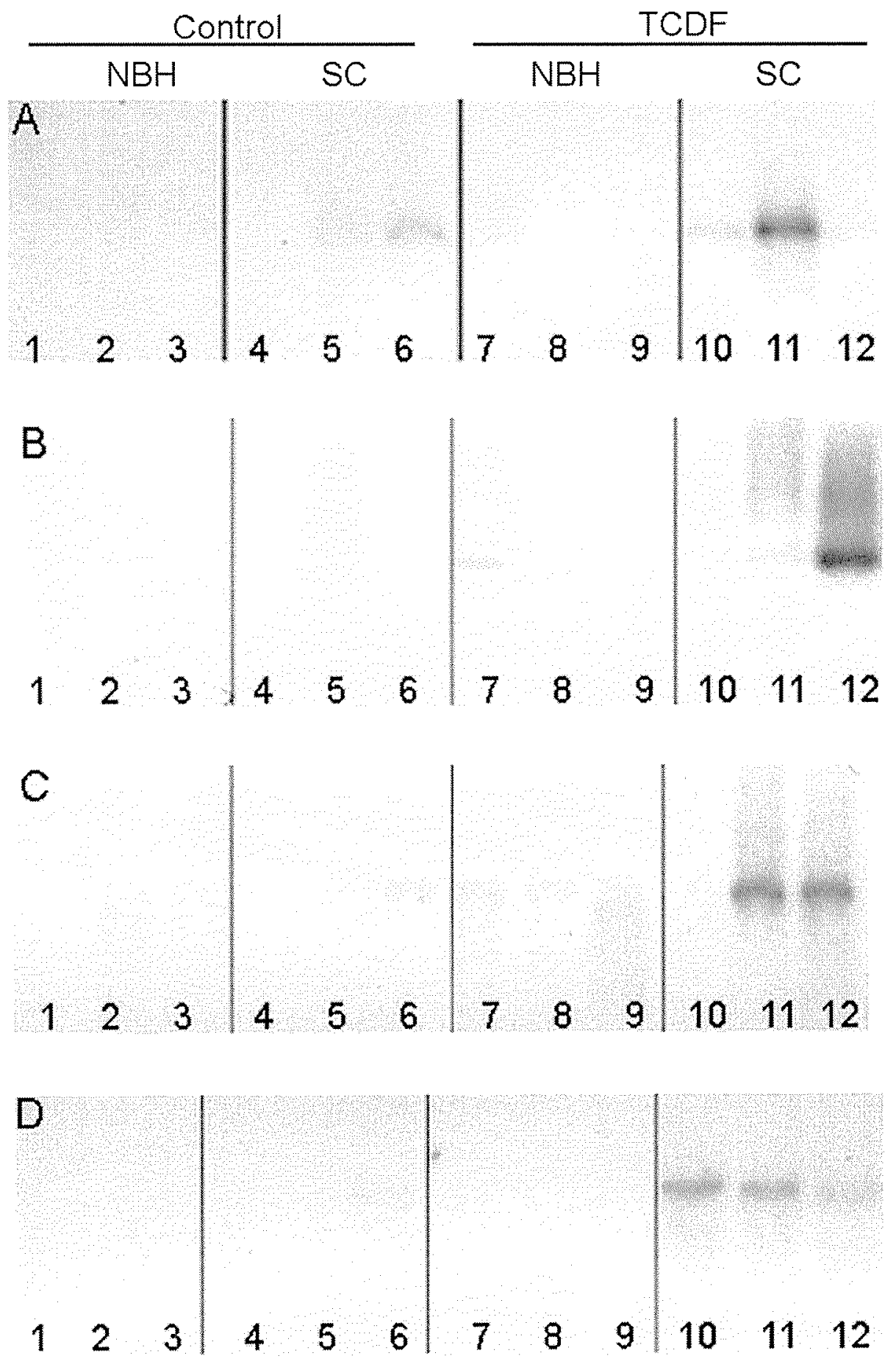


Fig. 3-5: IHC analysis of CYP1A1 expression in extrahepatic tissues (heart, kidney, intestine, and gill) of $\mathrm{SC}$ and $\mathrm{NBH}$ Fundulus injected with corn oil or $7.6 \mathrm{nmol} \mathrm{TCDF} / \mathrm{kg}$. Tissues are scored on two scales: Occurrence (Occ, 0-3) and Intensity (Int, 0-5) the results of these two measurements are multiplied to give a final (Occ $\mathrm{x}$ Int) score from 0 to 15. Open bars: SC corn oil; forward-slashed bars: SC TCDF; back-slashed bars: NBH corn oil; cross hatched bars: NBH TCDF. UD: no CYP1A1 protein was detected (0 Occ $\mathrm{x}$ Int). *Significantly different from the reference site (marked next to the $\mathrm{X}$ axis label) or from the control from the same site $(p<0.01,2$-way ANOVA comparing site and dose) 


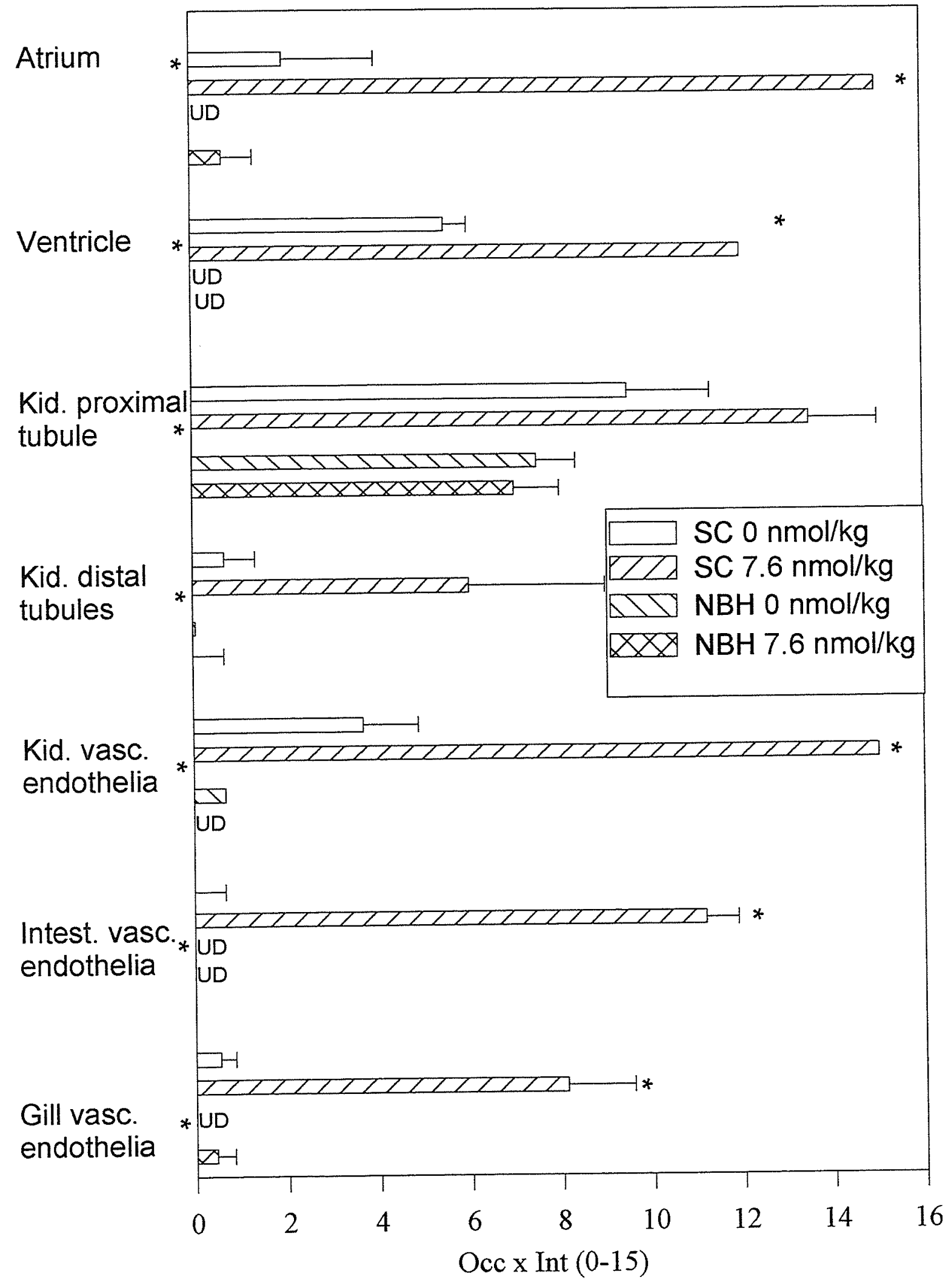


IHC analysis of CYP1A1 expression in extrahepatic tissues revealed differential inducibility like that in the liver. In the SC animals CYP1A1 protein was induced in the vascular endothelium of the gill, intestine, gonad and kidney as well as in the heart ( $\mathrm{p}<$ 0.001) (Fig. 3-5). There was no significant induction in any extrahepatic non-endothelial cell types. However, there was a significant difference in overall CYP1A1 expression between the sites in the kidney proximal and distal tubules $(p<0.05)$. Interestingly, as in fish collected from the field (Ch. 2), fish from NBH and SC had moderate levels of CYP1Al expression in the proximal tubules regardless of treatment. This was the only extrahepatic tissue in which fish from NBH consistently expressed CYP1A1. CYP1A1 protein expression was not induced in any tissue in the fish from NBH.

Analysis of CYP1A1 mRNA expression in extrahepatic tissues confirmed these results (Fig. 3-4). CYP1A1 mRNA was induced in the heart, kidney, gill, and intestine of $\mathrm{SC}$ fish. CYP1Al mRNA was not induced in any tissue in fish from $\mathrm{NBH}$, nor in the spleen or gonad of fish from SC. In the induced fish from SC the magnitude of induction varied greatly between pools of RNA from the same treatment group. The magnitude of induction in tissues pooled from the same pair of fish also varied. 
Table 3: CYPIAl activity and protein in SC and NBH Fundulus treated with 2,3,7,8TCDF

\begin{tabular}{|l|l|l|l|l|}
\hline & \multicolumn{2}{|c|}{$\begin{array}{c}\text { CYP1A1 (EROD) Activity } \\
\text { (pmol/min/mg) }\end{array}$} & \multicolumn{2}{c|}{ CYP1A1 Protein (pmol/ug) } \\
\hline Site & \multicolumn{1}{|c|}{ SC } & \multicolumn{1}{c|}{$\mathrm{NBH}^{*}$} & SC & $\mathrm{NBH}^{*}$ \\
\hline $0 \mathrm{nmol} \mathrm{TCDF} / \mathrm{kg}$ & $736 \pm 139$ & $289 \pm 51$ & $0.02 \pm 0.005$ & $0.007 \pm 0.002$ \\
\hline $\begin{array}{l}7.6 \mathrm{nmol} \mathrm{TCDF} / \\
\mathrm{kg}\end{array}$ & $2850 \pm 530^{*}$ & $424 \pm 67$ & $0.08 \pm 0.02^{*}$ & $0.02 \pm 0.004^{*}$ \\
\hline $\begin{array}{l}50 \mathrm{nmol} \mathrm{TCDF} / \\
\mathrm{kg}\end{array}$ & ND & $901 \pm 283^{*}$ & $\mathrm{ND}$ & $0.02 \pm 0.006^{*}$ \\
\hline
\end{tabular}

$\mathrm{ND}$ : not determined 
Fig. 3-6: GST activity in SC (open bars) and NBH (hatched bars) Fundulus from experiments 1 (A) and 2 (B). (A) Fundulus were treated with corn oil or $0.06,0.31,1.5$, or $7.6 \mathrm{nmol} \mathrm{TCDF} / \mathrm{kg}$ dissolved in corn oil. Bars are the mean $( \pm \mathrm{SE})$ of 4 (corn oil) or 5 (TCDF) fish. There was no significant difference in GST activity between TCDF doses or sites (2-way ANOVA comparing site and dose). (B) Fundulus were treated with corn oil or 7.6 or 50 (NBH only) nmol TCDF / kg dissolved in corn oil. Bars are the mean $( \pm \mathrm{SE})$ of 9 fish except for the $50 \mathrm{nmol} \mathrm{TCDF} / \mathrm{kg}$ which is the mean $( \pm \mathrm{SE})$ of 3 fish. There was no significant difference in GST activity between doses in SC fish. There was a significant difference in GST activity between doses in NBH fish ( $p<0.005$, 2-way ANOVA comparing site and dose) 

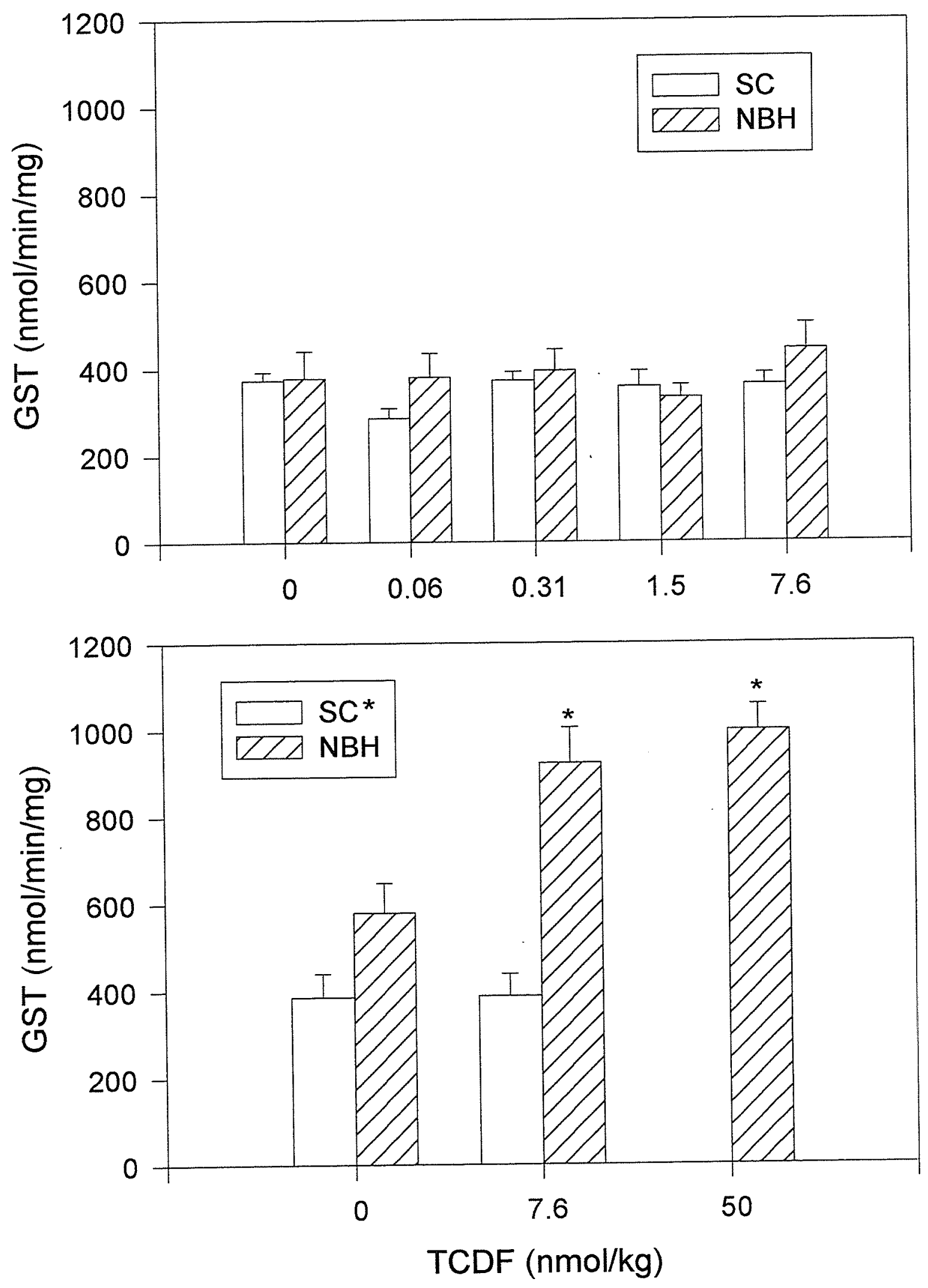
Fig. 3-7: UGT activity in SC (open bars) and NBH (hatched bars) Fundulus from experiments 1 (A) and 2 (B). (A) Fundulus were treated with corn oil or $0.06,0.31,1.5$, or $7.6 \mathrm{nmol} \mathrm{TCDF} / \mathrm{kg}$ dissolved in corn oil. Bars are the mean $( \pm \mathrm{SE}$ ) of 4 (corn oil) or 5 (TCDF) fish. There was no consistent trend in UGT activity compared to TCDF dose. There was no significant difference between sites (2-way ANOVA comparing site and dose). (B) Fundulus were treated with corn oil or 7.6 or 50 (NBH only) nmol TCDF / kg dissolved in corn oil. Bars are the mean $( \pm \mathrm{SE}$ ) of 9 fish except for the $50 \mathrm{nmol}$ TCDF $/ \mathrm{kg}$ which is the mean $( \pm$ SE) of 3 fish. There was no significant difference in UGT activity between doses in SC or NBH fish. There was a significant difference in UGT activity between sites when only the corn oil and $7.6 \mathrm{nmol} \mathrm{TCDF} / \mathrm{kg}$ treatment groups were compared ( $\mathrm{p}<0.005,2$-way ANOVA comparing site and dose). 

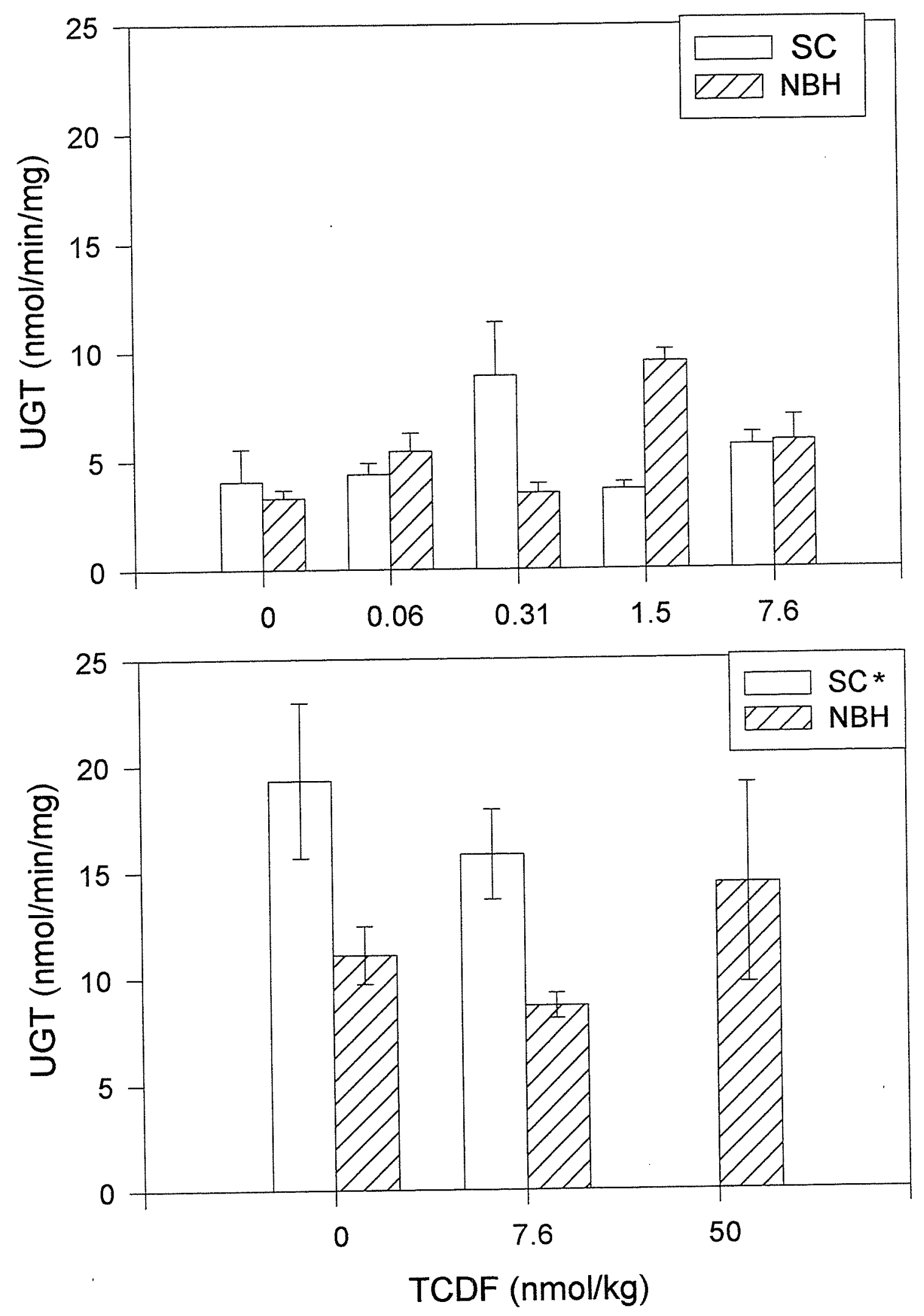
GST: Rates of GST activity with CDNB in NBH and in SC females (experiment 1) did not change in response to TCDF treatment (Fig. 3-7A). Similarly, in experiment 2 there was no change in rates of GST activity with TCDF treatment in the SC (male) fish. In the NBH male fish, however, there was a significant increase in GST activity with TCDF treatment $(p<0.005)$ (Fig. 3-7B).

UGT: In experiment 1, UGT activity was variable in fish from both sites, but lacked any consistent trend related to TCDF dose (Fig. 3-8A). There was no significant difference in UGT activity between sites in this experiment. In experiment 2 , as in experiment 1 , there was no significant difference in UGT activity with TCDF treatment (Fig. 3-8B). But there was a significant difference between sites, fish from NBH having lower UGT activities than fish from SC $(\mathrm{p}<0.005)$; UGT activity in SC male fish was $1.7-1.8$ fold higher than the activity in NBH male fish.

\section{In Vitro Studies:}

The in vivo dosing studies described above did not reveal the magnitude of the difference in sensitivity to HAH between the NBH and SC Fundulus, nor did they allows us to ascertain whether the fish from NBH were capable of more than a minimal response. To address these questions, CYP1A1 inducibility was assessed in primary hepatocytes treated with TCDD. Primary hepatocyte cultures from both NBH and SC fish responded to TCDD treatment with increased EROD activities (Fig. 3-9A). There was no difference in the maximal EROD activity reached after TCDD treatment of hepatocytes from the two sites. However, the TCDD dose response curve for hepatocytes from $\mathrm{NBH}$ fish was shifted to the right, resulting in an EC50 for TCDD that was 14-fold higher than the EC50 for TCDD in hepatocytes from fish from SC (Table 4).

To determine if the decreased sensitivity of NBH Fundulus to TCDD and TCDF is reflected in decreased sensitivity to a non-halogenated AhR agonist, primary hepatocytes from $\mathrm{SC}$ and $\mathrm{NBH}$ fish were treated with $\mathrm{BNF}$. As with TCDD, there was no difference 
between sites in the maximal EROD activity (Fig. 3-9B, Table 4). However, the EC50 for CYP1Al induction in hepatocytes from fish from NBH was only 3-fold greater than the EC50 for CYP1A1 induction in hepatocytes from fish from SC (Table 4). 
Fig. 3-8: CYP1A1 (EROD) activity in primary cultures of hepatocytes, from SC or NBH Fundulus, treated with TCDD (A) or BNF (B). Results from duplicate plates are shown, each curve is the mean of 3 replicate wells. (A) Hepatocytes were treated with DMSO or 2,3,7,8TCDD $(0.001-30 \mathrm{nM})$ and EROD activity was measured 24 hours later. Hepatocytes from NBH fish (EC50: 0.604nM) were 14-fold less sensitive to TCDD than hepatocytes from SC fish (EC50: 0.043nM). (B) Hepatocytes were treated with DMSO or BNF (1 - 10,000 nM) and EROD activity was measured 24 hours later. Hepatocytes from NBH fish (EC50:350nM) were only 3-fold less sensitive to BNF than hepatocytes from SC fish (EC50: 113nM). Maximal EROD activity was similar booth between sites and between compounds. (This experiment was performed by Diana Franks) 


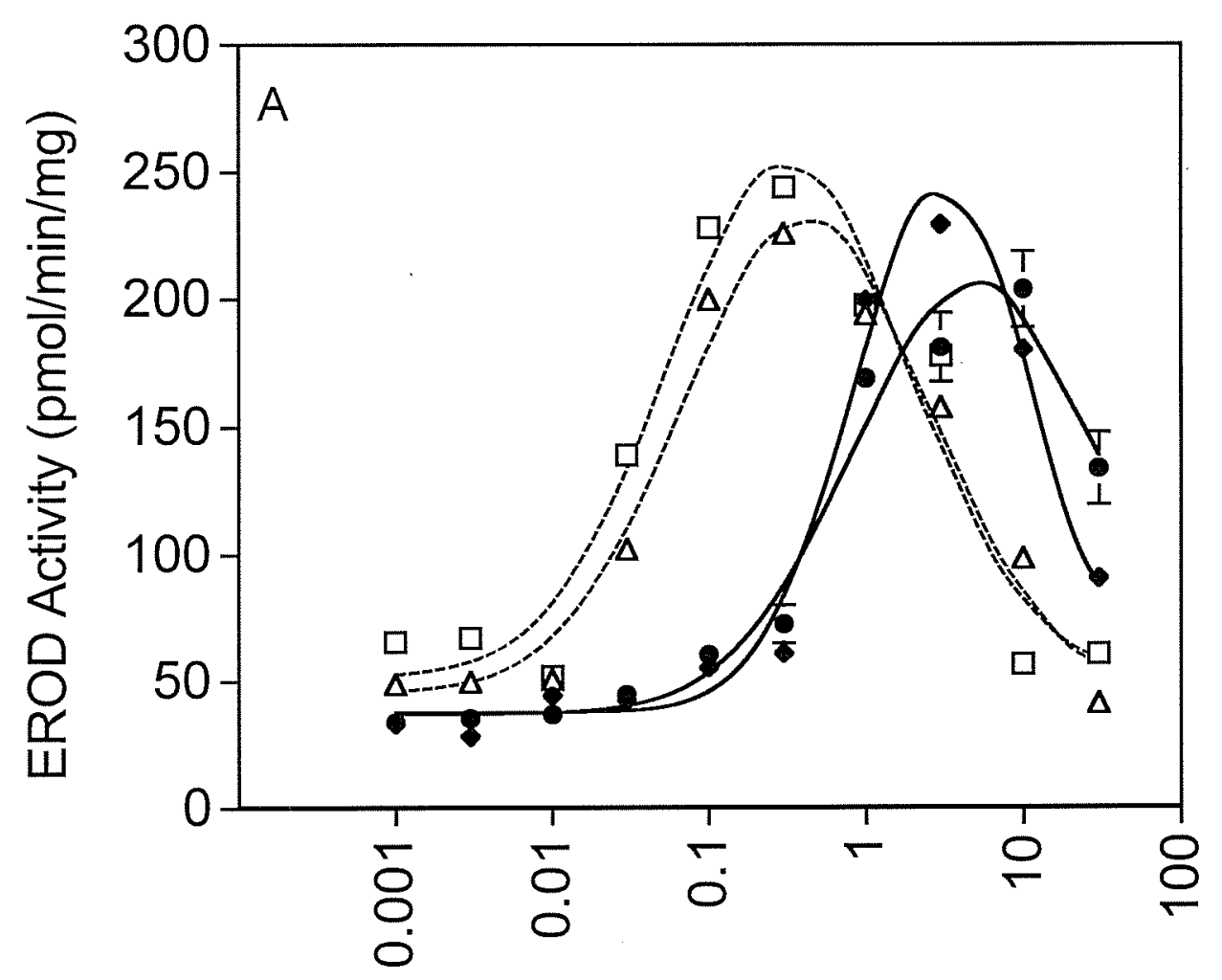

[TCDD] (nM)

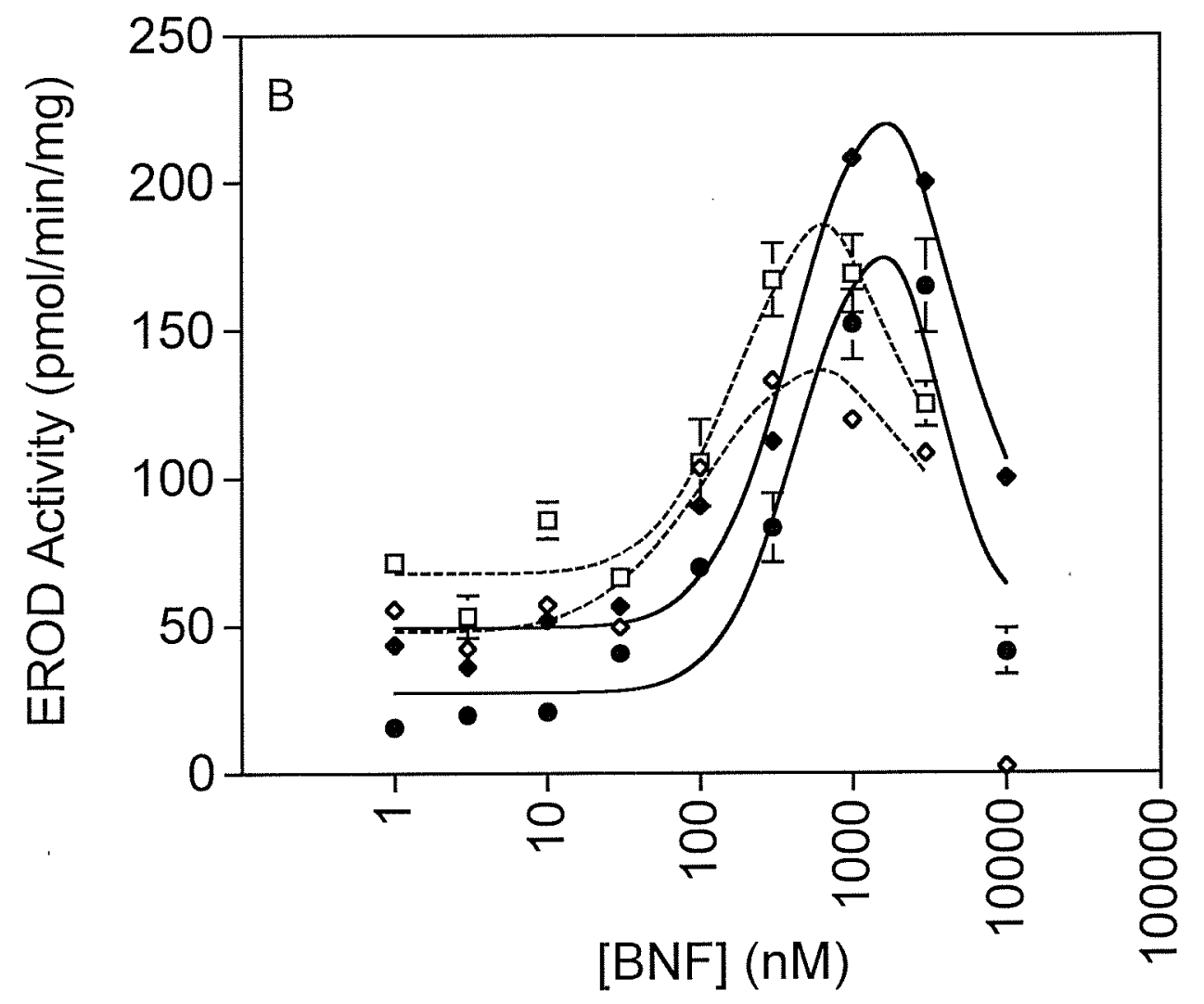


Table 4: Sensitivity of primary cultures of Fundulus hepatocytes to CYP1A1 induction by TCDD and BNF

\begin{tabular}{|l|l|l|l|l|l|}
\hline & \multicolumn{2}{|c|}{$\begin{array}{c}\text { EROD EC50 } \\
(\mathrm{nM})\end{array}$} & Ratio of EC50s & \multicolumn{1}{|c|}{$\begin{array}{c}\text { Maximum EROD response } \\
\text { (pmol/min/mg) }\end{array}$} \\
\hline Inducer & SC & NBH & (NBH/ SC) & SC & NBH \\
\hline TCDD & $0.045,0.040$ & $0.582,0.625$ & $\sim 14$ & 226,244 & 204,229 \\
\hline BNF & 85,141 & 360,339 & $\sim 3$ & 133,169 & 165,208 \\
\hline Comparison to other fish cells & \multicolumn{3}{l|}{} \\
\hline Inducer & Cells (species) & EC50 & 0.025 \\
\hline TCDD & RTG-2 (trout) & $0.13,0.21$ \\
\hline TCDD & PLHC-1 (Poeciliopsis) & \\
\hline TCDD & (Zebrafish) $^{3}$ & 0.54 \\
\hline
\end{tabular}

EC50 is the concentration of inducer required to achieve half maximal induction. Values presented are the individual means of duplicate plates; each mean was is determined from 3 replicate wells. ${ }^{1}$ CYP1A1 mRNA; Zabel, $1996{ }^{2}$ EROD and CYP1A1 protein; Hahn, $1996{ }^{3}$ CYP1A1 mRNA; Henry, 1996 


\section{Discussion}

\section{In Vivo Studies}

In previous studies, we have shown that Fundulus from NBH appear to be resistant to CYP1A1 induction by $\mathrm{AhR}$ agonists encountered in their environment. In the present studies, we examined the ability of the fish from NBH to respond to laboratory treatment with model AhR agonists. The results of treatments with TCDF demonstrate that fish from $\mathrm{NBH}$ do not recover normal CYP1A1 inducibility even after prolonged periods ( $9-17$ months) in clean water. As in the field studies, the lack of inducibility of CYP1Al in NBH fish was not limited to the liver, but was found in every tissue examined.

In this as in the previous study (Ch. 2), the proximal tubules of the kidneys continue to be the one cell type in which fish from NBH expressed readily detectable levels of CYP1A1. Control and TCDF-treated animals from both sites were found to express similar moderate levels of CYP1A1 in these cells. This pattern of expression has also been detected by other groups, in adult fish (Van Veld et al., 1997) and in larvae (Cantrell et al., 1998; Elskus et al., 1999).

We also examined the response of two phase II enzymes, GST and UGT. Some members of the GST and UGT gene families are part of the mammalian AhR gene battery (Nebert et al., 1993). In experiment 1, where female fish were exposed to multiple TCDF doses $(0.06-7.6 \mathrm{nmol} / \mathrm{kg})$, neither GST nor UGT differed significantly in relation to TCDF dose and the lack of response of animals from both sites was similar. In experiment 2, where male fish were exposed to TCDF ( 7.6 or $50 \mathrm{nmol} / \mathrm{kg}$ ), a dose dependent increase in GST occurred only in the fish from NBH, while UGT activity differed between sites but was not inducible. Such conflicting results are common when using these assays. Unlike the assay used to measure EROD activity, the measurement of GST and UGT activities used compounds that are substrates acted on by multiple forms. Therefore, these assays integrate the response of a number of different isozymes of GST and UGT to the TCDF treatment. Other studies in fish have found no change, induction, or suppression of activity (phenobarbital, PCB, benzo-a-pyrene (Collier and Varanasi, 1991); 3- 
methylcholanthrene (Taysse et al., 1998); 2,3,7,8-TCDD (Hektoen et al., 1994));

frequently the response of GST does not correspond to the response of UGT (Pangrekar and Sikka, 1992; Hitchman et al., 1995; Koponen et al., 1997).

The differences, in inducibility and relative activity between sites, between experiment 1 (female study) and experiment 2 (male study) may have resulted in part from differences in the time of year at which the experiments were done and the resulting reproductive condition of the fish. Experiment 1 was done in early May, at the beginning of the spawning season, when the fish were gravid. Both GST and UGT activity can be affected (either up- or down-regulated) by hormonal expression in mammals ((Igarashi et al., 1984), steroid hormones; (Masmoudi et al., 1996), thyroxin) and in fish (Koivusaari et al., 1981; Sikoki et al., 1989; Leaver and George, 1996). Experiment 2 was conducted in early March, prior to the beginning of the spawning season, with gonadally regressed fish.

A potential complication in both experiments is the remaining PCB body burden of the fish from NBH. The sequence of a plaice GST contains four putative antioxidant response elements (ARE) (Leaver and George, 1996) and GST activity in these fish can be induced by exposure to epoxides (Leaver et al., 1992). In mammals one proposed pathway for GST induction is the production of reactive oxygen species (ROS) which can then either directly or indirectly induce the expression of GST via the AREs. The inefficient metabolism of some PCBs can result in the production of ROS in fish (Schlezinger and Stegeman, 1999). It is possible that the GST activity in gonadally regressed NBH male fish may be moderately induced by the PCB body burden in these fish either directly or indirectly via the production of epoxides or ROS. The TCDF treatments then resulted in greater induction. In a small experiment using gonadally regressed, male, lab-reared offspring of fish from NBH, which had very low PCB body burdens, no GST induction was seen, supporting the hypothesis that the PCBs in the feral adult males affected the expression of GST (Ch. 4). 


\section{In Vitro Studies}

The use of primary hepatocyte cultures allowed us to evaluate a wider range and higher concentrations of inducers than was possible in vivo. Importantly, we were able to obtain induction of CYP1A1 expression in hepatocytes from NBH fish. The NBH hepatocyte cultures were less sensitive to CYP1Al induction than were the SC hepatocytes, 14-fold when treated with TCDD and 3-fold when treated with BNF. A similar difference in responsiveness to halogenated versus non-halogenated compounds was found in tomcod from the Hudson River (Wirgin et al., 1992), which are environmentally exposed to PCBs. CYP1A mRNA in depurated Hudson River tomcod was highly induced by treatment with BNF (15-fold) but not by treatment with PCBs ( 0.85 to 1.5 -fold). There are significant levels of PAHs present in NBH sediment (130 ppm, Lake, 1995), which could potentially induce CYP1A1 expression in NBH killifish. The high concentrations of PCBs also present may inhibit this induction.

There was no significant difference in the maximal EROD activity achieved in primary hepatocytes from NBH or SC fish. This differs from the results of Nacci etal (Nacci et al., 1999), who reported that intact Fundulus embryos from NBH fish had significantly lower maximal EROD activities than embryos from a reference population (West Island). The maximal EROD activity reported in the Nacci study was estimated from a nonlinear least-squares regression curve based on only three doses of inducer. This discrepancy may have resulted from an underestimation of the maximal EROD activity in the NBH embryos due to limits on the maximal dose it is possible to achieve in waterborne exposures.

\section{Mechanism of Resistance}

These results provide some insight into the nature of the resistance in NBH Fundulus. The induction of CYP1A1 in the hepatocytes from NBH fish indicates that these fish still have a functional AhR pathway. The difference in the degree of resistance to halogenated and non-halogenated compounds is a further indication that the poor response 
of fish from NBH to AhR agonists is a function of a change in the sensitivity of the AhR pathway, as opposed to a loss of function in this pathway. This differential resistance (to halogenated versus non-halogenated compounds) is also suggestive of a possible change in the AhR protein, perhaps in the ligand binding domain. Another possibility is that a separate receptor, possibly related to the 4S PAH binding protein found in mammals (Raha et al., 1990; Peryt et al., 1992) and fish (Barton and Marletta, 1988), is responsible for some, if not all, of the response to non-halogenated compounds.

The differential sensitivity of $\mathrm{NBH}$ and $\mathrm{SC}$ fish is consistent with a possible difference in the ligand-binding affinity of their respective AhRs. Such a difference could occur through a mutation in a single base in an $\mathrm{AhR}$ gene. Evidence for this comes from studies in the DBA/2 mouse strain, in which a single amino acid difference in the AhR ligand binding domain - resulting in a lower affinity $\mathrm{AhR}$ - is responsible for the reduced sensitivity to polycyclic aromatic hydrocarbons (PAH) and HAHs in these mice as compared to strains with a high-affinity AhR allele (Poland, 1994) provides support for the possibility of a mutation in the Fundulus AhR that would result in resistance. This would not have to be a novel mutation, but could also result from selection pressure favoring fish with lower affinity AhRs. The variability in inducibility in reference populations suggests that AhRs with varying affinities may exist in Fundulus populations.

Mutations (either new or pre-existing) in the some of the other components of the AhR pathway (ARNT, (Wilson et al., 1997); CYP1A1, (Peterson et al., 1986)) as well as in other parts of the AhR (Hankinson, 1983; Pohjanvirta et al., 1998) that result in resistance to HAHs and PAHs can also be found in other mammalian systems and are also possible explanations for the resistance seen in Fundulus from NBH. The expression of a mutant form of CYP1A1 in fish from NBH is unlikely due to the close agreement of the maximal EROD activities between the two sites, as well as the general agreement of the different measures of CYP1A1 expression both within and between sites. Finally, the expression of two forms of the AhR in Fundulus (Hahn et al., 1997) may provide for an additional mechanism of resistance. If one of these receptors either normally or as the 
result of a mutation can bind ligand without altering gene expression, then an increase in the expression of this receptor relative to the second $A h R$ may also result in decreased sensitivity to AhR agonists.

There are also non-genetic mechanisms of resistance to $\mathrm{AhR}$ agonists that can be induced in mammalian species following prolonged or repeated exposures to AhR agonists. AhR binding to DNA can be down-regulated following exposures to AhR agonists (Reick et al., 1994; Pollenz, 1996). This down-regulation requires expression of an inducible protein. This protein is probably a homolog of the AhR repressor that has been identified in mice (Mimura et al., 1999). However, results from studies using labreared offspring of NBH fish (Ch. 4) and second generation embryos from NBH fish (Nacci et al., 1999) showed that the resistance found in fish from NBH is heritable, strongly suggesting that a genetic mechanism is responsible for this resistance. The high degree of individual variability in inducibility seen in reference populations of Fundulus suggests that there was sufficient genetic variability present in the $\mathrm{NBH}$ population prior to the introduction of the PCBs to allow for selection for one or more of these mechanisms (alteration in AhR, ARNT, or CYP1A1).

Multiple populations of Fundulus resistant to AhR agonists have been reported, but in each case there are distinct features of the phenotype of resistance. For example, CYP1A1 expression in the liver of control fish from NBH was not significantly different from expression in the liver of control fish from SC. But in Fundulus from a resistant population in Newark, NJ, environmentally exposed to TCDD, CYP1A1 expression in untreated fish was significantly higher than in reference fish in the same study (Prince, 1995b). In both populations (NBH and NJ) CYP1A1 expression is not induced following treatment of whole fish with a halogenated compound (TCDF or TCDD respectively).

Another resistant population of Fundulus has been identified in a creosote contaminated site on the Elizabeth River (VA) (Van Veld and Westbrook, 1995). These fish again demonstrated a lack of CYP1A1 inducibility following exposure to a known CYP1A1 inducer, in this case 3-methylcholanthrene, a non-halogenated compound. 
However, CYP1A1 in the Elizabeth River fish was environmentally induced in both hepatic and extrahepatic tissues at some times of the year. The variability in resistance phenotypes among different populations of chemically impacted Fundulus suggests such resistance may occur through many different mechanisms. The actual mechanism(s) present in any one site would therefore depend predominantly on the type of chemical present and the inherent genetic variability of the initial population.

\section{Conclusions}

We have now shown that fish from NBH are resistant to CYP1A1 induction by halogenated and (to a lesser degree) non-halogenated compounds. This resistance is pretranslational, systemic, and persistent. While the mechanism of this resistance is not yet known, the presence of a functional CYP1A1 protein and the difference in sensitivity to halogenated versus non-halogenated compounds suggests that a change in the AhR signal transduction pathway, possibly in the AhR itself, is responsible for the decreased sensitivity in NBH Fundulus. Future research will continue to explore the nature and mechanism of this resistance. 
Chapter 4: HERITABILITY OF DIOXIN RESISTANCE IN FUNDULUS

HETEROCLITUS FROM NEW BEDFORD HARBOR, MA.

Introduction 134

Materials and Methods 136

Materials 136

Fundulus Collection and Maintenance $\quad 139$

Experiment 1: $\quad 136$

Fundulus Treatments 136

Microsome and Cytosol Preparation $\quad 137$

Enzyme Activity Assays 137

$\begin{array}{ll}\text { Protein and RNA Blotting } & 138\end{array}$

$\begin{array}{ll}\text { IHC } & 139\end{array}$

PCB Analysis $\quad 140$

$\begin{array}{ll}\text { Experiment } 2 & 140\end{array}$

Fundulus Treatments $\quad 140$

Homogenate Preparation 141

${ }^{3} \mathrm{H}-\mathrm{TCDD}$ Measurements 141

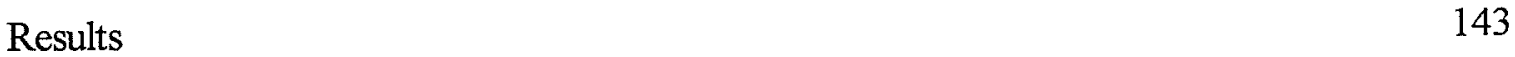

$\begin{array}{ll}\text { Experiment } 1 & 143\end{array}$

Characteristics of $\mathrm{NBHF}_{1}$ Fish 143

CYP1A1 Activity and Expression 143

GST and UGT Activity $\quad 150$

PCB Analysis $\quad 150$

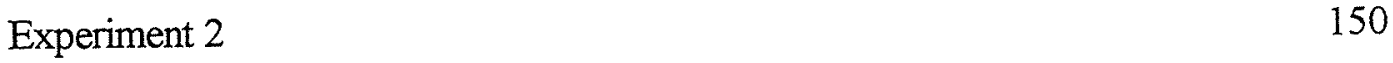

Characteristics of SC and NBH F Fish $\quad 150$

CYP1A1 Activity and Expression $\quad 151$

$\begin{array}{ll}\text { TCDD Distribution } & 160\end{array}$

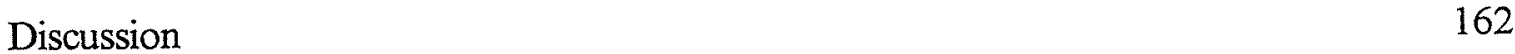




\section{Introduction}

It has long been known that exposure to some halogenated aromatic hydrocarbons $(\mathrm{HAH})$ and polycyclic aromatic hydrocarbons $(\mathrm{PAH})$ can induce a suite of responses through interaction with the aryl hydrocarbon receptor ( $\mathrm{AhR}$ ) signal transduction pathway (reviewed in (Swanson and Bradfield, 1993)). Compounds that act through the AhR pathway bind to the AhR resulting in the release of heat shock protein 90 (HSP90). The AhR then migrates to the nucleus where it binds to the AhR nuclear translocator (ARNT). This complex can then bind to specific regulatory sequences (called dioxin responsive elements, DREs) and alter gene transcription. Some of the genes affected include phase I and phase II xenobiotic metabolizing enzymes, such as cytochrome P450 1A1 (CYP1A1) In studies using AhR knock-out mice, interaction with the AhR has been linked to virtually all of the toxic effects of these compounds (Fernandez Salguero et al., 1996).

A number of studies have shown that long-term or repeated exposures to high concentrations of AhR agonists can result in a decrease in the responsiveness of an individual organism or population of organisms (reviewed in (Hahn, 1998)). Resistance to CYP1A1 induction has been found in populations of killifish, Fundulus heteroclitus, exposed to dibenzo-p-dioxins (Prince, 1995b; Elskus et al., 1999), PCBs (Ch. 2 \& 3; (Nacci et al., 1999), and creosote (PAHs)(Van Veld and Westbrook, 1995). The mechanism(s) of this resistance may have implications for biomonitoring efforts, remediation efforts, as well as the ability of these populations to respond to additional or novel stresses.

In the previous chapters, we reported that killifish (Fundulus heteroclitus) resident in New Bedford Harbor (NBH), MA, a federal Superfund site heavily contaminated with PCBs, showed resistance to induction of CYP1Al following both environmental and laboratory exposures to HAHs compared to killifish from a reference site (Scorton Creek, MA, SC) (Ch. 2 \& 3). This was also in contrast to killifish from moderately contaminated sites where CYP1A1 expression was shown to be induced after environmental exposure to AhR agonists (Burns, 1976; Elskus, 1989). The resistance in NBH killifish was systemic, 
pre-translational and could be overcome in vitro with sufficiently high concentrations of inducers. Further, hepatocytes from NBH fish (compared to hepatocytes from SC fish) were more resistant to induction of CYP1A1 when a halogenated inducer $(2,3,7,8$ tetrachlorodibenzo- $p$-dioxin, TCDD) was used then when a non-halogenated inducer ( $\beta$ naphthoflavone, BNF) was used. These results suggested that the NBH killifish had an alteration in the aryl hydrocarbon receptor (AhR) signal transduction pathway.

To begin to define the mechanism of this resistance, we assessed the heritability of this resistance by examining the induciblity of CYP1Al in lab-reared offspring of NBH killifish. We also asked if the difference in sensitivity to halogenated versus nonhalogenated inducers could be seen in vivo as well as in vitro.

To address these questions we treated 2 year old lab-reared offspring of either $\mathrm{NBH}$ (experiment 1) or SC and NBH (experiment 2) Fundulus with either a halogenated or non-halogenated AhR agonist. The inducibility of CYP1Al expression was examined in a suite of tissues. In addition to CYP1A1 expression in experiment 1, the inducibility of two phase II enzyme families, glutathione-S-transferases (GST) and UDPglucuronosyltransferases (UGT), was examined. To determine if there was any difference in the PCB burdens of $\mathrm{SC}$ and $\mathrm{NBH}$ offspring, the total PCB concentration and congener distributions were determined. To investigate if equivalent doses of inducer were reaching the tissues of interest, the concentration of the halogenated AhR agonist (TCDD) in the liver, gallbladder, carcass, and application site was determined, in experiment 2. 


\section{Materials and Methods}

Materials: Frozen krill was purchased from MidJersey Pet Supply (Carteret, NJ). UPC-10 Ab was purchased from Organon Teknika (West Chester, PA). A universal immunoperoxidase kit (murine) was purchased from Signet Laboratories (Dedham, MA). Permount, SDS and acrylamide were purchased from Fisher (Pittsburgh, PA). ${ }^{3} \mathrm{H}-2,3,7,8-$ TCDD was purchased from ChemSyn (Lenexa, KS). All other chemicals were purchased from Sigma (St Louis, MO).

$\underline{F}$. heteroclitus collection and maintenance: NBH fish were collected by us using baited minnow traps $(6 / 28-6 / 29 / 94, \& 7 / 12-7 / 14 / 95)$ or with unbaited minnow traps $(7 / 23 / 96$ provided by Ken Rocha's group at the EPA Narragansette). SC fish were collected with baited minnow traps $(7 / 18 / 94,8 / 17-8 / 18 / 95,6 / 12 / 96, \& 7 / 25 / 96)$. Fish were maintained in $20^{\circ} \mathrm{C}$ flowing sea water and fed Tetramin ${ }^{\circledR}$ stapleflake and minced krill. In 1994 eggs were fertilized by collecting mature gametes from ripe males and females. Fertilized eggs were collected daily on breeding pads (Scotch $®$ scrub pads covered with plastic screens) during the ' 96 breeding season. After collection the eggs were maintained in sterile petri dishes with sterile filtered sea water until hatching. After hatching the larvae were moved to beakers and fed freshly hatched brine shrimp. The offspring were gradually switched over to the same diet as adults and maintained in 10 gal tanks with $\mathrm{rt}$ recirculating sea water.

\section{Experiment 1:}

F. heteroclitus treatment: Two-year-old male NBH F1 fish were moved to 10 gal. experimental aquaria with $20^{\circ} \mathrm{C}$ flowing seawater 2 days prior to the start of the experiment. The fish were weighed and then dosed intraperitoneally with $50 \mathrm{nmol} / \mathrm{kg}$ $(\mathrm{N}=3)$ 2,3,7,8-tetrachlorodibenzofuran (TCDF) dissolved in corn oil, or corn oil alone $(\mathrm{N}=3$, final $\mathrm{N}=2)$. TCDF solutions were made so fish received 5 ul per $\mathrm{g}$ of fish. After treatment, fish were held for 7 days in static seawater $\left(-20^{\circ} \mathrm{C}\right)$ during which time they were fed only Tetramin ${ }^{\circledR}$ stapleflake. After 7 days fish were killed by cervical transection. Tissues for microsomes were put immediately in ice cold AhR buffer (see below). Tissues 
for immunohistochemistry $(\mathrm{IHC})$ were collected in biopsy cassettes and placed in 10\% neutral buffered formalin. Tissues for RNA were snap frozen in liquid nitrogen and stored at $-80^{\circ} \mathrm{C}$ until RNA preparation. Control carcasses were frozen for analysis of $\mathrm{PCB}$ concentrations. One year later, carcasses from the remaining offspring from the same spawning season ( $3 \mathrm{SC} \mathrm{F}_{1}$ fish) were collected for $\mathrm{PCB}$ analysis.

Microsome and Cytosol preparation: Tissues were homogenized in $9 \mathrm{ml}$ of cold AhR buffer per gram of tissue. AhR buffer consists of $25 \mathrm{mM}$ MOPS (pH 7.5) with $1 \mathrm{mM}$ EDTA, $5 \mathrm{mM}$ EGTA, $0.02 \% \mathrm{NaN}_{3}, 20 \mathrm{mM} \mathrm{Na}_{2} \mathrm{MoO}_{4}, 10 \%$ (v:v) glycerol, $1 \mathrm{mM}$ dithiothreitol, plus protease inhibitors (20 uM TLCK, $5 \mu \mathrm{g} / \mathrm{ml}$ leupeptin, $13 \mu \mathrm{g} / \mathrm{ml}$ aprotinin, $7 \mu \mathrm{g} / \mathrm{ml}$ pepstatin A, and $0.1 \mathrm{mM}$ PMFS) (Hahn et al., 1994). Tissues were homogenized with a Teflon-glass homogenizer (10 passes); intestines and gills were minced with dissecting scissors prior to homogenization. Homogenates were centrifuged for $10 \mathrm{~min}$ at $750 \mathrm{~g}$ and $10 \mathrm{~min}$ at $12,000 \mathrm{~g}$ at $4^{\circ} \mathrm{C}$. The supernatant was then centrifuged at $100,000 \mathrm{~g}$ for $70 \mathrm{~min}$ at $4^{\circ} \mathrm{C}$. This supernatant (cytosol) was removed and frozen in liquid $\mathrm{N}_{2}$. The pellet (microsomes) was resuspended in TEDG $(0.05 \mathrm{M}$ Tris pH 7.5, $1 \mathrm{mM}$ EDTA, $1 \mathrm{mM}$ dithiothreitol, and $20 \%$ glycerol)(Stegeman et al., 1979) and frozen in liquid $\mathrm{N}_{2}$.

EROD activity: CYP1Al activity was determined using a fluorometric, kinetic assay for EROD activity (Hahn et al., 1993). Assays were run in 48 well plates with $2 u \mathrm{M} 7$ ethoxyresorufin and $1.0 \mathrm{mM} \mathrm{NADPH}$ (final concentrations). Samples were scanned once every minute for 15 minutes. Three replicate well were scanned for each sample. A standard resorufin curve (ranging from 0 to $200 \mathrm{pmol}$ of resorufin) was run with each set of samples. Four replicate wells were scanned for each standard concentration. EROD activity was presented as pmol resorufin per minute per mg total microsomal protein. Samples were run in duplicate. EROD activity in individual wells was linear for at least 10 minutes.

Total protein content: Total microsomal or cytosolic protein was measured fluorometrically (Lorenzen and Kennedy, 1993). 
GSH transferase (GST) activity: The protocol described in Habig et al (Habig et al., 1974) as modified by Van Veld et al (Van Veld et al., 1991) was followed, with modifications. Briefly, GST activity was measured by adding cytosol ( 0.02 to $0.14 \mathrm{mg}$ total protein) to $1 \mathrm{ml}$ of reaction buffer containing $1 \mathrm{mM}$ CDNB, $100 \mathrm{mM}$ Tris- $\mathrm{HCl}$, and $1 \mathrm{mM}$ reduced glutathione and then monitoring the change in absorbance for 1 minute at $344 \mathrm{nM}$. GST activity was expressed as nmol of conjugate produced per minute per mg total cytosolic protein. UDP Glucuronosyl Transferase (UGT) activity: The protocol described by Andersson et al (Andersson et al., 1985) was followed, with modifications. Briefly, microsomes (0.01 to 0.11 mg total protein) were added to $0.5 \mathrm{M} \mathrm{KH}_{2} \mathrm{PO}_{4}$ with $p$-nitrophenol $(2.43 \mathrm{mg} / 50 \mathrm{ml})$ and digitonin $(2 \mathrm{mg} / \mathrm{ml})$, plus or minus UDPGA ( $4 \mathrm{mg} / \mathrm{ml}$ ). This mixture was incubated for $20 \mathrm{~min}$ at RT, shaking in the dark. Then $0.45 \mathrm{ml}$ of $3 \%$ trichloroacetic acid was added and the entire mix centrifuged for $15 \mathrm{~min}$ at $4000 \mathrm{rpm}$. $\mathrm{KOH}(0.05 \mathrm{ml}$ of $5 \mathrm{M})$ was added to $0.375 \mathrm{ml}$ of the supernatant and the absorbance was read at $400 \mathrm{~nm}$. UGT activity was expressed as nmol of conjugate produced per minute per mg total microsomal protein.

Immunoblotting: CYP1A1 protein content was measured with a chemiluminescent western blot assay (Hahn et al., 1996) using the Scup 1-12-3 monoclonal antibody (MAb) (Park et al., 1986). A standard curve (0.025 to 0.5 pmol CYP1A1) using scup microsomes with known CYP1A1 content was used to determine CYP1A1 protein in the NBH and SC samples. Samples were loaded so that they fell within the range of the standard curve. Samples were run either on $6 \%$ to $15 \%$ acrylamide gradient gels or $10 \%$ acrylamide non-gradient gels. Proteins were transferred to nylon membranes and then incubated with non-specific blocking solution ( $\mathrm{S} \& \mathrm{~S} \otimes$ blocking powder). Membranes were then incubated with MAb 1-12-3 (10 ug/ml) for 1 $\mathrm{hr}$, washed 3 times ( 3 water rinses then a $5 \mathrm{~min}$ wash with $100 \mathrm{ml}$ of tris buffered saline (TBS)) and incubated for $1 \mathrm{hr}$ with $2^{\circ} \mathrm{Ab}$ (AP-conjugated goat anti-mouse, Bio-Rad, 1.1:1000 dilution). Finally the membrane was washed 3 times again, exposed to a chemiluminescent substrate and placed on x-ray film (Kodak, AR). Multiple exposures were taken of each blot. RNA preparation and Northern blotting: Total RNA was prepared from frozen tissues using TEL-TEST's RNA STAT- $60^{\mathrm{TM}}$ protocol with modifications. Briefly tissue was homogenized 
in RNA STAT- $60^{\mathrm{TM}}$ ( $1 \mathrm{ml}$ per 50 to $100 \mathrm{mg}$ of tissue) then incubated at RT for $\sim 5 \mathrm{~min}$. Chloroform was added $\left(0.2 \mathrm{ml}\right.$ per ml RNA STAT- $\left.60^{\mathrm{TM}}\right)$, mixed by inverting and incubated for 2 to $3 \mathrm{~min}$ at $\mathrm{rt}$. This mix was then centrifuged for $15 \mathrm{~min}$ at $4^{\circ} \mathrm{C}$ at $12,000 \mathrm{~g}$ and the aqueous portion transferred to a new centrifuge tube. Isopropanol $\left(0.5 \mathrm{ml}\right.$ per $1 \mathrm{ml}$ RNA STAT- $\left.60^{\mathrm{TM}}\right)$ was added, mixed by inverting, and incubated for 5 to $10 \mathrm{~min}$ at RT. This mixture was centrifuged for $30 \mathrm{~min}$ at $4^{\circ} \mathrm{C}$ at $12,000 \mathrm{~g}$. The supernatant was poured off and the pellet was washed with ice cold $75 \%$ ethanol and air dried. The pellet was dissolved in DEPC-treated water and stored at $-80^{\circ} \mathrm{C}$. RNA (10 ug per lane) was run in $1 \%$ agarose, $3.6 \%$ formaldehyde gels, transferred to nylon membrane overnight and probed with ${ }^{32} \mathrm{P}$ labeled $\underline{\mathrm{F}}$. heteroclitus CYP1A1 (Morrison et al., 1998).

Immunohistochemistry (IHC): IHC was done according to the methods of Smolowitz et al (Smolowitz et al., 1991), with modifications. In brief, the deparaffinated and hydrated sections were incubated with normal goat serum for 5 minutes to block nonspecific binding of the secondary antibody. Two 1-hour incubations with $150 u \mathrm{~L}$ of monoclonal antibody 1-12-3 (1.7 ug/ml in PBS/BSA) were done. The specificity of this antibody for CYP1A has been shown previously (Miller et al., 1989). Sections were washed after this step and the two following steps. The sections were incubated with secondary antibody (Goat antimouse IgG, 1/200 dilution) for 20 minutes, followed by peroxidase-linked mouse IgG, also for 20 minutes. Then two 15 minute incubations (150 $u \mathrm{~L}$ each) of color developer (Signet) were performed. Sections were counter-stained with Mayers hematoxylin and mounted in crystalmount. Sections of induced and uninduced scup liver were run with each batch of sections as a positive and negative control, respectively. Matching sections were stained with a nonspecific $\operatorname{IgG}$ (purified mouse myeloma protein, UPC-10, Organon Teknika, West Chester, PA, $1.7 \mathrm{ug} / \mathrm{ml}$ in PBS/BSA) as a negative control. Sections were read blind and scored on two scales, occurrence: 0 (no cells staining) to 3 (all cells staining) and intensity: 0 (no staining) to 5 (very dark red staining). These 2 scores were multiplied for a final score ("staining index") of 0 to 15 . 
PCB analysis: Decapitated, eviscerated carcasses or liver pools were homogenized using a virtishear (Virtis). To improve fluidity of the sample during homogenization, 1-2 $\mathrm{ml}$ of methylene chloride-extracted DI water was added to the tissue. An aliquot of the homogenate was taken for wet weight/dry weight determination prior to being dried with sodium sulfate ( $4: 1$, sodium sulfate:tissue). The dried tissue/sodium sulfate mixture was then pulverized with a mortar and pestle, and soxhlet extracted overnight in a 1:1 mixture of acetone:hexane. This extracted was then exchanged into hexane, spiked with PCB congener IUPAC 143 (Ballschmiter and Zell, 1980) as surrogate standard and layered onto a chromatography column packed with $5 \%$ deactivated alumina $/ 5 \%$ deactivated silica. The sample was eluted with $70 \mathrm{ml}$ of hexane to obtain the PCB-containing fraction, volume reduced, exchanged to $1 \mathrm{ml}$ in heptane and injected onto a gas chromatograph (HP5890 SERIES II) fitted with a $30 \mathrm{~m} \mathrm{db}-5$ column and an electron capture detector. Octachloronaphthalene was added just prior to injection to determine recoveries. Total PCB content was measured as a sum of 45 congeners, quantified using a standard mixture of AROCLORS 1232, 1248, and 1262 (25:18:18). Values presented are corrected for recovery. Mean recovery was $57 \%(\min \sim 30 \%, \max \sim 80 \%)$

\section{Experiment 2:}

$\underline{F}$. heteroclitus dosing: Male and female two-year-old SC and NBH F1 fish were weighed prior to being exposed by abdominal absorption (Prince, 1995a) to a $1 \mathrm{ng} / \mathrm{g}$ or $10 \mathrm{ng} / \mathrm{g}$ dose of ${ }^{3} \mathrm{H}-2,3,7,8$-tetrachlorodibenzo-p-dioxin (TCDD) (activity $7.41 \times 10^{13} \mathrm{dpm} / \mathrm{nmol}$ TCDD) dissolved in DMSO, a $10 \mathrm{ug} / \mathrm{g}$ or $100 \mathrm{ug} / \mathrm{g}$ dose of $\beta$-naphthoflavone (BNF) dissolved in DMSO, or DMSO alone. Treatment solutions were made so fish received $5 \mathrm{ul}$ per $g$ of fish. The dosing of fish was spread over 2 days with 3 to 9 fish treated per group per site. The fish were held for 3 days after treatment during which time they were not fed.

After treatment fish were placed in $1 \mathrm{~L}$ beakers with seawater and constant air; no more than 3 fish were placed in any one beaker. After 3 days fish were killed by cervical transection. There was poor survival of fish over the first 24 hours after treatment. Only 
fish which were alive and swimming normally at the end of the 3 days were used in the analyses. Final Ns are presented in Table 4. Prior to tissue collections the abdomen (the application site, defined as the white area on the ventral side of the fish) of each fish was removed for determination of ${ }^{3} \mathrm{H}$-TCDD concentration. Intact gallbladders were collected prior to removal of the liver for determination of ${ }^{3} \mathrm{H}-\mathrm{TCDD}$ concentration. Livers were put immediately in ice cold TEDG buffer (see below). Tissues for IHC (heart, kidney, intestine, gill, gonad, spleen and brain) were collected in biopsy cassettes and placed in $10 \%$ neutral buffered formalin. The eviscerated carcass was saved for determination of ${ }^{3} \mathrm{H}$ TCDD concentration.

The methods for IHC and to determine EROD activity and total protein content were the same as in experiment 1.

Homogenate preparation: Tissues were homogenized in $1 \mathrm{ml}$ of ice cold TEDG $(0.05 \mathrm{M}$ Tris pH 7.5, $1 \mathrm{mM}$ EDTA, $1 \mathrm{mM}$ dithiothreitol, and 20\% glycerol) (Stegeman et al., 1979) per $g$ of tissue. Tissues were homogenized with a Teflon-glass homogenizer (10 passes). After homogenization samples were frozen in $\mathrm{LN}_{2}$.

${ }^{3}$ H-TCDD Concentration: Carcasses and abdominal sections (application site) were digested in $1 \mathrm{M} \mathrm{NaOH}$ (1ml / $200 \mathrm{mg}$ tissue wt)(Prince, 1995a). Gallbladders were bleached with $\mathrm{H}_{2} \mathrm{O}_{2}$, then glacial acetic acid was added to counteract interference of $\mathrm{H}_{2} \mathrm{O}_{2}$ with scintillation counting. Scintillation fluid $(10 \mathrm{ml})$ was added to $1 \mathrm{ml}$ of carcass digest, $30 \mathrm{ul}$ of liver homogenate, or all of the application site digest or gallbladder and the disintegrations per min (DPM) determined.

Curve fit: Graphic analysis of the EROD activity in SC livers relative to the measured liver ${ }^{3} \mathrm{H}-\mathrm{TCDD}$ concentration showed that EROD activity reached a plateau at the higher TCDD concentrations. Therefore, this data was fitted to a modified hyperbolic function (DeVito et al., 1997):

$$
\mathrm{y}=\mathrm{E}_{0}+\left\{\left(\mathrm{E}_{\mathrm{m}}-\mathrm{X}^{\mathrm{n}}\right) /\left(\mathrm{b}^{\mathrm{n}}+\mathrm{X}^{\mathrm{n}}\right)\right\}
$$


where $y$ is the EROD activity, $E_{m}$ is the maximal EROD activity, $E_{0}$ is the basal EROD activity, $\mathrm{X}$ is the dose, $\mathrm{b}$ is the EC50, and $\mathrm{n}$ is a shaping parameter that allows the curve to be hyperbolic $(n<1)$ or sigmoidal $(n>1)$. 


\section{$\underline{\text { Results }}$}

Previous studies showed that feral NBH Fundulus appear to be resistant to induction of CYP1A1 following either environmental or laboratory exposures to AhR agonists. In these studies we examined the heritability of this resistance by exposing offspring of fish collected in $\mathrm{NBH}\left(\mathrm{F}_{1}\right.$ fish) to 3 different $\mathrm{AhR}$ agonists.

\section{Experiment 1:}

In a pilot study to assess the responsiveness of $\mathrm{NBH} \mathrm{F}_{1}$ fish, a small of $3^{+}$-year-old male fish were treated with TCDF. There was no significant difference in the mean body weight, LSI, or protein yield between the control and TCDF treated fish (Table 1). The mean GSI of the control fish was significantly higher than that of the TCDF treated fish $(p<0.05)$. Despite this difference in GSIs all of the fish had gonads that appeared to be regressed.

CYP1A1: All three measures of CYP1A1 expression gave the same results (Table 1). There was no significant difference in mean EROD activity between the fish in the control and TCDF groups. Mean CYP1A1 protein levels were not significantly different in the control fish versus the TCDF treated fish. And finally there was no significant difference in CYP1A1 mRNA levels between fish from the two groups. IHC analysis revealed that this resistance was systemic. CYP1A1 protein was not induced in any cell type of any tissue examined (Table 2). Only the proximal tubules of the kidney expressed any appreciable levels of CYP1A1, and no treatment-related difference was seen in the amount of CYP1A1 in these cells. 
Table 1: Body weight, liver somatic index $(\mathrm{LSI})^{1}$, gonad somatic index (GSI), microsomal yield, CYP1A1 expression and Phase II enzyme activities in $\mathrm{NBH} \mathrm{F}_{1}$ fish treated with TCDF (experiment 1).

\begin{tabular}{|l|l|l|}
\hline \multicolumn{1}{|c|}{ TCDF (nmol/kg) } & \multicolumn{1}{c|}{0} & \multicolumn{1}{c|}{50} \\
\hline Body Weight (g) & $2.38 \pm 0.004$ & $3.31 \pm$ \\
\hline LSI (\%) & $2.86 \pm 0.84$ & $2.50 \pm 0.60$ \\
\hline GSI (\%) & $1.20 \pm 0.061$ & $0.67 \pm 0.081^{*}$ \\
\hline Microsomal Yield (mg / g) & $9.28 \pm 1.00$ & $9.13 \pm 0.65$ \\
\hline EROD (pmol / min / mg) & $149 \pm 25$ & $581 \pm 117$ \\
\hline CYP1A1 Protein (pmol/mg) & $0.0031 \pm 0.0014$ & $0.0068 \pm 0.0013$ \\
\hline CYP1Al mRNA (integrated density / ug total RNA) & $6.3 \pm 3.6$ & $10.8 \pm 1.6$ \\
\hline GST (nmol / min / mg) & $376 \pm 3$ & $369 \pm 15$ \\
\hline UGT (nmol/min / mg) & $4.2 \pm 2.2$ & $5.4 \pm 0.6$ \\
\hline
\end{tabular}

${ }^{1}$ The LSI is the ratio of the total weight of the liver compared to the total weight of the fish multiplied by 100 . The GSI is the ratio of the total weight of the gonad compared to the total weight of the fish multiplied by 100 . * significantly different from control $(p<0.05$, 2-tailed t-test) 
Table 2: IHC analysis of CYP1A1 protein in NBH offspring treated with TCDF (experiment 1)

\begin{tabular}{|c|c|c|}
\hline & \multicolumn{2}{|c|}{$\begin{array}{l}\text { CYP1A1 Protein } \\
\left(\mathrm{N}^{2}\right)\end{array}$} \\
\hline Tissue/TCDF dose & $0(\mathrm{nmol} / \mathrm{kg})$ & $50(\mathrm{nmol} / \mathrm{kg})$ \\
\hline \multicolumn{3}{|l|}{ Liver } \\
\hline Hepatocytes & $\mathrm{np}$ & $0.0 \pm 0.0(2)$ \\
\hline Vasc. endothel. & $\mathrm{np}$ & $3.5 \pm 0.5(2)$ \\
\hline \multicolumn{3}{|l|}{ Gill } \\
\hline Epithelium & $0.0 \pm 0.0(2)$ & $0.0 \pm 0.0(3)$ \\
\hline Vasc. endothel. & $0.0 \pm 0.0(2)$ & $0.0 \pm 0.0(3)$ \\
\hline \multicolumn{3}{|l|}{ Kidney } \\
\hline Proximal tubules & $9.0 \pm 0.0(2)$ & $7.5 \pm 0.0(3)$ \\
\hline Distal tubules & $0.0 \pm 0.0(2)$ & $0.0 \pm 0.0(3)$ \\
\hline Vasc. endothel & $0.0 \pm 0.0(2)$ & $0.0 \pm 0.0(3)$ \\
\hline \multicolumn{3}{|l|}{ Intestine } \\
\hline Epithelium & $0.0 \pm 0.0(2)$ & $0.0 \pm 0.0(3)$ \\
\hline Vasc. endothel. & $0.0 \pm 0.0(2)$ & $0.33 \pm 0.33(3)$ \\
\hline \multicolumn{3}{|l|}{ Gonad } \\
\hline Vasc. endothel & $0.0 \pm 0.0(2)$ & $0.0 \pm 0.0(3)$ \\
\hline \multicolumn{3}{|l|}{ Heart } \\
\hline Atrium & $\mathrm{np}$ & $1.0 \pm 1.0(2)$ \\
\hline Ventricle & $0.0 \pm 0.0(1)$ & $0.0 \pm 0.0(3)$ \\
\hline
\end{tabular}

${ }^{1}$ CYP1A1 protein was reported as the product of two scores, intensity and occurrence (see methods). ${ }^{2} \mathrm{~N}=$ number of fish in which each cell type was scored. $\mathrm{np}=$ the tissue was not present in the section scored. 
Fig. 4-1: Comparison of $\mathrm{PCB}$ congener distribution in $\mathrm{SC}$ and $\mathrm{NBH}$ offspring from experiment 1 . There was no significant difference in the distribution of congeners between sites. Bars represent the mean of $3(\mathrm{SC})$ or $2(\mathrm{NBH})$ fish. 


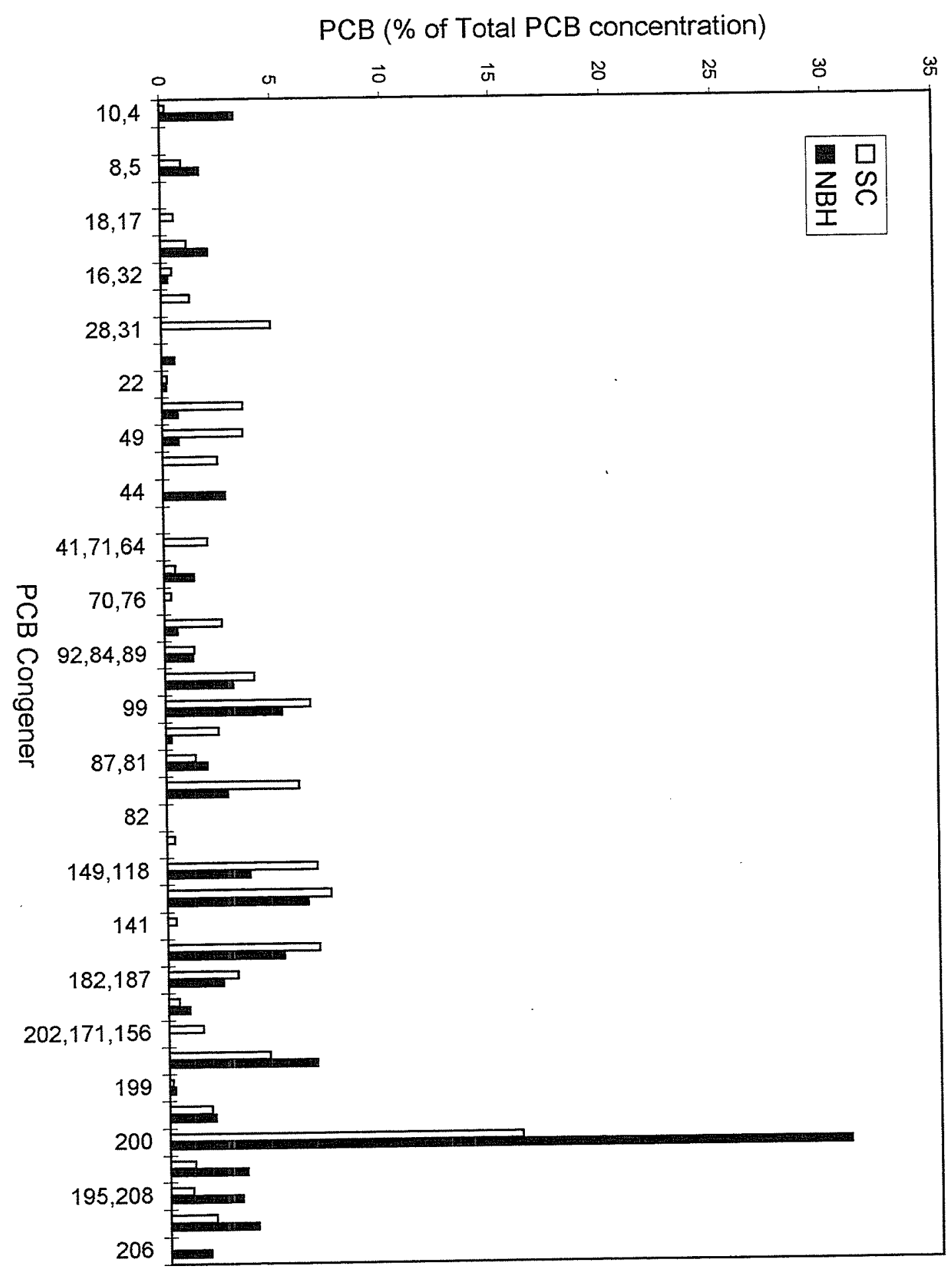


Fig. 4-2: Comparison of the ratio to PCB \#138 of selected congeners in $\mathrm{SC}$ and $\mathrm{NBH} \mathrm{F}_{1}$ fish. Congeners selected all have adjacent unsubstituted carbons on at least one ring except for PCB \#200. Open bars represent SC, striped bars represent NBH. 
Ratio to 138

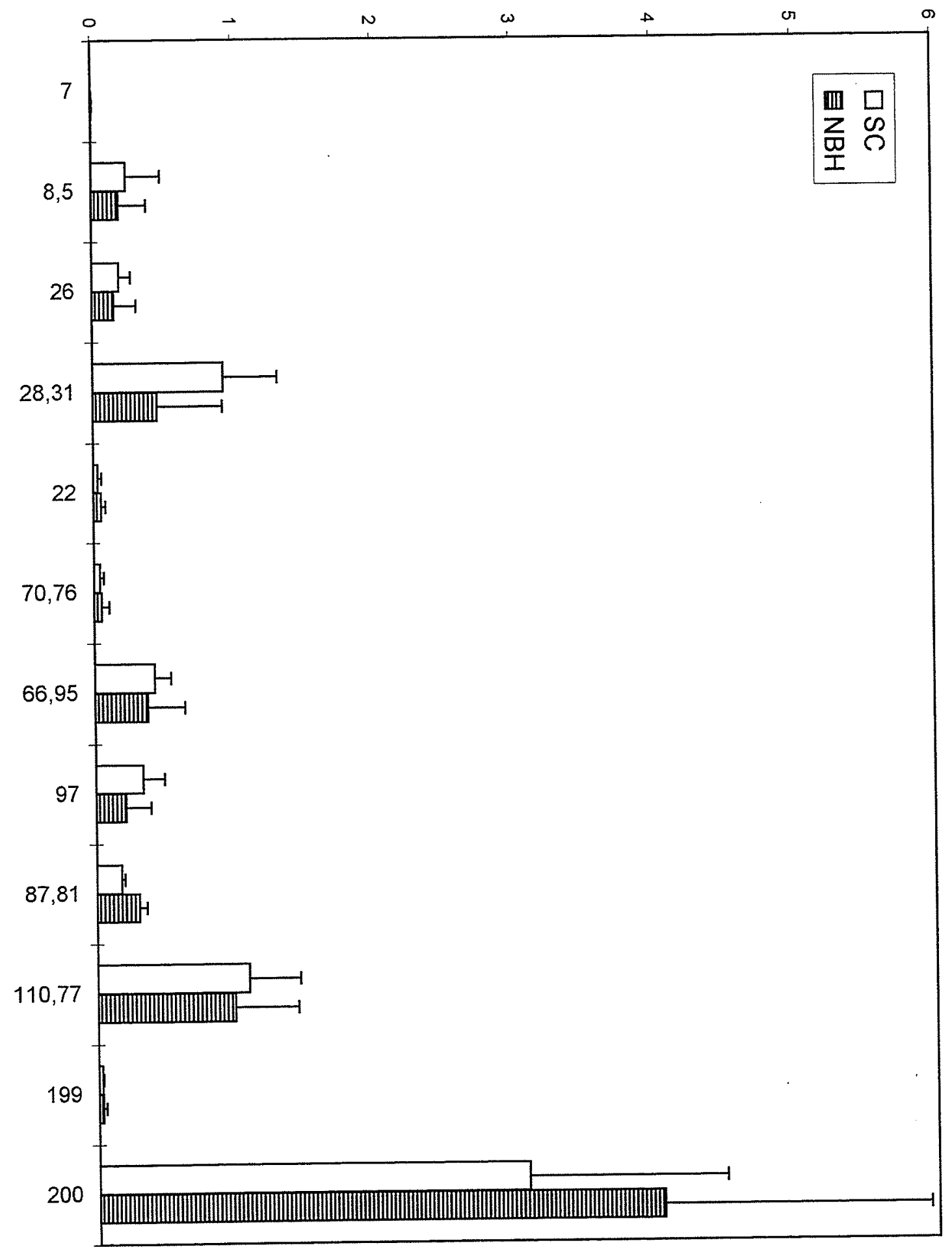


Phase II enzymes: Similar to results from analyses of CYP1A1 expression there was no significant change in the GST activity in the TCDF treated fish compared to the control fish. There was also no significant difference in UGT activity between fish in the control and TCDF treatment groups (Table 2).

PCB Analysis: Both the NBH $(211 \pm 60 \mathrm{ng} / \mathrm{g}$, dry weight $)$ and $\mathrm{SC}(270 \pm 30 \mathrm{ng} / \mathrm{g}) \mathrm{F}_{1}$ fish had total PCB concentrations in the same range as those found in feral SC fish (177 \pm $18 \mathrm{ng} / \mathrm{g}, \mathrm{Ch}$. 2) and much lower than that found in feral NBH fish (272,000 ng / g). The pattern of $\mathrm{PCB}$ congener concentrations were quite similar in offspring from both sites (Fig. 4-1). The peak at congener 200 is the major PCB peak in $\mathrm{F}_{1}$ fish from each site. This peak accounts for $\sim 31 \%$ of the total PCB body burden in the SC fish and $\sim 16 \%$ of the total PCB body burden in the NBH fish. The next largest peak accounts for $\sim 7 \%$ and $\sim 6 \%$ of the total PCB body burden in SC and NBH fish, respectively. This congener makes a relatively minor contribution to the $\mathrm{PCB}$ concentration in the carcasses of feral SC ( $0.58 \%)$ and $\mathrm{NBH}(0.06 \%)$ fish. The ratios of selected congeners with adjacent unsubstituted carbons on at least one ring to PCBs \# 138 and 163 are shown in Fig. 4-2. PCB 138 is has a relatively long half life in fish and can be used to as a baseline to assess metabolism of PCBs. There is no significant difference in these ratios between $\mathrm{SC}$ and NBH fish.

\section{Experiment 2:}

For experiment 1 , we did not have sufficient male $S C F_{1}$ fish available to do a parallel induction study in fish from a parental stock that did respond to TCDF. In experiment 2 , a larger population of $\mathrm{F}_{1}$ fish derived from either $\mathrm{SC}$ or $\mathrm{NBH}$ parents was available, allowing for comparisons of CYP1A1 inducibility to be made. For this experiment, fish were treated with ${ }^{3} \mathrm{H}-\mathrm{TCDD}$ ( 2 doses) or BNF.

There was no significant difference in body weight between sites or doses (Table 3). The apparent trend of decreasing body weight with increasing TCDD dose in the SC 
offspring is an artifact of the random selection of fish for each dose and was present prior to the fish being treated (not shown). There was no significant difference in the liver somatic index (LSI) or in the gonad somatic index (GSI) between site or treatment. The large variability in the GSI values of the NBH control and treated ( $1 \mathrm{ng}$ TCDD / g) fish was due to the presence of 1 ripe female in each of these groups.

Table 3: Mean body weight, LSI, and GSI for SC and NBH lab-reared offspring (experiment 2).

\begin{tabular}{|l|c|c|c|c|c|c|c|c|}
\hline & \multicolumn{2}{|c|}{$\begin{array}{c}\text { Body Weight } \\
\text { (g) }\end{array}$} & \multicolumn{2}{c|}{$\begin{array}{c}\text { LSI } \\
(\%)\end{array}$} & \multicolumn{2}{c|}{$\begin{array}{c}\text { GSI } \\
(\%)\end{array}$} & \multicolumn{2}{c|}{$\begin{array}{c}\text { Protein Yield } \\
\text { (mg/g) }\end{array}$} \\
\hline Inducer & SC & NBH & SC & NBH & SC & NBH & SC & NBH \\
\hline DMSO & $2.37 \pm$ & $1.74 \pm$ & $1.27 \pm$ & $2.52 \pm$ & $1.05 \pm$ & $3.63 \pm$ & 162.07 & 155.45 \\
control & 0.43 & 0.42 & 0.15 & 0.47 & 0.26 & 2.53 & \pm 16.91 & \pm 7.91 \\
& $(6)$ & $(4)$ & $(6)$ & $(4)$ & $(6)$ & $(4)$ & $(6)$ & $(4)$ \\
\hline $1 \mathrm{ng} / \mathrm{g}$ & $1.65 \pm$ & $1.82 \pm$ & $2.72 \pm$ & $1.97 \pm$ & $0.76 \pm$ & $4.08 \pm$ & 130.96 & 146.15 \\
$\mathrm{TCDD}$ & 0.42 & 0.45 & 0.50 & 0.17 & 0.13 & 2.82 & \pm 21.38 & \pm 10.46 \\
& $(5)$ & $(5)$ & $(5)$ & $(5)$ & $(5)$ & $(5)$ & $(5)$ & $(5)$ \\
\hline $10 \mathrm{ng} / \mathrm{g}$ & $1.51 \pm$ & $1.41 \pm$ & $3.14 \pm$ & $3.02 \pm$ & $1.09 \pm$ & $1.76 \pm$ & $98.88 \pm$ & 125.99 \\
$\mathrm{TCDD}$ & 0.31 & 0.21 & 1.19 & 0.37 & 0.38 & 0.91 & 3.85 & \pm 14.00 \\
& $(4)$ & $(3)$ & $(4)$ & $(3)$ & $(4)$ & $(3)$ & $(4)$ & $(3)$ \\
\hline $10 \mathrm{ug} / \mathrm{g}$ & $1.85 \pm$ & $2.48 \pm$ & $3.07 \pm$ & $2.72 \pm$ & $1.43 \pm$ & $1.23 \pm$ & 116.74 & 124.81 \\
$\mathrm{BNF}$ & 0.20 & 0.43 & 0.48 & 0.17 & 0.50 & 0.36 & \pm 8.54 & \pm 13.04 \\
& $(5)$ & $(4)$ & $(5)$ & $(4)$ & $(5)$ & $(4)$ & $(5)$ & $(4)$ \\
\hline $100 \mathrm{ug} / \mathrm{g}$ & 1.35 & 1.11 & 1.04 & 1.62 & 4.39 & 2.43 & 97.90 & 152.13 \\
$\mathrm{BNF}$ & $(1)$ & $(1)$ & $(1)$ & $(1)$ & $(1)$ & $(1)$ & $(1)$ & $(1)$ \\
\hline
\end{tabular}

${ }^{1}$ The NBH control and $1 \mathrm{ng} / \mathrm{g}$ TCDD groups each contained one gravid fish, without this fish the GSIs for these groups were $1.10 \pm 0.11$ and $1.26 \pm 0.31$ respectively.

CYP1A1:EROD activity was significantly induced in the SC offspring by both the 1 (7.5fold) and $10 \mathrm{ng} / \mathrm{g}$ (13-fold) TCDD treatments, though not by the $10 \mathrm{ug} / \mathrm{g}$ BNF treatment (Table 4). EROD activity in NBH offspring was not significantly induced by any of the treatments. 
Differences in uptake or distribution of TCDD could potentially influence the sensitivity of CYP1A1 inducibility. Therefore, we measured the actual hepatic concentration of ${ }^{3} \mathrm{H}-\mathrm{TCDD}$ and expressed liver EROD activity as a function of this concentration. CYP1A1 activity is higher in the livers of the majority of SC offspring at TCDD concentrations very similar to those in NBH offspring (Fig. 4-3). This difference is more pronounced at the higher TCDD dose where there is no overlap in EROD activities between offspring from each site. The dose-response curve of TCDD in the SC offspring clearly showed a plateau and was fitted to a modified hyperbolic function (DeVito et al., 1997). The EC50 for EROD induction in SC livers determined by this method was $7.6 \mathrm{ng}$ $\mathrm{TCDD} / \mathrm{g}$ liver. There was no plateau in the dose-response curve of TCDD in the NBH fish. This data was therefore not fitted to the hyperbolic function. When both of the doseresponse curves were analyzed by linear regression, the resulting regression lines had significantly different slopes and y-intercepts.

As in feral Fundulus from NBH, the lack of CYP1A1 inducibility by TCDD in $\mathrm{NBH} \mathrm{F}_{1}$ fish was systemic. Using IHC analysis, no significant induction of CYP1A1 protein was found in any of the extrahepatic tissues (heart, kidney, intestine, gill, gonad, spleen, and brain) of NBH offspring treated with either 1 or $10 \mathrm{ng} / \mathrm{g}$ TCDD (Fig. 4-4 A, C, E \& Fig. 4-5). In contrast, there was induction in at least one cell type (vascular endothelium) in each of these tissues in SC offspring (Fig. 4-4 B, D, F \& Fig. 4-5).

IHC analysis also revealed a difference in sensitivity to halogenated (TCDD) versus non-halogenated (BNF) inducers, similar to that seen in primary hepatocytes from feral NBH Fundulus (Ch. 3). Significant induction of CYP1Al protein was seen in the vascular endothelium of the kidney, gill, and brain as well as in the atrium (Fig. 4-4 G \& Fig. 4-5) in NBH offspring treated with BNF but not in NBH fish treated with TCDD. Similar significant induction was seen in the extrahepatic tissues of SC offspring (Fig. 4-4 H \& Fig. 4-5) treated with either TCDD or BNF. 
Table 4: Hepatic ${ }^{3} \mathrm{H}-\mathrm{TCDD}$ concentration and EROD activity in $\mathrm{SC}$ and $\mathrm{NBH} \mathrm{F}_{1}$ fish

\begin{tabular}{|l|l|l|l|l|}
\hline & \multicolumn{2}{|c|}{ Hepatic $^{3} \mathrm{H}-\mathrm{TCDD}$ (ng / g) } & \multicolumn{2}{c|}{$\begin{array}{c}\text { Hepatic EROD Activity } \\
\text { (pmol/min/mg) }\end{array}$} \\
\hline Site/Inducer & $\mathrm{SC}$ & $\mathrm{NBH}$ & $\mathrm{SC}$ & $\mathrm{NBH}$ \\
\hline DMSO Control & 0 & 0 & $57.8 \pm$ & $176 \pm$ \\
& & & 7.87 & $16.7^{*}$ \\
\hline 1 ng. / g TCDD & $7.39 \pm$ & $4.20 \pm$ & $435 \pm$ & $197 \pm$ \\
& 1.10 & 0.78 & $89.3^{* *}$ & 29.31 \\
\hline $10 \mathrm{ng} / \mathrm{g}$ & $37.1 \pm$ & $35.7 \pm$ & $752 \pm$ & $234 \pm$ \\
TCDD & 6.53 & 10.2 & $101^{*}$ & 83.0 \\
\hline 10 ug / g BNF & ND & ND & $77.0 \pm$ & $126 \pm$ \\
& & & 19.4 & 17.9 \\
\hline
\end{tabular}

${ }^{*}$ EROD activity is significantly higher in NBH fish than in SC fish $(\mathrm{p}<0.01,2$-tailed $\mathrm{t}-$ test). ${ }^{*}$ EROD activity is significantly different from control EROD activity in fish from the same site $(p<0.05$, ANOVA). ND: not determined 
Fig. 4-3: EROD activity as a function of measured liver ${ }^{3} \mathrm{H}-\mathrm{TCDD}$ concentrations in SC (solid) and NBH (open) offspring from experiment 2. The dose-response curve for TCDD in NBH fish is significantly different from the dose-response curve in $\mathrm{SC}$ fish $(\mathrm{p}<0.05$, comparison of the slope and y-intercept from linear regressions). The dose-response data for TCDD in SC fish were fitted to a hyperbolic function (solid line). The dose-response data for TCDD in NBH fish were fitted to a simple linear function (dashed line). 


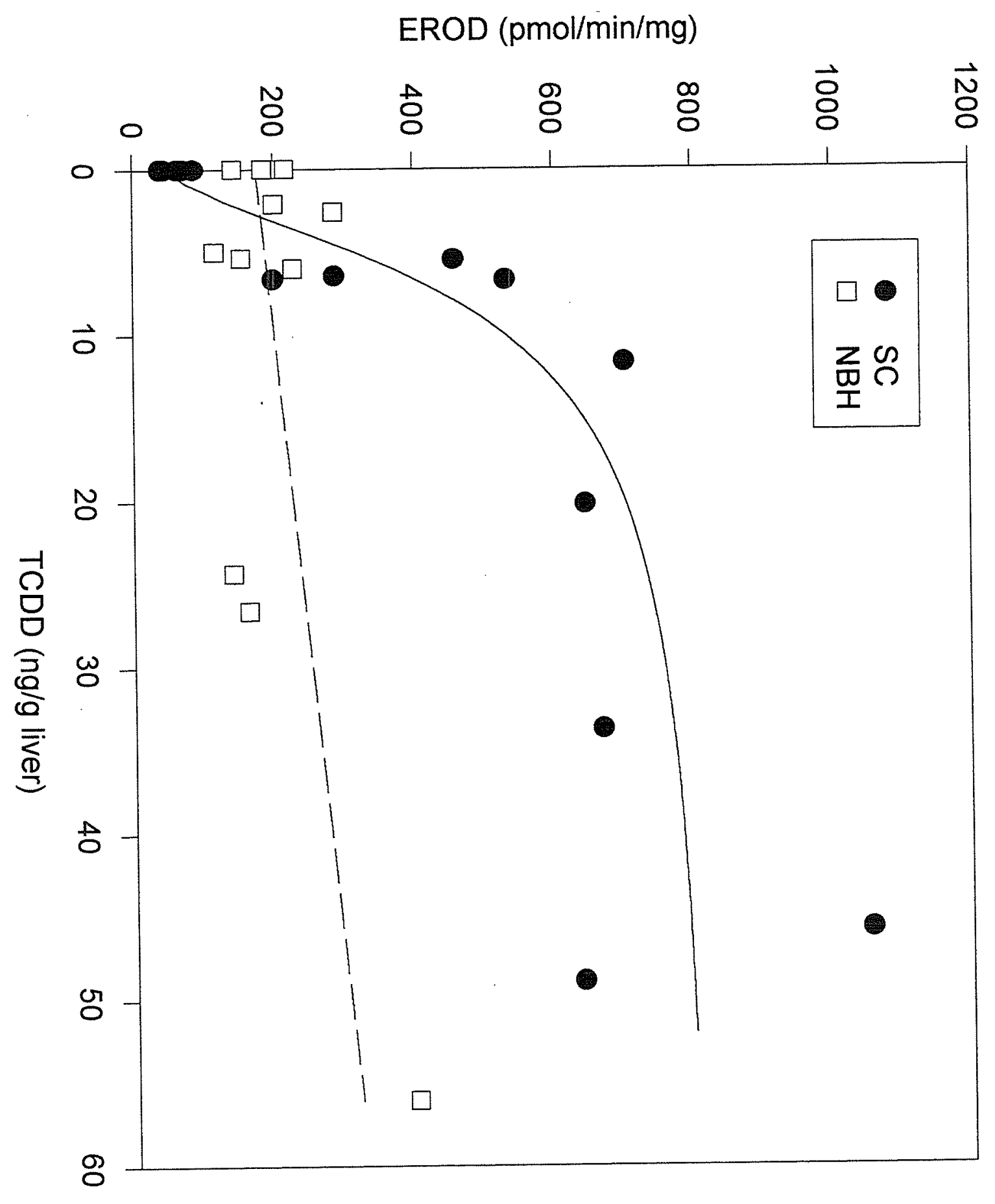


Fig. 4-4: CYP1A1 protein in the atrium of SC and NBH offspring exposed to DMSO, 1 $\mathrm{ng} / \mathrm{g}$ TCDD, $10 \mathrm{ng} / \mathrm{g}$ TCDD, or $10 \mathrm{ug} / \mathrm{g}$ BNF from experiment 2. (A) NBH DMSO (B) SC DMSO (C) NBH 1 ng/g TCDD (D) SC 1 ng/g TCDD (E) NBH 10 ng/g TCDD (F) $\mathrm{SC} 10 \mathrm{ng} / \mathrm{g}$ TCDD (G) NBH $10 \mathrm{ug} / \mathrm{g}$ BNF (H) SC $10 \mathrm{ug} / \mathrm{g}$ BNF. All photos are 200X. 
$\mathrm{NBH}$
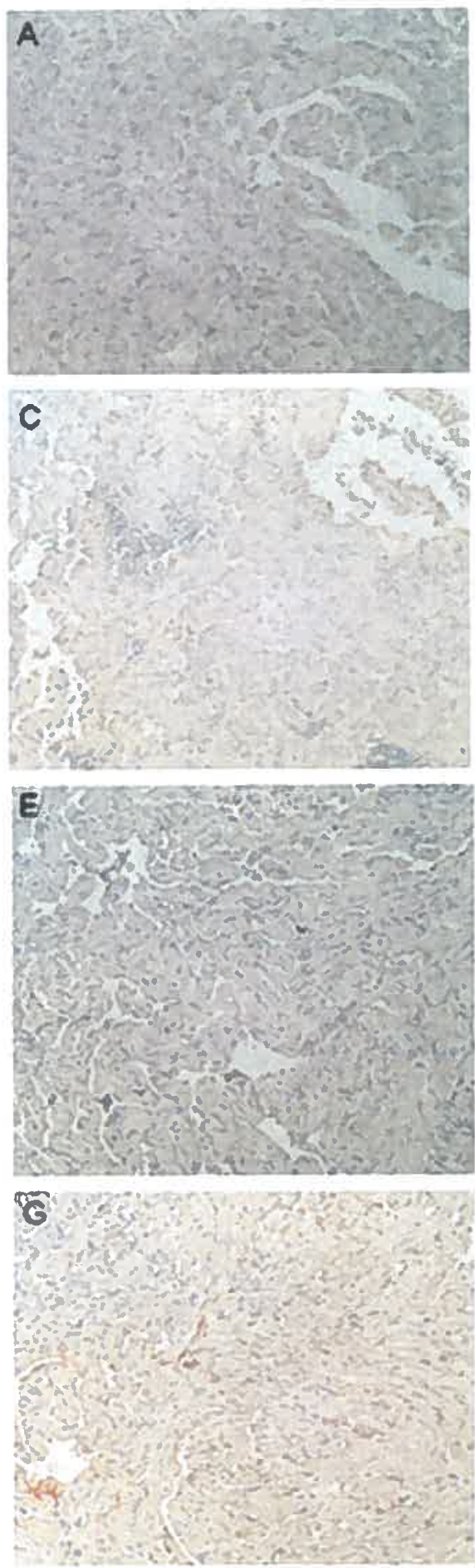

SC
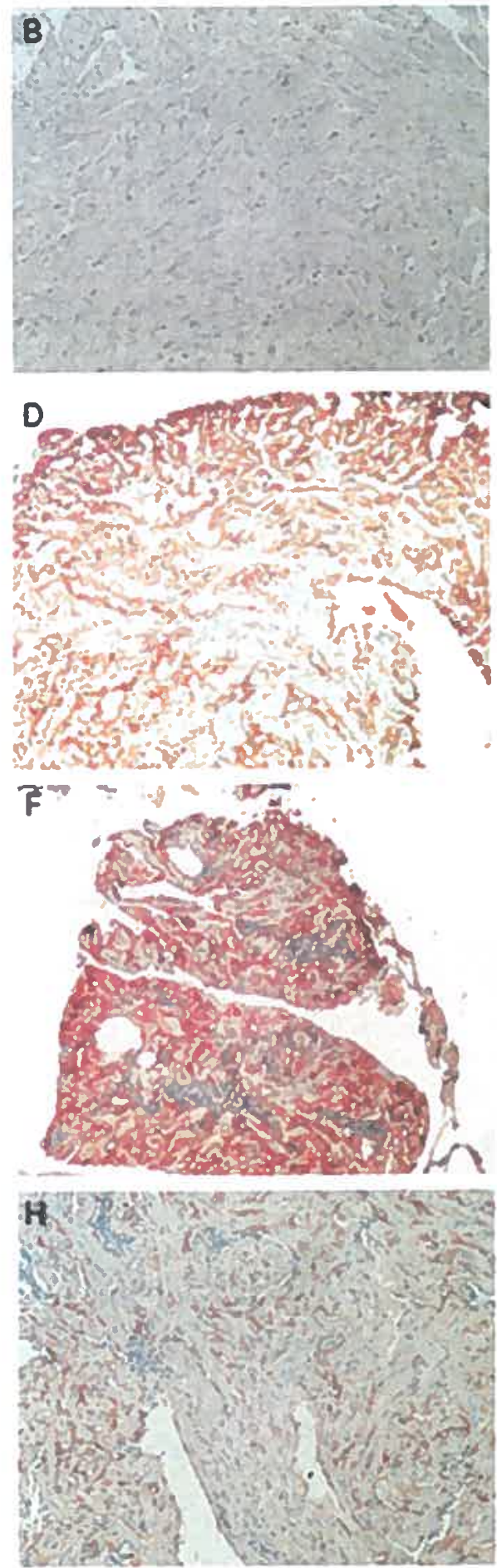
Fig. 4-5: IHC analysis of CYP1A1 expression in extrahepatic tissues (atrium, kidney vascular endothelium and kidney proximal tubules) of SC (A, C) and NBH (B, D) offspring exposed to DMSO, $1 \mathrm{ng} / \mathrm{g}$ TCDD, $10 \mathrm{ng} / \mathrm{g}$ TCDD (A, B), or $10 \mathrm{ug} / \mathrm{g}$ BNF or $100 \mathrm{ug} / \mathrm{g}$ BNF (C, D) (experiment 2). Tissues are scored on two scales: Occurrence (Occ., 0-3) and Intensity (Int., 0-5) the results of these two measurements are multiplied to give a final (Occ. $x$ Int.) score from 0 to 15. Open bars: SC; forward-slashed bars: NBH. ND: no CYP1Al protein was detected (Occ. $\mathrm{x}$ Int. $=0$ ). $\mathrm{N}=1$ for all $100 \mathrm{ug} / \mathrm{g} \mathrm{BNF}$ and for kidney proximal tubules and vascular endothelium from $\mathrm{SC}$ fish treated with $10 \mathrm{ng} / \mathrm{g}$ TCDD. $\mathrm{N}=2$ for all tissues from NBH fish treated with $10 \mathrm{ng} / \mathrm{g}$ TCDD and atria from SC fish treated with $10 \mathrm{ng} / \mathrm{g}$ TCDD and NBH fish treated with DMSO or $1 \mathrm{ng} / \mathrm{g}$ TCDD. $\mathrm{N}=$ 3 for all tissues from NBH fish treated with $10 \mathrm{ug} / \mathrm{g}$ BNF, kidney cells from SC fish treated with $1 \mathrm{ng} / \mathrm{g}$ TCDD and NBH fish treated with DMSO, and atria from SC fish treated with DMSO. $\mathrm{N}=4$ for kidney proximal tubules from $\mathrm{SC}$ fish treated with DMSO and $10 \mathrm{ug} / \mathrm{g} \mathrm{BNF}$ and kidney vascular endothelium from SC fish treated with $10 \mathrm{ug} / \mathrm{g}$ BNF. $\mathrm{N}=5$ for kidney cells from NBH fish treated with $1 \mathrm{ng} / \mathrm{g}$ TCDD, kidney vascular endothelium from SC fish treated with DMSO, and atria from SC fish treated with $10 \mathrm{ug} / \mathrm{g}$ $\mathrm{BNF}$. 


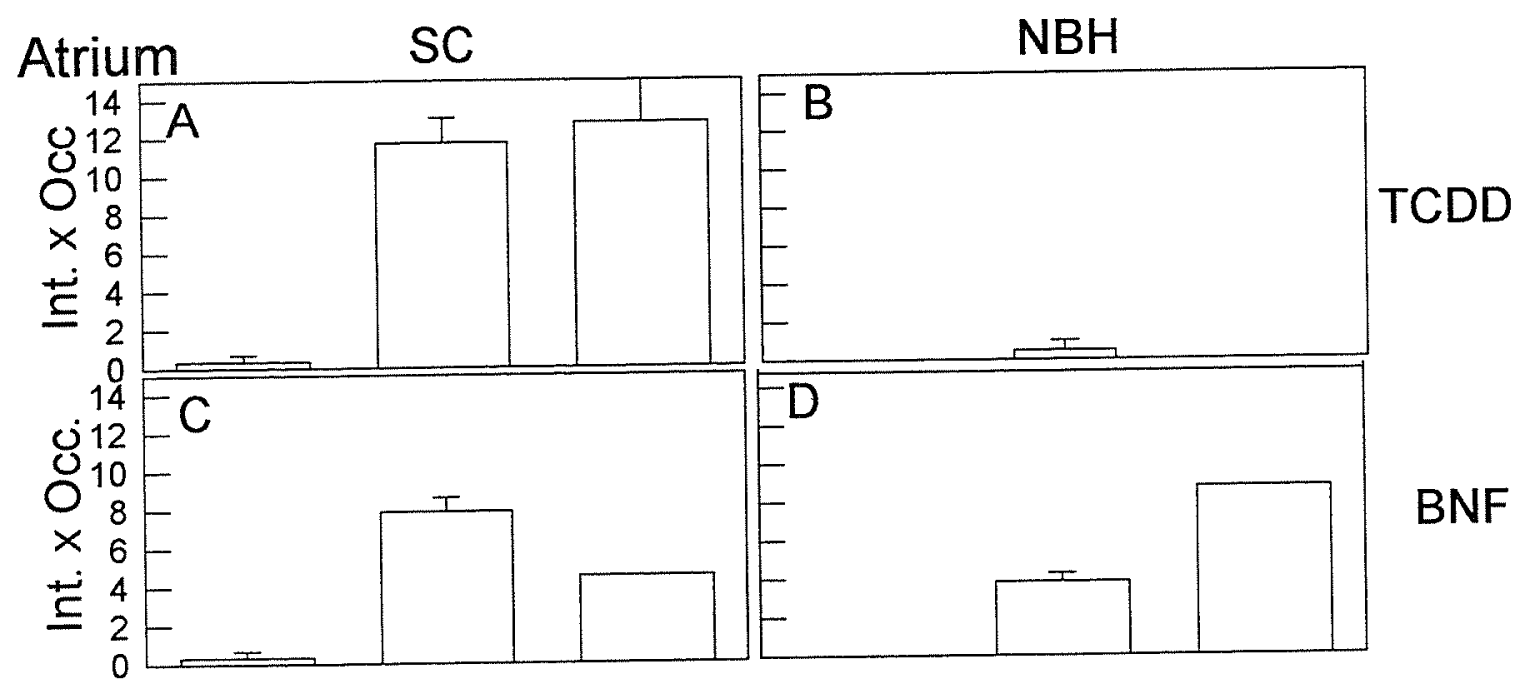

Kidney Vascular Endothelium

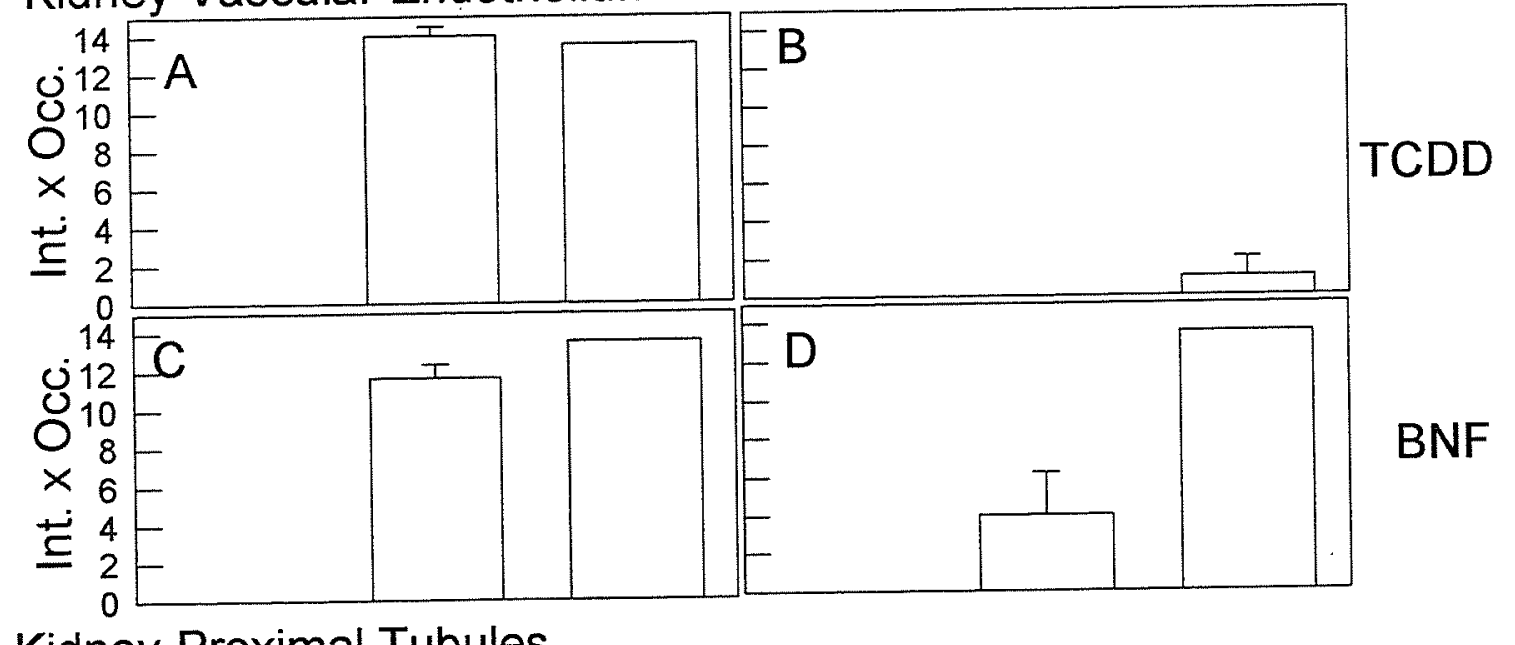

Kidney Proximal Tubules

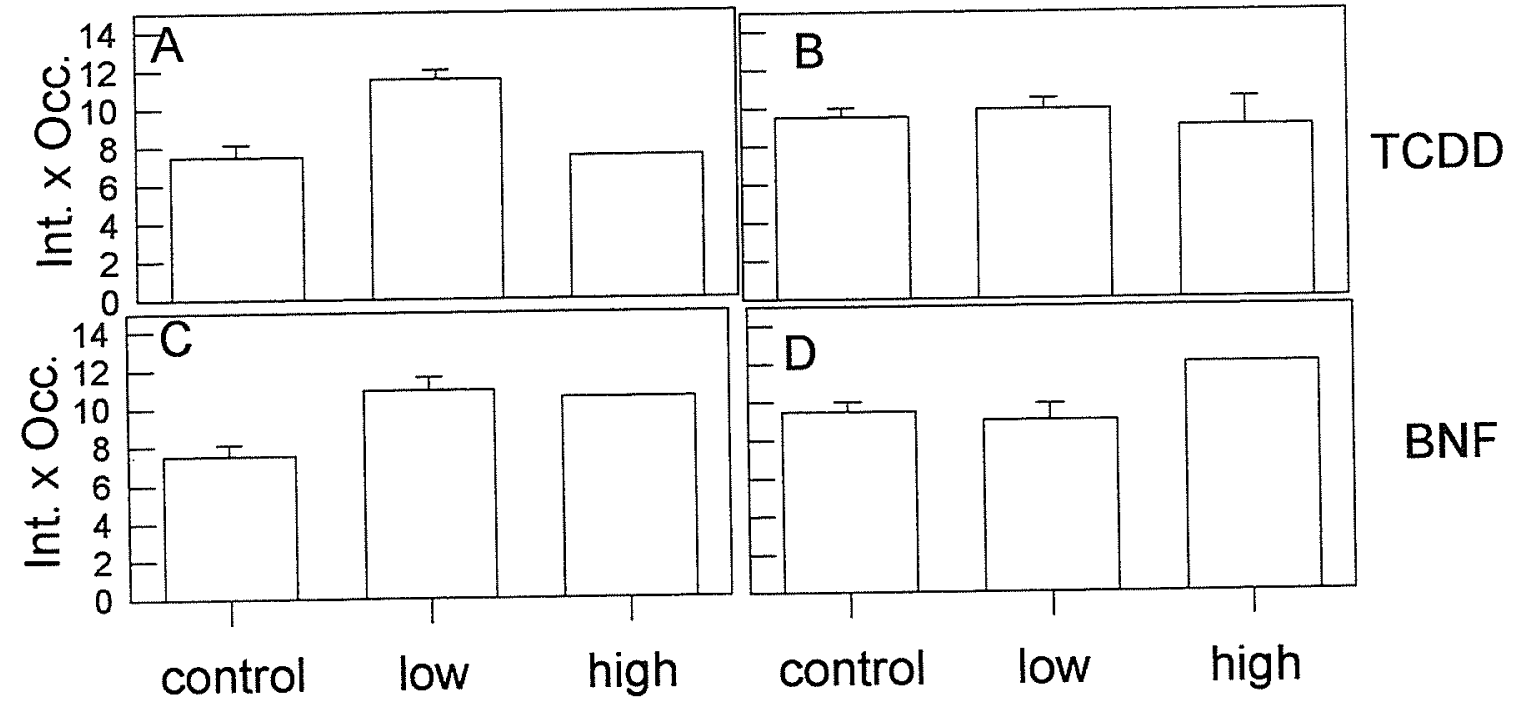


TCDD Distribution: One possible mechanism of resistance that could be present in the NBH killifish population is an alteration in the way HAHs are distributed in the body. Sensitive tissues could be protected from the negative effects of HAHs through preferential sequestration of the HAHs in other tissues. To investigate if there was any difference in the ${ }^{3} \mathrm{H}-\mathrm{TCDD}$ distribution between NBH and SC fish, the concentration of ${ }^{3} \mathrm{H}-\mathrm{TCDD}$ was determined in the liver, bile, carcass, and application site.

In the $1 \mathrm{ng} / \mathrm{g}$ TCDD groups there was no significant difference between $\mathrm{SC}$ and NBH fish in the concentration of TCDD in any of the tissues (carcass, application site, liver, and gallbladder) (Table 5). In fish from both sites TCDD was preferentially concentrated in the liver and bile. In the $10 \mathrm{ng} / \mathrm{g}$ TCDD groups there was a significant difference in the concentration of TCDD in the application site and gallbladder between the SC and NBH fish. The SC fish had significantly more TCDD in the gallbladder and less in the application site than NBH fish. Total recovery was low $\sim 18$ to $40 \%$ but not significantly different between sites. Another $3-4 \%$ of the TCDD could be estimated to be in the extrahepatic tissues, assuming equivalent concentrations as found in the liver of the same fish. 
Table 5: Hepatic and Extrahepatic ${ }^{3} \mathrm{H}$-TCDD concentrations in SC and NBH $F_{1}$ fish

\begin{tabular}{|c|c|c|c|c|}
\hline & \multicolumn{2}{|c|}{$\begin{array}{c}\text { TCDD Low } \\
(0.0031 \mathrm{nmol} / \mathrm{g})^{1}\end{array}$} & \multicolumn{2}{|c|}{$\begin{array}{c}\text { TCDD High } \\
(0.031 \mathrm{nmol} / \mathrm{g})\end{array}$} \\
\hline & $\mathrm{SC}$ & NBH & SC & NBH \\
\hline $\begin{array}{l}\text { Bile mean } \\
{[\mathrm{TCDD}]} \\
(\mathrm{nmol} / \mathrm{g}) \\
\end{array}$ & $\begin{array}{l}0.024 \\
\pm 0.0049\end{array}$ & $\begin{array}{l}0.021 \\
\pm 0.0038\end{array}$ & $\begin{array}{l}0.12 \\
\pm 0.0079^{*}\end{array}$ & $\begin{array}{l}0.063 \\
\pm 0.0056\end{array}$ \\
\hline $\begin{array}{l}\text { Carcass mean } \\
{[\mathrm{TCDD}]} \\
(\mathrm{nmol} / \mathrm{g})\end{array}$ & $\begin{array}{l}0.00076 \\
\pm 0.000035\end{array}$ & $\begin{array}{l}0.0011 \\
\pm 0.00045\end{array}$ & $\begin{array}{l}0.0029 \\
\pm 0.00031\end{array}$ & $\begin{array}{l}0.0043 \\
\pm 0.00029\end{array}$ \\
\hline $\begin{array}{l}\text { Application Site } \\
\text { mean [TCDD] } \\
\text { (nmol/g) }\end{array}$ & $\begin{array}{l}0.0021 \\
\pm 0.00044\end{array}$ & $\begin{array}{l}0.0041 \\
\pm 0.0013\end{array}$ & $\begin{array}{l}0.0078 \\
\pm 0.00026^{*}\end{array}$ & $\begin{array}{l}0.018 \\
\pm 0.00069\end{array}$ \\
\hline $\begin{array}{l}\text { Percent } \\
\text { Recovery }\end{array}$ & $37 \pm 3.6$ & $34 \pm 7.9$ & $18 \pm 1.3$ & $22 \pm 1.9$ \\
\hline $\begin{array}{l}{[\text { Liver] / }} \\
{[\text { Carcass] }}\end{array}$ & 30.10 & 11.83 & 39.14 & 26.31 \\
\hline [Bile] / [Liver] & 1.05 & 1.60 & 1.05 & 0.57 \\
\hline Liver $\left(\mathrm{TCF}^{2}\right)$ & $\begin{array}{l}6.41 \\
\pm 1.77 \\
\end{array}$ & $\begin{array}{l}4.21 \\
\pm 0.78 \\
\end{array}$ & $\begin{array}{l}3.72 \\
\pm 0.65\end{array}$ & $\begin{array}{l}3.57 \\
\pm 1.02 \\
\end{array}$ \\
\hline Bile (TCF) & $\begin{array}{l}6.12 \\
\pm 2.02 \\
\end{array}$ & $\begin{array}{l}6.72 \\
\pm 1.23 \\
\end{array}$ & $\begin{array}{l}3.91 \\
\pm 0.26 \\
\end{array}$ & $\begin{array}{l}2.03 \\
\pm 0.18 \\
\end{array}$ \\
\hline Carcass (TCF) & $\begin{array}{l}0.20 \\
\pm 0.045 \\
\end{array}$ & $\begin{array}{l}0.36 \\
\pm 0.15 \\
\end{array}$ & $\begin{array}{l}0.095 \\
\pm 0.0099 \\
\end{array}$ & $\begin{array}{l}0.14 \\
\pm 0.0094\end{array}$ \\
\hline $\begin{array}{l}\text { Application Site } \\
\text { (TCF) }\end{array}$ & $\begin{array}{l}0.46 \\
\pm 0.089 \\
\end{array}$ & $\begin{array}{l}1.33 \\
\pm 0.42 \\
\end{array}$ & $\begin{array}{l}0.25 \\
\pm 0.0085 \\
\end{array}$ & $\begin{array}{l}0.57 \\
\pm 0.022\end{array}$ \\
\hline
\end{tabular}

${ }^{1}$ Nominal concentrations of TCDD, converted from ng/g fish, applied to fish in each treatment group. ${ }^{2} \mathrm{TCF}=$ Tissue TCDD concentration / nominal TCDD dose. ${ }^{* 3} \mathrm{H}$-TCDD concentration is significantly different $(\mathrm{p}<0.05,2$-way ANOVA comparing site and treatment) from the concentration in reference fish. 


\section{$\underline{\text { Discussion }}$}

In prior studies we have found that feral NBH Fundulus heteroclitus appear to be resistant to the induction of CYP1A1 following both environmental and laboratory exposures to AhR agonists. This resistance could be the result of a number of different possible mechanisms. In this study we attempt to differentiate between the heritable and non-heritable mechanisms. To do this we exposed lab-reared offspring of NBH fish to 3 different AhR agonists; TCDF, TCDD, and BNF, and then examined the induction of CYP1A1 as well as several other chemical and biochemical endpoints.

\section{CXP1A1}

In experiments 1 and 2 hepatic EROD activity was not significantly induced in the NBH offspring by any of the compounds used, at any dose. Mean hepatic EROD activities in experiment 1 were similar to EROD activities in field and control NBH and SC Fundulus from prior studies. These EROD activities ranged from 40 to $736 \mathrm{pmol} / \mathrm{min} / \mathrm{mg}$ (Ch. 2 \& 3). In comparison, mean EROD activity in feral SC male fish treated with 7.6 $\mathrm{nmol} / \mathrm{kg}$ TCDF was $2850 \mathrm{pmol} / \mathrm{min} / \mathrm{mg}$. Thus it appears that the resistance to CYP1A1 induction seen in feral NBH fish is maintained in lab-reared offspring of these fish.

CYP1A1 protein was also not induced by TCDF in the NBH offspring. In these fish the levels of CYP1A1 protein, regardless of treatment, were most similar to CYP1A1 protein levels in control feral NBH fish (mean $0.007 \mathrm{pmol} / \mathrm{ug}$ ). For comparison, mean CYP1A1 protein levels in SC fish treated with $7.6 \mathrm{nmol} / \mathrm{kg}$ TCDF were $0.08 \mathrm{pmol} / \mathrm{ug}$. CYP1A1 mRNA was also not induced by TCDF in the NBH offspring. Similarly, resistance to CYP1A1 induction was pre-translational in the feral NBH Fundulus (Ch. 3).

In experiment 2 , offspring from both sites were used, so CYP1A1 inducibility could be directly compared between sites. EROD activity in SC offspring was induced by both the $1 \mathrm{ng} / \mathrm{g}$ (7.5-fold) and $10 \mathrm{ng} / \mathrm{g}$ (13-fold) TCDD treatments. EROD activity was not induced in NBH offspring by either of the TCDD treatments. In this experiment, there 
was also a difference in the control EROD activities, which was not seen in any of the prior experiments. NBH offspring had significantly higher mean control EROD activity than the SC offspring. This increase in 'basal' expression of CYP1A1 is similar to results found by Prince and Cooper (Prince, 1995b) in a resistant population of Fundulus heteroclitus from Newark Bay, NJ.

As in experiments with feral NBH fish, IHC analysis of extrahepatic tissues in both experiments revealed that the lack of CYP1A1 inducibility by halogenated AhR agonists was systemic. In contrast to this result, IHC analysis of extrahepatic tissues from BNF treated NBH offspring revealed significant induction of CYP1A1 in the vascular endothelium of a number of tissues. Induction of CYP1A1 by BNF was also seen in the $\mathrm{SC}$ offspring. There was no consistent difference in the magnitude of induction between sites. These results are in sharp contrast to all of the prior results from experiments in $\mathrm{NBH}$ fish using halogenated compounds and confirm results from an in vitro experiment using primary hepatocytes from feral NBH and SC Fundulus where hepatocytes from $\mathrm{NBH}$ fish were 14-fold less sensitive to TCDD but only 3-fold less sensitive to BNF than hepatocytes from SC fish.

\section{Phase II Enzymes}

Prior GST and UGT results from experiments in feral NBH and SC Fundulus were contradictory. In experiments using TCDF, GST activity was induced only in gonadally regressed male NBH Fundulus, but not in gonadally regressed male SC or in gravid female $\mathrm{SC}$ or NBH fish. In this study GST activity was not induced in gonadally regressed NBH $\mathrm{F}_{1}$ fish. It is possible that the PCB body burdens of the non-reproductively active feral male NBH fish could have affected the induction of GST activity, perhaps by exceeding a threshold concentration of AhR agonist required before induction of GST activity can occur. The lack of induction in the NBH offspring would seem to support this hypothesis. The close agreement between GST activity in feral SC fish (mean $\sim 390 \mathrm{nmol} / \mathrm{min} / \mathrm{mg}$ ) and in NBH offspring (mean $\sim 370-380 \mathrm{nmol} / \mathrm{min} / \mathrm{mg}$ ) in comparison to feral NBH fish (mean 
$\sim 580 \mathrm{nmol} / \mathrm{min} / \mathrm{mg}$ in control and $1000 \mathrm{nmol} / \mathrm{min} / \mathrm{mg}$ in $50 \mathrm{nmol} / \mathrm{kg}$ TCDF treated fish) lends further support to this idea.

The assay used to measure GST activity in all of these studies integrates the activities of multiple GST isozymes. The activity of different GST isozymes in mammals can be modulated by thyroxin, growth hormone, epoxides, glucocorticoids, as well as HAHs and other xenobiotics (reviewed in (Hayes and Pulford, 1995)). While the reproductive status and sex of the fish were the same in the feral and offspring experiments, other factors such as age and body size differed and could be responsible for the lack of GST inducibility.

UGT activity was also not induced by TCDF in the NBH offspring. This agrees with results from studies using male and female feral NBH and SC fish. The UGT activities in NBH offspring were similar to those measured in previous experiments.

\section{Chemistry}

PCBs: NBH and SC lab-reared offspring had similar PCB concentrations and congener distributions. This suggests that PCBs deriving from maternal transfer to the egg contribute little to this adult body burden. The presence of one congener (200) that constituted a major fraction of the PCBs (15-31\%) in offspring from both sites suggested that the source of PCBs to both the NBH and $\mathrm{SC} \mathrm{F}_{1}$ fish was similar. This congener also constituted a major fraction (29\%) of the PCBs in the livers of SC fish but not in the livers of NBH fish or in the carcasses of feral fish from either site (Ch. 2). The close agreement in 138 ratios of congeners which are potential CYP1A1 substrates between $\mathrm{SC}$ and $\mathrm{NBH}$ fish suggests that there is not a significant difference in metabolic capacity between these fish.

TCDD: The similar ${ }^{3} \mathrm{H}-\mathrm{TCDD}$ distributions at the end of a 3 day exposure suggest that the uptake of TCDD is not substantially different in fish from either site. These results demonstrate that the lack of hepatic CYP1A1 inducibility is not the result of decreased liver concentrations of TCDD. Also the range of hepatic ${ }^{3} \mathrm{H}-\mathrm{TCDD}$ concentrations (from 
$\sim 5$ to $55 \mathrm{ng} / \mathrm{g}$ ) overlaps remarkably well with the range of TEQs reported in chapter 2 (from $\sim 7$ to $70 \mathrm{ng} / \mathrm{g}$ ).

\section{Mechanism of Resistance}

The results of these experiments suggest that the resistance to CYP1A1 inducibility in NBH fish is heritable. While it is possible that embryonic exposure to PCBs maternally transferred could have influenced the subsequent responsiveness of $F_{1}$ fish, results from experiments in second generation NBH embryos in which resistance persists (Nacci et al., 1999) argues against this possibility.

The difference in sensitivity to halogenated versus non-halogenated compounds in both feral and lab-reared NBH fish has also been seen in another population of fish from a PCB contaminated site. CYP1Al mRNA levels in tomcod from the Hudson River are induced by exposure to BNF but not by exposures to PCB 77 or Aroclor 1254 (Wirgin et al., 1992). Unlike killifish from NBH, CYP1Al expression in tomcod from the Hudson River is environmentally induced (Kreamer et al., 1991). Wirgin et al. suggested that this difference in sensitivity might be the result of different molecular pathways regulating the induction of CYP1Al expression. The 4S PAH binding protein has been proposed as a possible receptor for a PAH-dependent signal transduction pathway (Houser and Woodfork, 1990). The two AhRs in fish could also be involved in differential signal transduction pathways for $\mathrm{HAHs}$ and PAHs, if these receptors have different ligand binding affinities and specificities. This difference in sensitivity could also arise from an alteration in the AhR that results in a greater decrease in affinity for halogenated compounds than for non-halogenated compounds.

As in prior studies all of the CYP1A1 results suggest that the mechanism of resistance rests in the AhR signal transduction pathway. The pre-translational and systemic nature of the resistance along with the difference in sensitivity to halogenated versus nonhalogenated compounds in both feral and lab-reared NBH fish strongly indicates the involvement of the AhR pathway in this resistance. That this resistance is heritable implies 
that the change in the AhR pathway is not the down-regulation of AhR protein that is found in individual animals after long-term or repeated exposures to an $\mathrm{AhR}$ agonist (Reick et al., 1994; Roman et al., 1998).

The results of this and previous studies suggest that NBH Fundulus have developed a heritable, pre-translational, and systemic resistance to induction of CYP1A1 by halogenated AhR agonists. The development of heritable resistance to halogenated AhR agonists implies that the PCB concentrations in NBH have exerted a strong selective pressure on the population of Fundulus occurring there. The probable loss of genetic diversity that accompanied the development of this resistance may have implications for the ability of this population to tolerate other stressors. The exact nature of the mechanism of this resistance may help to predict how this population will respond to future environmental changes and is the subject of continuing studies. 
Chapter 5: HEPATIC ARYL HYDROCARBON RECEPTOR (AHR) CONTENT IN DIOXIN-RESISTANT KILLIFISH AS ASSESSED BY LIGAND BINDING ASSAYS.

Introduction

168

Materials and Methods

170

Materials

170

Fundulus Collection and Maintenance

170

Fundulus Treatments

170

Cytosol Preparation

170

Catalase Labeling

171

Sucrose Gradients

171

Photoaffinity Labeling

172

Fundulus AhR1 and AhR2 Synthesis 173

Results

174

Sucrose Gradients

174

Photoaffinity Labeling

181

Discussion 


\section{Introduction}

In previous papers, we showed that Fundulus heteroclitus (killifish) from New Bedford Harbor (NBH), MA, a federal Superfund site heavily contaminated with polychlorinated biphenyls (PCB), appeared to have developed resistance to the effects of these chemicals. This resistance was primarily characterized by a lack of cytochrome P450 1A1 (CYP1A) inducibility following either environmental or laboratory exposures to compounds that induce CYP1A1 expression in fish from a reference site (Scorton Creek, MA (SC)). This resistance was systemic, pre-translational, and heritable. These and other results suggested that the mechanism of this resistance may lie in the aryl hydrocarbon receptor $(\mathrm{AhR})$ signal transduction pathway.

The AhR signal transduction pathway mediates nearly all of the effects of planar halogenated aromatic hydrocarbons $(\mathrm{pHAH}$, which include some PCBs, dibenzo-pdioxins, and dibenzofurans) and polycyclic aromatic hydrocarbons (PAH) (Fernandez Salguero, Hilbert et al., 1996). Compounds that act through the AhR pathway first bind to the cytosolic form of the receptor. This results in the migration of the AhR into the nucleus and the release of heat shock protein 90 (HSP90). In the nucleus, the AhR dimerizes with the AhR nuclear translocator (ARNT). This complex can then bind to regulatory elements (called dioxin responsive elements, DREs) and affect the expression of downstream genes (reviewed in Hankinson 1995). In mammals, these genes include phase I xenobiotic metabolizing enzymes (i.e. CYP1A1) and phase II xenobiotic metabolizing enzymes (i.e. glutathione S-transferase-Ya and UDP-glucuronosyltransferase $1^{* 06)}$ (Nebert, Puga et al., 1993).

The AhR pathway is also present in teleosts, including killifish (Hahn, 1995) Unlike mammals, killifish and a few other teleosts have been shown to have two distinct AhRs (Hahn, Karchner et al., 1997); both of which are capable of binding TCDD and DREs. The primary form of ARNT in killifish, identified as an ARNT2 (Powell, Karchner et al., 1999), is also different than in mammals. Despite these differences, the AhR 
pathway in fish functions like the mammalian AhR pathway. DREs have been identified in the 5' flanking region of the killifish CYP1A1 gene (Powell, personal communication) and expression of this gene is clearly induced after exposure to AhR agonists. Therefore the lack of CYP1A1 inducibility in NBH fish, which could be overcome in vitro with sufficiently high concentrations of inducer (14-fold higher when 2,3,7,8tetrachlorodibenzo-p-dioxin (TCDD) was used as the inducer), suggested that the AhR pathway might be involved in the mechanism of resistance of NBH fish.

In Chapter 5, we begin to address this possibility by examining the expression of the AhR in killifish from NBH and SC. Specific binding was assessed in both field fish and fish held in the laboratory for 3 months to determine if AhR binding capacity changed over time. AhR binding was also examined in $\mathrm{NBH} \mathrm{F}_{1}$ fish to assess $\mathrm{AhR}$ expression in relatively uncontaminated fish with the NBH genetic background. 


\section{Materials and Methods}

Materials: Frozen krill was purchased from MidJersey Pet Supply (Carteret, NJ). ${ }^{3} \mathrm{H}-$ 2,3,7,8-TCDD was purchased from ChemSyn (Lenexa, KS). ${ }^{14} \mathrm{C}$-formaldehyde and ${ }^{14} \mathrm{C}$ ovalbumin were purchased from NEN (Boston, MA). ${ }^{125} \mathrm{I}-2$-azido-3-iodo-7,8dibromodibenzo- $p$-dioxin $\left({ }^{125} \mathrm{I}_{-} \mathrm{N}_{3} \mathrm{Br}_{2} \mathrm{DD}\right.$ ) was custom synthesized at NEN (Boston, $\mathrm{MA}$ ). TnT® quick coupled transcription and translation kits were purchased from Promega (Madison, WI). All other chemicals were purchased from Sigma (St Louis, MO).

F. heteroclitus collection and maintenance: Fish from NBH (6/28 - 6/29/94 7/12-7/14/95) and SC (7/18/94 7/25/96\& 7/15/97) were collected using baited minnow traps. Fish from NBH were also provided by Ken Rocha (EPA, Narragansett, RI) (9/8/97); these fish were collected using unbaited minnow traps and given to us immediately after collection. Fish were maintained in $20^{\circ} \mathrm{C}$ flowing sea water and fed Tetramin ${ }^{\circ}$ stapleflake and minced krill (NJ pet supply). In 1994 fish from both sites were bred and the resulting offspring were fed freshly hatched brine shrimp from 1 day post hatch through the end of their first year. After $\sim 6$ months the adult diet was gradually introduced and by the end of 1 year the offspring were fed the same diet as the feral adults.

Fundulus treatment: 2 year old male $\mathrm{NBH} \mathrm{F}_{1}$ fish were moved to 10 gal. experimental aquaria with $20^{\circ} \mathrm{C}$ flowing seawater 2 days prior to the start of the experiment. The fish were weighed and then dosed intraperitoneally with 50 pmol / $\mathrm{g}(\mathrm{N}=3) 2,3,7,8$ tetrachlorodibenzofuran (TCDF) dissolved in corn oil, or corn oil alone ( $N=3$, final $N=2)$. Each fish received 5 ul dosing solution per $\mathrm{g}$ of fish. After dosing, fish were held for 7 days in static seawater $\left(-20^{\circ} \mathrm{C}\right)$ during which time they were fed only Tetramin $₫$ stapleflake. After 7 days fish were killed by cervical transection. Tissues for cytosols were put immediately in ice cold AhR buffer (see below).

Cytosol preparation: Tissues were homogenized in $9 \mathrm{ml}$ of cold AhR buffer per gram of tissue. AhR buffer consists of $25 \mathrm{mM}$ MOPS ( $\mathrm{pH}$ 7.5) with $1 \mathrm{mM}$ EDTA, 5 mM EGTA, $0.02 \% \mathrm{NaN}_{3}, 20 \mathrm{mM} \mathrm{Na}_{2} \mathrm{MoO}_{4}, 10 \%$ (v:v) glycerol, $1 \mathrm{mM}$ dithiothreitol, plus protease 
inhibitors ( $20 \mathrm{uM}$ TLCK, $5 \mu \mathrm{g} / \mathrm{ml}$ leupeptin, $13 \mu \mathrm{g} / \mathrm{ml}$ aprotinin, $7 \mu \mathrm{g} / \mathrm{ml}$ pepstatin A, and 0.1 mM PMFS) (Hahn, Poland et al., 1994). Tissues were homogenized with a Teflonglass homogenizer (10 passes); intestines and gills were minced with dissecting scissors prior to homogenization. Homogenates were centrifuged for $10 \mathrm{~min}$ at $750 \mathrm{~g}$ and $10 \mathrm{~min}$ at $12,000 \mathrm{~g}$ at $4^{\circ} \mathrm{C}$. The supernatant was then centrifuged at $100,000 \mathrm{~g}$ for $70 \mathrm{~min}$ at $4^{\circ} \mathrm{C}$. This supernatant (cytosol) was removed and frozen in liquid $\mathrm{N}_{2}$.

Total protein content: Total microsomal or cytosolic protein was measured fluorometrically (Lorenzen and Kennedy, 1993).

Catalase labeling: Catalase was covalently labeled with ${ }^{14} \mathrm{C}$ by the method of DottavioMartin and Ravel (Dottavio-Martin and Ravel, 1978) as modified by Dennison (Denison, Vella et al., 1986b). Briefly, catalase (10 mg in $0.04 \mathrm{M} \mathrm{PO}_{4}$ buffer) was incubated with ${ }^{14} \mathrm{C}$-fomaldehyde (100 ul, $5.5 \mathrm{uCi}$ ) and $\mathrm{NaBH}_{3} \mathrm{CN}(2.4 \mathrm{mg})$ for $1 \mathrm{hr}$ at $25^{\circ} \mathrm{C}$. After $1 \mathrm{hr}$, the mix was diluted with $\mathrm{PO}_{4}$ buffer to $4 \mathrm{ml}$ and dialyzed for $16 \mathrm{hrs}$ at $4^{\circ} \mathrm{C}$ in a slide-alyzer (Pierce) dialysis cassette. The amount of ${ }^{14} \mathrm{C}$ incorporation was determined by counting an aliquot after dialysis.

Sucrose gradient: Sucrose gradients were run according to the method of Tsui and Okey (Tsui and Okey, 1981) with modifications. Cytosols with 1.5 to $6 \mathrm{mg} / \mathrm{ml}$ total protein were incubated for $2 \mathrm{hrs}$ at $4^{\circ} \mathrm{C}$ with $3 \mathrm{H}-\mathrm{TCDD}$ ( $2 \mathrm{nM}$ final concentration) in the presence or absence of a 100-fold excess of unlabeled TCDF. Aliquots were taken to determine total counts ( $25 \mathrm{ul})$ and for centrifugation (300 ul). Aliquots for centrifugation were layered onto linear (10 to $30 \%$ ) sucrose gradients and then centrifuged at $372,000 \mathrm{~g}$ for 130 minutes at $4^{\circ} \mathrm{C}$ with slow acceleration and deceleration in a Beckman vertical-tube rotor. Gradients were made using a Gradient master (Coombs and Watts, 1985). After centrifugation, thirty six $150 \mathrm{ul}$ fractions were collected and radioactivity was measured in a Beckman 5000TD scintillation spectrometer. ${ }^{14} \mathrm{C}$ - Ovalbumin $(3.6 \mathrm{~S})$ and ${ }^{14} \mathrm{C}$-catalase or unlabeled catalase $(11.3 \mathrm{~S})$ were added to each gradient and used as internal sedimentation markers. Specific binding was defined as the total binding (without excess unlabeled TCDF) minus non- 
specific binding (with excess unlabeled TCDF). Total counts ( $25 \mathrm{ul}$ aliquots) were used to confirm the ${ }^{3} \mathrm{H}-\mathrm{TCDD}$ concentration in each incubation.

Several modifications of the protocol were tried. Incubation of NBH cytosols with dextran-coated charcoal prior to ${ }^{3} \mathrm{H}-\mathrm{TCDD}$ addition, to potentially decrease the concentration of unbound AhR ligands present, had no effect $(N=3)$. Incubation of $S C$ cytosols $(N=1)$ with dextran-coated charcoal after ${ }^{3} \mathrm{H}-\mathrm{TCDD}$ incubations decreased specific binding; this has been reported before for teleost AhRs (Lorenzen and Okey, 1990). One hour incubations, as opposed to two hours, increased specific binding in $\mathrm{SC}$ and $\mathrm{NBH}$ cytosols ( $\mathrm{N}=1$ per site). Three attempts were made to determine ligand binding characteristics using a range of ${ }^{3} \mathrm{H}$ TCDD concentrations $(0.4,0.6,1.0,1.8,3.4,6.5,12.3 \mathrm{nM})$. No specific binding was seen at any concentration in these experiments. In one experiment, the lack of specific binding may have been due to problems with the $\mathrm{pH}$ of the buffers used. The reasons for the failure of the other two experiments are unknown.

Catalase assay: Aliquots ( $5 \mathrm{ul}$ ) of each fraction were incubated with TEB (0.05 M Tris$\mathrm{HCl}, 0.25 \mathrm{mM}$ EDTA, $1 \mathrm{mM} \mathrm{H}_{2} \mathrm{O}_{2}$ ). The absorbance at $240 \mathrm{~nm}$ was read for $1 \mathrm{~min}$. The catalase peak was defined as the fraction(s) with the maximal change in absorbance per min divided by the sample volume $(\mathrm{ml})$, the extinction coefficient and the protein concentration.

Phottoaffinity labeling: The method of Poland et al. (Poland, Glover et al., 1986) was followed with modifications. Cytosols $(1 \mathrm{mg} / \mathrm{ml}, 250 \mathrm{ul})$ were incubated with ${ }^{125} \mathrm{I}-$ $\mathrm{N}_{3} \mathrm{Br}_{2} \mathrm{DD}(1.0 \mathrm{nM})$ in the presence or absence of 100 or 200 -fold excess unlabeled TCDF for $2 \mathrm{hrs}$ at $4^{\circ} \mathrm{C}$. All steps were performed under red-light until after the UV exposure. After $2 \mathrm{hrs}$, samples were exposed to UV light (two 320 watt bulbs, Sylvania) at $4 \mathrm{~cm}$ for $2 \mathrm{~min}$ at $\mathrm{rt}$. Immediately after the UV exposure, soybean trypsin inhibitor (100 ug, $50 \mathrm{ul}$ ) and cold acetone $(1 \mathrm{ml})$ were added. The samples were then transferred to $1.5 \mathrm{ml}$ eppendorf tubes and incubated overnight at $-20^{\circ} \mathrm{C}$. The samples were then centrifuged at $4000 \mathrm{~g}$ (or $3000 \mathrm{~g}$ ). This pellet was washed with cold acetone : water $(9: 1)$ and centrifuged again. This pellet was dissolved in $100 \mathrm{ul}$ of sample treatment buffer $(0.063 \mathrm{M}$ 
Tris $\mathrm{HCl}, 10 \%$ glycerol, $1 \% \mathrm{SDS}, 0.002 \%$ bromophenol blue, $5 \% \beta$-mercaptoethanol) and loaded onto a 7.5\% SDS-PAGE gel. After electrophoresis, the gel was stained with coumassie blue and dried. The gels were exposed to X-ray film (Kodak Biomax or AR film) for 4 to 14 days (Biomax) or $45+$ days (AR film). Band intensity was measured as integrated densities. Specific binding is defined as in the sucrose gradient method. Several potential positive controls were analyzed with each set Fundulus samples. These included cytosols from Hepa-1 cells, which over express AhR (Hankinson, 1983), and in vitro synthesized (see below) Fundulus AhR1 and AhR2. Specific binding for each sample was normalized to the intensity of Hepa-1 total binding measured on the same film.

Fundulus AhR1 and AhR2: For each photoaffinity gel Fundulus AhR1 and AhR2 were synthesized in vitro using the TnT® quick coupled transcription and translation (Promega, Madison, WI) method. Briefly, cDNA was incubated for $90 \mathrm{~min}$ at $30^{\circ} \mathrm{C}$ with the master mix (which contained rabbit reticulocyte lysate) and methionine (1 mM). After $90 \mathrm{~min}$, this was transferred to ice. Either a full (AhR2) or half (AhR1)-reaction was diluted with AhR buffer to $250 \mathrm{ul}$ and used for each photoaffinity incubation. 


\section{$\underline{\text { Results }}$}

We have previously reported that killifish from NBH are resistant to CYP1A1 induction following either environmental or laboratory exposures to AhR agonists. We have hypothesized that this heritable resistance is the result of an alteration in the AhR pathway in these fish. In these studies hepatic AhR content was assessed by ligand binding in feral Fundulus from SC and NBH and control and in TCDF-treated 2-year-old NBH $F_{1}$ fish.

\section{Sucrose Gradients:}

Specific binding could be detected in some cytosols from both $\mathrm{SC}$ and $\mathrm{NBH}$ fish (Fig 5-1 \& 2). Overall, more cytosols from SC fish than from NBH fish had at least some detectable specific binding (Table 1). The mean specific binding in cytosols from SC fish was higher than that in cytosols from NBH fish $(p=0.058)$. When hepatic cytosols from SC and NBH fish that had been held for over 12 months were compared this difference in specific binding did not appear to persist. Several of the cytosols also had a large nonspecific peak that sedimented in the same range as the AhR (Fig. 5-2). This peak corresponded to the presence of a large protein band $(>200 \mathrm{kD})$ in coumassie stained gradient SDS-page gels (Fig 5-3). 
Table 1:AhR Specific Binding in Fundulus heteroclitus from SC and NBH

\begin{tabular}{|l|c|c|}
\hline Time Since Collection & SC & NBH \\
\hline 1 Day & $\begin{array}{c}10 \pm 5 \\
(0-35 ; n=7)\end{array}$ & $\begin{array}{c}2 \pm 0.2 \\
(0-3 ; n=7)\end{array}$ \\
\hline $\begin{array}{l}\text { \# of samples with detectable } \\
\text { specific binding }\end{array}$ & 5 out of 7 & 2 out of 7 \\
\hline 90 days & $\begin{array}{c}4 \\
(2-5 ; n=2)\end{array}$ & nd \\
\hline 1 Year+ & $\begin{array}{c}5 \\
(0-9 ; n=2)\end{array}$ & $\begin{array}{c}4 \\
(0-15 ; n=4)\end{array}$ \\
\hline $\begin{array}{l}\text { \# of samples with detectable } \\
\text { specific binding }\end{array}$ & 1 out of 2 & 1 out of 4 \\
\hline
\end{tabular}

Values are $\mathrm{fmol}$ TCDD bound $/ \mathrm{mg}$ total cytosolic protein added. Mean \pm standard error (range, number of individuals assayed). nd = no samples analyzed. Samples with no detectable specific binding were defined as $\sim 2 \mathrm{fmol} /$ $\mathrm{mg}$ for the determination of means and standard errors. The limit of detection was $\sim 2 \mathrm{fmol} / \mathrm{mg}$. 
Fig. 5-1: AhR binding in hepatic cytosols from SC fish. The solid curve is total binding and the dashed curve is non-specific binding. The ${ }^{14} \mathrm{C}$ ovalbumin peak is marked on each graph. H97-059 is a male fish and displays a distinct specific binding peak between fractions 13 and 18. H97-060 is a female fish with a small non specific peak between fractions 14 and 19. 

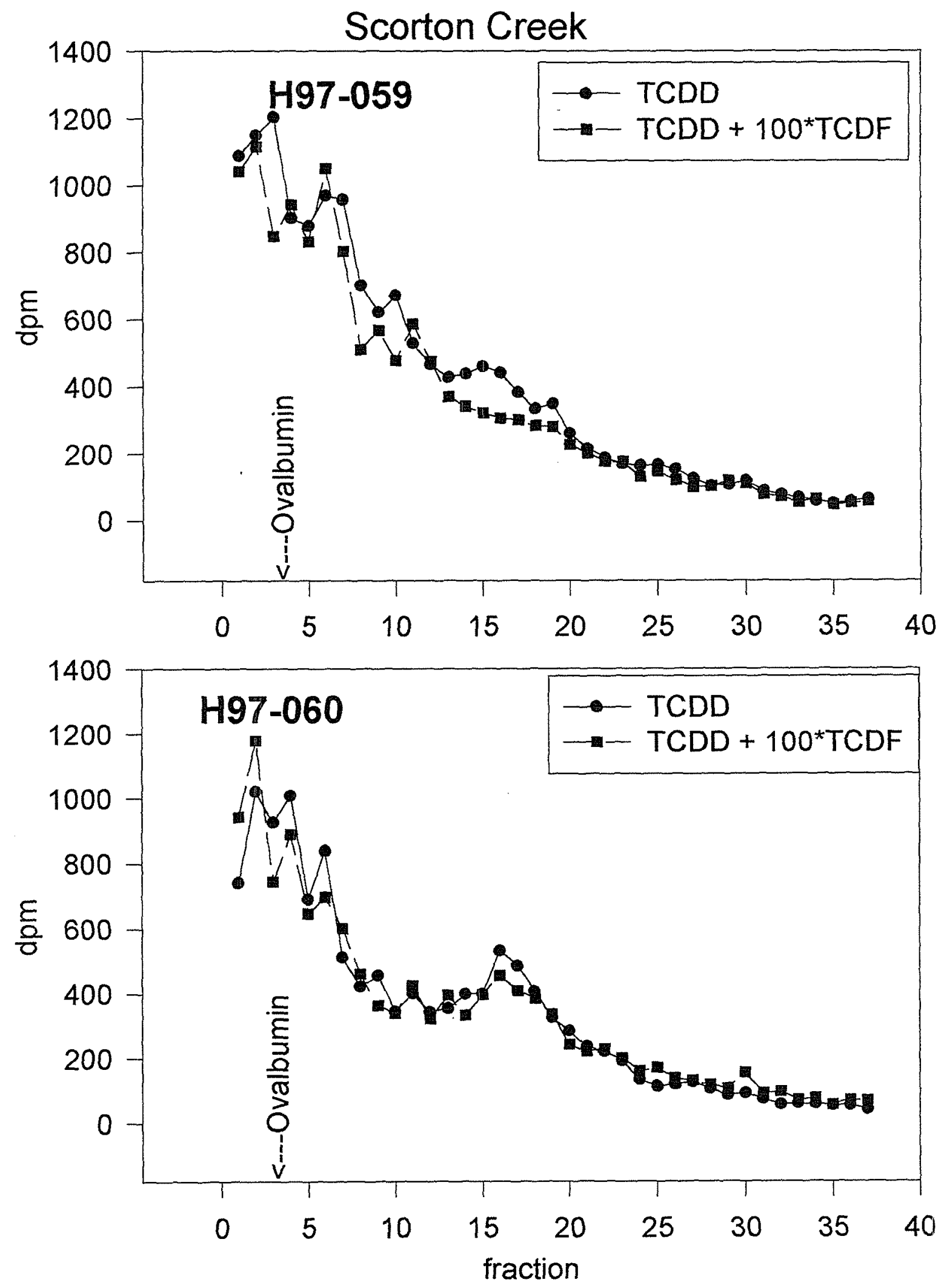
Fig. 5-2: AhR binding in hepatic cytosols from NBH fish. The solid curve is total binding and the dashed curve is non-specific binding. The ${ }^{14} \mathrm{C}$ ovalbumin and catalase peaks are marked on each graph. Sedimentation coefficients for specific and non-specific peaks were determined relative to these markers. H97-100 is a male fish that displays a distinct non-specific binding peak of 10-11S. H97-101 is a male fish with a slight specific peak of $\sim 10-11 \mathrm{~S}$. 


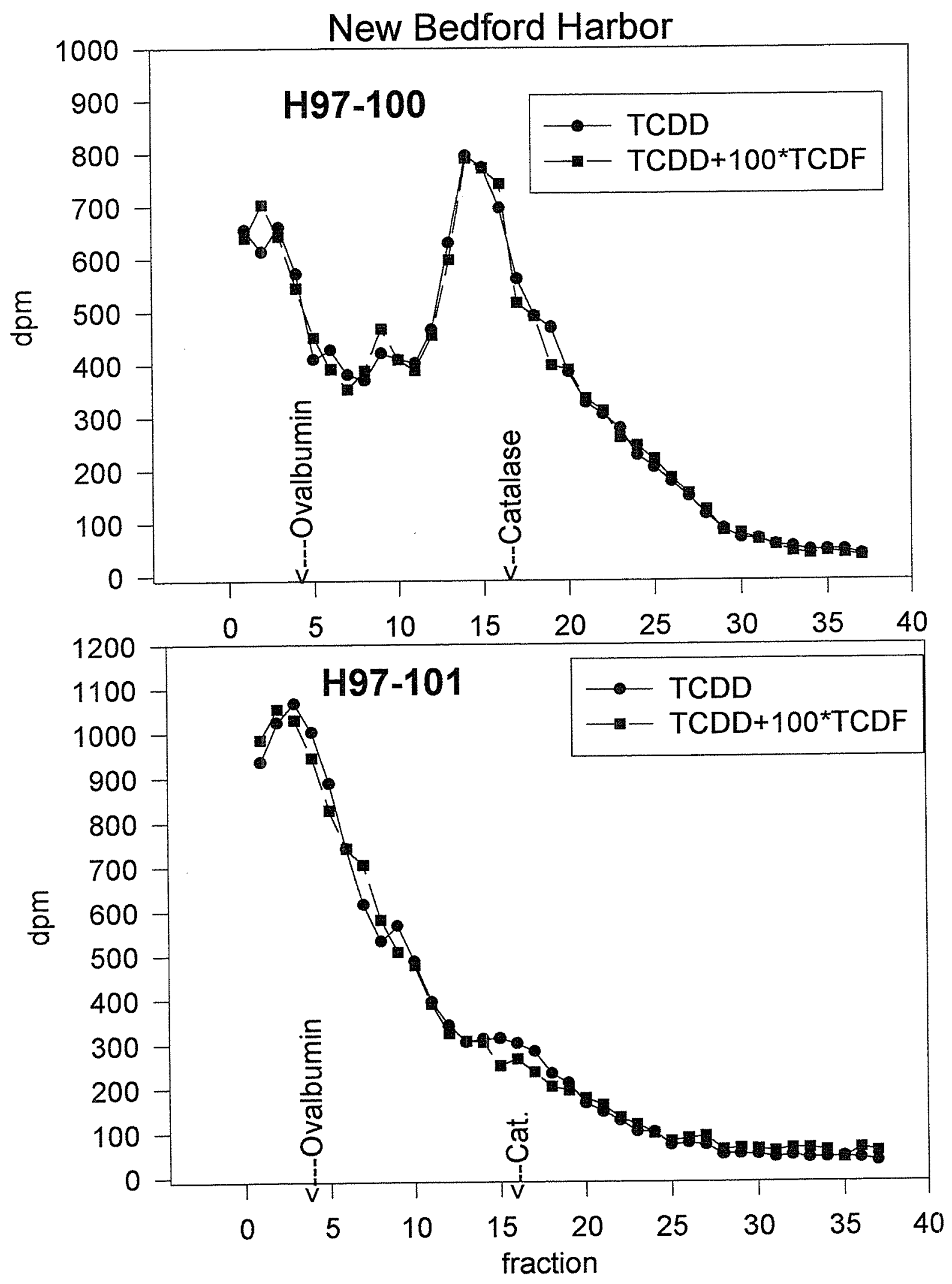




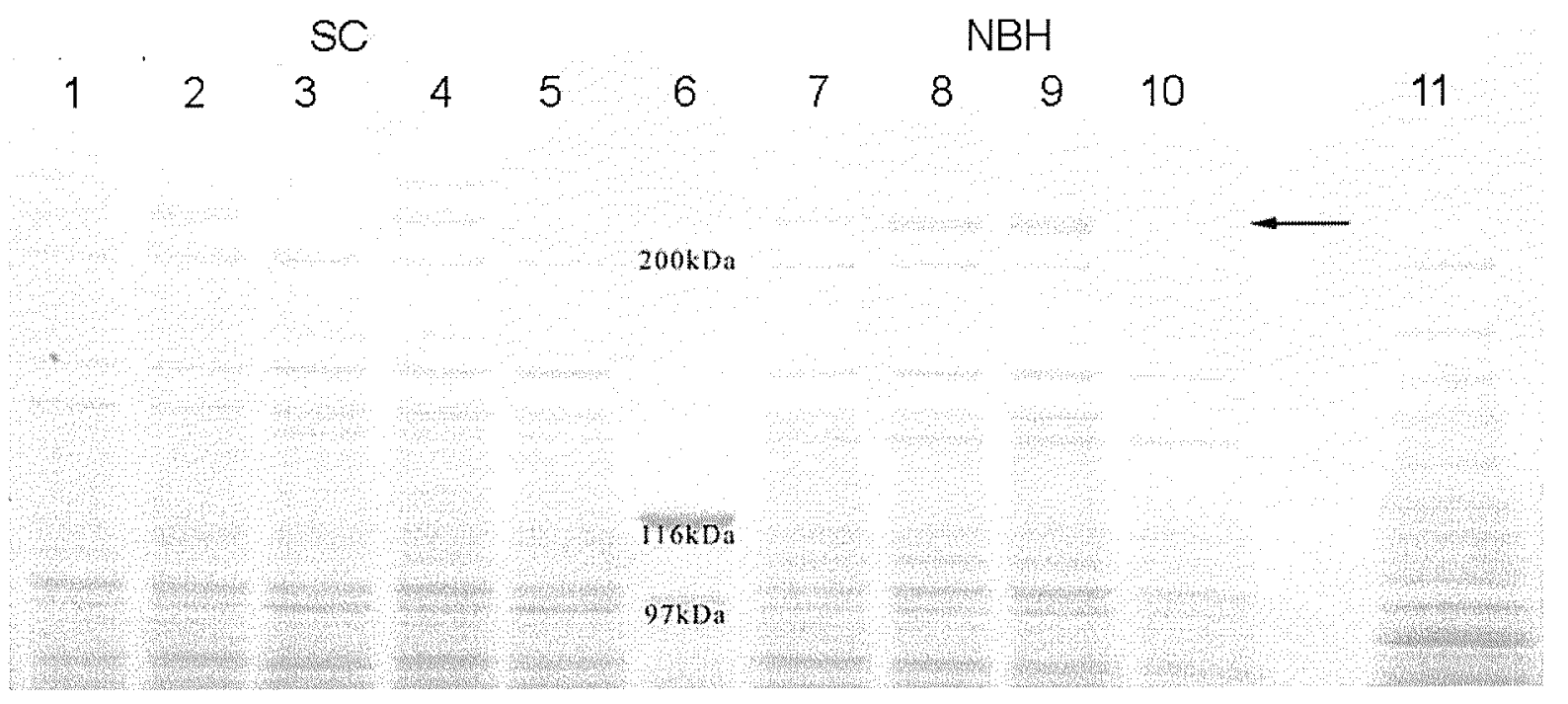

Fig. 5-3: Protein bands stained with coumassie blue from SC and NBH cytosols. The arrow indicates a band of $>200 \mathrm{kD}$ that occurs only in those cytosols which also had non-specific peaks when analyzed by sucrose gradient centrifugation. Lanes 1-5 have SC cytosols; lane 6 has molecular weight markers; lanes 7 - 10 are NBH cytosols, lane 11 has Hepa-1 cytosol. Cytosols in lanes 2, 4, and 7 - 9 had non-specific peaks. 


\section{Photoaffinity Labeling:}

In the sucrose binding assay the ligand is reversibly bound to the receptor. Ligand dissociation during the course of the assay may result in an underestimate of the total specific binding present. The photoaffinity method overcomes this potential difficulty through formation of a covalently linked ligand-receptor complex. This complex can be visualized on SDS-page gels. This method also allowed for separation of the non-specific peak found in many of the sucrose gradient samples.

As with the results of the sucrose gradient assays, cytosols from $\mathrm{SC}$ fish had significantly higher specific binding than cytosols from NBH fish (Fig. 5-4 \& 5). There was also significantly more specific binding in female fish from either site than in male fish from the same site. The majority of both the sucrose gradient assays and the photoaffinity assays had been done using cytosols prepared from fish one day after collection. To determine if the differences in specific binding persisted, cytosols were prepared from SC and NBH fish 90 days after collection (Fig. 5-5). Interestingly the sex difference persisted but the site difference did not.

The PCB body burdens in the NBH fish could potentially affect the results of these AhR assays in a number of ways. PCBs present in the hepatic may bind to the AhRs preventing the radio-labeled ligand from binding. This could result in underestimates of AhR content. The PCBs may also effect the AhR protein content. Down-regulation of AhR protein has been reported in rodents after exposures to AhR ligands (Reick, Robertson et al., 1994). Again, this could result in underestimates of AhR content. Finally, PCBs may induce AhR expression (Landers, Winhall et al., 1991), resulting in an overestimate of $\mathrm{AhR}$ content.

To determine what effect the high PCB body burdens might have on AhR expression in NBH fish, AhR binding was examined in NBH $F_{1}$ fish. There was little or no AhR binding in cytosols prepared from control $\mathrm{NBH} \mathrm{F}_{1}$ fish (Fig 5-5), similar to results seen in male NBH fish 1 day after collection. AhR binding was higher in cytosols of NBH $\mathrm{F}_{1}$ fish that had been treated with $2,3,7,8-\mathrm{TCDF}$ than in untreated $N B H F_{1}$ fish. 
Three potential positive controls were analyzed with each set of Fundulus cytosols, Hepa-1 cytosols and in vitro synthesized Fundulus AhR1 and AhR2. Analysis of the two Fundulus AhRs also had the potential to allow for discrimination between AhR1 and AhR2 content in the Fundulus cytosols. Hepa-1 cytosols gave consistent bands that were readily quantified on every gel. AhR1 also had measurable specific binding on all of the films. Binding to AhR2 was undetectable, except in one experiment where a very faint band was observed. The specific bands in nearly all of the cytosolic samples appeared to be smaller than AhR1 (Fig 5-4), as was the one AhR2 specific band.

There was a non-specific band of large molecular weight present in a number of the cytosols from SC and NBH feral fish (Fig. 5-5). A similar band was reported by Hahn et al. (Hahn, Poland et al., 1994) as being specific to female fish and putatively identified as vitellogenin. Interestingly, while this band is specific to females in SC fish, it occurs in both male and female NBH fish. Furthermore, it is not present in the NBH $F_{1}$ fish (all males). This suggests that some of the compounds in NBH may be having estrogenic effects on the NBH killifish. 


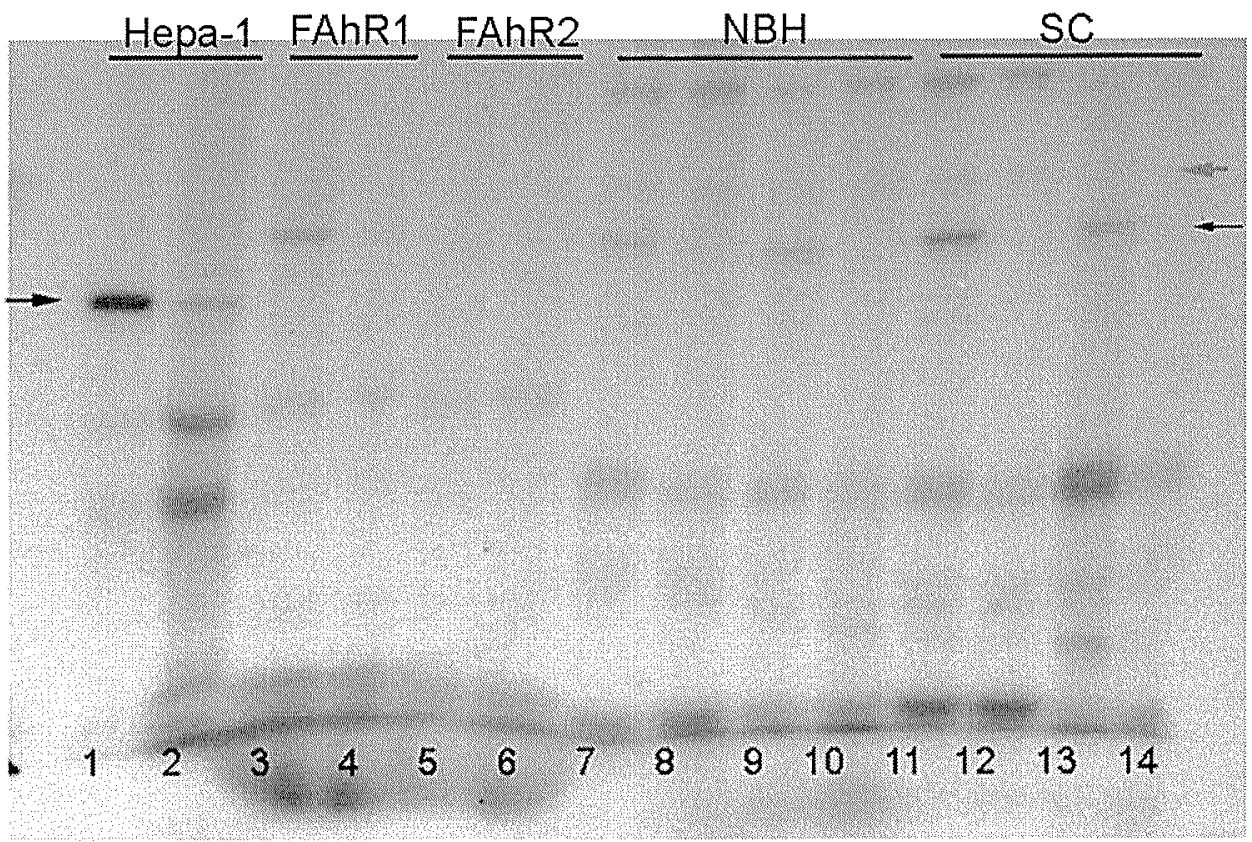

Fig 5-4: Representative photoaffinity gel with hepatic cytosols from female SC and NBH Fundulus one day after collection. The black arrows indicate the putative AhR bands. The gray arrow indicates the non-specific bands. Odd lanes: ${ }^{125} \mathrm{I}_{-} \mathrm{N}_{3} \mathrm{Br}_{2} \mathrm{DD}$ alone measuring total binding. Even lanes: ${ }^{125} \mathrm{I}_{-} \mathrm{N}_{3} \mathrm{Br}_{2} \mathrm{DD}+100$-fold excess $\mathrm{TCDF}$, measuring non-specific binding. Lanes 1-2: Hepa-1 cytosol, lanes 3-4: Fundulus AhR1, lanes 5-6: Fundulus AhR2, lanes 7-10: hepatic cytosols from NBH Fundulus, lanes 11-14: cytosols from SC $\underline{\text { Fundulus }}$ The dried gel was exposed to Kodak Biomax film for 3 days. 
Fig. 5-5: Photoaffinity analysis of AhR binding in SC and NBH feral fish 1 and 90 days after collection and in control and TCDF treated $\mathrm{NBH} \mathrm{F}_{1}$ fish. Values were determined by measuring the integrated density of each band, calculating the specific binding by subtracting the non-specific binding from the total binding, and normalizing this value to the integrated density of the Hepa-1 total binding band. To check that the Hepa-1 band in each gel was within the linear range specific binding of each sample was also normalized to FAhR1 binding. These two methods resulted in the same pattern of differences between the samples. AhR binding was significantly higher in day $1 \mathrm{SC}$ fish than in day $1 \mathrm{NBH}$ fish and in female fish than in male fish ( $p<0.05$, 2- way ANOVA comparing site and sex). $N=4$ for each group except $\mathrm{N}=2$ for control and $\mathrm{TCDF}$ treated $\mathrm{NBH}_{1}$ fish. 


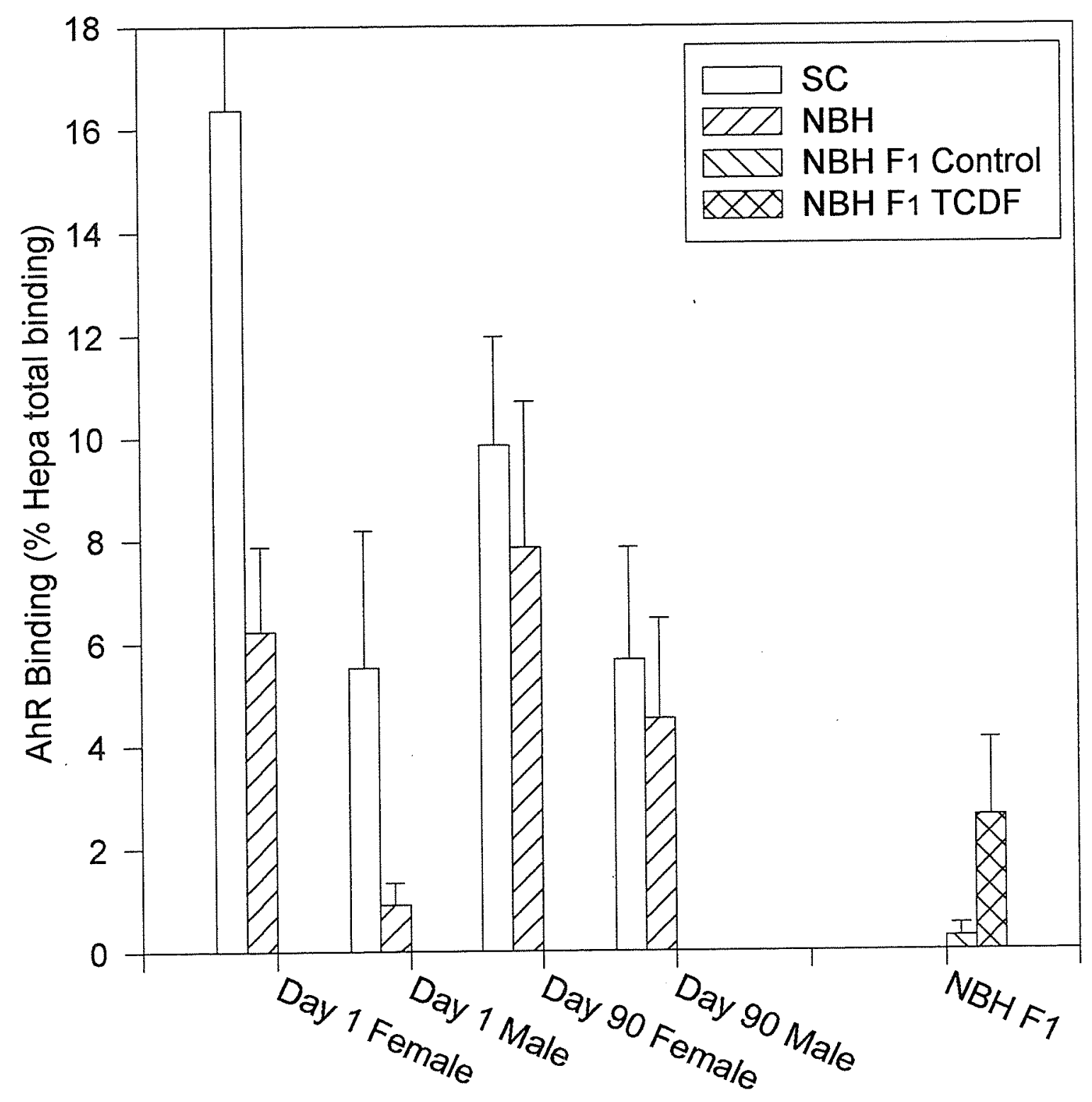




\section{Discussion}

Killifish from NBH have been shown to be resistant to some of the effects of HAH. This resistance has been characterized as a systemic, pre-translational lack of CYP1Al inducibility that is heritable. We have hypothesized that an alteration in the AhR signal transduction pathway is responsible for this resistance. In this paper AhR binding was measured in hepatic cytosols from feral $\mathrm{SC}$ and $\mathrm{NBH}$ fish as well as from $\mathrm{NBH}_{1}$ fish. In fish fresh from the field, AhR binding was higher in $\mathrm{SC}$ fish than $\mathrm{NBH}$ fish. Specific binding was also greater in male fish than in female fish (both sites). The difference in AhR binding between sites was not present in fish that had been held for 90 days. AhR binding was lowest in male NBH fish 1 day after collection and in $\mathrm{NBH}_{1}$ fish.

\section{AhR Binding}

As measured here, AhR binding is influenced by differences in ligand affinity and the cytosolic concentration of unbound AhR protein. These differences could be the result of differences in exposure history and / or genetic differences. Differences in ligand affinity could be the result of genetic differences in the AhR (Okey, Vella et al., 1989) or from differences in the ratio of $A h R 1$ to $A h R 2$, if these receptors have different ligand affinities. The ratio of $A h R 1$ to $A h R 2$ could be changed if exposure to AhR agonists has different effects on each receptor or by genetic differences in expression of either or both receptors. Differences in exposure history could result in differences in the expression of AhR1 and / or AhR2. Exposure to AhR agonists can induce (Landers, Winhall et al., 1991; Abnet, Tanguay et al., 1999) or down-regulate (Pollenz, 1996; Giannone, Li et al., 1998) AhR protein. Genetic differences can also result in differences in AhR expression (Hankinson, 1983). Finally, the higher PCB concentrations in the livers of $\mathrm{NBH}$ fish compared to SC fish (Ch. 2) could result in a depletion of free $A h R$ in the liver cytosols.

The data presented here suggest that more than one of the mechanisms above may be influencing the differences seen. The increase AhR binding in male NBH fish but not in 
male SC fish comparing cytosols from fish one day or 90 days after collection suggests that the pool of unbound AhR may be depleted in the NBH fish initially. This is supported by the presence of measurable dioxin equivalents in liver extracts of NBH fish but not in the liver extracts of SC fish ( $\mathrm{Ch} 2$ ). The free concentration of these inducing compound might decrease over time; allowing the unbound pool of cytosolic AhR to increase. But there is no such change in the female NBH fish over the same span of time.

If the low levels of AhR binding in day one NBH male fish was solely the result of depletion the available pool of unbound $\mathrm{AhR}$, then male $\mathrm{NBH} \mathrm{F}_{1}$ fish would be expected to have higher levels of AhR binding. However, this was not the case. AhR binding content in cytosols from untreated $\mathrm{NBH} \mathrm{F}_{1}$ fish was most similar to the levels seen in day one NBH male fish. AhR binding in the $\mathrm{NBH} \mathrm{F}_{1}$ fish was induced by treatment with TCDF. This suggests that in the feral fish both depletion of unbound AhR and induction of either one or both AhRs is occurring simultaneously. Induction of at least one of the AhRs is supported by differences in the mRNA expression of AhR1 between day one $\mathrm{SC}$ and NBH fish. The expression of AhR1 is ordinarily tissue specific with low expression in the liver (Karchner, Powell et al., 1999), but in feral NBH fish the expression of AhR1 mRNA is elevated in the liver and other tissues (Powell, personal communication).

There is an apparent decrease in AhR binding in hepatic cytosols from female SC fish between one day and 90 days after collection. The most significant change in the condition of these fish over the same span of time was the transition from mature to regressed gonads (Ch. 2). The changes in hormone expression over this time period may have affected the expression of $A h R$ in these fish. It is therefore interesting to note that while the female NBH fish were also undergoing the same change in gonadal status, there was not a decrease in AhR binding in these fish.

There only appeared to be one band present in each of the cytosols assayed. Analysis of hepatic AhR mRNA expression indicates that AhRl is poorly expressed while $A h R 2$ is more abundantly expressed. The position of this band relative to AhR1 varied. There was no apparent site or sex specific pattern to this variation. In the one experiment 
where a faint $\mathrm{AhR} 2$ band was visible, some of the cytosolic bands appeared to be fall between $\mathrm{AhR} 1$ and $\mathrm{AhR} 2$. This prevents any comparison of $\mathrm{AhR} 1$ and / or AhR2 content between or within sites.

\section{Mechanism of Resistance}

The differences in AhR binding between feral $\mathrm{SC}$ and $\mathrm{NBH}$ fish and $\mathrm{NBH} \mathrm{F}_{1}$ fish suggest that a decrease in the affinity of AhR for halogenated compounds and / or a change in AhR expression may be responsible for the resistance to CYP1Al inducibility in NBH fish. The difference in sensitivity to halogenated versus non-halogenated compounds in both $\mathrm{NBH} \mathrm{F}_{1}$ fish and cultured hepatocytes from NBH fish (Ch. 3 and 4) seems to support an alteration in ligand binding affinity. However, if the two different forms of AhR have different affinities for halogenated and non-halogenated compounds, than these results could also be consistent with a decrease in the expression of the form with greater affinity for halogenated compounds. Another possibility is that one of the AhRs is a repressor and has been altered so that it either has greater affinity for or is more easily induced by halogenated then non-halogenated AhR agonists and / or is more easily induced in NBH than in SC fish.

The results of previous studies suggest that Fundulus heteroclitus from NBH have developed resistance to induction of CYP1A1 by AhR agonists. This resistance is heritable, systemic, pre-translational and more pronounced for halogenated than nonhalogenated AhR agonists. The results of this study suggest that an alteration in the AhR itself is, at least in part, responsible for this resistance. Further studies are necessary to confirm this result, especially in the extrahepatic organs and in NBH and $\mathrm{SC} \mathrm{F}_{1}$ fish. 
Chapter 6: SUMMARY, CONCLUSIONS, AND SPECULATIONS

Resistance to HAH in Fundulus heteroclitus from NBH 190

Future Directions 193

Speculations on Ramifications 194 


\section{Summary and Conclusions}

The effects of multi-generational exposures to toxic compounds are only poorly understood. Few 'chronic' exposure studies consider more than one or two generations. One way to begin to examine the impact of truly multi-generational exposures is to compare the responsiveness of populations from impacted and non-impacted sites, where the impacted site is known to have been polluted for much longer than the generation time of the species being compared. In this work a population of Fundulus heteroclitus from NBH (contaminated with HAHs) was compared to a population from a reference site (SC). Chemical analysis of fish from SC indicated that fish from this site had detectable but very low concentrations of PCBs (Ch.2).

Ordinarily when killifish are exposed to HAHs expression of CYP1A1 is induced. NBH killifish had hepatic CYP1A1 expression, as measured by EROD activity, CYP1A1 protein concentration and mRNA levels, equal to or less than that in SC fish (Ch. 2). The lack of CYP1A1 induction was systemic, with the sole exception of the proximal tubules of the kidney. A number of different mechanisms could all result in the observed lack of CYP1A1 induction. These include:

1. NBH fish are so stressed by the environmental conditions that they are incapable of inducing expression of any proteins.

2. CYP1A1 activity is inhibited by the high PCB concentrations present.

3. CYP1Al protein is inactivated by the high $\mathrm{PCB}$ concentrations present.

4. CYP1Al mRNA transcription is suppressed by the high $\mathrm{PCB}$ concentrations present.

5. A heritable, $\mathrm{PCB}$ independent alteration in the AhR pathway. The higher GST and UGT activity in NBH fish compared to SC fish collected in 1997 (Ch. 2) and the significant differences in activity between tissues in the NBH fish suggests that the first possibility is not responsible for the lack of CYP1A1 induction. 
Mechanisms 2 and 3 depend on high concentrations of PCBs interacting with the CYP1A1 protein; therefore if the concentration at the site of action decreased over time CYP1A1 activity and/or protein expression may increase. The slight increase in EROD activity without any corresponding change in CYP1A1 protein during the first 3 months after collection suggests that some inhibition of EROD activity may be occurring. But this inhibition and inactivation alone can not completely explain the results in Chapter 2 . The mechanisms of inhibition of CYP1A1 activity and inactivation of CYP1A1 protein are dependent on high concentrations of HAHs (White et al., 1997). A corollary to this is that inhibition should not be overcome by exposure to additional HAHs. The induction of EROD activity in primary cultures of hepatocytes from NBH fish exposed to TCDD (Ch. 3). suggests that the inhibition of CYP1A1 activity and inactivation of CYP1A1 protein are not primarily responsible for the lack of CYP1Al inducibility in NBH fish.

Suppression of CYP1A1 mRNA might not decrease even if PCB concentrations do decrease. Down-regulation of the AhR pathway (Reick et al., 1994; Roman et al., 1998), the most probable cause of CYP1A1 mRNA suppression, may continue even after PCB concentrations have decreased. Therefore, differentiating between suppression of CYP1A1 mRNA expression and heritable resistance required experiments in lab-reared offspring of SC and NBH fish. Exposure of NBH F1 fish to TCDF or TCDD did not result in significant induction CYP1Al by any measure used (Ch. 4). These results strongly suggest that the $\mathrm{NBH}$ fish have developed a heritable form of resistance as opposed to any of the other mechanisms proposed ( 1 through 4 , above).

Heritable resistance could involve any of the steps in the AhR pathway. The most likely mechanisms would involve a change in:

1. AhR
A. Ligand binding affinity
B. Expression
C. Ratio of AhR1 to AhR2
D. DRE binding affinity 

E. ARNT binding affinity
F. Hsp90 binding affinity
G. Loss or decrease of transactivation activity

2. CYP1A1
A. Substrate binding affinity
B. Substrate turnover
C. DRE (mutation of 1 or more DREs to decrease AhR-ARNT binding

affinity)

\section{ARNT}
A. AhR binding affinity
B. DRE binding affinity
C. Loss or decrease of transactivation activity

Changes in each of these proteins have been described in resistant cultures of mouse hepatoma cells (reviewed in (Swanson and Bradfield, 1993)). While it is not yet possible to single out the exact mechanism, the results are more suggestive of a mechanism that involves a change in the AhR than the other possibilities. The general agreement between the various measures of CYP1Al expression in NBH fish (Ch. 2, 3, \& 4) and in maximal EROD activity induced in hepatocytes from $\mathrm{SC}$ and NBH fish (Ch. 3) suggests that an alteration in the coding region of the CYP1Al gene has not occurred. The exclusion of any change in CYP1A1 is supported by the difference in inducibility of GST between male $\mathrm{NBH}$ and SC fish (Ch. 3). It is extremely unlikely that a change in the promoter region of CYP1A1 would affect the induciblity of GST.

None of these results specifically suggest that there is not a change in ARNT, but the difference in sensitivity to halogenated versus non-halogenated inducers (Ch. $3 \& 4$ ) is more suggestive of a change in the AhR than in ARNT. Hepatocytes form NBH fish and NBH $F_{1}$ fish were both more sensitive to induction by a non-halogenated inducer (BNF) than by halogenated inducers (TCDD and TCDF). Only the AhR interacts directly with ligand; therefore differences in inducibilty by halogenated and non-halogenated inducers 
are far more likely to be the result of a change in AhR. This change could be in ligand binding affinities or in the expression of $\mathrm{AhR} 1$ or $\mathrm{AhR} 2$, if the two receptors have different ligand binding affinities.

Initial experiments to characterize AhR binding in the NBH fish suggest that the expression and / or binding affinity of the AhR in NBH fish is different from that in SC fish (Ch. 5). NBH fish initially had less AhR binding in hepatic cytosols, but this difference was essentially lost by 90 days post collection. Oddly, AhR binding was lowest in untreated NBH $F_{1}$ fish and was induced by TCDF treatment in these fish.

\section{Future Directions}

Overall, the data presented in this thesis characterizes a pre-translational, systemic, heritable resistance to $\mathrm{HAH}$ in a population of Fundulus heteroclitus that has been exposed to high concentrations of PCBs for multiple generations. Future research should concentrate identifying the exact mechanism of resistance in NBH fish. Differences in the expression and / or binding affinity of AhR1 and / or AhR2 seem to be the most promising avenues to explore. The establishment and maintenance of a laboratory culture of NBH killifish is essential to this research, as it would eliminate complications arising from different $\mathrm{HAH}$ body burdens.

This population could then be used to explore differences in mammalian and teleost AhR pathways. Such exploration may help to define an endogenous ligand and function of the AhR pathway. A better understanding of the teleost AhR pathway may also lead to more accurate prediction of the effects of AhR ligands in fish.

This work has focused on the dioxin-like, planar and coplanar PCBs, but most PCBs are not included in this group. This focus was deliberate; the dioxin-like PCBs are generally more toxic to organisms (reviewed in (Safe, 1993)) and exert their toxic effects primarily through a known mechanism, the AhR pathway. This does not mean that the 
non-planar congeners are not toxic and it is possible that Fundulus from NBH could have developed resistance to these compounds as well.

\section{Speculation on Possible Ramifications}

The adaptation of a population to existence in a highly polluted environment may have a number of repercussions. The strong selective pressure that this adaptation suggests will almost certainly result in a loss of genetic diversity in the affected population (reviewed in (Fox, 1995)). The consequences of such a loss of diversity depend to some extent on the rate of gene flow between populations, but will probably leave the population less able to adapt to additional stressors. The development of resistance may also complicate remediation efforts. Biomonitoring the effects of remediation could be more difficult as the response of the populations will be harder to predict. The resistance mechanism may or may not have negative consequences in a cleaner environment. Studies in AhR knock-out mice suggest that loss of the AhR can impair the immune system (Fernandez Salguero et al., 1995). Anecdotal reports and personal observations of an increased incidence of infections in NBH killifish compared to reference fish when both are held in the laboratory suggest that removal of the PCBs may negatively impact the NBH killifish population. This would be possible if the infectious organisms were more sensitive to HAHs than the NBH killifish. Also the choice of the biomarker(s) to monitor will be difficult and require characterization in fish from the resistant population.

Acquired resistance may also complicate biomonitoring efforts in general, especially when high levels of persistent pollutants are involved. When monitoring a site known to be heavily contaminated, a decrease in the response could now be interpreted either as a decrease in contamination of the site or a decrease in the sensitivity of the population to the contaminant. 
In conclusion, the data reported in this thesis describe a systemic, pre-translational, heritable resistance to HAHs in a population of killifish from a marine Superfund site. This resistance is not present in a population of killifish from a reference site with low concentrations of HAHs. The existence of populations of organisms with acquired resistance to anthropogenic compounds provides dramatic evidence of the long-term population level effects of pollution. These populations caution against the use of biomarkers without an understanding of how the processes that result in the response being measured can be altered. Finally, study of these populations represents an opportunity to expand our understanding of how toxicants exert their effects in nonmammalian organisms. 


\section{Biblography}

Able, K.W. (1984). "Variation in spawning site selection of the mummichog, Fundulus heteroclitus." Copeia 2: 522-525.

Abnet, C.C., R.L. Tanguay, M.E. Hahn, W. Heideman and R.E. Peterson (1999). "Two forms of aryl hydrocarbon receptor type 2 in rainbow trout (Oncorhynchus mykiss): Evidence for differential expression and enhancer specificity." J. Biol. Chem. 274: 15159-15166.

Andersson, T., M. Pesonen and C. Johansson (1985). "Differential induction of cytochrome P-450 dependent monooxygenase, epoxide hydolase, glutathione transferase and UDP glucuronosyltransferase activities in the liver of rainbow trout by $B$ naphthoflavone or Clophen A50." Biochem. Pharmacol. 34(18): 3309-3314.

Armknecht, S.L., S.L. Kaattari and P.A. Van Veld (1998). "An elevated glutathione Stransferase in creosote resistant mummichog (Fundulus heteroclitus)." Aquatic Toxicol 41: 1-16.

Ballschmiter, K. and M. Zell (1980). "Analysis of polychlorinated biphenyls (PCB) by glass capillary gas chromatography." Fresnius Z. Anal. Chem. 302: 20-31.

Barton, H.A. and M.A. Marletta (1988). "Kinetic and immunochemical studies of a receptor-like protein that binds aromatic hydrocarbons." J. Biol. Chem. 263(12): 58255832.

Bernhoft, A., H. Hektoen, J.U. Skaare and K. Ingebrigtsen (1994). "Tissue distribution and effects on hepatic xenobiotic metabolising enzymes of 2,3,3',4,4'-pentachlorobiphenyl (PCB-105) in cod (Gadus morhua) and rainbow trout (Oncorhynchus mykiss)." Environ. Pollut. 85: 351-359.

Black, D.E., R. Gutjahr-Gobell, R.J. Pruell, B. Bergen, L. Mills and A.E. McElroy (1998). "Reproduction and polychlorinated biphenyls in Fundulus heteroclitus (Linnaeus) from New Bedford Harbor, Massachusetts, USA." Environ. Toxicol. Chem. 17(7): 1405-1414.

Black, D.E., D.K. Phelps and R.L. Lapan (1988). "The effect of inherited contamination on egg and larval winter flounder, Pseudopleuronectes americanus." Mar. Environ. Res. 25: $45-62$.

Bock, K.W. (1991). "Roles of UDP-Glucuronosyltransferases in Chemical Carcinogenesis." Crit. Rev. Biochem. Mol. Biol. 26(2): 129-150. 
Brown, B. and R. Chapman (1991). "Gene flow and mitochondrial DNA variation in the killifish, Fundulus heteroclitus." Evolution 45(5): 1147-1161.

Burns, K.A. (1976). "Microsomal mixed function oxidases in an estuarine fish, Fundulus heteroclitus, and their induction as a result of environmental contamination." Comp. Biochem. Physiol. 53B: 443-446.

Burt, R.K. and S.S. Thorgeirsson (1988). "Coinduction of MDR-1 multidrug-resistance and cytochrome P-450 genes in rat liver by xenobiotics." J. Natl. Cancer Inst. 80(17): 1383-1386.

Cantrell, S.M., J. Joy-Schlezinger, J.J. Stegeman, D.E. Tillitt and M. Hannink (1998). "Correlation of 2,3,7,8-tetrachlorodibenzo-p-dioxin-induced apoptotic cell death in the embryonic vasculature with embryotoxicity." Toxicol. Appl. Pharmacol. 148(1): 24-34.

Chidester, F. (1920). "The behavior of Fundulus heteroclitus on the salt marshes of New Jersey." Amer. Nat. 54: 551-557.

Clark, G., A. Tritscher, G. Lucier and M. Taylor (1991). "Dose-dependent increase in tumor necrosis factor-alpha production in TCDD exposed mice is Ah receptor dependent." Chemosphere 23(11-12): 1817-1823.

Clarke, D., B. Burchell and S. George (1992). "Differential expression and induction of UDP-glucuronosyltransferase isoforms in hepatic and extrahepatic tissues of a fish, Pleuronectes platessa: immunochemical and functional characterization." Toxicol. Appl. Pharmacol. 115: 130-136.

Collier, T.K. and U. Varanasi (1991). "Hepatic activities of xenobiotic metabolizing enzymes and biliary levels of xenobiotics in english sole (Parophrys vetulus) exposed to environmental contaminants." Arch. Environ. Contam. Toxicol. 20: 462-473.

Coombs, D.H. and N.R. Watts (1985). "Generating sucrose gradients in three minutes by tilted tube rotation." Anal. Biochem. 148(1): 254-259.

Craig, A., H. Sakamoto and C. Reinisch (1993). "Prevalence of leukemia in soft-shell clams (Mya arenaria) transplanted to New Bedford Harbor." Mar. Environ. Res. 35(1-2): 199-200.

Denison, M.S., J.M. Fisher and J.P. Whitlock (1988). "Inducible, receptor-dependent protein-DNA interactions at a dioxin-responsive transcriptional enhancer." Proc Natl Acad Sci 85: 2528-2532. 
Denison, M.S., L.M. Vella and A.B. Okey (1986b). "Structure and function of the Ah receptor for 2,3,7,8-tetrachlorodibenzo-p-dioxin: species differences in molecular properties of the receptors from mouse and rat hepatic cytosols." J. Biol. Chem. 261: 3987-3995.

DeVito, M.J., J.J. Diliberto, D.G. Ross, M.G. Menache and L.S. Birnbaum (1997). "Doreresponse relationships for polyhalogenated dioxins and dibenzofurans following subchronic treatment in mice 1. CYP1A1 and CYP1A2 enzyme activity in liver, lung, and skin." Toxicol. Appl. Pharmacol. 147: 267-280.

Dottavio-Martin, D. and J.M. Ravel (1978). "Radiolabeling of proteins by reductive alkylation with $[14 \mathrm{C}]$ formaldehyde and sodium cyanoborohydride." Analyt. Biochem. 87: 562-565.

Elskus, A., and J. Stegeman (1989). "Induced cytochrome P-450 in Fundulus heteroclitus asșociated with environmental contamination by polychlorinated biphenyls and polynuclear aromatic hydrocarbons." Mar. Environ. Res. 27: 31-50.

Elskus, A., E. Monosson, A. McElroy, J. Stegeman and D. Woltering (1999). "Altered CYP1A expression in Fundulus heteroclitus adults and larvae: a sign of pollutant resistance?" Aquat Toxicol 45: 99-113.

Elskus, A.A., R. Pruell and J.J. Stegeman (1992). "Endogenously-mediated, pretranslational suppression of cytochrome P4501A in PCB-contaminated flounder." Mar. Environ. Res. 34: 97-101.

Elskus, A.A., J.J. Stegeman, L.C. Susani, D. Black, R.J. Pruell and S.J. Fluck (1989). "Polychlorinated biphenyls concentration and cytochrome P-450E expression in winter flounder from contaminated environments." Mar. Environ. Res. 28(1-4): 25-30.

Endicott, J.A. and V. Ling (1989). "The biochemistry of P-glycoprotein-mediated multidrug resistance." A. Rev. Biochem. 58: 137-171.

Faisal, M., B.A. Weeks, W.K. Vogelbein and R.J. Huggett (1991). "Evidence of aberration of the natural cytotoxic cell activity in Fundulus heteroclitus (Pisces: Cyprinodontidae) from the Elizabeth River, Virginia." Vet. Immunol. Immunopathol. 29(3): 3-4.

Fernandez Salguero, P., D.M. Hilbert, S. Rudikoff, J.M. Ward and F.J. Gonzalez (1996). "Aryl-hydrocarbon receptor-deficient mice are resistant to 2,3,7,8-tetrachlorodibenzo-pdioxin-induced toxicity." Toxicol Appl Pharmacol 140: 173-179. 
Fernandez Salguero, P., T. Pineau, D.M. Hilbert, T. McPhail, S.S. Lee, S. Kimura, D.W. Nebert, S. Rudikoff, J.M. Ward and F.J. Gonzalez (1995). "Immune system impairment and hepatic fibrosis in mice lacking the dioxin-binding Ah receptor [see comments]." Science (United States) 268(5211): 722-6.

Fox, G.A. (1995). "Tinkering with the tinkerer: pollution versus evolution." Environ. Health Perspect. 103(4): 93-100.

Fritz, E.S., W.H. Meredith and V.A. Lotrich (1975). "Fall and winter movements and activity level of the mummichog, Fundulus heteroclitus, in a tidal creek." Chesapeake Sci. 16(3): 211-215.

Fukunaga, B.N. and O. Hankinson (1996). "Identification of a novel domain in the Aryl hydrocarbon receptor required for DNA binding." J. Biological Chemistry 271(7): 37433749 .

Gallagher, K., P. Van Veld, R. Hale and J. Stegeman (1995). "Induction of cytochrome P4501A in the mummichog (Fundulus heteroclitus) by the polychlorinated terphenyl formulation Aroclor 5432." Environ. Toxicol. Chem. 14(3): 405-409.

Giannone, J.V., W. Li, M. Probst and A.B. Okey (1998). "Prolonged depletion of AH receptor without alteration of receptor $\mathrm{mRNA}$ levels after treatment of cells in culture with 2,3,7,8-tetrachlorodibenzo-p-dioxin." Biochem Pharmacol 55: 489-497.

Gleason, T., D. Champlin, P. Edwards, K. Rocha, D. Nacci, S. Rego, J. Serbst, W. Munns, R. Gutjahr-Gobell and K. DellaVecchia (1997). Persistence of an estuarine fish population at a marine superfund site: population-level compensatory mechanisms. Society of Environmental Toxiclology and Chemistry 18th Annual Meeting, San Francisco, CA, SETAC Press.

Gooch, J.W., A.A. Elskus, P.J. Kloepper-Sams, M.E. Hahn and J.J. Stegeman (1989). "Effects of ortho- and non-ortho-substituted polychlorinated biphenyl congeners on the hepatic monooxygenase system in scup (Stenotomus chrysops)." Toxicol.Appl. Pharmacol. 98(3): 422-433.

Gradin, K., R. Toftgard, L. Poellinger and A. Berghard (1999). "Repression of dioxin signal transduction in fibroblasts. Identification of a putative repressor associated with Arnt." J Biol Chem 274(19): 13511-13518.

Gradin, K., M.L. Whitelaw, R. Toftg ard, L. Poellinger and A. Berghard (1994). "A tyrosine kinase-dependent pathway regulates ligand-dependent activation of the dioxin receptor in human keratinocytes." J Biol Chem (United States) 269(38): 23800-7. 
Habig, W.H., M.J. Pabst and W.B. Jakoby (1974). "Glutathione s-transferases.” J. Biological Chemistry 249(22): 7130-7139.

Hahn, M.E. (1998). "Mechanisms of innate and acquired resistance to dioxin-like compounds." Reviews in Toxicology. Series B - Environmental Toxicology 2: 395-443.

Hahn, M.E., S.I. Karchner, M.A. Shapiro and S.A. Perera (1997). "Molecular evolution of two vertebrate aryl hydrocarbon (dioxin) receptors (AHR1 and AHR2) and the PAS family." Proc. Natl. Acad. Sci. U.S.A. 94: 13743-13748.

Hahn, M.E., T.M. Lamb, M.E. Schultz, R.M. Smolowitz and J.J. Stegeman (1993). "Cytochrome P4501A induction and inhibition by 3,3',4,4'-tetrachlorobiphenyl in an Ah receptor-containing fish cell line (PLHC-1)." Aquat. Toxicol 26: 185-208.

Hahn, M.E., A. Poland, E. Glover and J.J. Stegeman (1994). "Photoaffinity labeling of the Ah receptor: phylogenetic survey of diverse vertebrate and invertebrate species." Arch. Biochem. Biophys. 310: 218-228.

Hahn, M.E., B.L. Woodward, J.J. Stegeman and S.W. Kennedy (1996). "Rapid assessment of induced cytochrome P4501A protein and catalytic activity in fish hepatoma cells grown in multiwell plates: Response to TCDD, TCDF, and two planar PCBs." Environ.1 Toxicol. Chem. 15(4): 582-591.

Hankinson, O. (1983). "Dominant and recessive aryl hydrocarbon hydroxylase-deficient mutants of mouse hepatoma line, Hepa-1, and assignment of recessive mutants to three complementation groups." Somatic Cell Genetics 9(4): 497-514.

Hankinson, O. (1994). "The role of the aryl hydrocarbon receptor nuclear translocator protein in aryl hydrocarbon receptor action." Trends Endocrinol Metab 5(6): 240-244.

Hankinson, O. (1995). "The aryl hydrocarbon receptor complex." Annu. Rev. Pharmacol. Toxicol. 35: 307-340.

Harper, D.M., D.A. Flessas and C.L. Reinisch (1994). "Specific reactivity of leukemia cells to polyclonal anti-PCB antibodies." J. Invertebr. Pathol. 64(3): 234-237.

Hayashi, S., K.J. Okabe, Y. Honma and K. Kawajiri (1995). "Expression of Ah receptor (TCDD receptor) during human monocytic differentiation." Carcinogenesis (England) 16(6): 1403-9.

Hayes, J.D. and D.J. Pulford (1995). "The glutathione s-transferase supergene family: regulation of GST * and the contribution of the isoenzymes to cancer chemoprotection and drug resistance." Crit Rev Biochem Mol Biol 30(6): 445-600. 
Hektoen, H., A. Bernhoft, K. Ingebrigtsen, J.U. Skaare and A. Goksoyr (1994). "Response of hepatic xenobiotic metabolizing enzymes in rainbow trout (Oncorhynchus mykiss) and cod (Gadus morhua) to 2,3,7,8-tetrachlorodibenzo-p-dioxin (2,3,7,8TCDD)." Aquatic Toxicol. 28: 97-106.

Henry, T.R., D.J. Nesbit, and R.E. Peterson (1996). "Toxic equivalency factors for polychlorinated dibenzon-p-dioxins and biphenyls in zebrafish liver cells." in Society of Environmental Toxicology and Chemistry 17th Annual Meeting. p. 270 (Abstract \#PO811)

Hitchman, N., M. Leaver and S. George (1995). "Alternatives to whole animal testing: use of CDNA probes for studies of phase I and phase II enzyme induction in isolated plaice hepatocytes." Mar. Environ. Res. 39: 289-292.

Hoffer, A., C.-y. Chang and A. Puga (1996). "Dioxin induces transcription of fos and jun genes by Ah receptor-dependent and -independent pathways." Toxicol. Appl. Pharmacol. 141(1): 238-247.

Hoffman, E.C., H. Reyes, F. Chu, F. Sander, L.H. Conley, B.A. Brooks and O. Hankinson (1991). "Cloning of a factor required for activity of the Ah (dioxin) receptor." Science 252: $954-958$.

Holsapple, M.P., N.K. Snyder, S.C. Wood and D.L. Morris (1991). “ A review of 2,3,7,8tetrachlorodibenzo-p-dioxin-induced changes in immunocompetence: 1991 update." Toxicol. 69(3): 219-255.

Hori, M., H. Kondo, N. Ariyoshi, H. Yamada, A. Hiratsuka, T. Watabe and K. Oguri (1997). "Changes in the hepatic glutathione peroxidase redox system produced by coplanar polychlorinated biphenyls in Ah-responsive and -less-responsive strains of mice: mechanism and implications for toxicity." Environ. Toxicol. Pharmacol. 3: 267-275.

Houser, W.H. and K.A. Woodfork (1990). "Evaluation of the 4S polycyclic aromatic hydrocarbon binding protein in the Harlan Sprague--Dawley rat." Carcinogenesis 11(7): 1165-1169.

Huggett, R.J., R.A. Kimerle, P.M. Mehrle, H.L. Bergman, K.L. Dickson, J.A. Fava, J.F. McCarthy, R. Parrish, P.B. Dorn, V. McFarland and G. Lahvis (1992). Introduction. Biomarkers: biochemical, physiological, and histological of antropogenic stress. R. J. Huggett, R. A. Kimerle, P. M. Mehrle and H. L. Bergman. Ann Arbor, Lewis Publishers: $1-3$. 
Huuskonen, S., P. Lindstrom-Seppa, K. Koponen and S. Roy (1996). "Effects of nonortho-substituted polychlorinated biphenyls (congeners 77 and 126) on cytochrome P4501A and conjugation activities in rainbow trout (Oncorhynchus mykiss)." Comp. Biochem. Physiol. 113C(2): 205-213.

Igarashi, T., T. Satoh, S. Ono, K. Iwashita, M. Hosokawa, K. Ueno and H. Kitagawa (1984). "Effect of steroidal sex hormones on the sex-related differences in the hepatic activities of gamma -glutamyltranspeptidase, glutathione S-transferase and glutathione peroxidase in rats." Res. Commun. Chem. Pathol. Pharmacol. 45(2): 225-232.

Karchner, S.I., W.H. Powell and M.E. Hahn (1999). "Identification and functional charcterization of two divergent aryl hydrocarbon receptors in the teleost Fundulus heteroclitus." J. Biol. Chem.: Submitted.

Kelly, E.J., C.J. Quaife, G.J. Froelick and R.D. Palmiter (1996). "Metallothionein I and II protect against zinc deficiency and zinc toxicity in mice." J Nutr 126(7): 1782-90.

Kennedy, S., S. Jones and L. Bastien (1995). "Efficient analysis of cytochrome P4501A catalytic activity, porphyrins, and total proteins in chicken embryo hepatocyte cultures with a fluorescence plate reader." Anal. Biochem. 226(2): 362-370.

Klerks, P.L. and J.D. Weis (1987). "Genetic adaptation to heavy metals in aquatic organisms: a review." Environ. Pollut. 45: 173-205.

Kloepper- Sams, P.J. and J.J. Stegeman (1989). "The temporal relationships between $\mathrm{P} 450 \mathrm{E}$ protein content, catalytic activity, and mRNA levels in the teleost Fundulus heteroclitus following treatment with beta -naphthoflavone." Arch. Biochem. Biophys. 268(2): 525-235.

Koivusaari, U., M. Harri and O. Hanninen (1981). "Seasonal variation of hepatic biotransformation in female and male rainbow trout (Salmo gairdneri)." Comp. Biochem. Physiol. 70C: 149-157.

Koponen, K., O. Ritola, S. Huuskonen, D. Linder, K. Monostory and P. Lindstrom-Seppa (1997). "Intrastrain and interstrain variability in biotransformation enzyme activities of rainbow trout (Oncorhynchus mykiss)." Can. J. Fish. Aquat. Sci. 54: 2901-2906.

Kreamer, G.L., K. Squibb, D. Gioeli, S.J. Garte and I. Wirgin (1991). "Cytochrome P450IA messenger RNA expression in feral Hudson River (New York, USA) tomcod." Environ. Res. 55(1): 64-78.

Lake, J., R. McKinney, C. Lake, F. Osterman, and J. Heltshe (1995). "Comparisons of patterns of polychlorinated biphenyl congeners in water, sediment, and indigenous 
organisms from New Bedford harbor, Massachusetts." Arch. Environ. Contam. Toxicol. 29: 207-220.

Landers, J.P. and N.J. Bunce (1991). "The Ah receptor and the mechanism of dioxin toxicity." Biochem J (England) 273(2): 273-281.

Landers, J.P., M.J. Winhall, T.L. McCready, D.A.R. Sanders, D. Rasper, J.S. Nakai and N.G. Bunce (1991). "Characterization of an inducible aryl hydrocarbon receptor-like protein in rat liver." J. Biol. Chem. 266(15): 9471-9480.

Leaver, M., D. Clarke and S. George (1992). "Molecular studies of the phase II xenobiotic conjugative enzymes of marine Pleuronectid flatfish." Aquat. Toxicol. 22: 265278.

Leaver, M. and S. George (1996). "Three repeated glutathione s-transferase genes from a marine fish, the plaice (Pleuronected platessa)." Mar. Environ. Res. 42(1-4): 19-23.

Lemaire, P., A. Mathieu, S. Carriere, J.F. Narbonne, M. Lafaurie and J. Giudicelli (1992). "Hepatic biotransformation enzymes in aquaculture European sea bass (Dicentrarchus labrax ): Kinetic parameters and induction with benzo(a)pyrene." Comp. Biochem. Physiol. 103B(4): 847-853.

Li, H., L. Dong and J.P. Whitlock (1994). "Transcriptional activation function of the mouse Ah receptor nuclear translocator." J. Biological Chemistry 269(45): 28098-28105.

Lin, F.H., G. Clark, L.S. Birnbaum, G.W. Lucier and J.A. Goldstein (1991). "Influence of the Ah locus on the effects of 2,3,7,8-tetrachlorodibenzo-p-dioxin on the hepatic epidermal growth factor." Mol. Pharmacol. 39: 307-313.

Lindebro, M.C., L. Poellinger and M.L. Whitelaw (1995). "Protein-protein interaction via PAS domains: role of the PAS domain in positive and negative regulation of the bHLH/PAS dioxin receptor-ARNT transcription factor complex." EMBO journal 14(14): 3528-3539.

Liu, J., Y. Liu, S.S. Habeebu and C.D. Klaassen (1998). "Susceptibility of MT-null mice to chronic $\mathrm{CdCl} 2$-induced nephrotoxicity indicates that renal injury is not mediated by the CdMT complex." Toxicol Sci 46(1): 197-203.

Lorenzen, A. and S.W. Kennedy (1993). "A fluorescence-based protein assay for use with a microplate reader." Anal Biochem (United States) 214(1): 346-8. 
Lorenzen, A. and A.B. Okey (1990). "Detection and characterization of $[3 \mathrm{H}] 2,3,7,8$ tetrachlorodibenzo-p-dioxin binding to Ah receptor in a rainbow trout hepatoma cell line." Toxicol. Appl. Pharmacol. 106: 53-62.

Lotrich, V. (1975). "Summer home range and movements of Fundulus heteroclitus (pisces: Cyprinodontidae) in a tidal creek." Ecology 56: 191-198.

Lusska, A., E. Shen and J.P. Whitlock (1993). "Protein-DNA interactions at a dioxinresponsive enhancer." J. Biol. Chem. 268(9): 6575-6580.

Masmoudi, T., R. Planells, J. Mounie, Y. Artur, J. Magdalou and H. Goudonnet (1996). "Opposite regulation of bilirubin and 4-nitrophenol UDP-glucuronosyltransferase mRNA levels by 3,3',5 triiodo-L-thyronine in the rat liver." FEBS Letters 379: 181-185.

Miller, M.R., D.R. Hinton and J.J. Stegeman (1989). "Cytochrome P-450E induction and localization in gill pillar (endothelial) cells of scup and rainbow trout." Aquat. Toxicol. 14: 307-322.

Mimura, J., M. Ema, K. Sogawa and Y. Fujii-Kuriyama (1999). "Identification of a novel mechanism of regulation of Ah (dioxin) receptor function." Genes Dev 13(1): 20-5.

Mimura, J., K. Yamashita, K. Nakamura, M. Morita, T.N. Takagi, K. Nakao, M. Ema, K. Sogawa, M. Yasuda, M. Katsuki and Y. Fujii-Kuriyama (1997). "Loss of teratogenic response to 2,3,7,8-tetrachlorodibenzo-p-dioxin (TCDD) in mice lacking the Ah (dioxin) receptor." Genes Cells 2(10): 645-654.

Monod, G., M.A. Boudry and C. Gillet (1996). "Biotransformation enzymes and their induction by B-naphthoflavone during embryolarval development in salmonid species." Comp. Biochem. Physiol. 114C(1): 45-50.

Monosson, E. and J.J. Stegeman (1994). "Induced cytochrome P4501A in winter flounder, Pleuronectes americanus, from offshore and coastal sites." Can. J. Fish. Aquat. Sci. 51: 933-941.

Morris, D.L. and M.P. Holsapple (1991). "Effects of 2,3,7,8-tetrachlorodibenzo-p-dioxin (TCDD) on humoral immunity: II. B cell activation." Immunopharmacology (Netherlands) 21(3): 171-81.

Morrison, H.G., E.J. Weil, S.I. Karchner, M.L. Sogin and J.J. Stegeman (1998). "Molecular cloning of CYP1A from the estuarine fish Fundulus heteroclitus and phylogenetic analysis of CYP1 genes: update with new sequences." Comp Biochem Physiol C Pharmacol Toxicol Endocrinol 121(1-3): 231-40. 
Murphy, D.L. and J.W. Gooch (1997). "EROD and CYP1A protein in channel catfish (Ictalurus punctatus) from an urban estuary relative to that in benzo[a]pyrene-exposed hatchery specimens." Environ. Pollut. 95(2): 235-239.

Nacci, D., L. Coiro, D. Champlin, S. Jayaraman, R. McKinney, T. Gleason and W.J. Munns (1999). "Adaptation of wild populations of the estuarine fish Fundulus heteroclitus to persistent environmental contaminants." Mar. Biol. 134(1): 9-18.

Nebert, D., A. Puga and V. Vasiliou (1993). "Role of the Ah receptor and the dioxininducible [Ah] gene battery in toxicity, cancer, and signal transduction." Ann N Y Acad Sci (United States) 685(624): 624-40.

Okey, A., D. Riddick, and P. Harper (1994). "The Ah receptor: mediator of the toxicity of 2,3,7,8-tetrachlorodibenzo-p-dioxin (TCDD) and related compounds." Toxicol. Letters 1994(70): 1-22.

Okey, A.B., L.M. Vella and P.A. Harper (1989). "Detection and characterization of a low affinity form of cytosolic $\mathrm{Ah}$ receptor in livers of mice nonresponsive to induction of $\mathrm{P}_{1}$ 450 by 3-methylchloanthrene." Mol. Pharmacol. 35: 823-830.

Otto, D.M. and T.W. Moon (1995). "3,3',4,4'-Tetrachlorobiphenyl effects on antioxidant enzymes and glutathione status in different tissues of rainbow trout." Pharmacol. Toxicol. 77: $281-287$.

Pangrekar, J. and H. Sikka (1992). "Xenobiotic metabolizing enzyme activity in the liver and kidney of the brown bullhead (Ictalurus nebulosus)." Mar. Environ. Res 34: 287-291.

Park, S.S., H. Miller, A.V. Klotz, P.J. Kloepper-Sams, J.J. Stegeman and H.V. Gelboin (1986). "Monoclonal antibodies to liver microsomal cytochrome P-450E of the marine fish Stenotomus chrysops (scup): cross-reactivity with 3-methylcholanthrene induced rat cytochrome P-450." Arch. Biochem. Biophys. 249: 339-350.

Peryt, B., P. Maurel and P. Lesca (1992). "Characterization of the $4 \mathrm{~S}$ polycyclic aromatic hydrocarbon-binding protein in human liver and cells." Arch. Biochem. Biophys. 298(2): 420-430.

Peterson, T.C., F.J. Gonzalez and D.W. Nebert (1986). "Methylation differences in the murine $P_{1} 450$ and $P_{3} 450$ genes in wild-type and mutant hepatoma cell culture." Biochem. Pharmacol. 35(13): 2107-2114.

Pohjanvirta, R., J. Wong, L. Wei, P.A. Harper, J. Tuomisto and A.B. Okey (1998). "Point mutation in intron sequence causes altered carboxyl-terminal structure in the aryl 
hydrocarbon receptor of the the most TCDD-resistant rat strain." Mol. Pharmacol. 54: 8693.

Poland, A., D. Palen, and E. Glover (1994). "Analysis of the four alleles of the murine aryl hydrocarbon receptor." Mol. Pharmacol. 46: 915-921.

Poland, A. and J.C. Knutson (1982). "2,3,7,8-tetrachlorodibenzo-p-dioxin and related halogenated aromatic hydrocarbons: examination of the mechanism of toxicity." Ann Rev Pharmacol Toxicol 22: 517-554.

Pollenz, R.S. (1996). "The aryl hydrocarbon receptor, but not the aryl hydrocarbon receptor nuclear translocator protein, is rapidly depleted in hepatic and nonhepatic culture cells exposed to 2,3,7,8-tetrachlorodibenzo-p-dioxin." Mol. Pharmacol. 49: 391-398.

Pollenz, R.S. and B. Necela (1998). "Characterization of two continuous cell lines derived from Oncorhynchus mykiss for models of aryl-hydrocarbon-receptor-mediated signal transduction. Direct comparison to the mammalian Hepa-iclc7 cell line." Aquat. Toxicol. 41(1-2): 31-49.

Pongratz, I., C. Antonsson, M.L. Whitelaw and L. Poellinger (1998). "Role of the PAS domain in regulation of dimerization and DNA binding specificity of the dioxin receptor." Mol Cell Biol 18(7): 4079-88.

Powell, W.H., S.I. Karchner, R. Bright and M.E. Hahn (1999). "Functional diversity of vertebrate ARNT proteins: identification of ARNT2 as the predominate form of ARNT in the marine teleost, Fundulus heteroclitus." Arch Biochem Biophys 361(1): 156-163.

Powers, D.A., I. Ropson, D.C. Brown, B.R. Van, R. Cashon, V.L. Gonzalez and J.A. DiMichele (1986). "Genetic variation in Fundulus heteroclitus : Geographic distribution." Am. Zool. 26(1): 131-141.

Prince, R., and K. Cooper (1995a). "Comparisons of the effects of 2,3,7,8tetrachlorodibenzo- $p$-dioxin on chemically impacted and nonimpacted subpopulations of Fundulus heteroclitus: I TCDD toxicity." Environ. Toxicol. Chem. 14(4): 579-587.

Prince, R., and K. Cooper (1995b). "Comparisons of the effects of 2,3,7,8tetrachlorodibenzo- $p$-dioxin on chemically impacted and nonimpacted subpopulations of Fundulus heteroclitus: II metabolic considerations." Environ. Toxicol. Chem. 14(4): 589595.

Pruell, R.J., C.B. Norwood, R.D. Bowen, W.S. Boothman, P.F. Rogerson, M. Hackett and B.C. Butterworth (1990). "Geochemical study of sediment contamination in New Bedford Harbor, Massachusetts." Mar. Environ. Res. 29(2): 77-101. 
Raha, A., V. Reddy, W. Houser and E. Bresnick (1990). "Binding characteristics of 4S PAH-binding protein and Ah receptor from rats and mice." J. Toxicol. Environ. Health 29(4): 339-355.

Reick, M., R.W. Robertson, D.S. Pasco and J.B. Fagan (1994). "Down-regulation of nuclear aryl hydrocarbon receptor DNA-binding and transactivation functions: requirement for a labile or inducible factor." Mol Cell Biol (United States) 14(9): 56535660.

Roesijadi, G. (1992). "Metallothioneins in metal regulation and toxicity in aquatic animals." Aquat. Toxicol. 22(2): 81-114.

Roman, B.L., R.S. Pollenz and R.E. Peterson (1998). "Responsiveness of the adult male rat reproductive tract to 2,3,7,8-tetrachlorodibenzo-p-dioxin exposure: Ah receptor and ARNT expression, CYP1A1 induction, and Ah receptor down-regulation." Toxicol. Appl. Pharmacol. 150(2): 228-239.

Roy, N.K. and I. Wirgin (1997). "Characterization of the aromatic hydrocarbon receptor gene and its exrpession in Atlantic tomcod." Arch. Biochem. Biophys. 344(2): 373-386.

Safe, S. (1993). “Toxicology, structure-function relationship, and human and environmental health impacts of polychlorinated biphenyls: progress and problems." Environ Health Perspect 100: 259-268.

Safe, S. and V. Krishnan (1995). "Cellular and molecular biology of aryl hydrocarbon (Ah) receptor-mediated gene expression." Arch Toxicol Suppl (Germany) 17(99): 99115 .

Satoh, M., N. Nishimura, Y. Kanayama, A. Naganuma, T. Suzuki and C. Tohyama (1997). "Enhanced renal toxicity by inorganic mercury in metallothionein-null mice." J Pharmacol Exp Ther 283(3): 1529-33.

Schlezinger, J.J. and J.J. Stegeman (1999). "Oxidative inactivation of cytochrome P4501A and production of reactive oxygen stimulated by halogenated aryl hydrocarbon receptor agonists." Mol. Pharmacol.: In Press.

Schlezinger, J.J. and J.J. Stegeman (1999). "Tissue distribution of and induction of cytochrome P4501A by model aryl hydrocarbon receptor (AhR) agonists in the American eel." Aquat. Toxicol.: Submitted.

Schuetz, E.G., J.D. Schuetz, M.T. Thompson, R.A. Fisher, J.R. Madariage and S.C. Strom (1995). "Phenotypic variability in induction of P-glycoprotein mRNA by aromatic hydrocarbons in primary human hepatocytes." Mol. Carcinog. 12(2): 61-65. 
Sikoki, F., R. Tubb and L. Curtis (1989). "Elevation of sex-steroids and inhibition of udpglucuronyltransferase are out of phase during gonadal maturation in the common carp." Comp. Biochem. Physiol. 92C: 267-272.

Smolowitz, R.M., M.E. Hahn and J.J. Stegeman (1991). "Immunohistochemical localization of cytochrome P4501A1 induced by 3,3',4,4'-tetrachlorobiphenyl and by 2,3,7,8-tetrachlorodibenzofuran in liver and extrahepatic tissues of the teleost Stenotomus chrysops (scup)." Drug. Metab. Dispos. 19: 113-123.

Stegeman, J., R. Binder and A. Orren (1979). "Hepatic and extrahepatic microsomal electron transport components and mixed-function oxygenases in the marine fish Stenotomus versicolor." Biochem. Pharmacol. 28: 3431-3439.

Stegeman, J., R. Smolowitz and M. Hahn (1991). "Immunohistochemical localization of environmentally induced cytochrome P4501A1 in multiple organs of the marine teleost Stenotomus chrysops (Scup)." Toxicol. Appl. Pharmacol. 110: 486-504.

Stegeman, J.J., M. Brouwer, R.T. Di Giulio, L. Forlin, B.A. Fowler, B.M. Sanders and P.A. Van Veld (1992). Molecular responses to environmental contamination:enzyme and protein systems as indicators of chemical exposure and effect. Biomarkers: biochemical, physiological, and histological of antropogenic stress. R. J. Huggett, R. A. Kimerle, P. M. Mehrle and H. L. Bergman. Ann Arbor, Lewis Publishers: 235-335.

Stegeman, J.J. and M.E. Hahn (1994). Biochemistry and molecular biology of monooxygenases: current perspectives on forms functions, and regulation of cytochrome P450 in aquatic species. Aquatic toxicology. D. C. Malins and G. K. Ostrander. Ann Arbor, Lewis publishers: 87-206.

Sutter, T., and W. Greenlee (1992). "Classification of members of the Ah gene battery." Chemosphere 25(1-2): 223-226.

Swanson, H.I. and C.A. Bradfield (1993). "The Ah-receptor: genetics, structure and function." Pharmacogenetics 3: 213-230.

Taylor, M.H. (1986). "Environmental and endocrine influences on reproduction of Fundulus heteroclitus." Am. Zool. 26(1): 159-171.

Taysse, L., C. Chambras, D. Marionnet, C. Bosgiraud and P. Deschaux (1998). "Basal level and induction of Cytochrome P450, EROD, UDPGT, and GST activities in carp (Cyprinus carpio) immune organs (spleen and head kidney)." Bull. Environ Contam. Toxicol 60: 300-305. 
Tsui, H.W. and A.B. Okey (1981). "Rapid vertical tube rotor gradient assay for binding of 2,3,7,8-tetrachlorodibenzo-p-dioxin to the Ah receptor." Can. J. Physiol. Pharmacol. 59: 927-931.

Van Veld, P., W. Vogelbein, M. Cochran, A. Goksoyr and J. Stegeman (1997). "Routespecific cellular expression of cytochrome P4501a (CYP1A) in fish (Fundulus heteroclitus) following exposure to aqueous and dietary benzo(a)pyrene." Toxicol. Appl. Pharmacol. 142: 348-359.

Van Veld, P.A., U. Ko, W.K. Vogelbein and D.J. Westbrook (1991). "Glutathione Stransferase in intestine, liver and hepatic lesions of mummichog (Fundulus heteroclitus ) from a creosote-contaminated environment." Fish Physiol. Biochem. 9(4): 369-376.

Van Veld, P.A., W.K. Vogelbein, R. Smolowitz, B. Woodin and J.J. Stegeman (1992). "Cytochrome P4501A1 in hepatic lesions of a teleost fish (Fundulus heteroclitus) collected from a polycyclic aromatic hydrocarbon-contaminated site." Carcinogenesis 13(3): 505-507.

Van Veld, P.A. and D.J. Westbrook (1995). "Evidence for depression of cytochrome P4501A in a population of chemically resistant mummichog (Fundulus heteroclitus)." Environ Sci 3(4): 221-234.

Vasiliou, V., H.G. Shertzer, R.M. Liu, M. Sainsbury and D.W. Nebert (1995). "Response of [Ah] battery genes to compounds that protect against menadione toxicity." Biochem Pharmacol (England) 50(11): 1885-91.

Vodicnik, M.J., C.R. Elcombe and J.J. Lech (1981). "The effect of various types of inducing agents on hepatic microsomal monooxygenase activity in rainbow trout." Toxicol. Appl. Pharmacol. 59(364).

Vogelbein, W.K., J.W. Fournie, P. Veld and R.J. Huggett (1990). "Hepatic neoplasms in the mummichog Fundulus heteroclitus from a creosote-contaminated site." Cancer Res. 50(18): 5978-5986.

Washburn, B., C. Vines, D. Baden, D. Hinton and P. Walsh (1996). "Differential effects of brevetoxin and B-naphthoflavone on xenobiotic metabolizing enzymes in striped bass (Morone scaxatilis)." Aquat. Toxicol. 35: 1-10.

Watson, A.J., B.K. Weir, R.M. Bannister, F.F. Chu, P.S. Reisz, K.Y. Fujii, K. Sogawa and O. Hankinson (1992). "Mechanism of action of a repressor of dioxin-dependent induction of Cyplal gene transcription." Mol Cell Biol (United States) 12(5): 2115-23. 
Weaver, G. (1984). "PCB Contamination in and around New Bedford, Mass." Environ. Sci. Technol. 18: 22A-27A.

Weis, J.S. and P. Weis (1989). "Tolerance and stress in a polluted environment." Bioscience 39(2): 89-95.

Weis, J.S., P. Weis, M. Herber and S. Vaidya (1981). "Methylmercury Tolerance of Killifish (Fundulus heteroclitus ) Embryos From a Polluted vs Non-Polluted Environment." Mar. Biol. 65(3): 283-287.

Weis, P. and J.S. Weis (1982). "Toxicity of the PCBs Aroclor 1254 and 1242 to Embryos and Larvae of the Mummichog, Fundulus heteroclitus." Bull. Environ. Contam. Toxicol. 28(3): 298-304.

White, R.D., D. Shea, A.R. Solow and J.J. Stegeman (1997). "Induction and posttranscriptional suppression of hepatic cytochrome P450 1A1 by 3,3',4,4'tetrachlorobiphenyl." Biochem Pharmacol 53(7): 1029-40.

Whitelaw, M., I. Pongratz, A. Wilhelmsson, J.A. Gustafsson and L. Poellinger (1993). "Ligand-dependent recruitment of the Arnt coregulator determines DNA recognition by the dioxin receptor." Mol Cell Biol (United States) 13(4): 2504-14.

Whitlock, J.P. (1990). "Genetic and molecular aspects of 2,3,7,8-tetrachlorodibenzo-pdioxin action." Annu Rev Pharmacol Toxicol 30: 251-277.

Willett, K., M. Steinberg, J. Thomsen, T.R. Narasimhan, S. Safe, S. McDonald, K. Beatty and M.C. Kennicutt (1995). "Exposure of killifish to benzo[a]pyrene: Comparative metabolism, DNA adduct formation and aryl hydrocarbon (Ah) receptor agonist activities." Comp. Biochem.Physiol. 112B(1): 93-103.

Wilson, C.L., J. Thomsen, D.J. Hoivik, M.T. Wormke, L. Stanker, C. Holtzapple and S.H. Safe (1997). "Aryl hydrocarbon (Ah) nonresponsiveness in estrogen receptor-negative MDA-MB-231 cells is associated with expression of a variant ARNT protein." Arch. Biochem. Biophys. 346(1): 65-73.

Wirgin, II, G.L. Kreamer, C. Grunwald, K. Squibb and S.J. Garte (1992). "Effects of prior exposure history on cytochrome P4501A mRNA induction by PCB congener 77 in Atlantic tomcod." Mar. Environ. Res. 34(1-4): 103-108.

Wu, L. and J.P. Whitlock (1993). "Mechanism of dioxin action: receptor-enhancer interactions in intact cells." Nucleic Acids Res. 21(1): 119-125. 
Yull-Rhee, G., B. Bush, C. Bethoney, A. DeNucci, H. Oh and R. Sokol (1993). "Reductive dechlorination of AROCLOR 1242 in anaerobic sediments: pattern, rate and concentration dependence." Environ Toxicol Chem 12: 1025-1032.

Zabel, E.W., R. Pollenz and R.E. Peterson (1996). "Relative potencies of individual polychlorinated dibenzo-p-dioxin, dibenzofuran, and biphenyl congeners and congener mixtures based on induction of cytochrome P4501A mRNA, in a rainbow trout gonadal cell line (RTG-2)." Environ.1 Toxicol. Chem. 15(12): 2310-2318. 